This dissertation has been

$63-2559$

microfilmed exactly as received

STEELE, Lawrence Russell, 1935-

WATER DECOMPOSITION BY FISSION FRAGMENT RECOIL ENERGY IN AN AQUEOUS SLURRY OF URANIUM-THORIUM OXIDES.

The Ohio State University, Ph.D., 1962

Engineering, chemical

University Microfilms, Inc., Ann Arbor, Michigan 


\title{
WATER DECOMPOSITION BY FISSION FRAGMENT \\ RECOIL ENERGY IN AN AQUEOUS SLURRY \\ OF URANIUM-THORIUM OXIDES
}

\begin{abstract}
DISSERTATION
Presented in Partial Fulfillment of the Requirements for the Degree Doctor of Philosophy in the Graduate School of the Ohio State University
\end{abstract}

By

Lawrence Russell Steele, B.Ch.E., M.Sc.

\author{
$* * * * * *$ \\ The OhIo State University \\ 1962
}

Approved by

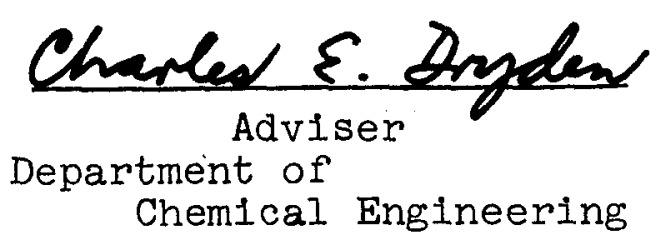




\section{ACKNOWLEDGMENTS}

For their many helpful suggestions and comments, the author is very grateful to his adviser, Dr. C. E. Dryden, and to his supervisers at Argonne, Dr. S. Gordon and Dr. E. J. Hart. Many other personnel of Argonne National Laboratory have made this thesis possible. In particular, the author wishes to acknowledge $J$. Nixon and the Central Shops Department for their help in the design and fabrication of the in-pile irradiation equipment, Dr. C. E. Crouthamel and his group for supplying the solid fuel particles, the Reactor Operations Division for their help with the irradiations, Dr. J. Hines and his group for doing some of the analytical work, and D. Carson for doing the programming for the computer. 
I. SUMMARY I

II. INTRODUCTION 3

III. LITERATURE SURVEY 6

A. The radiation chemistry of water 6

$\begin{array}{ll}\text { B. Water decomposition in nuclear } & 6 \\ \text { reactors } & 9\end{array}$

C. The range-energy relationship for 11 fission recoll fragments

IV. THEORY

A. Introduction

B. Range-energy relationship

C. Range of the fission fragments

D. Physical properties of the slurry

E. Mechanics of the calculation

F. Results of the calculation

V. EXPERIMENTAL PROGRAM

A. General approach

B. Reactor facilities

C. Reactor radiation vessel

D. Gas collection equipment

E. Gamma irradiation facility

F. Sample preparation

G. Particle size measurement

H. Gas analysis

I. Energy measurement

J. Design of experiment

K. Typlcal run

VI. RESULTS
A. Primary
B. Gas analyses
C. Gas flow rate through the slurry
D. Total fission energy
E. Effective fission energy

VII. DISCUSSION OF RESULTS

A. Hydrogen yield

B. Analysis of variance

C. Effect of stripping

D. Energy transfer

E. Dose rate

F. Alternate models

G. Fraction of particles escaping

H. Comparison with other data 
VIII. CONCLUSIONS

$\begin{array}{ll}\text { IX. RECOMMENDATIONS } & 107\end{array}$

X. BIBLIOGRAPHY 109

XI. NOMENCLATURE $\quad 114$

APPENDIX I - Data 117

Experimental Conditions 118

$\begin{array}{ll}\text { Observations } & 118 \\ \text { Gas analyses } & 118\end{array}$

Fission product analysis 138

Neutron flux measurements $\quad 146$

Particle size analyses 149

APPENDIX II - Derivations 163

Range energy relationship $\quad 164$

APPENDIX III - Sample Calculations 165

Range 166

Range-energy relationship 166

Gas analysis 167

Fission product analysis $\quad 167$

Cr51 monitor $\quad 167$

Gas flow rate 170

Energy determination $\quad 170$

Particle size 171

Fission product monitor 172

APPENDIX IV - Computer Program 173

AUTOBIOGRAPHY 183 
TABLES

TABLE PAGE

1 RANGES OF FISSION FRAGMENTS 18

2 ANALYSIS OF VARIANCE IN GAS SAMPLING 72

3 GAS FLOW RATE 73

4. ANALYSIS OF VARIANCE FOR HYDROGEN RATE 75

5 TOTAL RATE OF FISSION ENERGY IN THE SLURRY 78

6 PARTICLE SIZE ANALYSES - RESULTS 80

7 FRACTION OF FISSION ENERGY RELEASED 82

8 HYDROGEN YIELDS . $\quad 85$

9 ANALYSIS OF VARIANCE - EFFECTS OF PARTICLE 87

10 ANAIYSIS OF VARIANCE - EFFECT OF ENERGY 87

11 EXPERIMENTAL CONDITIONS 119

$\begin{array}{ll}12 & 120\end{array}$

13 GAS ANALYSES 139

14 FISSION PRODUCT ANALYSES 145

15 NEUTRON FLUX MONITOR 147

16 PARTICLE SIZE ANALYSES - PARAMETERS 150

17 PARTICLE SIZE ANALYSES - SEDIMENTATION RATE 151

18 SAMPLE OF DATA - MONTE CARLO CALCULATION 181 OF ENERGY LOST IN $\mathrm{UO}_{2}-\mathrm{ThO}_{2}$ SLURRY 


\section{FIGURES}

FIGURE

PAGE

1 EFFECT OF PARTICLE SIZE AND CONCENTRATION ON ENERGY EFFICIENCY

22

2 EFFECT OF RANGE RATIO $R_{f} / R_{S}$ ON THE

23

3 FRACTION OF FRAGMENTS STOPPED IN SOLID

25

4 EFFECT OF THE RANGE RATIO ON THE FRACTION OF FRAGMENTS STOPPED IN SOLID

26

5 ENERGY SPECTRUM OF THE FRAGMENTS LEAVING THE PARENT SPHERE

28

6 EFFECT OF RANGE-ENERGY MODEL ON ENERGY EFFICIENCY

29

7 DIAGRAM OF THE CP-5 REACTOR FACILITIES 34

8 CP-5 THERMAL COLUMN BEAM CATCHER 35

9 REACTOR IRRADIATION VESSEL 37

10 SLURRY VESSEL AND GEAR BOX ASSEMBLIES 38

11 PHOTOGRAPH OF THE REACTOR IRRADIATION 43

12 PHOTOGRAPH SHOWING THE POSITION OF THE VESSEL WHEN. WITHDRAWN IN TO THE BEAM CATCHER

13 FLOW DIAGRAM OF GAS COLLECTION EQUIPMENT 46

14 GAS SAMPLING APPARATUS 50

15 ARRANGEMENT OF APPARATUS USED WITH 51 GAMMA SOURCE

16 PHOTOMICROGRAPH OF URANIA-THORIA PARTICLES 54

17 DIAGRAM OF THE SEDIMENTATION APPARATUS . 56

18 PHOTOGRAPHS SHOWING POSITION OF EQUIPMENT 64 PRIOR TO THE START OF AN IRRADIATION

19 HYDROGEN YIELD WITH EXPERIMENTAL ERROR 86 


\section{FIGURES (contd.)}

FIGURE

PAGE

20 HYDROGEN YIELD 90

21 HYDROGEN YIELD UNDER GAMMA IRRADIATION 93

22 EFFECT OF SOIIDS ON THE OXIDATION OF 94 $\mathrm{Fe}-\mathrm{Cu}$ DOSIMETRY SOLUTION

23 ALTERNATE MODELS OF RANGE-ENERGY 96 RELATIONSHIPS

24 FISSION PRODUCTS IN THE SOLID 99

25 EFFECT OF $\mathrm{pH}$ ON HYDROGEN YIELDS 102

26 HYDROGEN FORMATION FOR VARIOUS L.E.T. 103

27 HYDROGEN ANALYSIS CALIBRATION 144

28 GAMMA ENERGY SPECTRUM FROM CHROMIUM 148

29 HYDROGEN AND OXYGEN PEAKS FROM THE GAS 168 CHROMATOGRAPH

30 CALIBRATION CURVE FOR FISSION PRODUCTS 169

31 VOLUME OF SLURRY CONSIDERED IN THE
COMPUTER PROGRAM 


\section{SUMMARY}

The radiolytic gases, hydrogen and oxygen, are products of the interaction of ionizing radiation with water in a nuclear reactor. The rate at which the water decomposes depends upon the type of radiation, as well as its intensity. Fission recoil fragments, to which most of the energy of the fission process is released, produce more radiolytic gas than any other type. As a consequence, water decomposition is a much more serious problem in reactors with fuel systems that allow the fission recoils to escape to the water.

In a slurry fuel system some of the fission recoil energy is absorbed by the solid, the other by the liquid. Calculations of this fraction as a function of both particle size and concentration of the slurry have been made with the aid of a high-speed digital computor. In the model used, it was assumed that the distance a fission recoll may travel in a particular medium is proportional to the two-thirds power of its energy.

An experimental program was planned so that the rate of decomposition of water in a slurry fuel system could be measured. During its irradiation in the CP-5 reactor thermal column, the slurry was kept in suspension by a mechanical stirrer. By passing nitrogen through the slurry during the irradiation, the radiolytic gases were stripped from the slurry before they could recombine. Average 
particle size in the slurries was varied from 6 to 50 microns in diameter. Concentration of the solid, which contained 10 per cent natural uranlum, was varled from 300 to 1,000 grams of solids per liter.

To be able to correlate the experimental data, the fraction of fission recoll energy that had escaped to the water had to be known. It could not be measured experimentally, although the total fission energy was measurable. This informationtion was thus obtained from the computer calculations. The experimental results are reported in terms of $G\left(\mathrm{H}_{2}\right)_{f}$, the number of molecules of hydrogen measured for every $100 \mathrm{ev}$. of fission fragment kinetic energy absorbed in the water. The $G\left(H_{2}\right)_{f}$ value was found to be $2.1 \pm 0.2$. Although this value is higher than that previously reported for the yield in solutions or uranium, it is still in good agreement with expected values.

The relative number of volatile fission products that escaped the solid during an irradiation were also measured. These data agree with the values predicted from the computer calculations and, therefore, support the validity of the computer program.

The results of this work may be used to help predict the amount of water decomposition to be expected in a slurry reactor. Perhaps what is more important is that an experimental technique for studying the effects of fission recoils has been demonstrated. 


\section{INTRODUCTION}

In the fission process approximately 85 per cent of the energy is released as the kinetic energy of the two fragments of the uranlum nucleus. The rest of the energy is in the form of $\gamma$-rays, $\beta$-rays, and kinetic energy of the neutrons. Although most of the kinetic energy of these particles is dissipated throughout the medium in the form of heat, some chemical effects are caused by the interaction of these highly energetic particles with surrounding molecules.

The slowing down of uncharged particles generally results in the production of charged particles. The $\gamma$-rays produce electrons by photoelectric effect, Comptom scattering, and pair production. Neutrons produce protons or larger charged particles by recoil reactions and $\gamma$-rays by scattering and capture reactions.

The kinetic energy of charged particles is absorbed along the track of the particle in the form of excitation and ionization of neighboring molecules. In a water medium the excited and ionized molecules produce hydrogen and hydroxyl free radicals. The number of free radicals initially formed depends on the energy absorbed. The rate at which the energy is absorbed, defined as either the stopping power, $\mathrm{dE} / \mathrm{dx}$, or 1 ts equivalent, Ilnear energy transfer, LET, determines the initial distribution of the free radicals. 
Because of their initial spatial distribution, there is some probability that a free radical w111 react with another before 1 t has time to diffuse away from the track of the lonizing particle. Molecules of water, hydrogen, and hydrogen peroxide are formed in such reactions. Since the distribution of free radicals depends upon the LET of the particle, the probability of recombination in the track increases with the LET. For fission fragments, which have a very high LET, the probability of recombination is close to unity.

In a water-moderated nuclear reactor the actual decomposition of the water due to the slowing down of these particles depends upon the ratio of the number of free radicals which combine in the track to the number which diffuse away from the track. Thls is because the free radicals are able to recombine the molecules of hydrogen and hydrogen peroxide to form water. The extent to which this recombination takes place in the reactor depends upon the relative concentrations of free radicals and molecules. Addition of hydrogen to the water from an external source promotes the recombination. Removing the hydrogen as it is formed by bolling the water prevents recombination.

Water decomposition in such reactors is a problem for several reasons. The presence of oxygen, product of hydrogen peroxide decomposition, promotes corrosion. Accumulation of hydrogen and oxygen as noneondensible gases may 
result in an explosion. The cost of replacing the water lost in a heavy-water reactor could make the system economically undesirable.

In homogeneous reactors the problem is much more serious than it is in reactors of the heterogeneous type. The thin cladding around the fuel of a heterogeneous reactor prevents the fission fragments from entering the water. In a solution-fueled homogeneous reactor all of the fission fragments enter the water. In a homogeneous slurry system ( solid fuel particles suspended uniformly in the water) some of the fission fragment kinetic energy will be absorbed in the solid phase. In the absence of recombination of the radiolytic gases, hydrogen and oxygen, the rate of decomposition should be a maximum for the solution fuel and a minimum for the clad fuel. That for the slurry fuel should be somewhere between the two. The problem undertaken here is to measure experimently and to calculate from theory the rate of formation of the radiolytic gases due to the kinetic energy of the fission fragments in a slurry reactor system. This rate is expected to be a function of both the particle size and the concentration of solids in the water.

The results of this investigation should provide a basis to be used in calculating the rate of water decomposition expected from any slurry reactor. They should also indicate the fraction of fission fragment energy deposited in the fluid for any fluid-solid system. 


\section{LITERATURE SURVEY}

The radiation chemistry of water

Much work has been done to try to learn the behavior of water in the presence of lonizing radiation. This work has been summarized recently by Hart and Platzman (1) and Allen (2).

In general, when any highly energetic charged particle passes through water, it excites and ionizes some of the molecules along its track. The reactions describing this process are

$$
\begin{aligned}
\mathrm{H}_{2} \mathrm{O}+\text { energy } & =\mathrm{H}_{2} \mathrm{O}^{+}+\mathrm{e}^{-} \\
\mathrm{H}_{2} \mathrm{O}^{+}+\mathrm{H}_{2} \mathrm{O} & =\mathrm{OH}+\mathrm{H}_{3} \mathrm{O}^{+} \\
\mathrm{H}_{2} \mathrm{O}+\mathrm{e}^{-} & =\mathrm{H}+\mathrm{OH}^{-} \\
\mathrm{H}_{2} \mathrm{O}+\mathrm{e}^{-} & =\mathrm{H}_{2} \mathrm{O}^{*}+\mathrm{e}^{-} \\
\mathrm{H}_{2} \mathrm{O}^{*} & =\mathrm{H}+\mathrm{OH}
\end{aligned}
$$

The $G(X)$ is defined as the number of molecules of $X$ measured for every $100 \mathrm{ev}$. absorbed. A lower case letter "g" as $g(X)$ refers to the number of molecules of $X$ formed in the track that subsequently escape the track of the ionizing particle. For the above process $\mathrm{g}\left(-\mathrm{H}_{2} \mathrm{O}\right)$ total, the number of molecules decomposed, has been estimated to be about 4.7 for all types of radiation (3). Because of the recombination effect discussed in the next paragraph, the value for $g\left(-\mathrm{H}_{2} \mathrm{O}\right)$ is always somewhat less than 4.7 . 
Since these reactions take place along the track of the particle, the initial distribution of the free radicals $\mathrm{H}$ and $\mathrm{OH}$ depends on the rate of energy transfer of the particle, i.e., LET. Before the free radicals have time to diffuse away from the track of the particle, the following reactions may take place:

$$
\begin{aligned}
\mathrm{H}+\mathrm{H} & =\mathrm{H}_{2} \\
\mathrm{OH}+\mathrm{OH} & =\mathrm{H}_{2} \mathrm{O}_{2} \\
\mathrm{H}+\mathrm{OH} & =\mathrm{H}_{2} \mathrm{O}
\end{aligned}
$$

The extent to which these reactions consume the free radicals depends upon their initial distribution and thus on the LET. For Co ${ }^{60} \gamma$-rays which have an LET equal to 0.02 ev/ $\AA, g\left(\mathrm{H}_{2}\right)$ in acid is about 0.45 (4). Boyle, et.al, (5) have found $g\left(\mathrm{H}_{2}\right)$ to be about 1.8 for fission fragments, for which the LET averages $400 \mathrm{ev} / \AA$. If all the hydrogen atoms formed in the track combined to form hydrogen molecules, $g\left(\mathrm{H}_{2}\right)$ would be half of 4.7 . The fate of the free radicals after they escape from the track depends upon the purity of the water. In the absence of impurities, the free radicals are able to react with the molecular products by the following reactions:

$$
\begin{aligned}
\mathrm{H}+\mathrm{H}_{2} \mathrm{O}_{2} & =\mathrm{H}_{2} \mathrm{O}+\mathrm{OH} \\
\mathrm{OH}+\mathrm{H}_{2} & =\mathrm{H}_{2} \mathrm{O}+\mathrm{H}
\end{aligned}
$$

The rates of recombination depend then on the relative concentrations of both the free radicals and the molecular products. Since the pair of recombination reactions 
is a chain reaction, increasing the concentration of molecular hydrogen increases the length of the chain for there is less competition with chaln terminating reactions. Net decomposition of the water can therefore be prevented by increasing the concentration of hydrogen when the gross rate of formation of molecular products is greater than that of free radicals (6). By the same reasoning, removal of the hydrogen by bolling or bubbling with an inert gas prevents recombination. The net reaction of the free radicals on the hydrogen peroxide left in the water is decomposition to water and oxygen (7).

$$
\begin{aligned}
& \mathrm{OH}+\mathrm{H}_{2} \mathrm{O}_{2}=\mathrm{HO}_{2}+\mathrm{H}_{2} \mathrm{O} \\
& \mathrm{HO}_{2}+\mathrm{HO}_{2}=\mathrm{H}_{2} \mathrm{O}_{2}+\mathrm{O}_{2} \\
& \mathrm{HO}_{2}+\mathrm{OH}=\mathrm{H}_{2} \mathrm{O}+\mathrm{O}_{2}
\end{aligned}
$$

The presence of impurities in the water may hinder the recombination reaction. Since the free radicals are strong oxidizing and reducing agents, they may react with the impurities. The reaction of lodide ion is an example:

$$
\begin{aligned}
\mathrm{OH}+\mathrm{I}^{-} & =\mathrm{OH}^{-}+\mathrm{I} \\
\mathrm{I}+\mathrm{I} & =\mathrm{I}_{2} \\
\mathrm{H}+\mathrm{I}_{2} & =\mathrm{H}^{+}+\mathrm{I}^{-}+\mathrm{I}
\end{aligned}
$$

A concentration of $10^{-4} \mathrm{M}$ lodide $10 \mathrm{n}$ is sufficient to prevent any noticeable recombination even under high intensity irradiation $(4,8,9)$. Impurities of such concentrations have no effect on the reactions which take place in or near the track of the particle. 
Temperature of the water has no effect on the rate of decomposition. However, the rates of recombination, which takes place throughout the medium, do increase with temperature (10).

The effect of $\mathrm{pH}$ on the yleld of molecular hydrogen has not been well defined. On the yleld due to $\gamma$-rays, Allen (4) belleves there is no effect of $\mathrm{pH}$, whereas Hart (3) has found a decrease in $\mathrm{g}\left(\mathrm{H}_{2}\right)$ below a $\mathrm{pH}$ of 3 . In their work with fission recolis, Boyle et al. (5) found an apparent decrease of $\mathrm{g}\left(\mathrm{H}_{2}\right)$ with $\mathrm{pH}$. Water decomposition in nuclear reactors

In water-cooled heterogeneous reactors some type of cladding separates the fissionable fuel from the water. Since the distance that the fission recoll particles travel is very short--less than 15 microns in aluminum (I1), none of their kinetic energy escapes to the water. The effect of radiation on the water is therefore due to the gamma and fast neutron flux. Under these conditions water decomposition should not be a problem because there is an excess of free radicals for promoting the recombination reaction.

In practice, much higher rates of decomposition are found in bolling reactors than in the nonbolling types. Less than one atmosphere excess pressure of hydrogen was sufficient to prevent any water decomposition in the 
Shlppingport pressurlzed water reactor (12). Heavy water decomposition in the $\mathrm{NRX}$ reactor was less than a liter per hour when operating at 26 megawatts (13) In contrast, hydrogen evolution rates in the EBWR indicated that only about half of the inftially decomposed water was recombined in the Ilquid (14). This high rate of decomposition of water in the EBWR was probably due to the low concentration of hydrogen in the bolling water.

Decomposition in aqueous homogeneous reactors is much more of a problem because the fission fragments may enter the water. Since very few free radicals escape the track of a fission recoll, the effect of the fission recolls in the water is to increase the rate of decomposition without increasing the rate of recombination.

In solution-type reactors the rate of decomposition should be a maximum. Here, all of the kinetic energy of the fission recoll fragments--about $85 \%$ of the energy from the fission process (15)--1s absorbed by the solution. The ratio of free radicals to molecular products is so low that thousands of pounds per square inch of hydrogen pressure would be needed to prevent any net decomposition of water (16). Since such a high pressure is not practical, it was necessary to find some other means of catalyzing the recombination. Cupric ions were found to catalyze the recombination of hydrogen and oxygen in the solution (17). That 
wh1ch could not be handled by the copper was passed through an external catalytic recombiner (18).

The slurry-type reactor is defined as homogeneous, but 1t actually consists of solid fuel particles suspended in a fluid. Reactors of this type were first considered in 1940 (19), and a number of slurry reactors have been proposed since then (19-24). Over the period of years, a number of the problems of handling the slurry core have been solved, but an operating slurry reactor has yet to be built.

The problem of water decomposition in slurry reactors is of the same order of magnitude as that in solution systems. At Oak Ridge National Laboratory, measurements of the water decomposition in a slurry system have been made (25-27). Values of $G\left(\mathrm{H}_{2}\right)$ for slurries irradiated in in-pile tests were about 0.8 for concentrations of thorium-uranium oxides (2.8\% U, 93\% enriched in $\mathrm{U}^{235}$ ) of 285 to 850 grams per kilogram $\mathrm{H}_{2} \mathrm{O}$. Average particle diameter for the slurry used in these tests was between 1 and 3 microns. Studies of recombination catalysts compatible with the slurry are being carried out; molybdenum oxide looks promising. Range-energy relationship for fission recoll fragments In a slurry reactor, some fraction of the fission fragment kinetic energy will be lost in the solid. The fraction whlch escapes the solld is available for decomposing the 
water. Moreover, only the fraction which escapes the solid can be useful for promoting chemical reactions in process reactors. If the rate of energy deposition were known, the fraction of energy escaping the solid could be calculated. Unfortunately, this relationship is so complex that approximations must be used. In the calculations that have been made (28-32), two approximations have been used: Stopping power, $\mathrm{dE} / \mathrm{dx}$, was elther assumed to be a constant over the path length, or it was assumed to vary inversely with the velocity of the fragment. In the most complete study to date, Sears and Steinberg have calculated for both of the above approximations the fraction of energy that is deposited in the fluid medium as a function of both particle size and concentration. In addition, they have allowed for the case in which $G$ varies with the rate of energy deposition as shown by Edwards and Moseley (33). In order to calculate the fraction of energy lost in neighboring solid particles, that is, the concentration effect, the approximation that all the fission fragments escaped the parent particle with the same kinetic energy had to be made (34).

Little data are available for comparison with these calculations. Several investigators $(35,36)$ have measured the distribution of fission products between the solid and liquid phases in irradiated slurries, but, because of the small particle size and large surface area, adsorption effects were predominant. By adding active charcoal to their slurry, Hermans and Plas (37) were able to obtain distribution data as a function of particle size and concentration. 


\section{THEORY}

\section{Introduction}

In this section the relationship between the fraction of fission recoll energy deposited in the fluid and the physical characteristics of the slurry will be calculated. The results w1ll then be used to correlate experimental data because there was no way for measuring this fraction. The work of Sears and Stelnberg (32) could have been used, but it was not known how well their models described the actual relationship. Since they used two models for the range-energy relationship, one does not know which model, or what combiration of the two, best describes the actual situation. Furthermore, there was no basis for their use of average energies in determining the concentration effect, except that it was about the only way that the calculation could be done by hand.

$$
\text { Since there are very little data avallable on slurry }
$$
systems, this calculation must be based on fundamental data and theoretical considerations. Before this relationship can be calculated, it is necessary to know the range of the fission fragments and their rate of energy loss. Range-energy relationship

The stopping power may be defined as $-d E / d x$, that is, the rate at which the kinetic energy of the particle is lost to the medium along the path of the particle. According to Bohr (38), the nature of the stopping process is 
dependent on the velocity of the moving particle. If the velocity is greater than the orbital velocities of all electrons, the energy loss is mainly by interaction with the electrons of the stopping medium. Bohr proposes the velocity of the electron in the hydrogen atom as roughly the dividing line between stopping by atomic interactions and stopping by electronic interactions. By the time that the light and heavy fission fragments have slowed down to this velocity $\left(2.2 \times 10^{8} \mathrm{~cm} / \mathrm{sec}.\right)$, they have lost 98 and 95 per cent of their respective energies. The stopping power for most of their energy loss is therefore due to electronic interactions.

Theoretical expressions for the stopping power predict that it depends upon the charge and velocity of the particle and the nuclear charge, effective ionization potential, and atomic density of the stopping medium $(38,39)$ :

$$
-\frac{d E}{d x}=2 \pi e^{4} z^{a}\left[\frac{N Z}{E}\left(\frac{M}{m}\right) \ln \left(\frac{4 E}{I}\right)\left(\frac{m}{\bar{M}}\right)\right]
$$

Here $\underline{m}$ and $\underline{e}$ are the electron mass and charge, $\underline{M}$ and $\underline{E}$ the mass and energy of the moving charged particle, $\underline{z}$ the number of unit charges carried by the particle, NZ the number of electrons per unit volume of irradiated material, and I the parameter characteristic of the material. In a very simplified form, the stopping power is directly proportional to the square of the charge of the particle and inversely proportional to its energy. For a constant charge on the 
particle, the stopping power should increase as the particle slows down. However, as the highly charged fission fragment travels through the medium, it plcks up electrons. Since the net charge on the particle decreases, the stopping power due to the charge decreases. Theoretical expressions for the stopplng power for the fission fragments are therefore quite complex.

One expression is obtained by assuming that the stopping power is constant throughout the path length. Let $x$ be the distance at which the particle has energy $E$. Then $R$ minus $x$ is the range of the particle with energy $E$. The range-energy relationship can then be expressed as follows:

$$
(R-x)=\int_{0}^{E}(-d E / d x)^{-1} d E=\int_{0}^{E} C_{I} d E=C_{1} E
$$

From initial conditions of $E=E_{0}$ at $x=O, C_{1}=R / E_{0}$ Solving for the fraction of energy lost, $E / E_{0}=(1-x / R)$ 。

Another approximation may be obtained by assuming that the stopping power varies inversely with the velocity, that is, with the square root of the energy. This relationship is then:

$$
(R-x) \int_{0}^{E} C_{2} E^{-1 / 2} d E=C_{3} E^{1 / 2}
$$

From initial conditions, $\mathrm{C}_{3}=\mathrm{R} / \mathrm{E}_{\mathrm{O}}^{1 / 2}$. Thus, $E / E_{O}=(1-x / R)^{2}$

The actual relationship is somewhere between these two. 
Alexander and Gazdik (13) have shown that the empirical equation: $(R-x)=C_{4} E^{2 / 3}$ gives an adequate fit to the experimental data for both light and heavy fragments from their initial energy to about one-half the initial value. Taking $\mathrm{C}_{4}=\mathrm{R} / \mathrm{E}_{0}^{2 / 3}$ from initial conditions, the equation becomes $E / E_{O}=(I-x / R)^{3 / 2}$. This relationship will be the one used in calculating the fraction of energy lost in the fluid of the slurry. Range of the fission fragments

The ranges of fission recoil particles have been measured in only a few materials. In order to determine the ranges in other materials, use is made of the fact that stopping power is a function only of atomic properties and the density of the medium. Upon multiplying the range by the density, 1.e., $R p=R^{\prime}$, an expression for range is $o b-$ tained that is a function only of atomic properties.

Ranges in other elements may be approximated by using the following formulas $(38-41)$ :

$$
\begin{gathered}
R^{\prime}{ }_{1} / B_{1}=R^{\prime}{ }_{2} / B_{2} \\
B=0.60 A_{s}\left(\frac{A_{s}+A_{r}}{A_{r}}\right) \frac{\left(Z_{s}{ }^{2 / 3}+Z_{r}{ }^{2 / 3}\right)^{1 / 2}}{Z_{s} Z_{r}}
\end{gathered}
$$

Here $\underline{Z}$ and $\underline{A}$ are atomic and mass numbers with subscripts $\underline{\underline{S}}$ for the stopping atoms and $\underline{r}$ for the recoiling atoms. Although these equations have been derived for velocities 
less than that of the hydrogen electron and for low atomic weight media, they are used here for lack of appropriate theory. As long as the atomic weights of the two stopping elements--known and unknown--are of the same magnitude, the error in the calculated range should not be significant. Table 1 gives the range values needed in this work as calculated from the measured ranges in hydrogen, aluminum, and uranium $(11,42,43)$. The range in oxygen was calculated from that in aluminum; that in thorium, from that in uranium. The range in a mixture was estimated from the relationship that the inverse range in the mixture is equal to the sum of the inverse ranges of each of the elements weighted by their respective mass fraction.

Observe in Table 1 that the range of the heavy fragment in any material is about eight-tenths the range of the light fragment. The result of this characteristic is that the ratio of the range in one material to that in another is a constant for both fragments. This dimensionless ratio will be used as a parameter in calculating the fission-fragment energy distribution in the slurry. Physical properties of the slurry

Density of the solid, density of the fluid, particle size, concentration, and distribution of the solid are characteristics of the slurry that must be known before calculation of the energy distribution can ide made. The densities will be used to convert the values of range given 
TABLE 1

RANGES OF FISSION FRAGMENTS

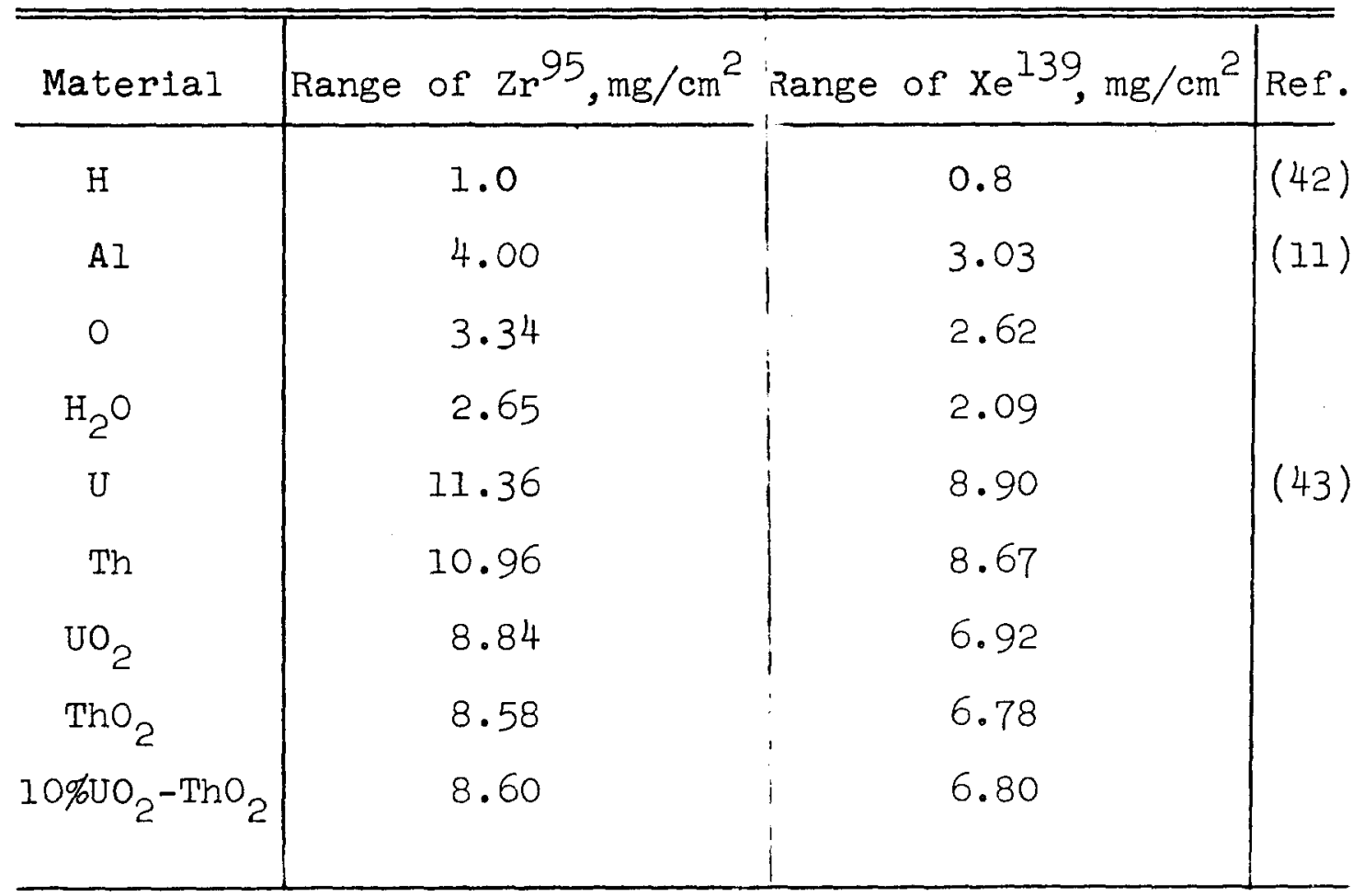


in Table 1 to units of length. It is the ratio of ranges in these units that will be used as a parameter. Since the range-energy relationship depends upon the ratio of the distance travelled to the range, the ratio of the particle diameter to the range in the solid will be used as a parameter. The probability of flssion fragments entering neighboring particles is a function of the volume occupled by the solid; the concentration parameter will therefore be the volume fraction of solid. No particular distribution of the solid will be assumed; only the number of particles per unit volume will be used. Movement of the particles with respect to time is not a variable, because the time during which the fragment is travelling through the medium is less than $10^{-11}$ seconds. Mechanics of the calculation

If it were not for the effect of neighboring particles on the fraction of energy lost in the solid, the energy distribution could be easily calculated using Tobias' (44) method of analysis. Because this effect may be significant, some other technique must be used. Estimates of this effect have been made $(32,34)$, but, since the approximation that all the fragments left the parent particle with the same energy was required, the accuracy of these results is questionable. A method which does not require this approximation is that called the "Monte Carlo" technique. High-speed 
digital computers must be used for the solution of the problem by this method.

Calculation of the energy distribution in the slurry has been done in this thesis by the Monte Carlo technique with the help of an IBM "704" computer. In the first step of the calculation, the computer program selects the distribution of the solid spheres in the slurry. It picks at random the coordinates of their centers, rejecting any spheres which overlap, unt1l the number required per unit volume is reached. Then at a random point in one of the spheres, two fragments of unit energy are sent out in opposite directions along the $x$-axis. The energy lost in the sphere is computed from the range-energy relationship. If a fragment escapes the sphere, the program calculates the residual range of the fragment in the fluid and computes the energy lost in the fluid. If the fragment strikes another sphere, the residual range in the solid is computed and the energy lost in that sphere recorded. This proced-. ure is repeated until the fragment has lost all its energy. The residual range of the fragment is assumed to be proportional to the two-thirds power of its remaining kinetic energy, but otherwise independent of the previous history of the fragment. After a large number of fragments have been followed, the results are averaged to give the fraction of energy lost in the parent sphere, that lost in the fluid, and that lost in other spheres. 
By using dimensionless variables throughout the problem, a generalized solution is obtained. The fraction of Inttial energy lost in the solid and liquid is given for three varlables: the ratio of the diameter of the particle to the range in solid, $D / R_{S}$, the ratio of the range in fluid to that in solid, $R_{f} / R_{s}$, and the ratio of the concentration of solid to the density of the solid, $\mathrm{C} / \mathrm{p}$, which is the volume fraction solid. The values of initial energy and range for both light and heavy fragments may be inserted to give dimensions to the final answer. Results of the calculation

The primary outcome of the calculation was the relationship of the fraction of energy lost to the fluid to the particle size and concentration of the slurry. Figure I gives the fraction of energy avallable for a range ratio of 3.0, while Figure 2 shows the effect of changes in this ratio. For zero diameter the values were calculated from the relationship for a homogeneous medium that the inverse of the range in any medium is equal to the sum of the inverse ranges of the components in the medium times their respective volume fractions. The energy fraction is that absorbed by the molecules of fluid, i.e.,

$$
\frac{\bar{E}_{0}}{E_{0}}=\frac{(I-C / \rho) / R_{f}}{(1-C / \rho) / R_{f}+(C / \rho) / R_{s}}=\frac{1}{I+\frac{C / \rho}{I-C / \rho}\left(\frac{R_{f}}{R_{s}}\right)}
$$




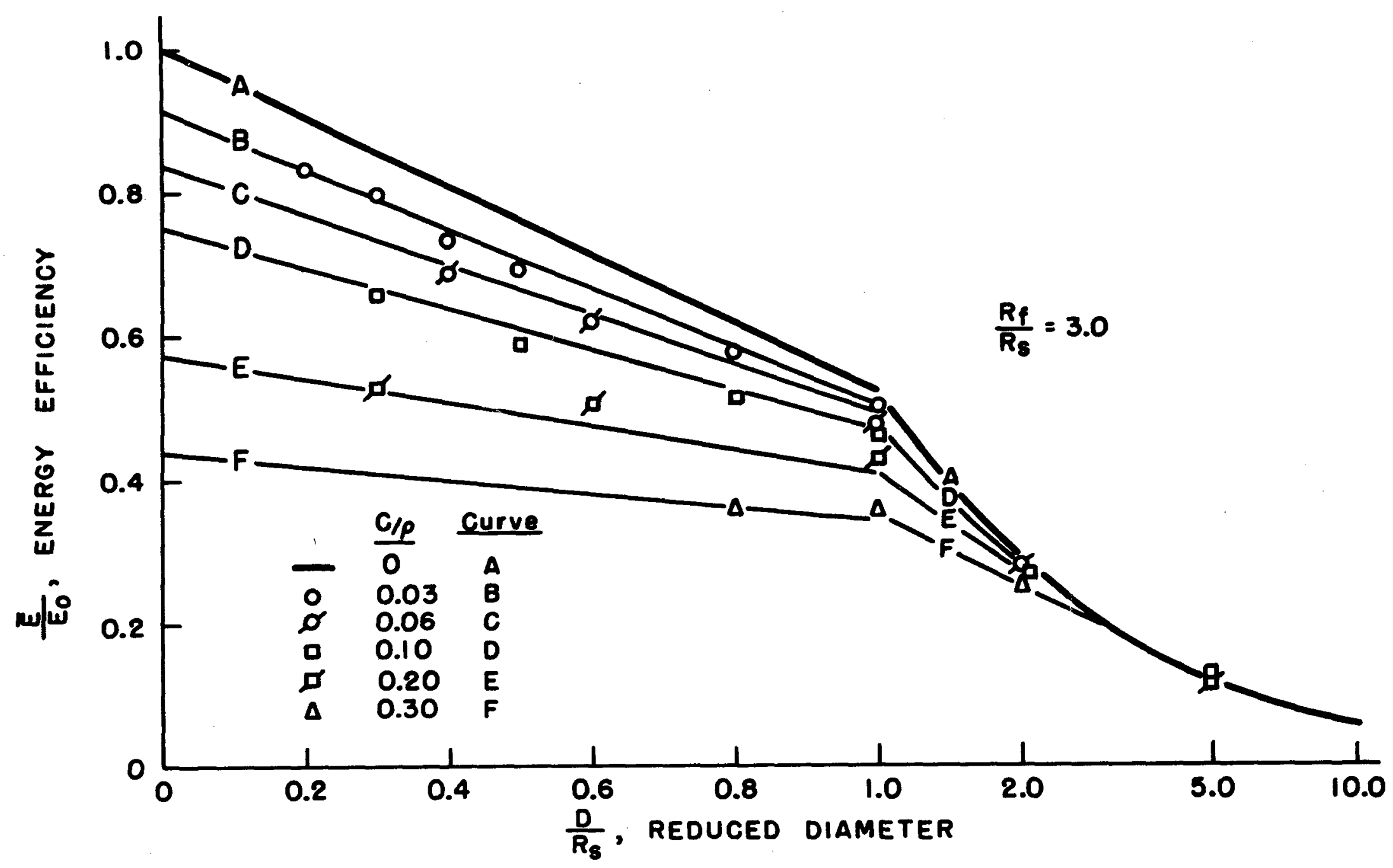

Figure 1. Effect on concentration $C / P$ on the energy efficiency $E / E_{0}$. 

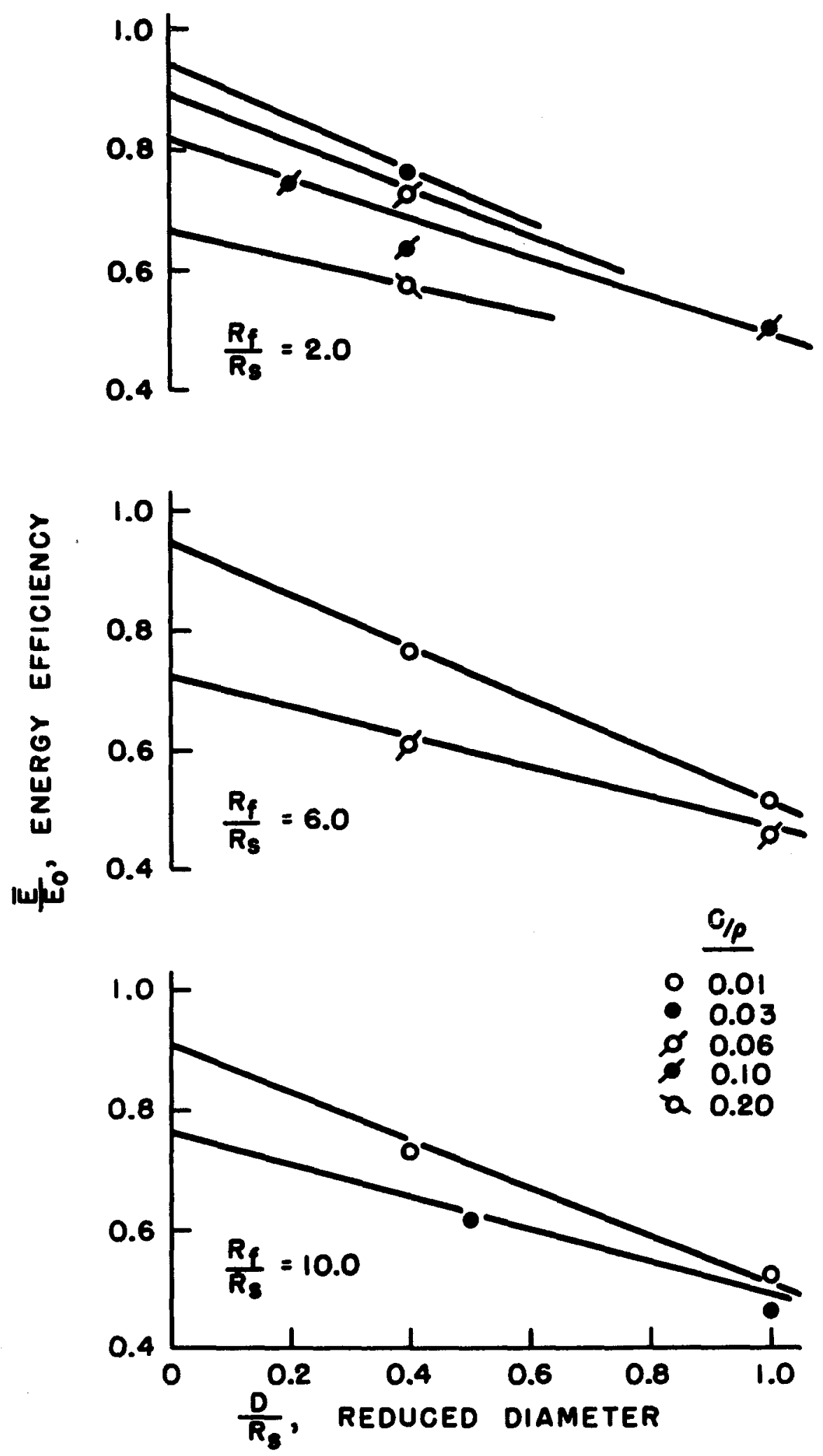

Figure 2. Effect of range ratio $R_{f} / R_{S}$ on the energy efficiency $\bar{E} / E_{0}$. 
The line for a concentration of zero represents the average fraction of energy that should escape from a single sphere. This is equivalent to that which escapes from the parent sphere, where parent is defined as the sphere in which the fission occurs.

Note that the results in Figures 1 and 2 have been correlated up to a diameter equal to the range with straight lines. The equation used for drawing these lines is the following:

$$
\frac{\bar{E}_{0}}{\bar{E}_{0}}=\frac{1}{1+\frac{C / \rho}{1-C / \rho} \frac{R_{f}}{R_{S}}}-0.48\left[e^{-I .8(\mathrm{c} / \rho)\left(R_{f} / R_{s}\right)}\right] \frac{D}{R_{S}}
$$

Equation (24) fits the computed data points well enough so that one should be able to use it to predict the energy efficiency for any slurry system. Since the effect of concentration decreases rapidly as the diameter increases above the range, an equation which will correlate this data is not really necessary. Interpolation between the curves in Figure I should give sufficiently accurate values.

Another outcome of the program is the fraction of particles which are stopped by solid as a function of the three parameters. Figure 3 shows the effects of particle size and concentration for a range ratio of 3.0 ; whereas Figure 4 shows the effect of the range ratio. For zero concentration the value was calculated from the probability of a fragment 


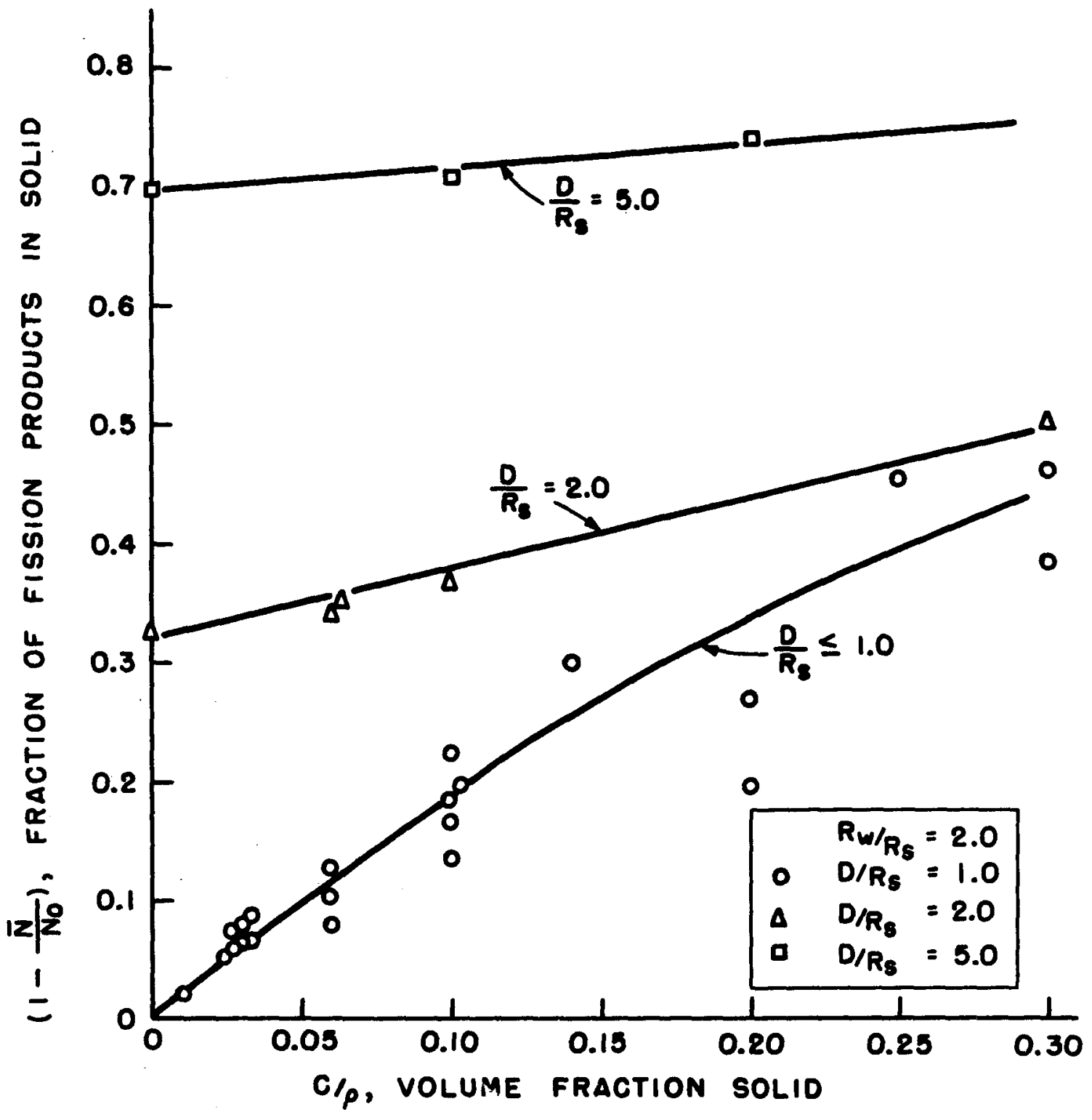

Figure 3. Froction of frogments stopped in solid. 


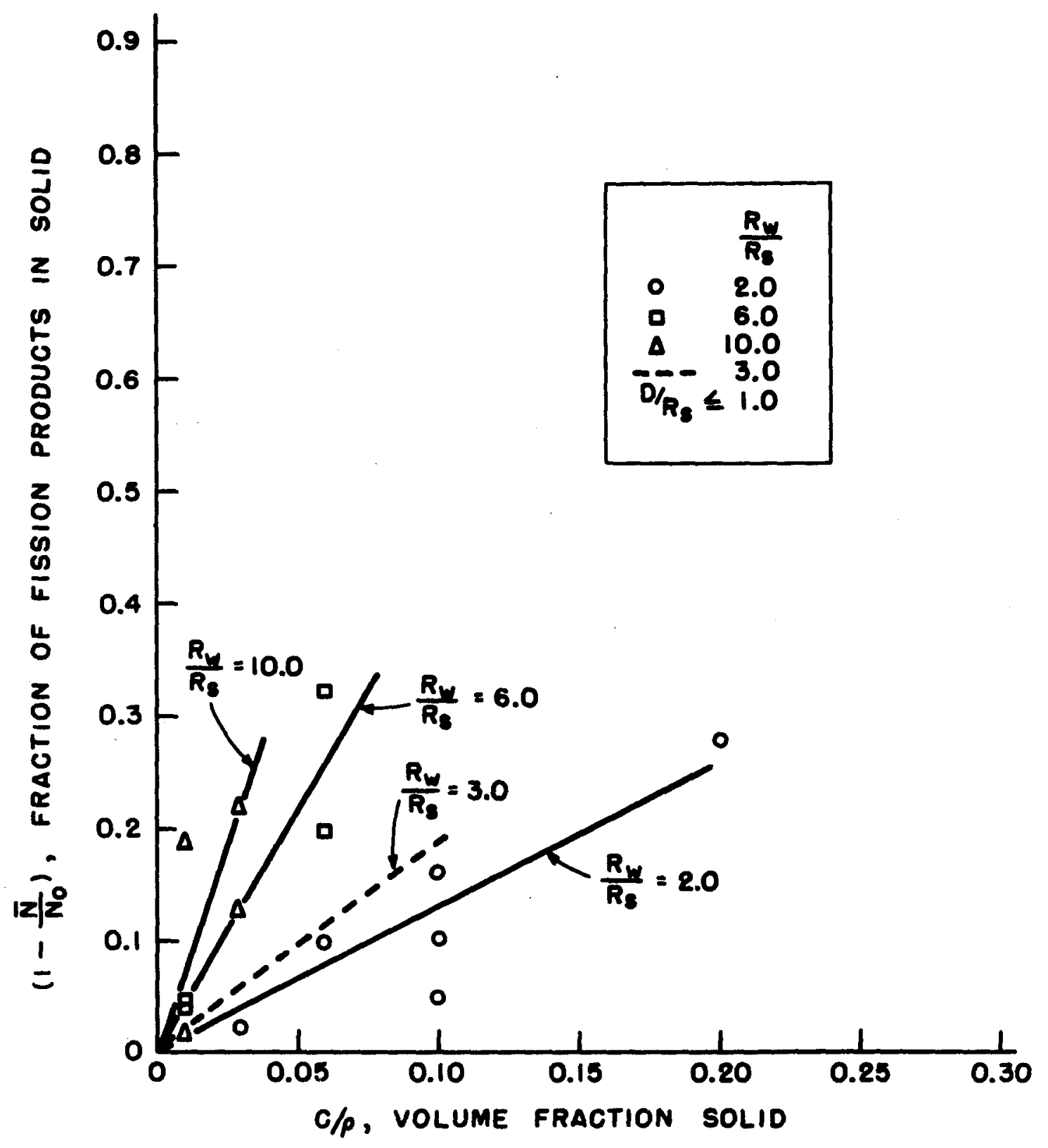

Figure 4. Effect of the range ratio on the fraction of frogments stopped in solid. 
escaping its parent. For a diameter less than the range all the fragments escape the parent; hence, no distinction was made between these diameters.

The program also recorded the energy of the fragments upon their escape from their parent. This is shown in Figure 5 as the frequency distribution of the energy of the fragments as a function of the particle size. The curves have been normalized so that the total area under any curve is unity.

A comparison of results of the computer program with the work of Sears and Steinberg (32) is shown in Figure 6. Since the line for the three-halves model is about halfway between those for the other models, as might be expected, the computer program is probably correct. 


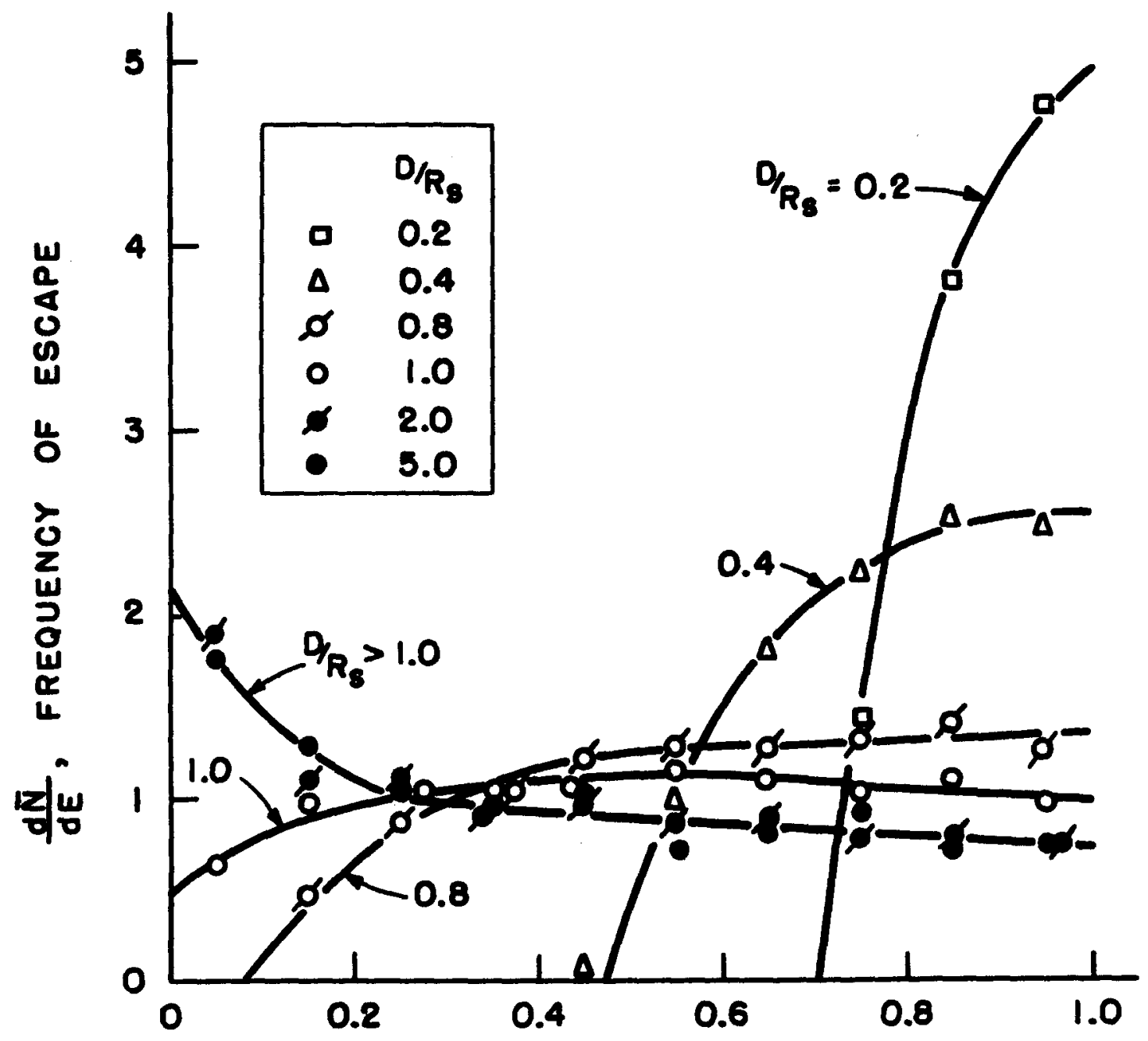

FRACTION OF INITIAL ENERGY ENTERING FLUIO

Figure 5. Energy spectrum of the fragments leaving the parent sphere. 


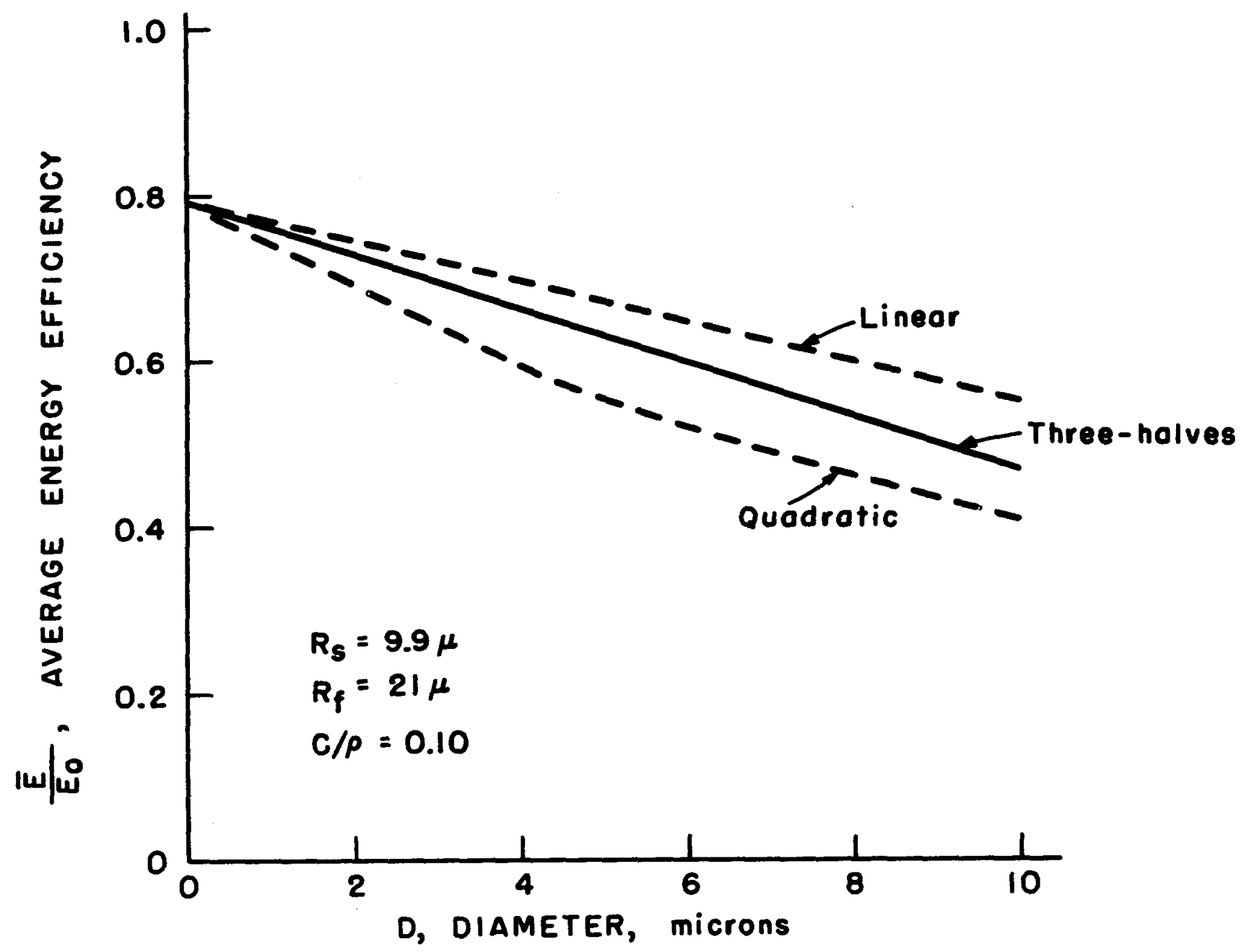

Figure 6. Effect of range-energy model on energy efficiency. 


\section{EXPERIMENTAL PROGRAM}

\section{General approach}

The purpose of the experimental program was to obtain the rate of water decomposition due to the energy of the fission recoll fragments. The fuel used in the program consisted of solid spherical particles containing 10 per cent $\mathrm{UO}_{2}$ in $\mathrm{ThO}_{2}$, because this was avallable at the time. In the previous section the relationship between the fraction of fission energy released to the water and the slurry particle size and concentration has been calculated.

Much of the equipment design depends upon the method chosen for measuring the effect of the fission recoils on the water. In previous work (5) measurement of the rate of increase in pressure during irradiation was used to indicate the rate of decomposition. In such cases the back reaction, recombination of hydrogen and oxygen to form water, cannot be prevented. Even in the presence of bromide ion, which is supposed to inhibit the back reaction, as given by equations (10) and (11), there was found to be an effect of the ratio of fission energy to background energy from the reactor. Another method for measuring the rate of water decomposition (7) is that of stripping the hydrogen and oxygen out of the solution during irradiation. Since the back reaction depends on the concentration of hydrogen in solution, recombination can be controlled. For this reason the latter method was used in this experiment. 
The decision on the method for measuring the decomposition had an effect on other variables. Since the volatile fission products would be stripped out with the radiolytic gases, the upper limit on the amount of U-235 that could be handled was determined by the radioactivity in the gas phase. On the other hand, there had to be a measurable rate of decomposition and enough U-235 to be able to distinguish the decomposition due to the fission energy from the other forms of lonlzing radiations. The actual values of the limits on the uranium content depend on the neutron flux available and the intensity of the gamma background. As a result of these conditions, the concentration variable was somewhat limited.

The design of the equipment was influenced by the magnitudes of these variables. Since the purpose of the equipment was to handle the gas bubbled through the slurry, the size of the equipment depended on the flow rate. But the flow rate had to be large enough to strip out the hydrogen, yet small enough that the hydrogen in the gas could be. measured. Keeping the slurry in suspension and bubbling gas through it during irradiation in the reactor placed additional requirements on the equipment. Discussion of the problems and the steps taken to solve them will be given in the sections on particular pieces of equipment. 
Reactor facilities

For this experiment the apparatus located in a neutron fleld had to satisfy the following requirements: (1) There must be some way of passing gas through the sample during the Irradiation. (2) There must be some way of keeping the slurry in suspension during the irradiation. (3) There should be some means of removing the sample from the radiation zone after the irradiation, so that induced activity is kept to a minimum. In addition to these requirements, the ratio of gamma to neutron flux must be low enough that the water decomposition due to the fissions can be measured.

In discussing these requirements with the Reactor operations personnel at Argonne, it was decided that the best facility available was the thermal column of $\mathrm{CP}-5$ reactor. Six inches of lead between the reactor qore and the thermal column absorbed most of the core gamma rays. Calculations (45) of the neutron and gamma flux Indicated that the ratio of neutrons to gammas may be satisfactory at the high intensity end of the column; the ratio decreases as the distance from the core increases. An available shielding de.vice, could be used for inserting and removing the sample while the reactor was at power.

The final design of the equipment had to meet the following requirements of the Reactor Operations Safety Committee: (1) The gas passed through the slurry must be under 
a negative pressure, so that, in the event of a leak, a1r would leak into the system rather than fission gas out. (2) Any solid or liquid should be prevented from getting loose inside the column. (3) Water must be carefully contained because a spill could shut down the reactor. (4) Mercury may be used only if adequate precautions are observed, because loose mercury could get inside the core and amalgamate with the aluminum fuel cladding. (5) Members of the commlttee must observe the first experiments. Figure 7 is a sketch of the CP-5 reactor showing the location of some of its facilities. The thermal column, a horizontal hole, has a removable section of graphite 12 in. $x 12$ in. $x 48$ in. long. Blocks of graphite, 4 in. $x 4$ in., may be removed to allow space for equipment. Figure 8 is a sketch of the beam catcher, the shielding device for positioning the sample during irradiation. At the extreme end of the movable 4 in. $\times 4$. in. beam, which contains all the access tubes and wires, was mounted the equipment to be irradiated. The shield itself is mounted on wheels that run on a track. Once the shield is aligned, it can be moved back and forth on the track without getting out of line with the hole.

The equipment for containing the slurry must be designed to utilize these facilities. It must be able to fit into a horizontal hole no larger than 4 in. $x 8$ in. crosssection, because the shielding is inadequate for larger holes. 


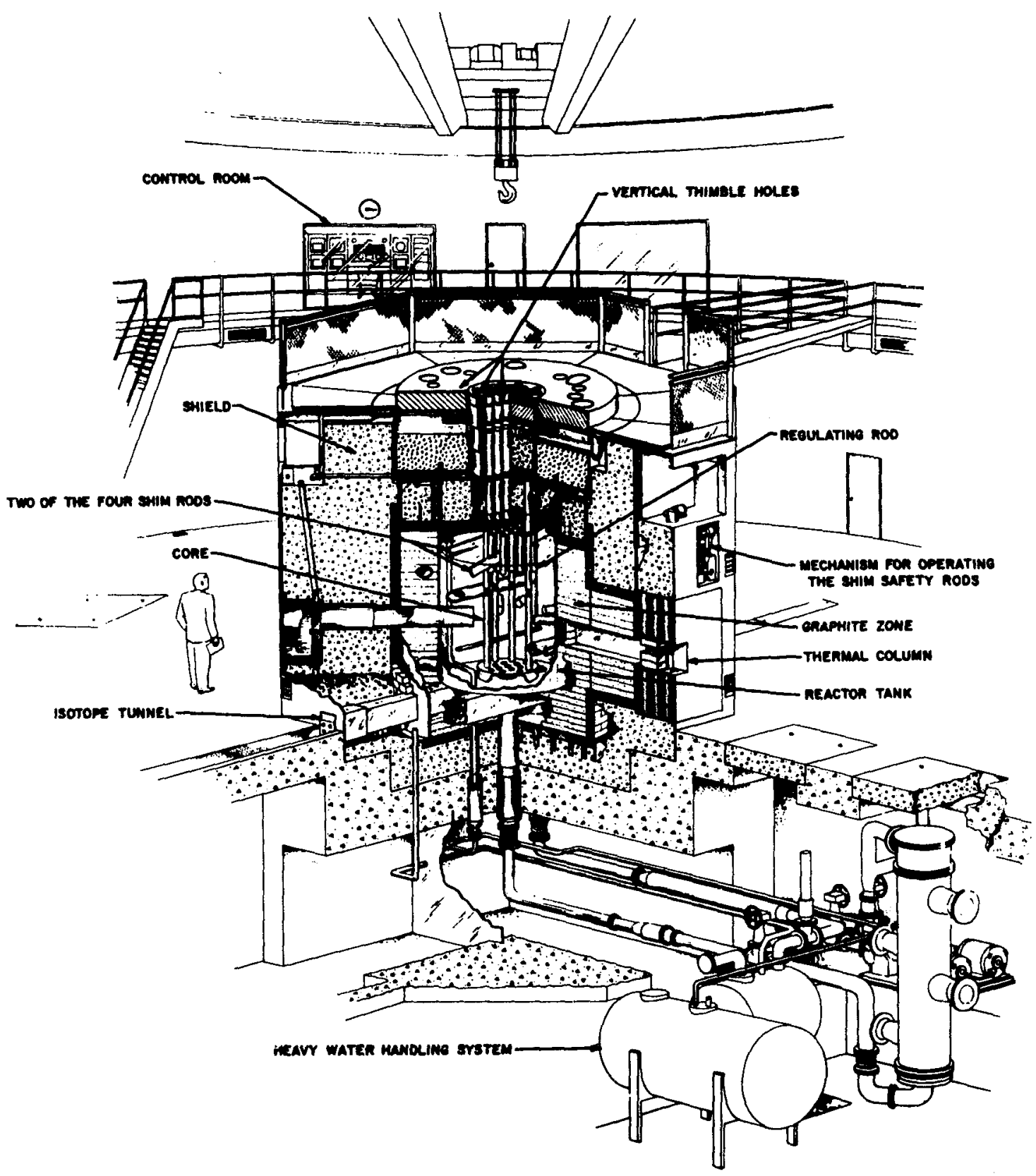

Figure 7. Diagram of the CP-5 Reactor Facilities 


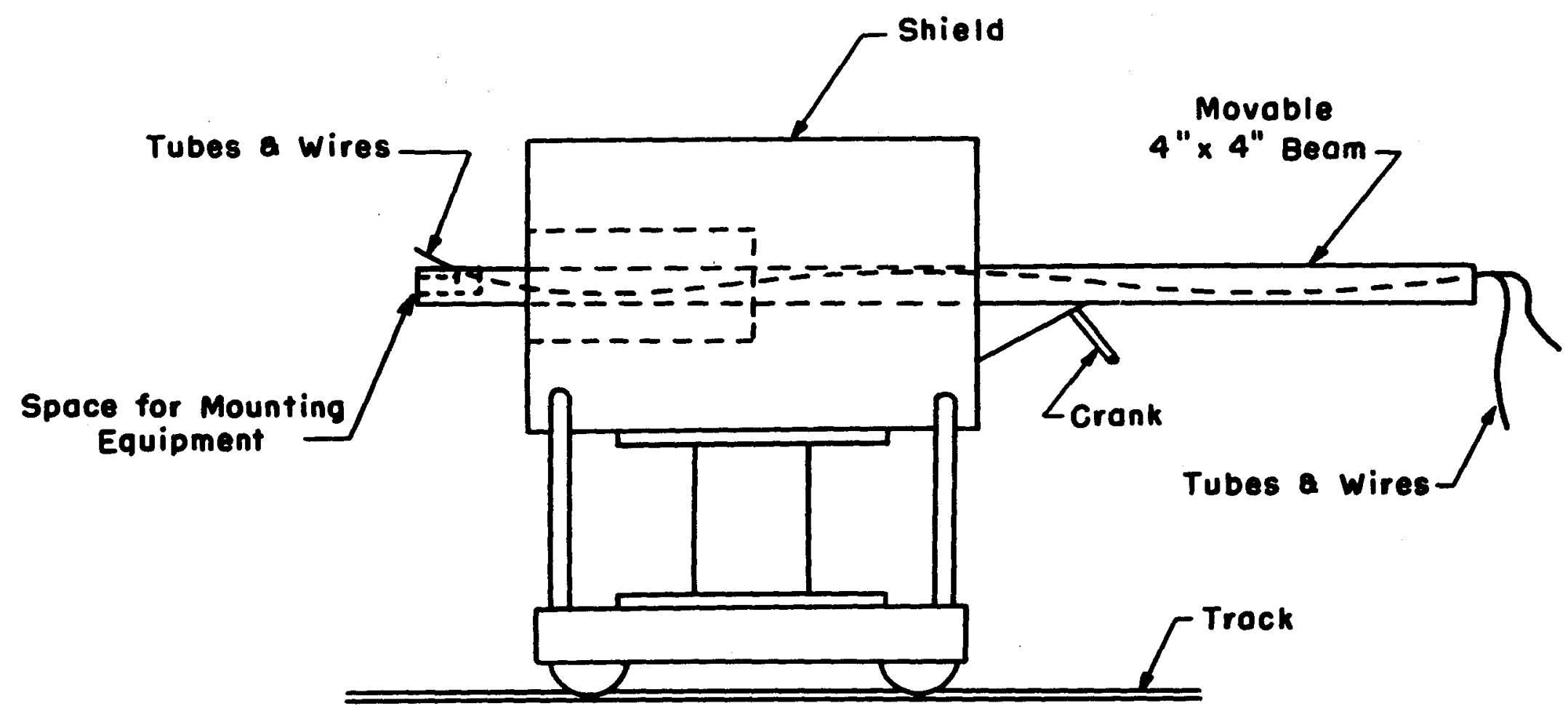

Figure 8. CP-5 thermol column beam catcher. 
The length of the extension must be 48 inches; any other length would e1ther not reach the end of the thermal column, or the beam catcher could not fit against the face of the reactor when the thermal column gate was closed. Reactor irradiation vessel

The basic requirement in the design of this equipment was that the slurry must be kept in suspension during the irradiation. Among the choices of methods were bubbling with a gas, fluidization with clrculating water, shaking mechanically, magnetic stirring, and mechanical stirring. In the first method such a high flow rate of gas would be necessary that the radiolytic gases would be so diluted that analysis would be difficult. The circulating water system had the disadvantage that it required a relatively large amount of water compared to the size of the sample. The distribution of the slurry and the stripping action of the gas was believed to be better under stirring conditions than under shaking. To stir magnetically would require a large mass of metal in a region of high neutron flux. By stirring mechanically, the drive mechanism could be located some distance from the vessel in a region of low flux. The design of the equipment used in this experiment for stirring the slurry is shown in Figures 9 and 10 . It consists of a horizontal arive mechanism at one end and a vertically stirred vessel at the other. The best method of transmitting the torque was considered to be straight shafts 

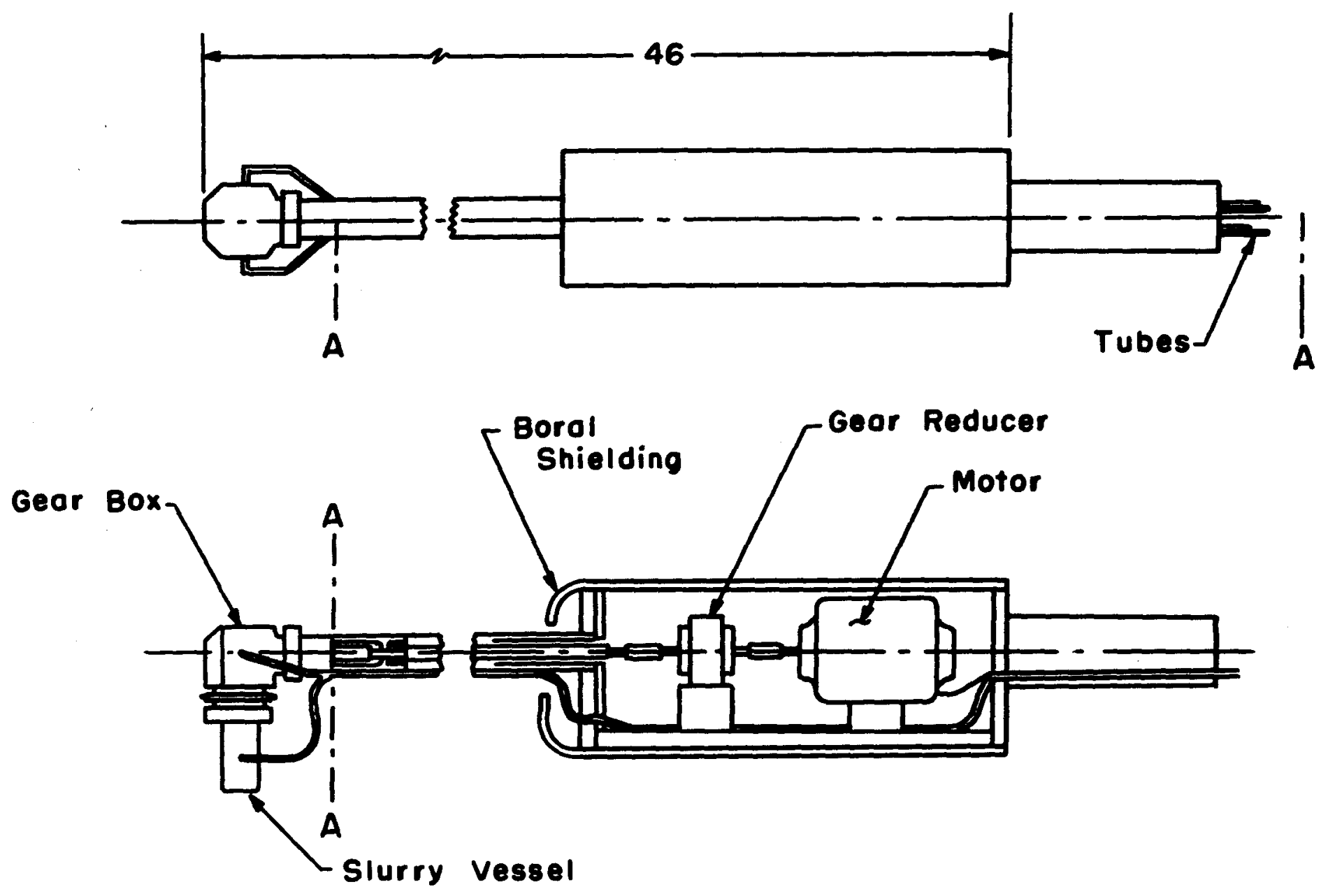

Figure 9. Reactor irradiation vessel. 


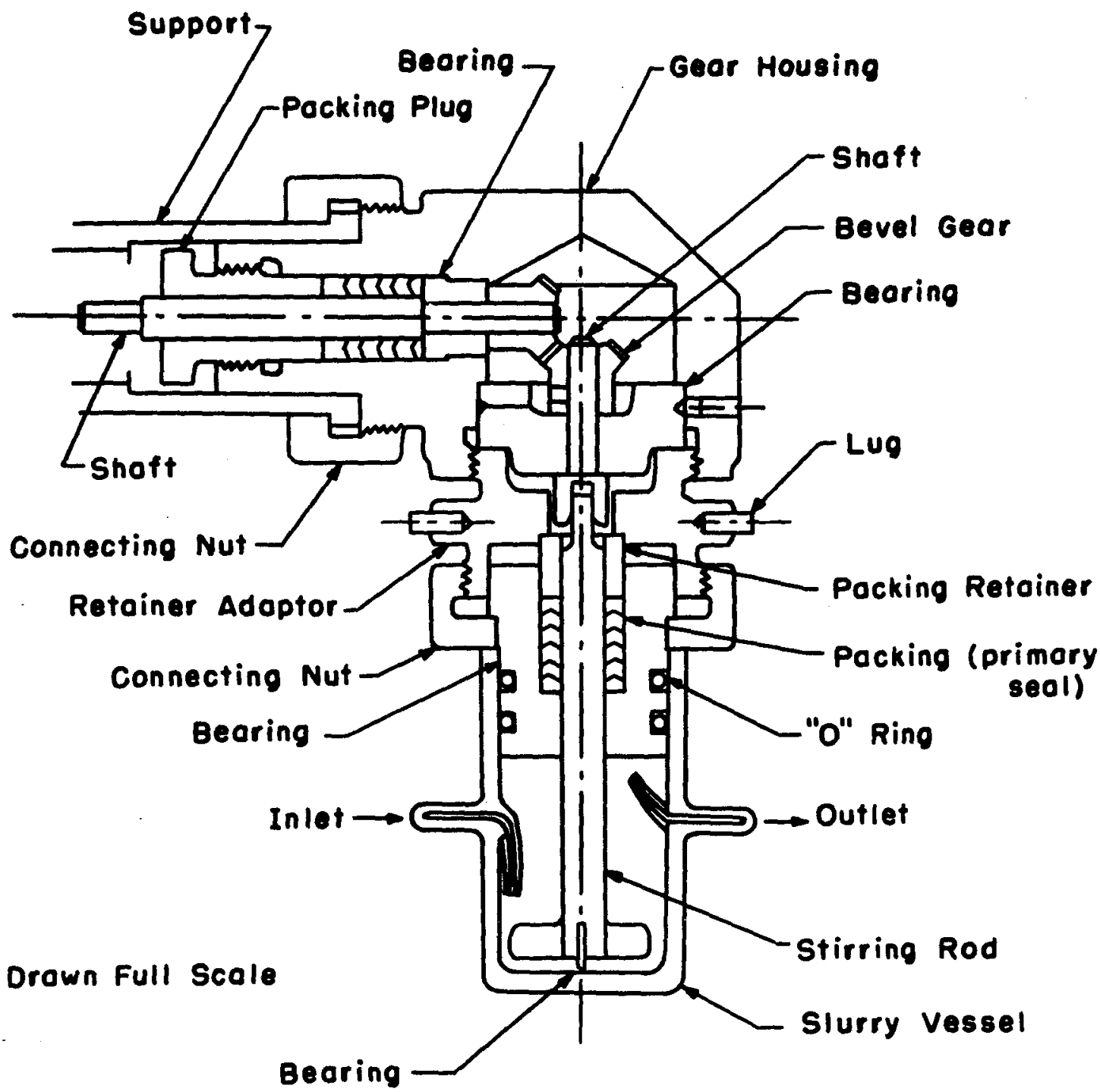

Figure 10. Slurry vessel and gear box assemblies. 
coupled with a pair of bevel gears. Seals were required in two positions along these shafts. The primary seal separated the gas bubbled through the slurry from that above the seal. Since it was desired to measure the radiolytic gases In the absence of a1r, the space above the primary seal was purged with the same gas used for the bubbling; therefore, a second seal was necessary for the purge system. Because of the radioactivity of the slurry and the equipment, it was required that the vessel containing the slurry be easily, quickly, and safely attached to and removed from the rest of the apparatus. With these general characteristics the design of the equipment evolved to that shown in the Figures 9 and 10 .

Materials of construction were very important because the equipment was going to be exposed to a high neutron flux. Fortunately the flux falls off by a factor of about ten per foot in the column, thus permitting the use of a conventional drive mechanism. The motor, a Bodine, 1/16 h.p., 5,000 rpm, series-wound universal type, coupled with a Metron series 10A, 5 to 1 , speed changer provided a maximum shaft speed of 1,000 rpm. Since this drive system would still be located in a neutron flux of about $10^{9} \mathrm{n} / \mathrm{cm}^{2} / \mathrm{sec}$ during the irradiation, it was enclosed in a $1 / 4$ inch thick shield of boral. Most of the supporting structure was made of 6061 aluminum, but that which was located in the highest flux region was made of micarta. 
The shaft in the second seal was made of Zircaloy-2 because aluminum was found to be too soft. Graphite was used for all the bearings. Teflon ("Chemlon" by Crane Packing Co.) was chosen for the packing materlal for the seals; however, because it does not stand up well under radiation, the seals were designed to permit easy replacement of the packing. The bevel gears had to be made of aluminum (Pic Design Corp.); brass or steel would have become too radioactive. The material chosen for the vessel containing the slurry and its stirring rod was quartz, because it is chemically inert and has a small, short-lived induced activity.

There are four tubes for handling the gas passed through the equipment. By using 1/16 in. I.D. aluminum tubing for the gas bubbled through the slurry, the time it takes for the radiolytic gases to reach a steady-state concentration is short. Since this is not important for the purge gas, $1 / 8$ in. I.D. aluminum tubing is used for it. These tube sizes are continued through the beam of the shield. The $1 / 4$ in. O.D. polyethylene tubing, cemented to the gear box, is fastened to the aluminum tubing with a short piece of mbber tubing. This type of connection is also used to fasten the small aluminum tubing to the quartz tube of the slurry vessel. To disconnect these gas lines, one simply cuts the rubber section.

The most important part of this apparatus was the slurry vessel assembly, which is sketched in Figure 10. 
Actually, three of these units were used in the experiment--while one was being irradiated, the other two were being emptied and refilled. Two I in. O.D. Buna-N "O" rings make the seal between the slurry vessel and the bearing, and they also serve to hold the vessel to the rest of the unit. The vessel was designed with a wide opening so that the highly radioactive slurry could be easily removed from the vessel after an irradiation. When the vessel is filled to the level of the tubes on the outside, it contains about $10 \mathrm{cc}$. of liquid; that leaves about $5 \mathrm{cc}$. for the vapor phase. These tubes were located far enough below the bearing that distortions in the wall did not interfere with the seal. The inlet tube is so placed to let the gas bubble through the slurry. The outlet gas is taken from a place near the top of the vessel and away from the wall where there is less probability of liquid being splashed into it. The top $1 / 4$ in. of the stirring rod was ground down to an $1 / 8$ in. thick tongue. The rest of it was ground to $1 / 4$ in. diameter to fit inside the packing. The single propeller was sufficient to keep the slurry in suspension and provide enough mixing to strip out the hydrogen. The 1/16 in. diameter by $1 / 4$ in. Iong rod of Teflon placed in the hole in the bottom of the stirring rod served as a bearing between the stirring rod and the vessel bottom. The rest of the assembly, consisting of the packing, packing retainer, retainer adapter, and connecting nut, was used to make the seal around the stirring rod. 
Figure 11 is a plcture of the reactor irradiation vessel mounted in the beam catcher. Figure 12 is a picture showing the end of the equipment in which the slurry is located. Gas collection equipment

Since the radiolytic gases were to be stripped out durIng the Irradiation, the purpose of this equipment was to handle the gas system. Actual design of the equipment depended on the gas used for stripping out the products and the method of analysis. Both nitrogen and argon could be used with the gas chromatographic method of analysis, which is discussed in the section on analytical methods. In addition, neither of these gases interferes with the decomposition of water. Nitrogen was chosen for the gas to be used in this experiment because there would be nc radiativity induced in it.

Sampling method, integral or differential, also influenced the design of the equipment. For an integral sample all the radiolytic gases formed during the irradiation are collected; yield of hydrogen is based on the total dose. On the other hand, a differential sample is collected over a short interval of time during the irradiation; yield of hydrogen is based on dose rate and flow rate. It was realized that the integral method might require more experiments and longer irradiations than the differential to accoung for the beginning and end effects of the irradiation. 


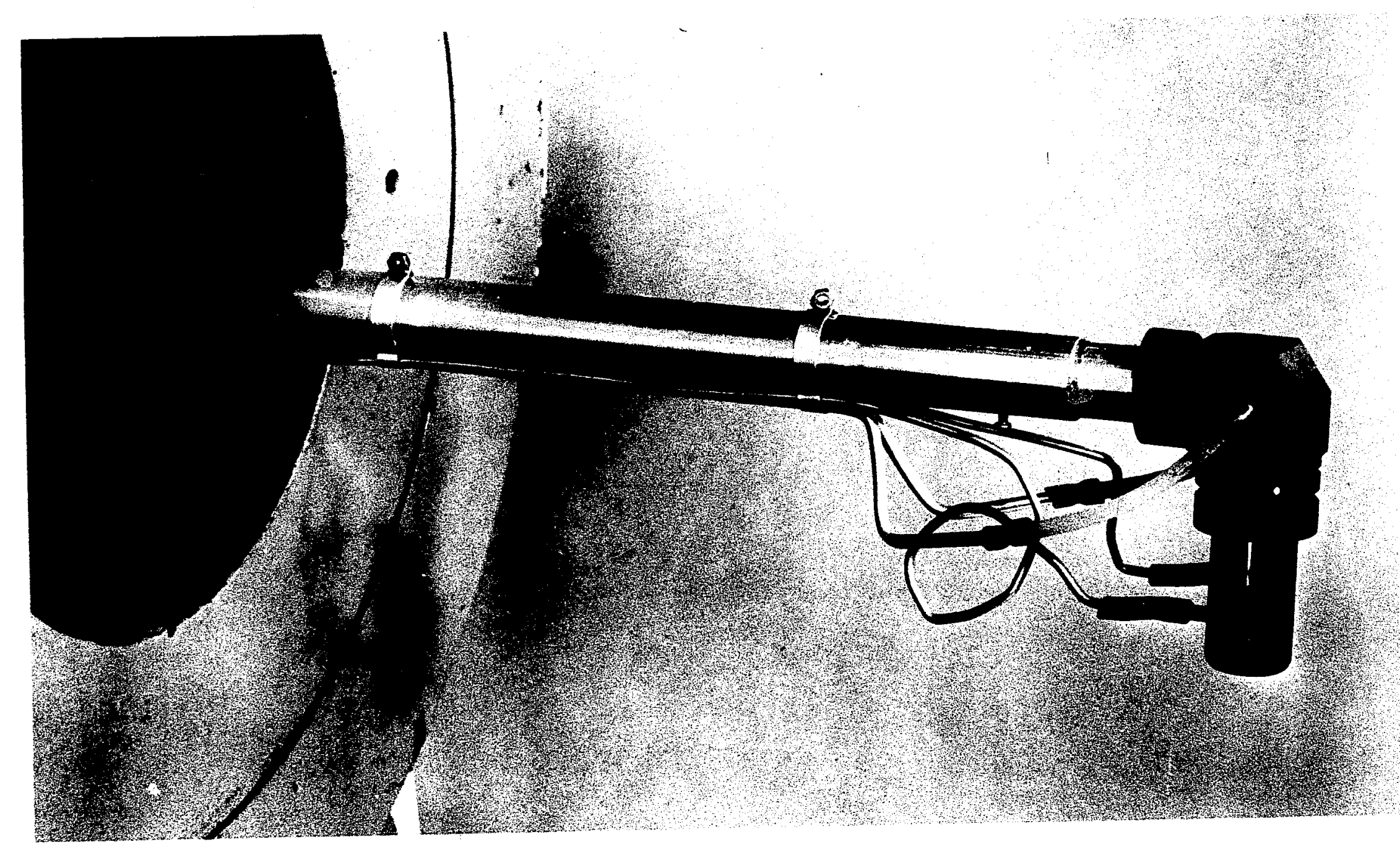

Figure 11. Photograph of the Reactor Irradiation

Vessel Mounted in the Beam Catcher 


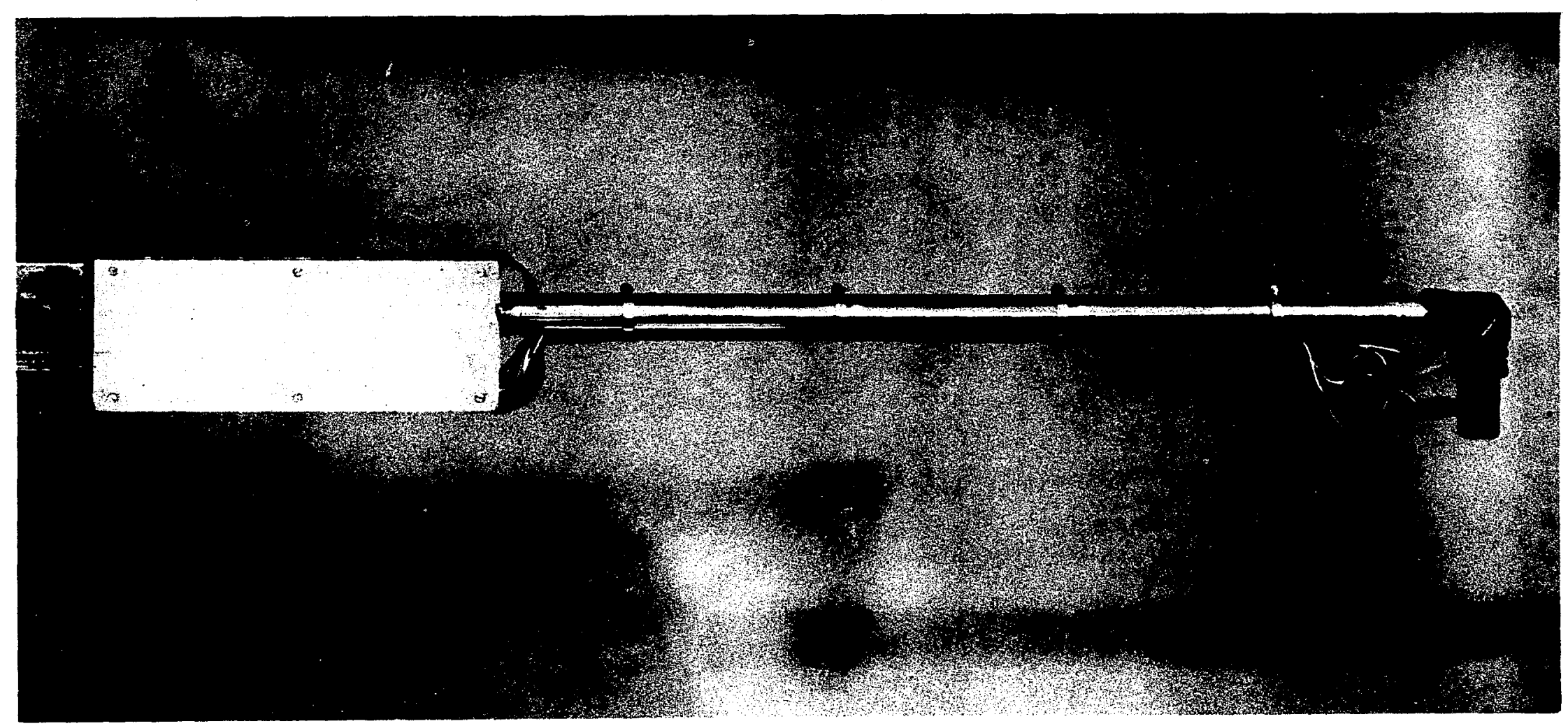

Figure 12. Photograph Showing the Position of the Vessel

When Withdrawn into the Beam Catcher 
For this reason the differential sampling method was chosen.

General characteristics of the gas collection system were that the nitrogen be passed through the slurry during the irradiation at less than atmospheric pressure, there should be some means for collecting samples of the gas stream, and the flow rate should be known accurately. Since the volatile fission products would be stripped out with the radolytic gases, it was desirable to provide some means for decontaminating the gas stream. The fission products in the gas were not altogether undesirable because, by monitoring their activity in the gas, some indication of the process inside the reactor could be gained.

Figure 13 shows a flow diagram for the gas collection equipment used in this experiment. Source of nitrogen was a standard compressed nitrogen cylinder equipped with a regulator valve. After the stream is divided, one line for the purge gas, the other for the stripping gas, each stream is passed through a Manostat Model FMIO42B flowmeter. Because of the difficulty in reading this type of flow meter accurately, these were used only to indicate the approximate gas flow rate. The manometer, about one meter long and a half-filled with water, was used to measure the vacuum in the gas lines and also served as a pressure relief valve. After passing through the gear box of the irradiation vessel, the purge gas was elther allowed to escape to the atmosphere 


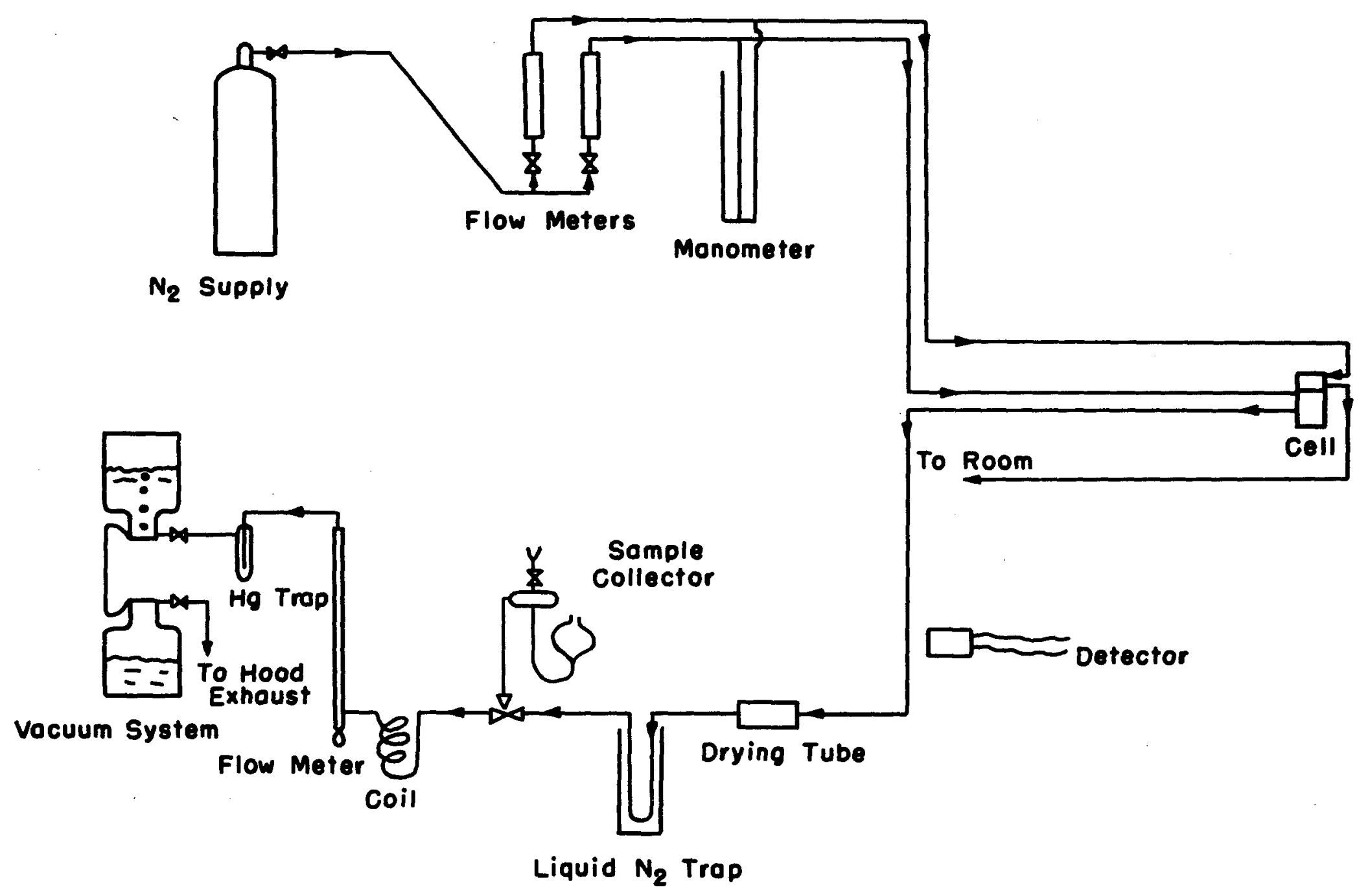

Figure 13. Flow diagram of gas collection equipment. 
or collected under vacuum. After the stripping gas came out of the reactor, it passed by the activity monitor, a two inch sodium iodide crystal with a photomultiplier. Excess moisture was then removed by Drierite in the drying tube. Volatile fission products were condensed in the liquid nitrogen trap. Duplicate tubes and traps were provided so that, if one of the units plugged during a mu, the flow could be switched to the second without interfering with the run. These units were made of Pyrex equipped with ball-and-socket joints, which permitted any one unit to be removed for cleaning. The purpose of the coll of copper tubing was to allow the gas to reach room temperature before it passed through the soap-film flow meter (46). The flow was measured accurately through the meter by timing the soap film over $10 \mathrm{cc}$ 's displacement.

Since the pressure on the stripping gas had to be less than atmospheric, the purpose of the rest of the equipment was to maintain that pressure during the run. The unit, consisting of one four-liter flask inverted over another, was designed to collect the gas by displacement of water in the top flask. Vacuum on the gas system was maintained by the liquid, leg between the top and bottom flasks.' The water, which was being displaced by the gas in the top flask, forced the gas in the bottom flask into a plastic bag. The contents of the bag, which included some fission products, 
was later exhausted to a hood. Design of the unit was such that, when the top flask had fllled with gas, the unit could be inverted and the collection continued for the next run or, If necessary because of a leak, for the same mun. The mercury trap served two purposes: It was used to adjust the pressure in the gas stream, for the liquid leg in the collection unit was not adjustable. It also prevented the loss of the liquid leg at the end of a run. As soon as the gas on the high-pressure side of the trap reached atmospheric, gas collection would automatically stop--all the nitrogen would then flow through the purge line.

The sampling apparatus, located just after the liquid nitrogen trap, was designed to collect about $5 \mathrm{cc}$. of the gas without affecting the flow rate through the slurry. Actual design of this unit depended on the technique used for transferring the sample to the analytical equipment. It was convenient to transfer the sample in a bulb that had been evacuated, for then a portion of it could be transferred under mercury to the chromatograph. Discussion of this analytical procedure will be found in a later section. The bulb consisted of a Pyrex tube, sealed at one end and fastened to a stopcock at the other. A short length of tubing connected a 5/20 ground glass joint to the other side of the stopcock. While the bulb was on the vacuum line, this stem of the bulb could be filled with mercury by using a Topler pump. It was essential then to collect the samples 
under mercury. Figure 14 is a sketch of the sampling unit and the transfer bulb. Before the collection of a sample the leveling bulb would be positioned so that the pressure on both sides of the three-way tap were equal. A large sized leveling bulb was used to minimize the effect of change in level of mercury during the collection of the sample.

Gamma irradiation facility

In order to try out the method of measuring the hydroen yields, the equipment was first tested in a 3,000 curie co 60 $\gamma$-irradiation chamber (47). Moreover, there was no problem of induced radioactivity as in the reactor. Such a facility also was used to determine the effect of the presence of the slurry on the dose of gamma radiation received by the water. The facility was equipped with access tubes so that gas could be passed through the slurry during irradiation. There was also a flexible cable drive, turning at the rate of $180 \mathrm{rpm}$, which could be used to stir the slurry. Because of space limitations, the equipment designed for use in the reactor could not be used inside the gamma facility.

The device for holding the slurry vessel assembly durIng the irradiation is shown in Figure 15. The flexible cable coupled to the 5 to 1 speed changer gave a stirring rate of $900 \mathrm{rpm}$. No provisions were made for purging the space above the vessel, because the gas collection system could be operated at atmospheric pressure. The rest of the 


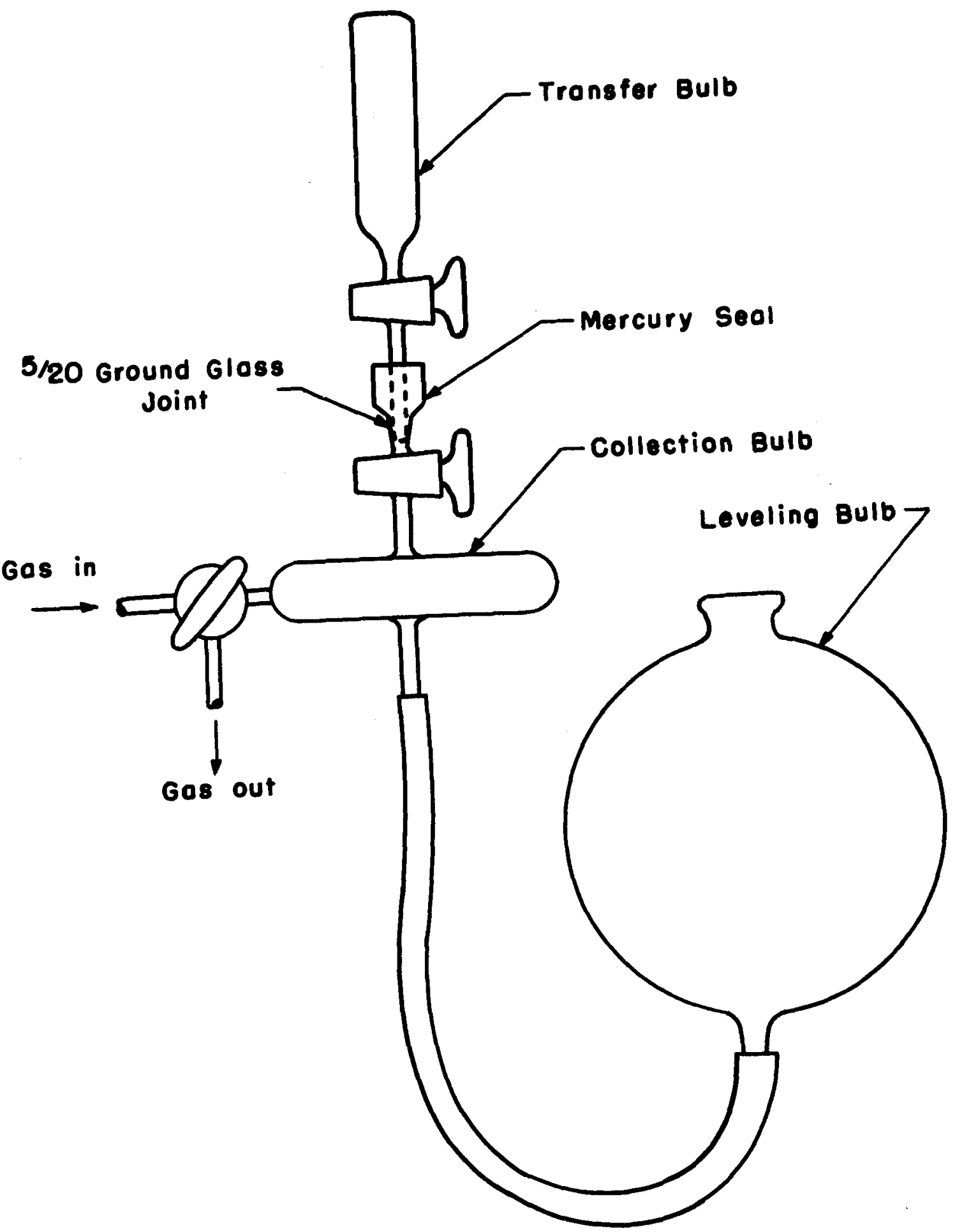

Figure 14. Gas sompling opporotus. 


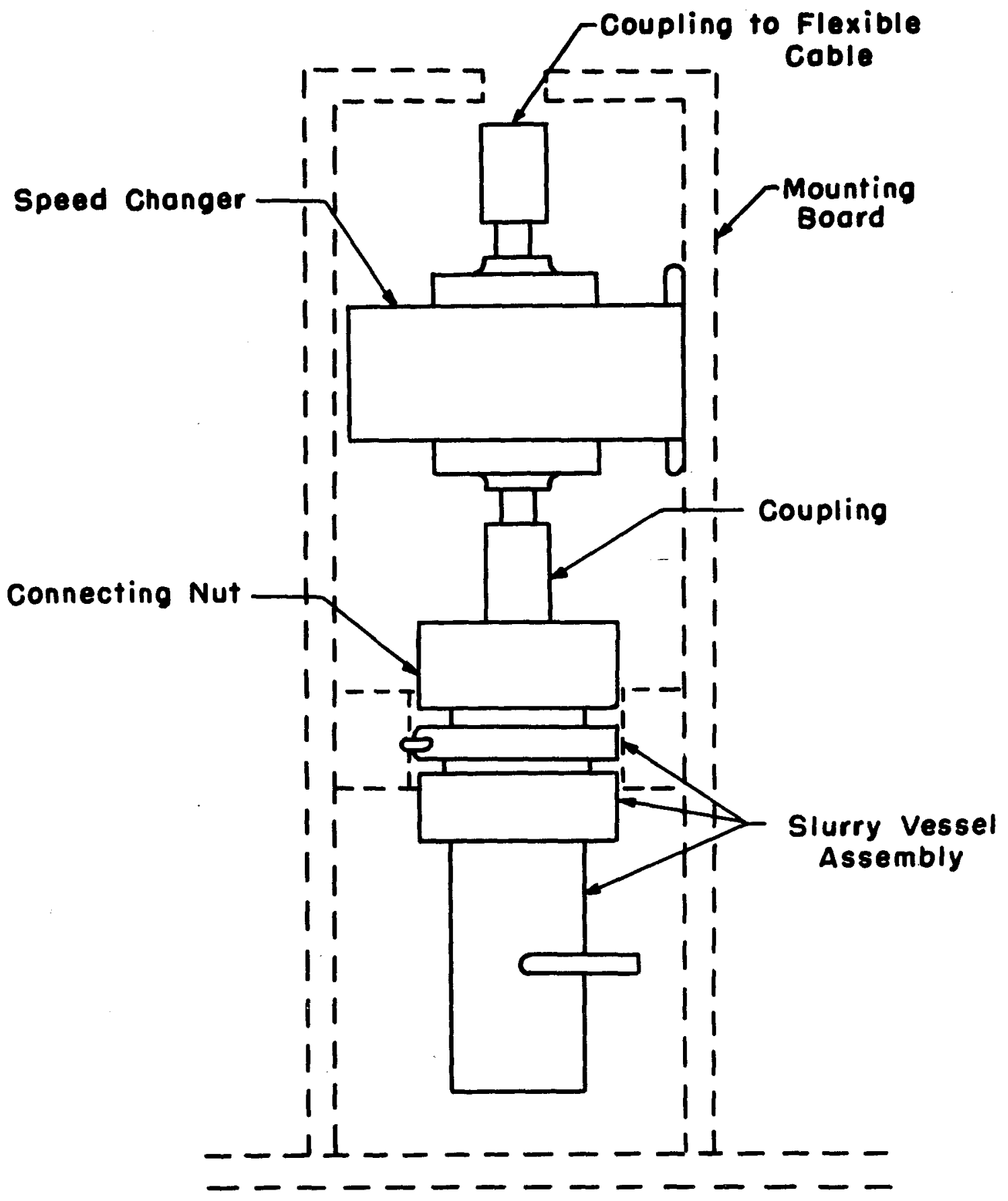

Figure 15. Arrangement of apparatus used with gamma source. 
system was the same as that discussed previously. Sample preparation

The fuel material used in this experiment was obtained as a product of a research project for the preparation of high-density, spherical thorlum oxide particles containing uranium (48). This fuel had been made by adding ammonium hydroxide to a solution of thorium nitrate to give a nitratestabilized sol which was adjusted nearly to the gel point. Then uranium nitrate was dissolved in the sol. When this solution was sprayed into an ammonia-air mixture, which caused the sol to gel, soft spherical particles were formed. These particles with uranium uniformly distributed throughout were then collected, washed, dried, and fired at $1,500^{\circ} \mathrm{C}$. The fuel used in this experiment contained 9.38 per cent uranium, had a density of about $9.75 \mathrm{gm} / \mathrm{cc}$, and varied from 5 to 75 microns in diameter.

This crude material had to be separated into particular size groups so that the effect of particle size could be examined. The first method of classifying this material, that of elutriation (49), did not give as fine a separation as was desired. By using a second method, that of repeated decanting (50), the desired separation was obtained. As applied here, this operation consisted of stirring some solid in a beaker of water containing about 0.1 per cent Calgon and then allowing the solid to settle. The principle of Stoke's law, which relates particle size to 
settling time, was used. When all of the particles in the size range desired had settled to the bottom, that which was still in suspension was poured out. This process was repeated until very little solid was left in suspension. The solid left in the beaker was then treated similarily, except the suspension was decanted as soon as all the particles greater than the size desired had settled. In this manner the crude material was separated into particular size groups. Figure 16 is a photomicrograph of the large-sized group.

Particle size measurement

Although the sample had been separated into particular size groups, 1t was still necessary to measure the particle size distribution within the group. Once this was known, it was weighted by the fraction of fission energy which escapes from a particle of that size. This gave the average fraction of energy escaping; the diameter corresponding to this average was that which was used in the experimental correlation.

The method chosen for measuring the particle size distribution was that of measuring the sedimentation rate by activation analysis $(51-53)$. Since the size distribution could have changed because of the stirring during the irradiation, it was decided to measure the size after the mun. Activity from the protactinium served as a tracer for the method. The rate of sedimentation was measured by counting 


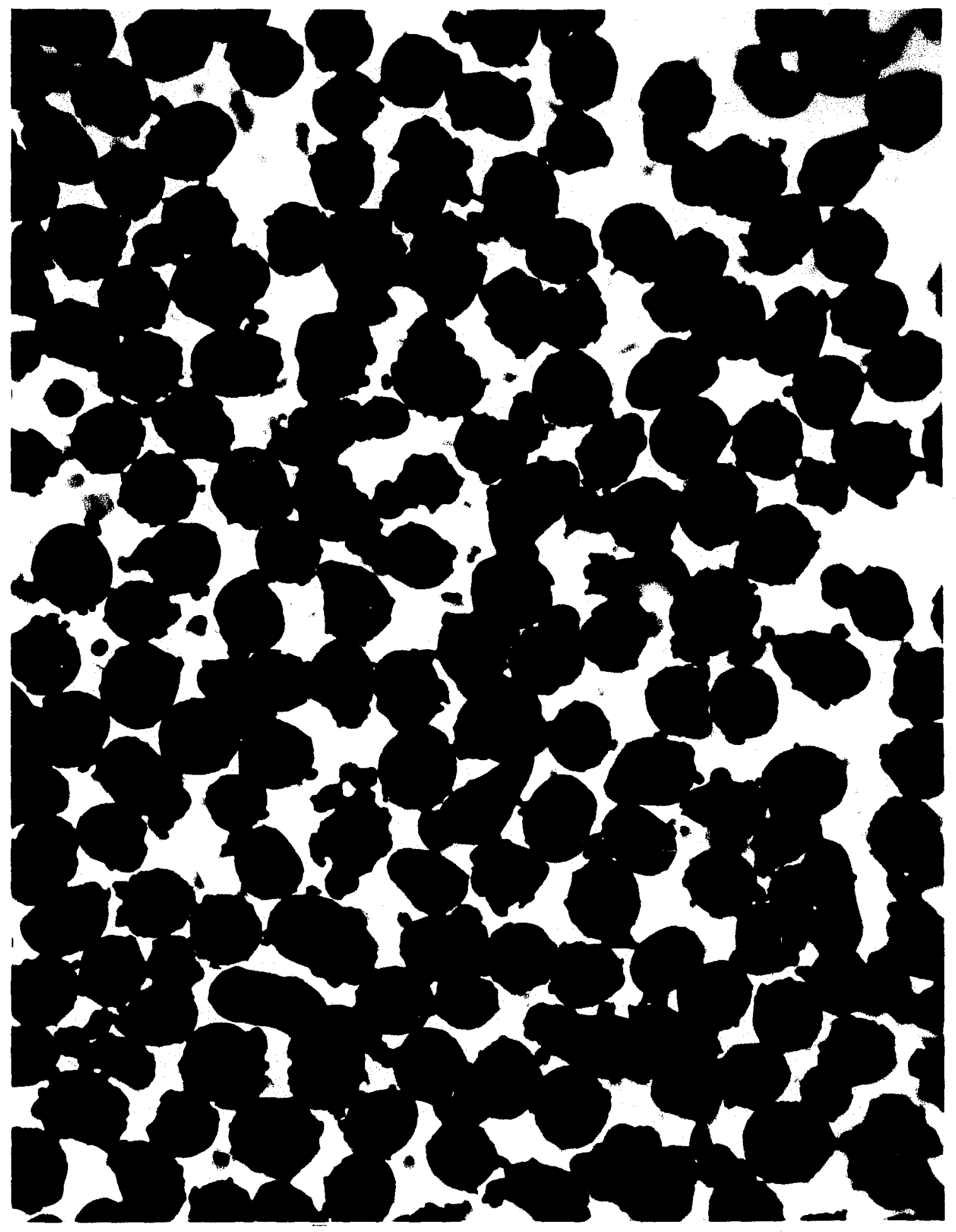

Figure 16. Photomicrograph of Urarija-Thoria Particles Which Are About 50 Microns in Diameter 
the activity of the particles as they passed by a slit in the shielding around a scintillation counter. From Stoke's law, the velocity of fall should be proportional to the square of the particle diameter.

Figure 17 shows a schematic drawing of the equipment used in this experiment. The chief components were the sedimentation column and the gamma counter, which was completely shielded except for a silt $1 / 8$ inch high by 1 inch wide. Height of liquid in the 1 in. I.D. column was 25 centimeters above the fritted disk and 10 centimeters above the level of the slit. The column was jacketed so that water from a constant temperature bath could be circulated around the column during the settling period. In order to suspend the solid in the liquid, air under 5 pounds per square inch was passed through the fritted filter. When the air was then shut off, the counter was started, and the integrated count was recorded as a function of time.

In order to use the relationship between settling rate and particle size, density and viscosity of the fluid and the density of the solid must be known. Several mixtures of glycerol and water were used as the fluid so that the particles would settle in a convenient time, which was also slow enough that Stoke's relationship was obeyed. From the density of the fluid, measured in a specific gravity bottle, the viscosity of the fluid was obtained from the Chemical 
SEDIMENTATION APPARATUS

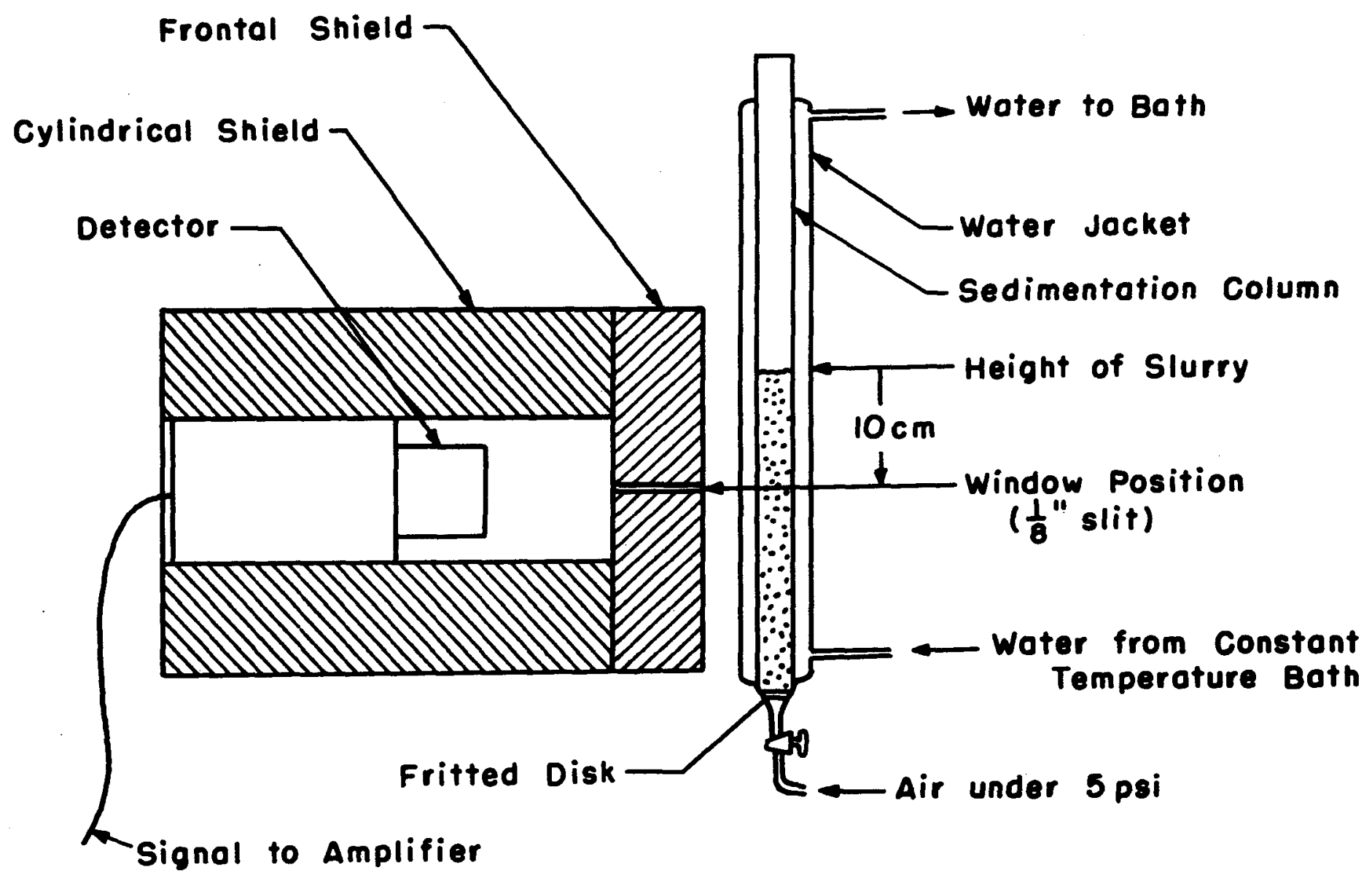

Figure 17. Diagram of the sedimentation apparatus. 
Rubber Handbook $(54,55)$. Density of the solid was also measured in the presence of the liquid in the same bottle. Gas analysis

Both hydrogen and oxygen are formed during the irradiation of water. Since hydrogen is a direct product of radiation, whereas oxygen comes from the decomposition of hydrogen peroxide, measurement of the hydrogen formed should be a better Indication of the water decomposition than that of oxygen. Moreover, hydrogen is not present in air, but oxygen could leak into the gas collection system. The choice of analytical technique was therefore dictated by the hydrogen rather than the oxygen.

The method which was chosen was gas chromatography (56). In this" application, argon was used as the carrier gas to sweep the gas sample, containing hydrogen, oxygen, and nitrogen, through a $1 / 4 \mathrm{in.} \mathrm{diameter} \mathrm{by} 5 \mathrm{ft}$. long column of copper tubing packed with size $13 \times$ molecular sieves from $F$ and $M$ Scientific Corporation. After the gases were separated in the column they passed through a thermal conductivity cell (katharometer). In this type of detector the sensitivity for hydrogen is a maximum when argon is used as the carrier gas. The signal from the chromatograph, a W11kens Instrument and Research, Inc. Model A-90-C Aerograph, was fed to a Honeywell Model 2HLA-7 amplifier and then to a Brown recorder Model SY153X18(VA)-X-118. 
The quantity of gas to be analyzed was measured in a Thomas-Van Slyke Manometric Apparatus manufactured by the Arther H. Thomas Co. Such a manometer was designed to measure the amount of gas in 11quids $(57,58)$, but it can be easily used to measure quantities of gas directly.

To calibrate the equipment, a blend of hydrogen and nitrogen was made up on the vacuum 11ne. Whenever unknown samples were to be analyzed, samples of this known blend were also analyzed. In this way the area of the peak for the unknown was always compared to that of the known, thereby eliminating the effect of possible day-to-day changes in the sensitivity.

Energy measurement

The amount of energy deposited in the slurry had to be determined in some manner because that was the basis for correlating the results. The method chosen was that of determining the number of fissions that occurred in the slurry by counting the amount of molybdenum-99 present in both liquid and solid phases. Knowing this quantity and the decay time, the number of fissions that must have occurred was calculated. The analytical procedure was a standard one at this laboratory; expected deviation was less than 5 per cent.

Calorimetric measurements have been used to give the total amount of energy absorbed during irradiation of 
uranium solutions (5). With the equipment used in this experiment, calorimetric measurements could not have been taken during the same irradiation that gas was collected. Correlation would have had to be made with the neutron flux measured by foll techniques. Even then there would still be the problem of determining the amount of energy that was due to the fissions. Measurement of the neutron flux with foils would not be a direct method elther. To accurately indicate the number of fissions which occurred in the slurry, the foll method must be calibrated with some other type of measurement.

Actually, foils were used to monitor the neutron flux and thereby served as a check on the energy measurement. Since gold folls would have become much too active at the dose received by the slurry, chromium in the form of its nitrate was suggested as an alternative (59). A few milligrams of chromium nitrate wrapped in aluminum foil was taped to the front of the gear box before each irradiation. Because of the relatively long half-life of chromium-51, 27.8 days (60), counting the activity could be conveniently delayed until all the runs were completed. Design of experiment

The experiment program was set up to study the effects of particle size and concentration of the slurry on the rate of water decomposition. Four different particle size groups were selected--three of them allowed about twice the energy 
to escape the parent particle than the next larger size; the fourth group included all those particles smaller than the rest. The cholce of solid concentration in the slurry was not an independent one. Enough fission energy had to escape the solid so that the effect of fission energy would be significantly greater than the effect of background radiation. The upper limit to the concentration was chosen with respect to a safe working level of the fission product radiation outside the reactor. As a result the particle sizes could neither be compared at the same levels of effective energy release nor at the same levels of density. Actual choice of the concentration levels was therefore somewhat arbitrary, but each particle size was compared at two levels of concentration.

In addition to these runs with the slurry, some experiments were needed to check out the equipment in the reactor and measure the amount of decomposition due to the background radiation. By irradiating a solution of potassium lodide, equations (15-17), the hydrogen rate due to the background radiation was measured. The irradiation of pure water was a check on the equipment. Since the hydrogen rate due to fission energy in solutions had been measured (5), the irradiation of solutions of uranium served as a further check on the equipment.

There were several relatively large sources of error in this experiment. The error in measuring the hydrogen 
flow rate could be estimated from the deviations in the flow rate during the run and from the deviations in hydrogen content of the samples collected during the run. Error in the energy input could be estimated from the combination of the results of the flux monitor and fission product analysis. Another source of error which could be large was that due to the recombination of the hydrogen in the presence of the high gamma background. An approximate estimate of this error could be obtained from the results of the runs with solutions of uranium, but a more reliable estimate could be obtained from the results of the slurry experiments themselves. If replicates were run, the error could have been estimated from the variance in the replit cates, but this would have doubled the number of experiments. It was decided then to neglect the effect of interaction between concentration and particle size in order to estimate this error.

Typical min

The run was started with the preparation of the sample. After the slurry vessel was first weighed to the nearest milligram, the desired amount of dry solid was added and the vessel rewelghed. Then the vessel, solid, and stirring assembly were welghed to the nearest tenth of a gram. After the approximate quantity of triply distilled water was ad-. ded, the unit was assembled and reweighed. Rubber policemen 
were then placed over the inlet and outlet tubes of the vessel so that none of the liquid could spill out.

Before the sample was ready to be used, 1 t was first irradiated with a dose of about $10^{6} \mathrm{r}$. in the co ${ }^{60}$ source chamber. Experlence had shown that this dose, about an hour's irradiation, was necessary to get rid of impurities. The slurry assembly was taken in 1ts transfer pot to the CP-5 reactor. This pot consisted of a 6 inch length of 4 inch diameter stainless steel pipe closed at the bottom and fitted with a flange at the top. The cover for the top was a $1 / 4$ inch thick brass plate. On the inside of the pot, there were two support arms in which the aluminum rods in the slurry assembly could rest. Polystyrene packing protected the vessel from damage during transit. Such precautions were necessary because the induced activity in the assembly from its previous irradiation would be about $100 \mathrm{mr} / \mathrm{hr}$.

Before the assembly was fastened to the rest of the equipment, the motor was turned on to make sure that the stirring mechanism was working. When the assembly had been attached and the tubes connected, the motor was again turned on to make sure that the stirring rod was engaged and the slurry suspended. Then the foll containing the chromium nitrate, which had been previously prepared, was taped to the front of the gear box. The slurry vessel was 
also taped to the gear box to prevent it from slipping off its "O" ring seal. The gas collection flasks were turned over so that the top flask was full of water. If there were no leaks when the valves to the collection flasks were opened, nitrogen flow was started by turning on the main cylinder valve and adjusting the flow rates on both the stripping and purging lines. Gas was allowed to flow through the system for several hours.

The equipment was then made ready for the irradiation. The pressure in the gas Iine was measured by shutting off the flow and reading the manometer. Barometric pressure and room temperature were also recorded. The flssion product trap was filled with liquid nitrogen. Flow through the soap-film flow meter was timed with a stopwatch and adjusted, if necessary. The rheostat was turned on, motor speed gradually increased, stirring checked, and then a plastic bag placed over the end of the irradiation assembly. If everything appeared to be running smoothly, the shielding blocks were removed from the reactor thermal column, and the beam catcher was moved up to the face of the reactor. Much of the gas collection equipment had been mounted on a mobile table so that the length of tubing between the beam catcher and equipment could be short; this table was then moved forward into position. Figure 18 shows a picture of the equipment as it would appear at this phase of the run. 
Top View

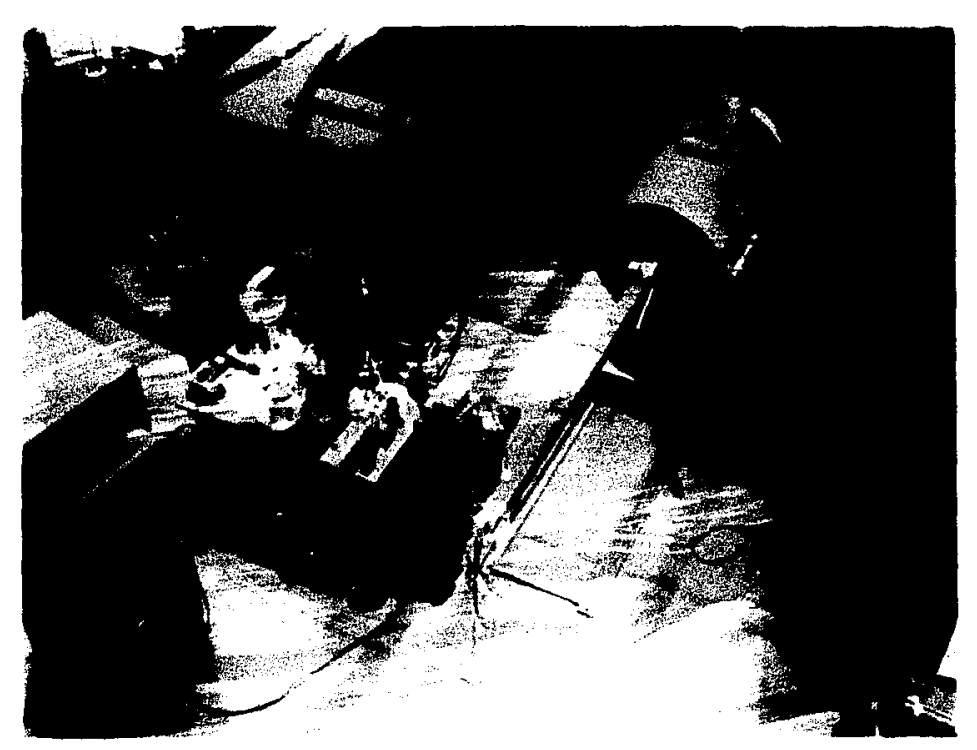

Front View

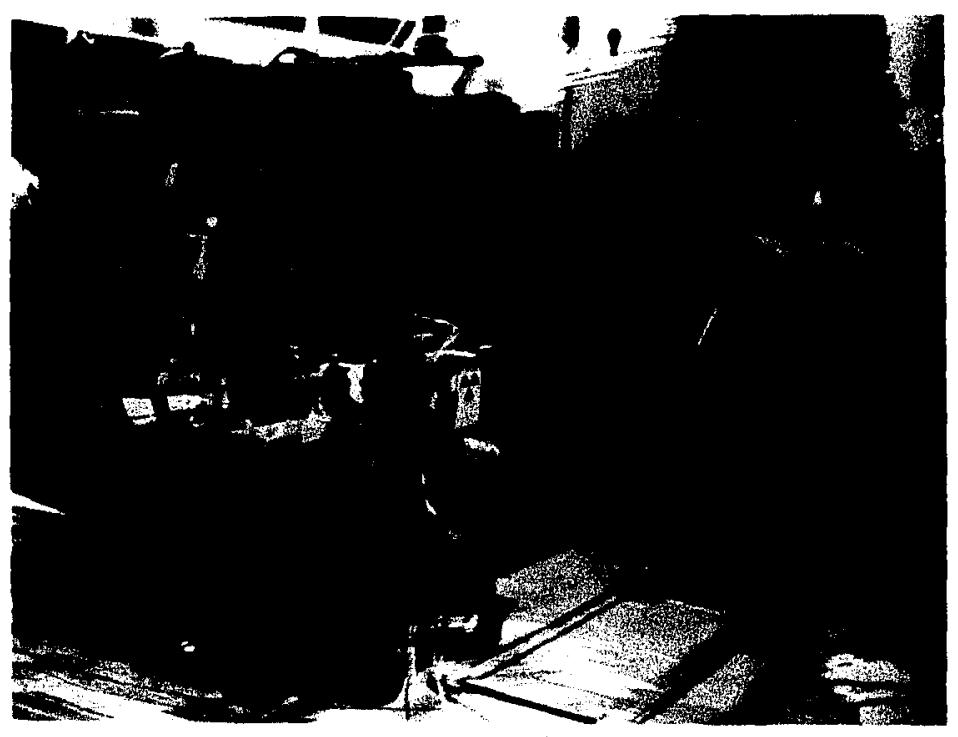

Figure 18. Photographs Showing Position of the Equipment Prior to the Start of an Irradiation 
After the thermal column gate had been opened four Inches, the cant1lever beam was cranked rapldly into the reactor. Start of the irradiation began when the stirring vessel reached its farthermost position. Usually it took less than ten seconds to crank the vessel into the reactor. The counter for the fission product monitor was turned on. Between one and two minutes after the start of the run the count rate began to increase rapidly. Periodically during the first twenty minutes of the run, the flow rate was timed and the total counts recorded. The flow was then diverted to the sample collector until about five milliliters of gas were collected. This quantity was transferred to an evacuated bulb. Two more samples were collected in the remaining ten minutes of the run, after which time the beam was cranked out and the gate closed. The stirring motor was then shut off, but gas flow was continued for several hours. Flow in the stripping line automatically stopped when the top gas collection flask was full and line pressure reached atmospheric.

About eight hours after the end of the mun, the beam catcher was moved back from the face of the reactor. Check of the equipment for radioactivity at this time usually showed that the slurry end of the stirring apparatus gave off about $5 \mathrm{r} / \mathrm{hr}$. Lead gloves were worn when the rubber connecting tubes were cut, the vessel assembly was unscrewed, 
mubber policemen were placed over the tubes, and the assembly was placed in the transfer pot.

Before the. slurry was removed from the vessel, the assembly was rewelghed to determine the loss of water. After the stirring assembly was separated from the quartz vessel, the slurry was scrapped out into a small bottle. What was then left in the vessel was washed out into a waste container. The vessel was now ready to be used again. The bottle containing the irradiated slurry sample was taken to the analytical group, where the solid and liquid were separated. Samples of both solid and liquid were analyzed for their molybdenum-99 content.

Meanwhlle the gas samples collected had to be analyzed. These were usually not run until at least a day after the irradiation, because of their radioactivity. The liquid nitrogen trap condensed out over 90 per cent of the activity; the rest was allowed to decay until there was less than an $\mathrm{mr} / \mathrm{hr}$ from a sample. Operating conditions on the chromatograph were as follows: argon flow rate $=60 \mathrm{cc} / \mathrm{min}$, filiment current $=140 \mathrm{ma}$, column temperature $=25^{\circ} \mathrm{C}$, and a chart speed $=4 \mathrm{in} / \mathrm{min}$. After recording the pressure on a $0.5 \mathrm{cc}$. sample of the 0.532 per cent hydrogen blend, the sample was forced up into a U-tube. By turning a four-way stopcock, one allowed the sample to be swept into the column. Approximately one minute later, the hydrogen peak 
appeared on the chart; after two minutes, oxygen; and before four minutes, nitrogen. This procedure was repeated for several samples of the known and then for 2.0 cc. samples of the unknown. Two samples from each of the collection bulbs were mun through the chromatograph.

The areas under the peaks were measured with $a \mathrm{~K}$ and E Compensating Polar Planimeter Model 4236M. Each of the hydrogen peaks was measured twice; oxygen peak area was also measured, but nitrogen was not. Since the area represented the volume of that particular gas in the sample, the composition of the sample was easily calculated.

Once the gas samples had been analyzed, the bulbs were reevacuated on a vacuum line equipped with a mercury diffusion pump. When the bulb had been evacuated, the stopcock was closed and mercury filled the leg of the bulb using a Töpler pump. After the bulb was removed from the line, the leg was capped to prevent any air from getting into it.

Several operations were necessary between experiments but were not done for each run. The Teflon packing in the stirring assembly and the Teflon bearing in the bottom of the stirring rod were replaced after about three irradiations; the "O" rings were replaced at the same time. The drying tubes in the gas collection system were refilled with indicating Drierite when necessary.

The foils containing the chromium.nitrate were analyzed after all the runs had been completed. Each foil was taped 
to the center of a card, then placed one centimeter from the detector, and counted for one minute.

Particle size analysis was delayed a month until all the runs were completed and the fission products analyzed. The sedimentation column was first filled to its liquid level mark with elther water or a mixture of glycerol and water. Into this volume of liquid, about $140 \mathrm{cc}$, was added a few milligrams of Calgon and about 4 grams of the irradiated solid. Air was then bubbled through the slurry until it was fully distributed--this took about an hour. At the same time that the air was shut off, the counter was turned on. The total count was recorded at time intervals during the setting until the liquid appeared clear and no change in count rate was observed. 
RESULTS

\section{$\underline{\text { Primary }}$}

The final result of these experiments is the value of $G\left(\mathrm{H}_{2}\right) \mathrm{f}$, the number of molecules of hydrogen produced for every $100 \mathrm{ev}$. of flssion energy deposited in the water. Each of the quantities used to obtain $G\left(\mathrm{H}_{2}\right)$ f is not directly measurable, but it is a result of a number of measurements. These results and the methods by which they were derived are given in this section.

All of the primary data recorded in this series of experiments is given in the Appendix. This includes the initial conditions of the mun, such as the weights of solid and liquid placed in the slurry vessel, room temperature and pressure, pressure of gas in the soap-film flow meter, and the reactor operating power. During the irradiation, flow rate, total counts, and the gas sampled were recorded as a function of the irradiation time. Analyses of the gas samples, as well as that of the calibration mixture, are also included in the Appendix. The number of fissions that occurred in the siurry was determined from the amount of molybdenum-99 found in the solid and liquid. An indication of the neutron flux was obtained from measurements of the activity induced in the chromium. Average particle size of the particles in the slurry was calculated from measurements of its sedimentation rate. 
Gas analyses

Experience with the gas chromatograph has shown that there is a tendency for the sensitivity of the instrument to drift with time. In order to account for this drift, samples of a known mixture of hydrogen and nitrogen were analyzed at the same time that unknown samples were analyzed. From a least squares treatment of the calibration mixture results, the sensitivity factor for hydrogen was found to be $0.0379 \mathrm{~mm} . \mathrm{H}_{2} / \mathrm{cm}^{2}$ with a standard deviation of 3.8 per cent for a $2.0 \mathrm{cc}$. sample during runs 4 through 8. For the rest of the muns, 9 through 21, this factor was 0.0423 with a standard deviation of 1.9 per cent.

The variance in the results of the calibration mixture could have been used to estimate the error in analyzing the unknowns, but that estimate was obtained from the replicates of the unknowns. In calculating the error variance, it was assumed that the standard deviation in the mean was proportional to the mean; because of scale factors, low concentrations can be measured as easily as high. The result was that the error variance, $\mathrm{s}_{e}{ }^{2}$, was equal to $1.28 \times 10^{-3}\left(\bar{x}_{2}\right)^{2}$, where $\bar{x}_{2}$ is the mean hydrogen content for a run. Variance in the calibration mixture was either equal to or less than this error variance, which indicates that drift was not a factor. 
An analysis of variance in the results of the hydrogen content of the gas samples is shown in Table 2. Such an analysis was necessary in order to determine the mean steady-state hydrogen content of the gas stream during the irradiation. The first five columns in the table give the customary sum of squares, degrees of freedom, mean hydrogen content, and the variance in that mean. The sixth column shows the ratio of the variance in the mean for each of the runs to the error varlance, which was calculated from all the replicates. The last column gives the E ratio for 99 per cent confidence with the degrees of freedom shown in the table. If the ratio of variances is greater than the 99 per cent confidence limit, the variation in the hydrogen content of the samples from that run was probably not due to any error in the gas analysis. However, the variation could be due to fluctuations in the gas flow rate to be described next.

Gas flow rate through the slurry

Examination of the flow rate, measured by timing the soap film in the flow meter with a stopwatch, is shown in Table 3. The number of measurements which were used to estimate the flow rate during the time that samples were collected is given in the second column. This does not necessarily represent the total number of measurements taken during the mun. The mean flow rate was obtained by 
TABLE 2

ANALYSIS OF VARIANCE IN GAS SAMPLING

\begin{tabular}{|c|c|c|c|c|c|c|}
\hline Run & $\begin{array}{l}\text { Sum of } \\
\text { Squares }\end{array}$ & $\begin{array}{l}\text { Degrees of } \\
\text { Freedom }\end{array}$ & $\mathrm{H}_{2}$ mean, $\overline{\mathrm{x}}_{2}$ & Variance, $s_{2}{ }^{2}$ & $F, s_{2}^{2} / s_{\epsilon}{ }^{2}$ & $\mathrm{~F}, 99 \%$ \\
\hline 4 & $0.12 \times 10^{-3}$ & 4 & $0.259 \times 10^{-2}$ & $30 \times 10^{-6}$ & 0.30 & 3.7 \\
\hline 5 & 0.259 & 2 & 0.131 & 295 & $13 \cdot 5$ & 5.1 \\
\hline $6^{a}$ & 0.081 & 1 & 0.212 & 81 & 1.4 & $7 \cdot 3$ \\
\hline 7 & 0.0022 & 1 & 0.0156 & 2.2 & 7.0 & $7 \cdot 3$ \\
\hline 8 & 0.0023 & 2 & 0.0612 & 1.2 & 2.5 & 5.1 \\
\hline 9 & 27.4 & 2 & 0.0817 & 18,700 & 2,200 & 5.1 \\
\hline 10 & 0.799 & 2 & 0.0909 & 400 & 38 & 5.1 \\
\hline 11 & 1.01 & 2 & 0.157 & 500 & 16 & 5.1 \\
\hline 12 & 990 & 3 & 0.541 & 330,000 & 880 & 4.3 \\
\hline 13 & 0.17 & 2 & 0.146 & 80 & 2.9 & 5.1 \\
\hline 14 & 0.128 & 2 & 0.429 & 64 & 0.27 & 5.1 \\
\hline 15 & 9.6 & 2 & 0.157 & 4,800 & 150 & 5.1 \\
\hline 16 & 0.018 & 2 & 0.192 & 9.0 & 0.2 & 5.1 \\
\hline 17 & 0.65 & 2 & 0.279 & 330 & 3.4 & 5.1 \\
\hline 18 & 0.175 & 2 & 0.178 & 88 & 2.2 & 5.1 \\
\hline $19^{\mathrm{b}}$ & 1.50 & 1 & 0.226 & 1,500 & 22 & $7 \cdot 3$ \\
\hline 20 & 1.21 & 2 & 0.692 & 620 & 1.0 & 5.1 \\
\hline 21 & 0.0057 & 3 & 0.0156 & 1.9 & .8 & 4.3 \\
\hline
\end{tabular}


TABLE 3

GAS FLOW RATE

\begin{tabular}{|c|c|c|c|c|c|c|c|c|}
\hline \multirow{2}{*}{\multicolumn{2}{|c|}{\begin{tabular}{c|c|} 
Run & $\begin{array}{c}\text { Measure- } \\
\text { ments }\end{array}$
\end{tabular}}} & \multirow{2}{*}{\multicolumn{2}{|c|}{$\begin{array}{l}\text { Measured Flow Rate } \\
\text { Mean,cc/sec }\end{array}$}} & \multirow{2}{*}{${ }^{\mathrm{T}} \mathrm{C}$} & \multicolumn{2}{|c|}{ Pressure $\mathrm{mm}$. $\mathrm{Hg}$} & \multicolumn{2}{|l|}{ Flow Rate, STP } \\
\hline & & & & & & & Wean, $x_{1}$, ce/min & $\cos 101160,1^{2}$ \\
\hline 4 & 18 & 0.171 & $0.13 \times 10^{-4}$ & 26.3 & 746.7 & 25.7 & 8.87 & 0.035 \\
\hline 5 & 15 & 0.173 & 2.4 & 26.6 & 747.1 & 26.1 & 8.95 & 0.642 \\
\hline 6 & 10 & 0.258 & 0.36 & 26.8 & 740.3 & 26.4 & $13 \cdot 2^{a}$ & 0.094 \\
\hline 7 & 19 & 1.42 & 15.8 & 27.0 & 741.8 & 26.7 & 72.7 & 4.16 \\
\hline 8 & 20 & 0.256 & 1.22 & 25.8 & 740.0 & 24.9 & $13 \cdot 3$ & 0.327 \\
\hline 9 & 17 & 0.260 & 0.174 & 25.2 & 735.0 & 24.0 & 13.4 & 0.046 \\
\hline 10 & 11 & 0.268 & 0.18 & 26.8 & 733.4 & 26.4 & 13.6 & 0.047 \\
\hline 11 & 16 & 0.302 & 3.50 & 26.0 & 743.0 & 25.2 & 15.6 & 0.93 \\
\hline 12 & 27 & 0.266 & 2.03 & 26.0 & 738.0 & 25.2 & 13.6 & 0.534 \\
\hline 13 & 20 & 0.438 & 0.556 & 26.0 & 733.0 & 25.2 & $22 \cdot 3$ & 0.145 \\
\hline 14 & 18 & 0.274 & 0.589 & 27.5 & 735.3 & $27 \cdot 5$ & 13.9 & 0.152 \\
\hline 15 & 14 & 0.284 & 0.368 & 27.1 & 739.6 & 26.9 & 14.5 & 0.097 \\
\hline 16 & 19 & 0.318 & 1.29 & $27 \cdot 7$ & 739.4 & 27.8 & 16.2 & 0.334 \\
\hline 17 & 19 & 0.305 & 0.633 & 28.0 & 734.8 & $28 \cdot 3$ & 15.4 & 0.161 \\
\hline 18 & 15 & 0.311 & 2.01 & $27 \cdot 3$ & 732.5 & 27.2 & 15.7 & 0.514 \\
\hline 19 & 17 & 0.488 & 11.7 & 28.0 & 734.9 & $28 \cdot 3$ & $24.7^{b}$ & 3.00 \\
\hline 20 & 13 & 0.280 & 0.534 & 27.1 & 725.2 & 26.9 & 14.1 & 0.135 \\
\hline 21 & $\frac{22}{2 \pi}$ & 0.232 & 3.81 & 27.7 & 727.4 & 27.8 & 11.6 & 0.96 \\
\hline
\end{tabular}


averaging the reciprocal of the rate which was measured in units of seconds per 10 cubic centimeters. The variance in this mean is given in the fourth column. In order to know the flow rate at standard conditions, corrections had to be made for temperature and pressure. Since the gas was measured over a soap solution, 1t was assumed to be saturated with water vapor. Even if the gas were dry, the error due to this assumption would be less than 4 per cent. The last two columns give the mean and its varlance of the flow rate as standard conditions. Note that on two of the mus the flow rate given is restricted to the gas samples indicated because the flow rate was changing during the experiment.

Since the variation in the hydrogen content of the samples taken during a run could be a result of fluctuations in the gas flow rate, an analysis of variance in the hydrogen flow rate, shown in Table 4, was made to check this possibility. In order to compare the variance in the mean hydrogen content to that in the mean flow rate, the value of the mean in both cases must be the same. A simple way of satisfying this condition was to correct the mean to unity by dividing the varlance by the square of the mean. The sixth column gives the ratio of variances--that of the hydrogen content to the sum of that of the flow rate and that of analysis. The last column gives the 99 per cent confidence limits for the "F" ratio with the degrees of 
TABLE 4

ANALYSIS OF VARIANCE FOR HYDROGEN RATES

\begin{tabular}{|c|c|c|c|c|c|c|}
\hline \multirow[t]{2}{*}{ Run } & \multirow{2}{*}{$\begin{array}{l}\text { Flow Rate } \\
\mathrm{s}_{1}{ }^{2} / \overline{\mathrm{x}}_{1}{ }^{2}\end{array}$} & \multirow{2}{*}{$\begin{array}{l}\text { Hydrogen } \\
\mathrm{s}_{2}{ }^{2} / \bar{x}_{2}{ }^{2}\end{array}$} & \multicolumn{2}{|c|}{ Degrees of Freedom } & \multicolumn{2}{|l|}{$\mathrm{s}_{2}{ }^{2} / \bar{x}_{2}{ }^{2}$} \\
\hline & & & in $s_{2}$ & in $s_{1}$ & $F, s_{1} 2 \bar{x}_{1}^{2}+s_{e}^{2} / \bar{x}_{2}^{2}$ & F, 99\% \\
\hline 4 & $0.445 \times 10^{-3}$ & $0.386 \times 10^{-3}$ & 4 & 18 & 0.22 & 4.6 \\
\hline 5 & 6.44 & 17.2 & 2 & 15 & 2.2 & 6.3 \\
\hline 6 & 0.55 & 1.79 & 1 & 10 & 1.0 & 10 \\
\hline 7 & 0.79 & 9.0 & 1 & 19 & $4 \cdot 3$ & 8.2 \\
\hline 8 & 1.85 & 3.27 & 2 & 20 & 1.0 & 5.8 \\
\hline 9 & 0.26 & 2,820 & 2 & 17 & 1,800 & 6.1 \\
\hline 10 & 0.25 & 48.4 & 2 & 11 & 32 & $7 \cdot 2$ \\
\hline 11 & 3.8 & 20.4 & 2 & 16 & 4.1 & 6.2 \\
\hline 12 & 2.9 & 1,120 & 3 & 27 & 270 & 4.6 \\
\hline 13 & 0.19 & $3 \cdot 78$ & 2 & 20 & 2.6 & 5.8 \\
\hline 14 & 0.79 & 0.35 & 2 & 18 & 0.17 & 6.0 \\
\hline 15 & 0.46 & 191 & 2 & 14 & 110 & 6.5 \\
\hline 16 & 1.27 & 0.26 & 2 & 19 & 0.10 & 5.9 \\
\hline 17 & 0.68 & $4 \cdot 3$ & 2 & 19 & 2.2 & 5.9 \\
\hline 18 & 2.09 & 2.76 & 2 & 15 & 0.82 & 6.4 \\
\hline 19 & 4.91 & 28 & 1 & 17 & 4.5 & 8.4 \\
\hline 20 & 0.68 & 1.3 & 2 & 13 & 0.66 & 6.7 \\
\hline 21 & 7.1 & 8 & 3 & 21 & 0.95 & 4.9 \\
\hline
\end{tabular}


freedom shown in the table. Examination of the figures in the last two columns shows that the varlation in the mean hydrogen content for runs 9, 10, 12, and 15 was not due to fluctuations in the flow rate.

The varlation in hydrogen content for those four runs can be explained, for after each of those experiments it was observed that the stirring mechanism was not working. Since mun 8 , containing a solution of uranium, was mun without stirring, this peculiar behavior was limited to slurries. Hydrogen gas probably accumulated in the settled slurry and escaped in surges. Total fission energy

Estimation of the amount of fission energy released during the irradiation was based on the analysis of the fission product molybdenum-99. The analyst was easily able to determine the number of fissions in the uranium solutions, but, due to lack of experience, he had difficulty in dissolving the solid phase of the slurry. As a result, all the solid samples were analyzed at the same time after a suitable dissolving technique was worked out. Since this fission product has a half-life of only 67 hours, the activity was somewhat less for the earlier runs than for the later. The presence of impurities, which would tend to increase the fission yield, would have a larger effect on these earlier runs. Such an effect is pointed out in the third column of 
Table 5. After mun 8 it was noticed that the irradiation vessel was not as far into the reactor for muns through 7 as it was for the rest of the muns. For that reason the fission yields for runs 5 and 6 are less than that for mun 8. However, the yields for the slurry runs should only be about 5 per cent higher than mun 8 because of the increase in reactor power. For the last six runs (15-20) this condition is satisfied.

In order to obtain a better estimate of the fission yield, the fission yield in the third column was divided by the flux monitor $\left(\mathrm{Cr}^{51}\right)$ in the fourth column to give the monitor factor shown in the fifth column. Since the monitor factor is a ratio of two estimates of the neutron flux, it should be a constant for all the muns. For the more consistent runs 15 to 20 , the mean value of this factor is $2.18 \times 10^{10}$ with a standard deviation of $0.15 \times 10^{10}$. The mean value of the monitor factor was then divided by the flux monitor values to give the corrected fission yield shown in the sixth column.

The total rate of fission energy released in the slurry was calculated from the corrected fission yield, using the factors of 9.38 per cent uranium in the solid and $165 \mathrm{Mev}$. per fission (15). The neutron flux can also be calculated from the fission yield; its value was about $1.8 \times 10^{12}$ neutrons $/ \mathrm{cm}^{2} / \mathrm{sec}$. Estimation of the flux from the chromium 
TABLE 5

TOTAL RATE OF FISSION ENERGY IN THE SLURRY

\begin{tabular}{|c|c|c|c|c|c|c|}
\hline Run & Fissions & $\begin{array}{c}\text { Fission Yield, } \\
\mathrm{f} / \mathrm{gm} \mathrm{U} / \mathrm{min}\end{array}$ & $\begin{array}{l}\text { Flux Monitor, } \\
\text { Counts/mg/min }\end{array}$ & $\begin{array}{l}\text { Monitor } \\
\text { Factor }\end{array}$ & $\begin{array}{l}\text { Corrected } \\
\text { Fission Yield, } \\
\mathrm{f} / \mathrm{gm} \mathrm{U/min}\end{array}$ & $\begin{array}{c}\text { Total Energy Input, } \\
\text { Mev/min/gm } \mathrm{H}_{2} \mathrm{O}\end{array}$ \\
\hline 5 . & $50.5 \times 10^{30}$ & $0.684 \times 10^{12}$ & $\cdot \cdot \cdot \cdot \cdot \cdot \cdot$ & . . . . . & $\cdot \cdot \cdot \cdot \cdot \cdot \cdot$ & $0.297 \times 10^{12 b}$ \\
\hline 6 & 237 & 0.711 & $! \cdot \cdot \cdot \cdot \cdot \cdot \cdot$ & $\cdot \ldots \cdot \cdot \cdot$ & $\cdot \cdot \cdot \cdot \cdot \cdot \cdot \cdot$ & $1.54^{b}$ \\
\hline 8 & 67.9 & 0.914 & !. $\cdot \cdot \cdot \cdot \cdot \cdot$ & - . - . & . $\cdot \cdot \cdot \cdot \cdot$ & $0.397^{b}$ \\
\hline 9 & 2,365 & 1.57 & 45.9 & $3.42 \times 10^{10}$ & $1.00 \times 10^{12}$ & 8.70 \\
\hline 10 & 2,060 & 1.37 & 47.1 & 2.91 & 1.03 & 8.83 \\
\hline 11 & 5,988 & 1.96 & 47.1 & 4.16 & 1.03 & 20.0 \\
\hline 12 & 7,020 & 1.21 & $47 \cdot 7$ & 2.54 & 1.04 & 42.6 \\
\hline 13 & 3,212 & 2.61 & 47.4 & 5.5 & 1.035 & 9.21 \\
\hline 14 & 4,810 & 1.97 & 47.1 & 4.2 & 1.03 & 20.6 \\
\hline 15 & 2,767 & 1.07 & $46.8^{a}$ & 2.29 & 1.03 & 20.5 \\
\hline 16 & 739 & 1.15 & 50.6 & 2.27 & 1.02 & 4.90 \\
\hline 17 & 741 & 1.03 & 47.9 & 2.15 & 1.10 & 6.34 \\
\hline 18 & 2,695 & 1.06 & 45.4 & $2 \cdot 33$ & 0.99 & 20.2 \\
\hline 19 & 1,225 & 0.87 & 45.1 & 1.93 & 0.98 & 9.08 \\
\hline 20 & 2,340 & 0.99 & $46.8^{a}$ & 2.12 & 1.02 & 20.1 \\
\hline
\end{tabular}

a Mean value

${ }^{b}$ Increased by a factor of 1.05 because reactor power had changed. 
activation gave a value of $2.5 \times 10^{12}$. The fact that the foll was closer to the reactor core than the slurry could be the reason for the higher neutron flux.

\section{Effective fission energy}

The fraction of fission energy which escaped to the liquid depended upon the particle size in the slurry and its concentration. The particle size used in each run was calculated from the data on the sedimentation rate by accumulatively multiplying the fraction of particles that had fallen out of the counting zone during a time interval by the particle diameter corresponding to that time. This result, a sample of which is given in Appendix III, 171, gave the average particle size. However, since energy release varied with diameter, it was necessary to weigh the particle size distribution with the fraction of energy that would escape to get the energy-average particle size. This was done in the same manner as above, except the fraction of energy escaping the solid was used in place of the diameter. Values for this fraction were obtained from the zero concentration curve in Figure 1, using an average range of 8 microns. The result of this calculation gave the fraction of energy that should escape from the solid for that particular size distribution. The diameter of a particle that would allow this same fraction to escape was taken as the energy-average particle size. Table 6 gives the results of these calculations. 
TABLE 6

PARTICLE SIZE ANALYSES - RESULTS

\begin{tabular}{|c|c|c|c|c|}
\hline Run & $\begin{array}{c}\text { Number-average } \\
\text { diameter, } \mu\end{array}$ & $\begin{array}{l}\text { Percentage } \\
\text { of energy } \\
\text { released }\end{array}$ & $\begin{array}{c}\text { Energy-average } \\
\text { diameter, } \mu\end{array}$ & $\begin{array}{c}\text { Size } \\
\text { used, } \mu\end{array}$ \\
\hline $11 \mathrm{~A}$ & 52.7 & 9.44 & $51 \cdot 5$ & \\
\hline $11 B$ & $51 \cdot 3$ & 9.34 & 52.0 & \\
\hline $18 \mathrm{~A}$ & 46.8 & 10.83 & 44.8 & 115 \\
\hline $18 \mathrm{~B}$ & 46.7 & 10.69 & 45.4 & \\
\hline $13 \mathrm{~A}$ & 17.2 & $28 \cdot 33$ & 16.6 & \\
\hline $14 \mathrm{~A}$ & 17.7 & 28.85 & 16.3 & 16 \\
\hline $14 \mathrm{~B}$ & 16.5 & 30.01 & 15.6 & \\
\hline $16-19 \mathrm{~A}$ & 7.99 & 54.04 & 7.87 & 70 \\
\hline $16-19 \mathrm{~B}$ & 7.89 & 54.41 & 7.77 & 1.0 \\
\hline $17-20 \mathrm{~A}$ & 6.43 & 61.73 & 6.31 & \\
\hline $17-20 \mathrm{~B}$ & 6.43 & 61.67 & 6.31 & ) \\
\hline
\end{tabular}


The effect of concentration may now be determined from the curves in Figure 1. The results are tabulated in Table 7 for those muns in which the hydrogen flow rate is known. The diameter to range ratios in the table were based on the range values of 8.8 microns and 7.0 microns for the light and heavy fission fragments, respectively. The fraction of the total energy that was deposited in the water was obtained from the energy-weighted average of the energy that escaped the light and heavy fragments. The original energy of the light fragment was taken as $100 \mathrm{Mev}$; that for the heavy, $65 \mathrm{Mev}$ (15). This information was used in determining hydrogen ylelds per $100 \mathrm{ev}$, or $G\left(\mathrm{H}_{2}\right) f^{\circ}$ 
TABLE 7

FRACTION OF FISSION ENERGY RELEASED TO THE WATER

\begin{tabular}{l|c|c|c|c|c|c|c|}
\hline \hline \multirow{2}{*}{ Run } & $\begin{array}{c}\text { Solid volume } \\
\text { fraction }\end{array}$ & $\begin{array}{c}\text { Particle } \\
\text { size, } \mu\end{array}$ & \multicolumn{2}{|c|}{ Diameter/range } & \multicolumn{2}{|c|}{ Energy fraction to water } \\
\hline 11 & 0.11 & 52 & 6.0 & Heavy & Light & Heavy & Total \\
13 & 0.054 & 16 & 1.9 & 2.4 & 0.10 & 0.08 & 0.092 \\
14 & 0.114 & 16 & 1.9 & 2.4 & 0.27 & 0.23 & 0.255 \\
16 & 0.030 & 7.8 & 0.91 & 1.15 & 0.54 & 0.47 & 0.513 \\
17 & 0.036 & 6.3 & 0.735 & 0.93 & 0.60 & 0.53 & 0.572 \\
18 & 0.116 & 45 & 5.25 & 6.6 & 0.11 & 0.09 & 0.102 \\
19 & 0.056 & 7.8 & 0.91 & 1.15 & 0.51 & 0.45 & 0.486 \\
20 & 0.112 & 6.3 & 0.735 & 0.93 & 0.53 & 0.47 & 0.506 \\
\hline
\end{tabular}




\section{DISCUSSION OF RESULTS}

Hydrogen yield

Now that the hydrogen flow rate and the fission energy released to the water are known, $\mathrm{G}\left(\mathrm{H}_{2}\right)_{f}$, the hydrogen yield due to fission energy, can be calculated. Since the hydrogen rate that has been measured is caused by both fission energy and background energy, the following method was chosen for separating these effects: Assume that the effects of the different radiations are additive; data in the I1terature (61) support this assumption. Represent the hydrogen rate in molecules per minute due to fissions by $d\left(\mathrm{H}_{2}\right)_{\mathrm{f}} / \mathrm{dt}$ and that due to the other energy by $\mathrm{d}\left(\mathrm{H}_{2}\right)_{\gamma} / \mathrm{dt}$. Then the total hydrogen rate, $d\left(H_{2}\right)_{t} / d t$, can be written as the sum of these two: $\mathrm{d}\left(\mathrm{H}_{2}\right)_{t} / \mathrm{dt}=\mathrm{d}\left(\mathrm{H}_{2}\right) \gamma / \mathrm{d} t+\mathrm{d}\left(\mathrm{H}_{2}\right)_{\mathrm{f}} / \mathrm{dt}$. As sume that the rate due to the background radiations is dependent only on the reactor power level and the weight of water in the sample. Then $\left(1 / \mathrm{P}_{0} \mathrm{~W}_{1}\right) \mathrm{d}\left(\mathrm{H}_{2}\right)_{\gamma} / \mathrm{dt}$ should be a constant when $\mathrm{P}_{0}$ is the ratio of the reactor power to 4.0 megawatts and $W_{1}$ is the Iiquid weight in the sample. Assume that the rate due to the fissions depends only on the amount of fission energy that escapes to the liquid. Then $\left(\mathrm{dt} / \mathrm{dE_{1 }}\right) \mathrm{d}\left(\mathrm{H}_{2}\right)_{\mathrm{f}} / \mathrm{dt}$, where $\mathrm{dE}_{1} / \mathrm{dt}$ is the rate that fission energy escapes to the Ilquid, should be a constant. In fact, when $d E_{1} / d t$ is expressed in units of hundred electron volts per minute, 
this constant becomes just $G\left(\mathrm{H}_{2}\right)_{f}$. A plot of the data in the form of the equation:

$$
\frac{d\left(\mathrm{H}_{2}\right)_{t}}{\left(\mathrm{P}_{\mathrm{O}} \mathrm{W}_{1}\right) d t}=\frac{\mathrm{d}\left(\mathrm{H}_{2}\right) \gamma}{\left(\mathrm{P}_{0} \mathrm{~W}_{1}\right) d t}+G\left(\mathrm{H}_{2}\right)_{f} \cdot \frac{d E_{1}}{\left(\mathrm{P}_{0} \mathrm{~W}_{1}\right) d t}
$$

should have a slope of $\mathrm{G}\left(\mathrm{H}_{2}\right)_{f}$ and intercept of $\left(1 / \mathrm{P}_{\mathrm{o}} \mathrm{W}_{1}\right) \mathrm{d}\left(\mathrm{H}_{2}\right)_{\gamma} / \mathrm{dt}$.

The hydrogen evolution rate and the effective energy deposition rate are given in the necessary form in Table 8. These values were then plotted on the graph in Figure 19. The box around each point in the figure represents the 10cation of the point with 90 per cent confidence; only those points for which this $11 \mathrm{mit}$ is within 20 per cent of their mean are shown on this graph. Slurry muns 11 and 19 are therefore not shown. Limits on the hydrogen rate were estimated from the variance in the hydrogen content of the gas samples, and those for the energy were estimated from the variance in the monitor factor. The only line that can be drawn through the limits of all the runs has a slope of 2.1 . The dotted Iines in the figure are those which can be drawn through all but one of the experimental points; these could represent the limiting values for $G\left(\mathrm{H}_{2}\right) f^{\circ}$ Analysis of variance

An analysis of variance in the G-values shown in Table 8 was made to determine the possible effects of particle size and concentration. Table 9 gives the results of this analysis. 
TABLE 8

HYDROGEN YIELDS

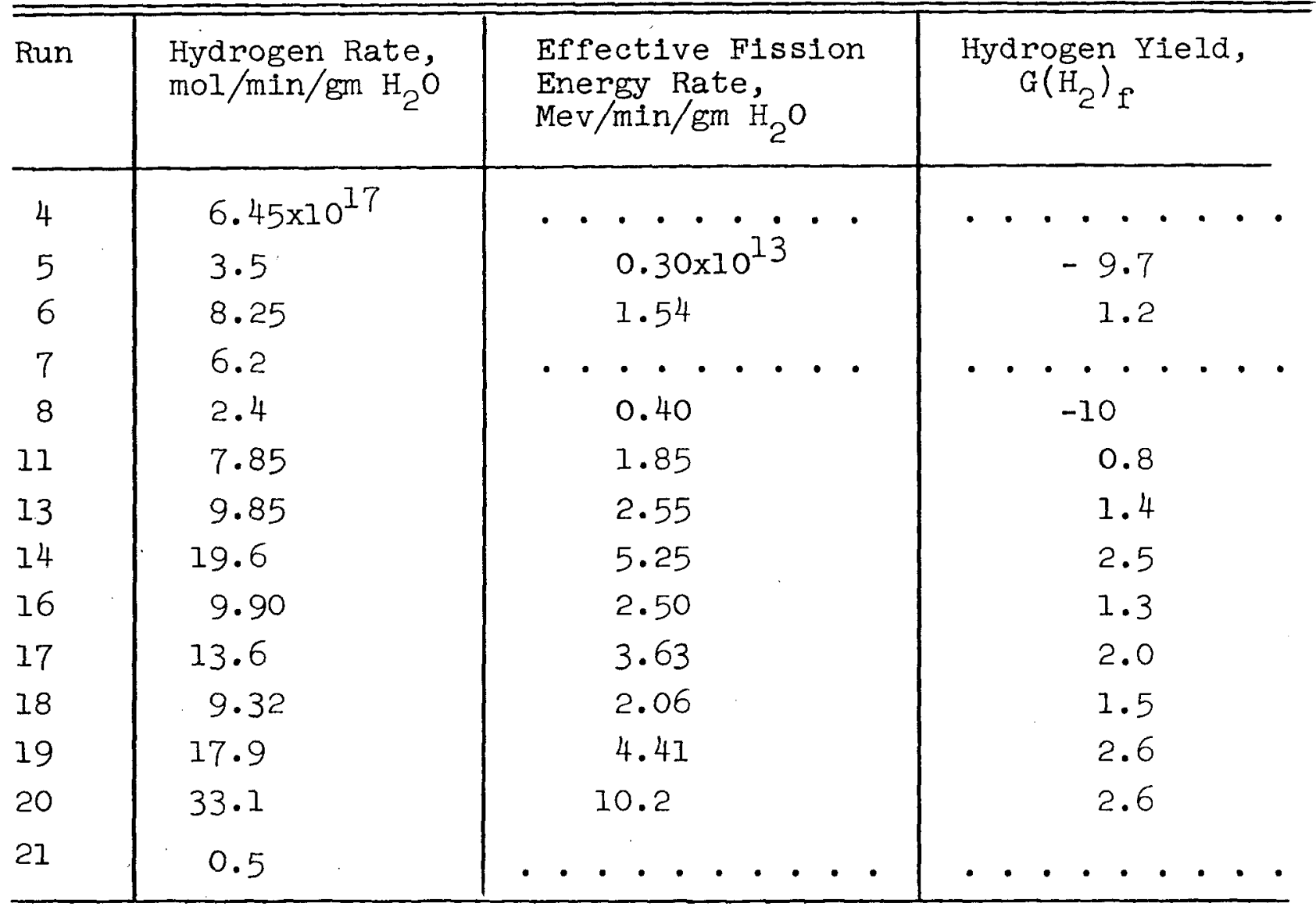




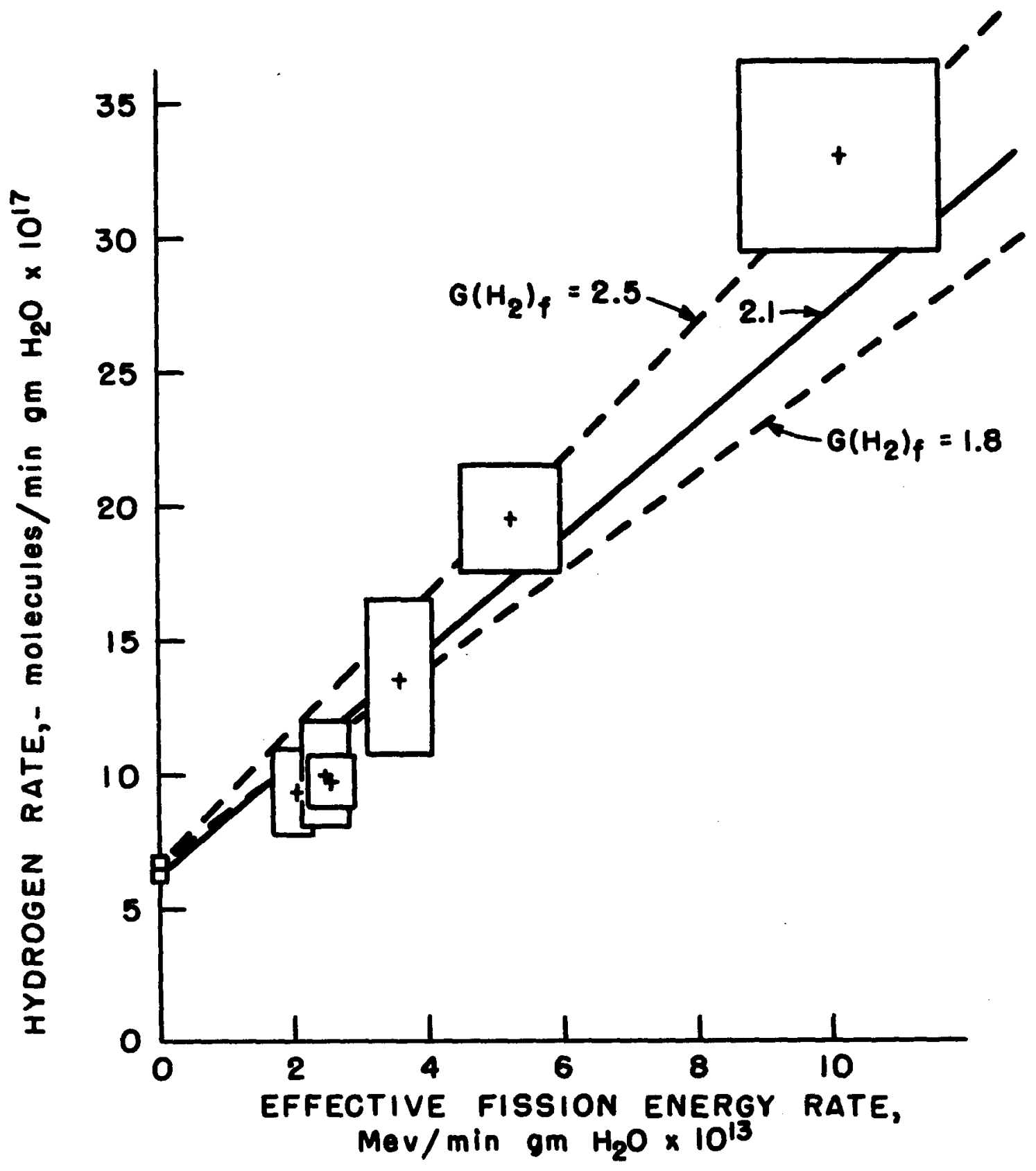

Figure 19. Hydrogen yield with experimental error. 
TABLE 9

ANALYSIS OF VARIANCE - EFFECTS OF PARTICLE SIZE AND CONCENTRATION

\begin{tabular}{|c|c|c|c|c|c|}
\hline $\begin{array}{l}\text { Source of } \\
\text { Variation }\end{array}$ & $\begin{array}{l}\text { Degrees of } \\
\text { Freedom }\end{array}$ & $\begin{array}{l}\text { Sum of } \\
\text { Squares }\end{array}$ & $\begin{array}{l}\text { Mean } \\
\text { Square }\end{array}$ & $\begin{array}{l}\text { Ratio of } \\
\text { Variances, F }\end{array}$ & $F, 95 \%$ \\
\hline Particle size & 3 & 1.43 & 0.48 & 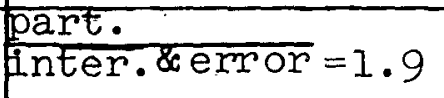 & $9 \cdot 3$ \\
\hline Concentration & 1 & $I .50$ & 1.50 & conc. & 10.1 \\
\hline Interaction & 2 & 0.08 & 0.04 & & \\
\hline Error & 1 & 0.21 & 0.21 & & \\
\hline Total & 7 & 3.29 & & & \\
\hline
\end{tabular}

TABLE 10

ANALYSIS OF VARIANCE - EFFECT OF ENERGY RATE

\begin{tabular}{l|c|c|c|c|c}
\hline $\begin{array}{l}\text { Source of } \\
\text { Variation }\end{array}$ & $\begin{array}{l}\text { Degrees of } \\
\text { Freedom }\end{array}$ & $\begin{array}{l}\text { Sum of } \\
\text { Squares }\end{array}$ & $\begin{array}{l}\text { Mean } \\
\text { Square }\end{array}$ & $\begin{array}{l}\text { Ratio of } \\
\text { Variances, F }\end{array}$ & F, 95\% \\
\hline Energy rate & 1 & 2.76 & 2.76 & energy $=30.7$ & 6.0 \\
$\begin{array}{l}\text { Remainder } \\
\text { Total }\end{array}$ & $\frac{6}{7}$ & $\frac{0.54}{3.30}$ & 0.09 & & \\
\hline
\end{tabular}


The two runs with large particle size were at the same concentration; therefore this variance is given as error. When the variances in particle size and concentration are compared to the sum of interaction and error, neither appears to be significant.

A second analysis of variance, given in Table 10, was made to determine if the effective fission energy rate is significant. When the variance of particle size and interaction are combined as an estimate of the experimental error, the $F$ test ratio shows a definite energy input effect on $G\left(\mathrm{H}_{2}\right)_{f}$. This could be due to experimental error. Since the yield of hydrogen is obtained from the difference between the hydrogen rate with fissions and that without fissions, experimental error in $\mathrm{G}\left(\mathrm{H}_{2}\right)$ increases as we move towards lower energy rates (see Figure 19). For this reason, an energy-weighted average of the $G\left(H_{2}\right)$ f values should be a better estimate of the true value than the normal average. By multiplying the G-values by their fission energy rate, and then dividing the sum of these weighted values by the sum of the energy, a value of $G\left(H_{2}\right)$ f of 2.15 was obtained. The best estimate of the experimental error is that error variance used in Table 10, because it includes all random errors but this energy dependence. With 90 per cent confidence, the value of $G\left(H_{2}\right)$ f $1 \mathrm{~s} 2.15 \pm .21$, which is in good agreement with that obtained by drawing the best line through the data. 
Effect of stripping

The extent to which the recombination of molecular hydrogen occurs to form water by the reaction:

$$
\mathrm{H}_{2}+\mathrm{OH}=\mathrm{H}_{2} \mathrm{O}+\mathrm{H}
$$

depends upon both the concentration of the hydrogen and that of the hydroxyl radical. The concentration of the free radicals depends almost entirely on the intensity of the gamma and neutron radiation for few radicals escape the track of a fission fragment. The concentration of hydrogen, however, is controlled by diffusion from the liquid to the gas, which depends on both the rate of bubbling and the stirring efficiency. Estimate of the gamma intensity in the reactor from the hydrogen evolution rate gave a dose rate of more than $10^{22} \mathrm{ev} / 1 / \mathrm{min}$. Since this was about 10 times that which was obtained with the co ${ }^{60}$ source, the effect of stripping should be more pronounced in the reactor experiments.

The runs made with $\mathrm{UO}_{2} \mathrm{SO}_{4}$ solutions, shown in Figure 20, illustrate the effect of stripping. The low yield for man 8 shows that stirring was a necessary part of the stripping process. The stirring and bubbling rates for run 5 would probably have been sufficient if the gamma intensity had been that of the $\mathrm{Co}^{60}$ source, but in the reactor more than half of the hydrogen was recombined. In an attempt to prevent the splashing that occurred in run 7 , stirring was so slow in run 21 that most of the hydrogen was recombined. 


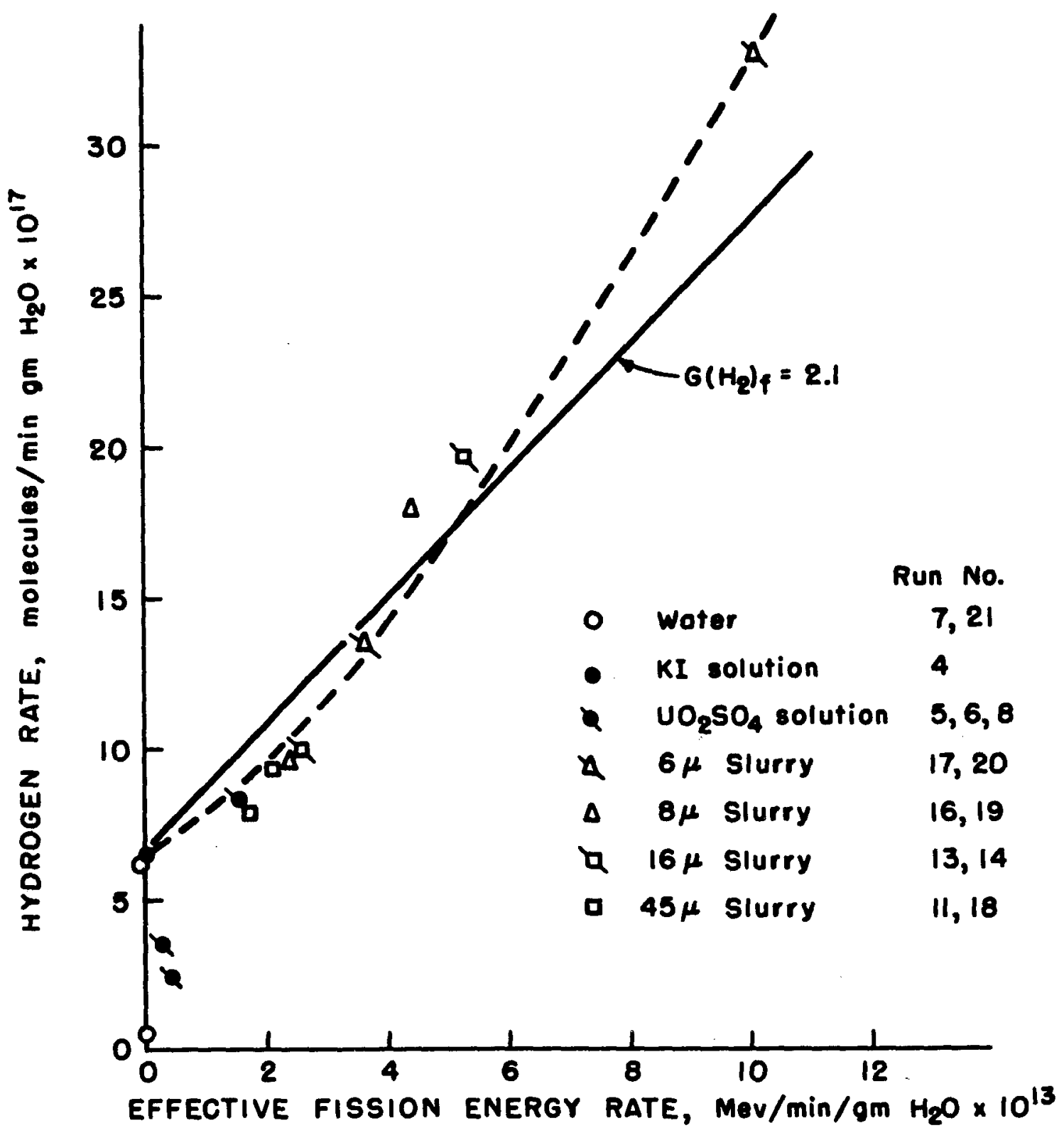

Figure 20. Hydrogen yield. 
There should have been no effect of stripping for mun 4 because the presence of lodide $10 \mathrm{n}$ in the water prevents the recombination reaction. The fact that the datum point for run 6 is consistent with that of run 4 and the slurry data indicates that the stripping was as good as that for the slurry runs.

It was observed that the presence of solid particles induced a more uniform stripping action than that which could be obtained in pure liquid. Moreover, the bubbling and stirring rates for the slurry runs were even higher than for mun 6. The fact that a line can be drawn through all the data supports the hypothesis that recombination was not important. However, if there were a little recombination, the effect would be more pronounced for the runs in which the hydrogen yields were low than in those which were high. This could be the reason that the data points for low fission energy rates are below the line in the flgure.

\section{Energy transfer}

Energy could be absorbed in the solid and transferred to the liquid in the form of gamma rays or charged particles. On the other hand, the solld may act as a shield, absorbing some of the energy that would have been absorbed in the liquid. No allowance was made for elther of these effects on the data.

Experiments with the $\mathrm{Co}^{60}$ source to provide $\gamma$-radiation only have shown that there is no significant effect of the 
presence of the solid particles in the water on expected decomposition rates. Measurements of the hydrogen yield, shown in Figure 21, with and without solid gave a value for $G\left(\mathrm{H}_{2}\right)$ between 0.4 and 0.5 in good agreement with the expected value of 0.45 (4). Recombination was significant at low flow rates; the hydrogen was so dilute at high flow rates that analysis was difficult. The oxidation rate of ferrous-cupric dosimetry solution was also measured; the results are given in Figure 22. No appreciable effect of solid was observed, although a change of less than 20 per cent probably would not have been detected. These results do not necessarily prove that there is no effect of solid because most of the gammas in the reactor have energies greater than the $1 \mathrm{Mev}$ energy level of $\mathrm{Co}^{60}$.

If there were an effect of energy transfer, it would show up as a concentration effect. Increasing the concentration should have increased the energy transfer, but the analysis of varlance in Table 9 shows no effect of concentration.

No allowance was made for energy other than fission fragment energy that was released in the fission process within the sample tested. If all this energy were absorbed in the slurry, It would amount to an increase in energy of about 12 per cent. In the case where half of the fission fragment energy is absorbed in the solid, the effect of this other energy would be an increase of the hydrogen rate 


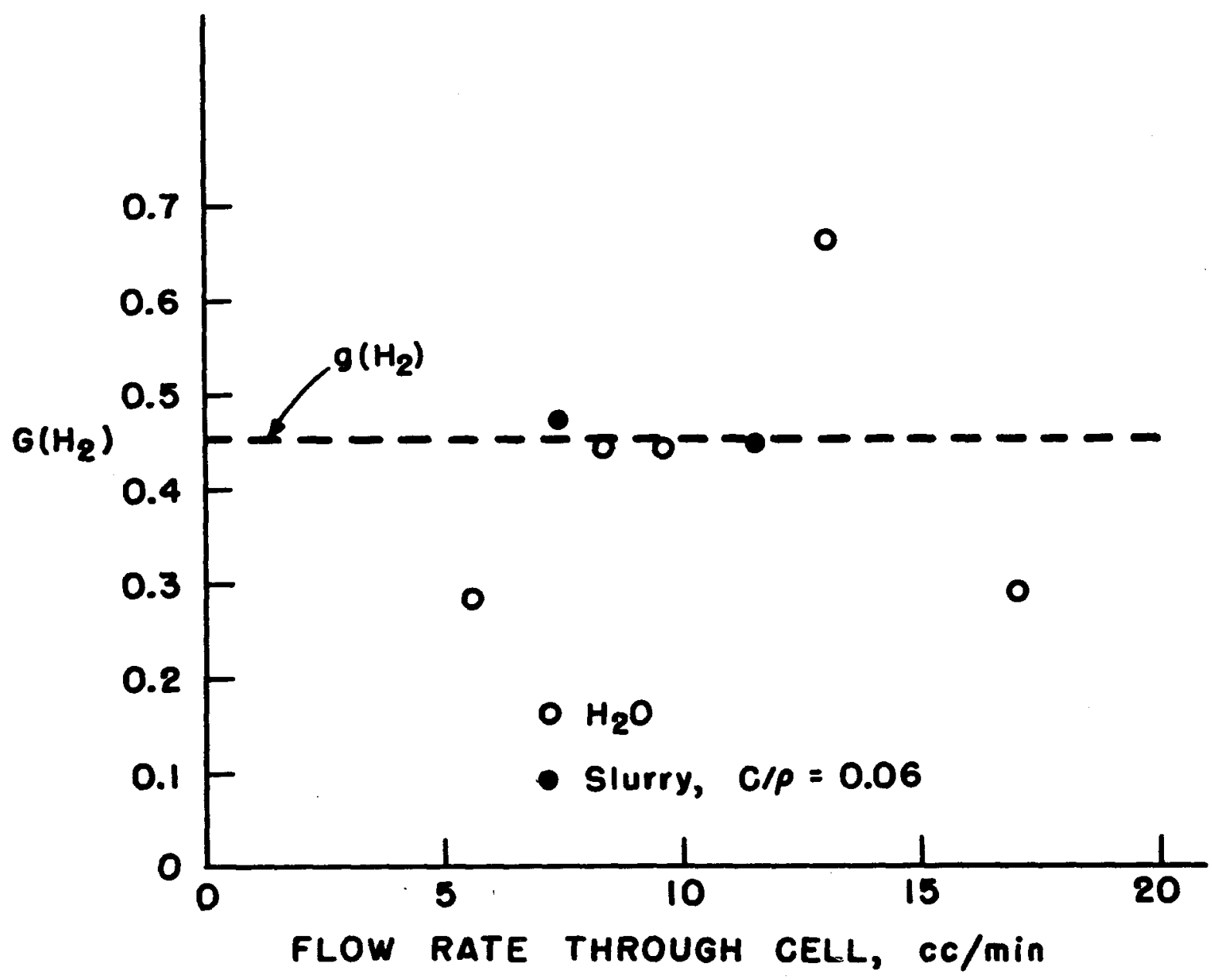

Figure 21. Hydrogen yield under gamma irradiation.

๘ 


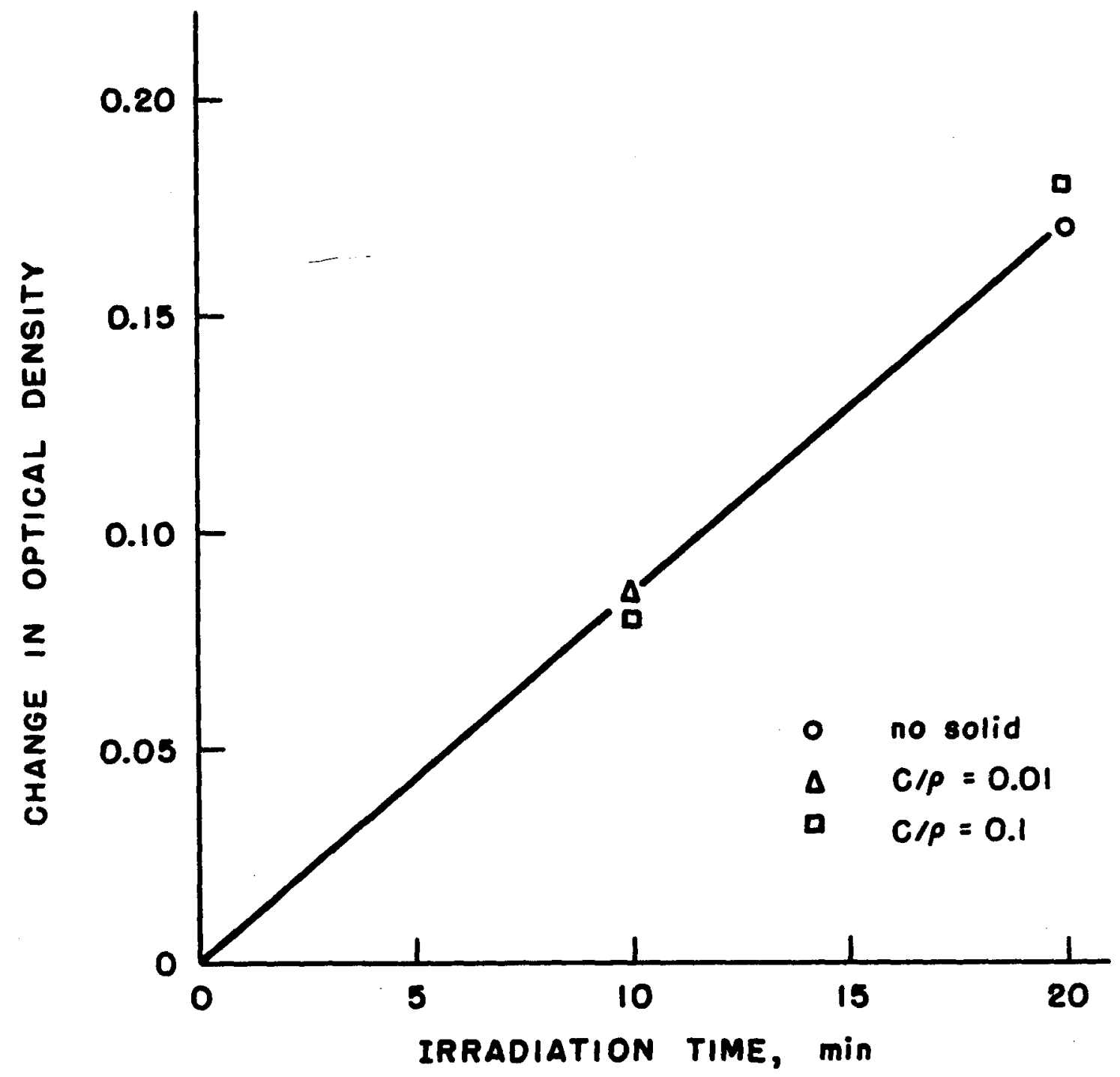

Figure 22. Effect of solids on the oxidation of $\mathrm{Fe}-\mathrm{Cu}$ dosimetry solution. 
by about 5 per cent. Since most of this energy escapes the system or else is delayed over a period of time, its effect should be negligible. Dose rate with mixed radiations

One would suspect that the hydrogen yield due to gammas and that due to the fissions are not entirely independent of each other. If so, the interaction effect should increase as the dose rate increases. At high dose rates more free radical reactions could occur than at low intensities--the result would be higher hydrogen yields. Not enough work has been done at high dose rates with mixed radiations to be able to attribute the observed increase in hydrogen yleld with dose rate to this effect. Alternate models

Calculation of the fraction of fission energy deposited in the water was based on a particular range-energy relationship and particular values of the ranges. Sears and Steinberg (32) have calculated the fraction of energy that should escape a slurry of $\mathrm{UO}_{2}$ in water for two types of range-energy relationships--the rate of energy deposition is constant (linear) and the rate is proportional to velocity (quadratic). In addition, they have allowed for the case in which the G-value might increase towards the end of the fission fragment track. The data of this experiment were recalculated with these alternate models and plotted on the graphs in Figure 23. In order to 


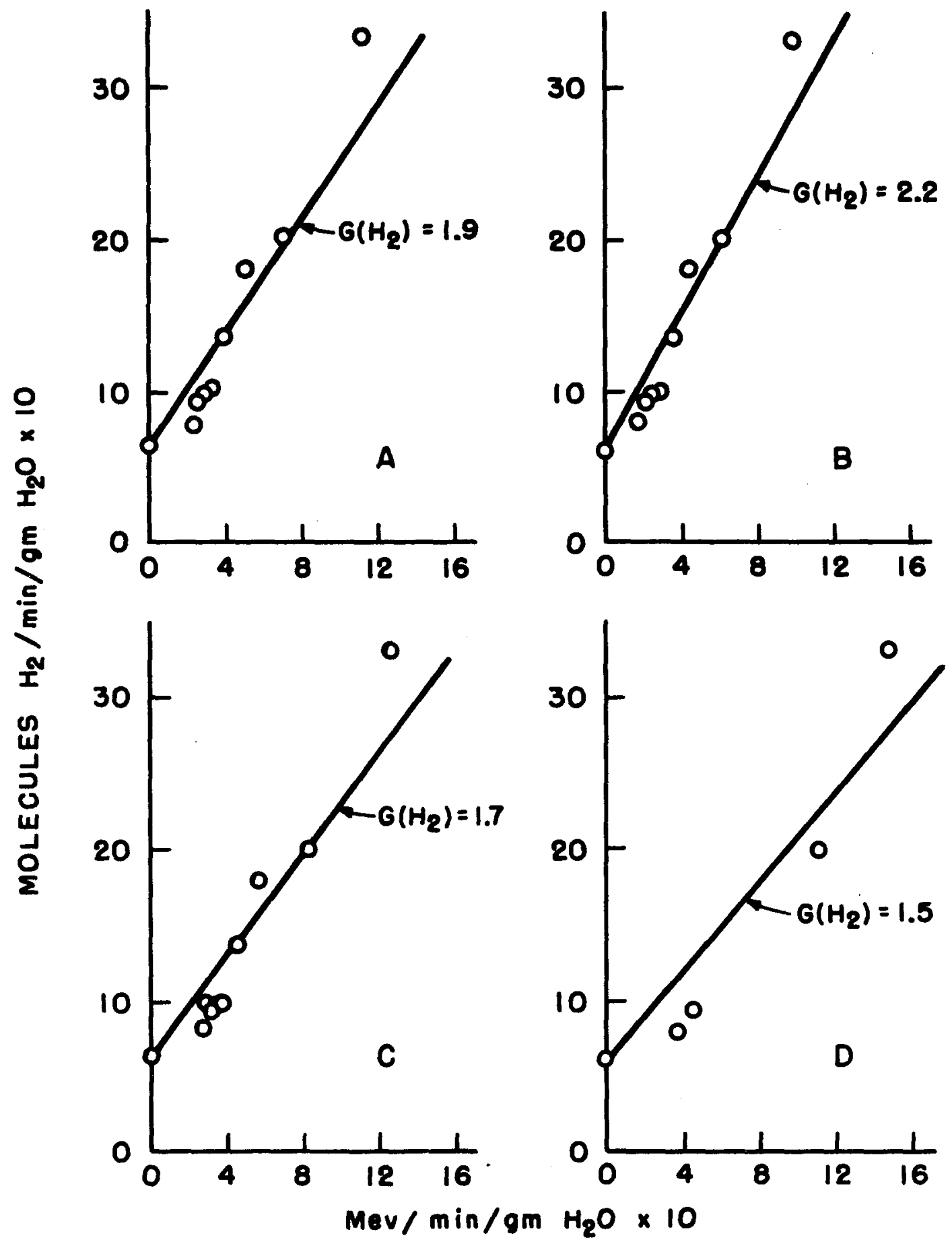

Figure 23. Alternate models of range-energy relationships.

(Range in solid $=10 \mu$, range in water $=20 \mu$ )
A. Three-holves $E$, constont $G$
B. Quadratic $E$, constant $G$
C. Linear E, constant $G$
D. Quadratic E, Linear $G$ 
compare the effects of these models on the data to that of the model used in this thesis, (three-halves), range values of 10 and 20 microns (62) were used for the solid and llquid, respectively, for all the models shown in the figure. Ranges of 8 and 24 microns were used elsewhere.

All the models for which $G$ is constant give about the same fit to the data, but the G-values are different. As one would predict, the three-halves model gives a value about half-way between that of the other two approximations. For the case in which $G$ is not a constant, the data do not satisfactorily fit the straight line. Besides, the value of $G$ is lower than that which would be expected.

Note that by changing the range values by about 20 per cent in the direction that allows more energy to be deposited in the water, the G-value is decreased by about 10 per cent. The G-value is therefore not very sensitive to the range values used. The same type of effect would have been observed if the particle diameters were changed instead of the ranges. Any error in measuring the particle size should not appreciably change the value of $G\left(\mathrm{H}_{2}\right)$ found in this experiment.

Fraction of particles escaping the solid

The relative number of the fission fragments which should be trapped in the solid phase of the slurry has been calculated with the Monte Carlo technique. The results for the case in which the ratio of the range in liquid to that 
In solid is 3.0 are given in Figure 3. Those for other ratios are given in Figure 4. At zero concentration only those fragments which do not escape the parent sphere are trapped in the solid. As the concentration increases, more and more fragments are trapped in neighboring spheres. The fraction that is trapped also depends upon the ratio of the range in liquid to that in solid, for as this ratio increases, the probability of the fragment being stopped in the liquid decreases.

Two types of measurements of the fraction of fragments escaping in the solid were made in these experiments. The first was that of the volatile fission products in the gas stream, which was monitored for its activity. Since the count rate recorded was only a relative measure of the number of fission products, the value for mun 16 was placed on the line in Figure 24; the rest of the results shown in the flgure are relative to $1 t$. These results are in very good agreement with the computed values for a range ratio of 3.0 .

The other method of measuring this fraction is by the relative amounts of the molybdenum-99 found in the solid and in the liquid. Since some of the fragments could be readsorbed "on the surface of the solid, one might expect that the results would indicate that a higher fraction in the solid than predicted. These results, shown in Table 14 do give a somewhat higher value for the Mo99, and the 


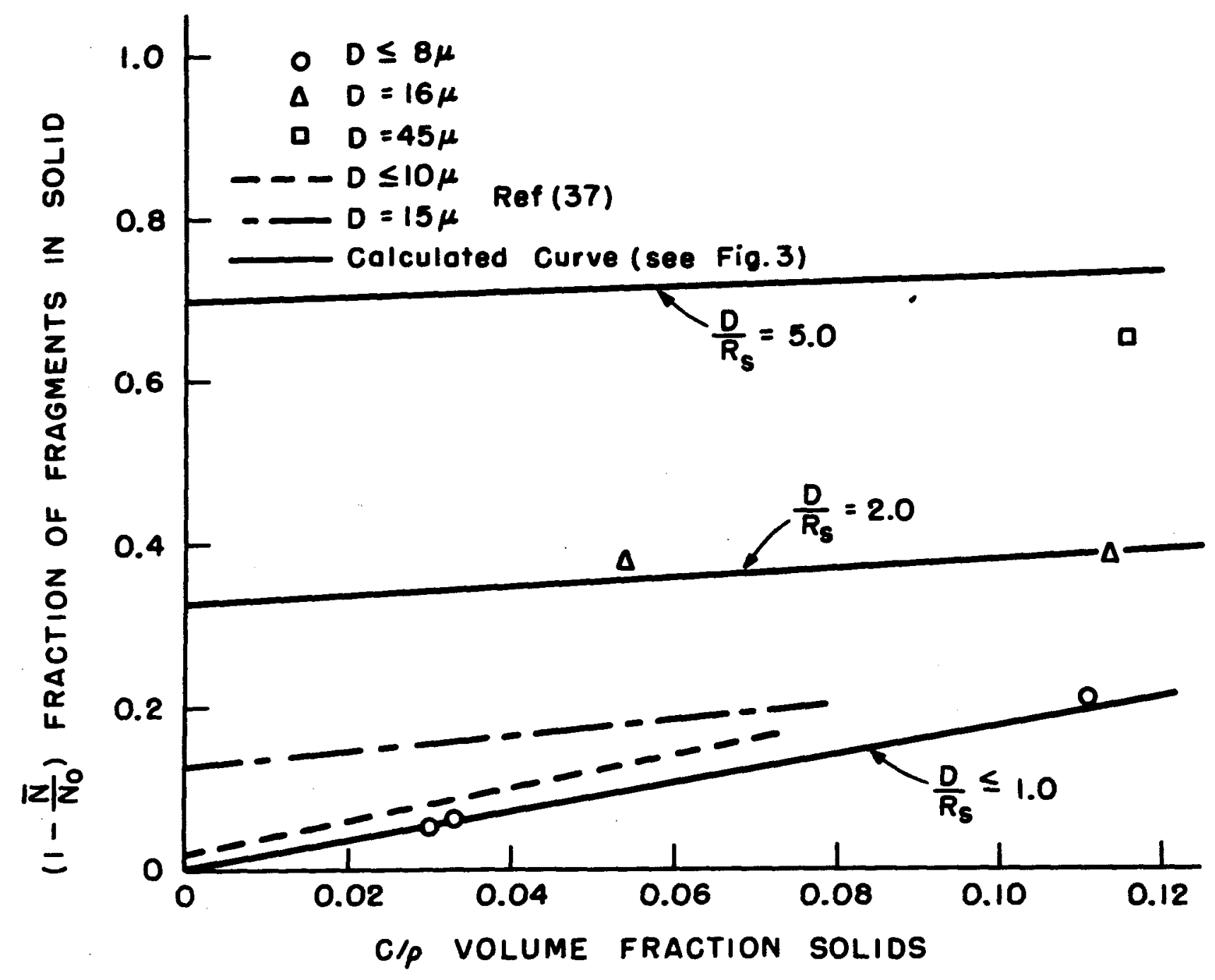

Figure 24. Fission products in the solid. 
results of the $\mathrm{Ce}^{144}$ show how strong this readsorbtion could be. The results of Hermans and Plas (37), who used active charcoal to adsorb the fission products, are shown in Figure 21. The line for the 15 micron particles is somewhat lower than it should be. Comparison with other data

Very little data have been obtained on the effects of fission recoil fragments in water. Only one measurement for a slurry system has been reported (27); when corrected for particle size and concentration, this value for $G\left(\mathrm{H}_{2}\right)_{f}$ becomes about 1.0. Recombination of the hydrogen with free radicals may be the reason that this value is so much less than that found in this work. The data that have been obtained from solutions of uranium (5) Indicate a value of $G\left(\mathrm{H}_{2}\right)_{f}$ equal to 1.8 for infinitely dilute solutions. However, increasing the concentration of uranium in the solution caused a decrease in the decomposition. There was an additional effect of $\mathrm{pH}$; at a constant uranium concentration, hydrogen yields increased with an increase in $\mathrm{pH}$. Such results indicate that the uranium acted as a catalyst for the recombination to form water and that this recombination was sensitive to $\mathrm{pH}$. To show the effect of $\mathrm{pH}$, the effects of concentration and $\mathrm{pH}$ had to be separated. These data for uranium solutions highly enriched in $\mathrm{U}^{235}$ were corrected for the concentration 
effect by adding the quantity, $0.05(\mathrm{C})^{1 / 2}$, where "C" is the concentration in grams uranium per liter, to the reported value of $\mathrm{G}\left(\mathrm{H}_{2}\right)$. These corrected values were then plotted on the graph in Figure 25. Extrapolation of the line through these data to a $\mathrm{pH}$ of 7.0 ylelds a value of hydrogen evolution rate that is in good agreement with that obtained in this work.

Although no other data are available that show the effects of fission fragments, the result of this work may be compared to the results of other types of radiation. Pucheault (63) has recently correlated hydrogen yields from water due to heavy particle radiations as a function of their initial rate of energy deposition. These data, along with that obtained in this work, are plotted on the graph in Figure 26. The maximum value of the hydrogen yield should not exceed half of 4.7 , the total number of molecules decomposed per $100 \mathrm{ev}$. absorbed by the water (3). Even this would occur only if all the hydrogen atoms recombined with each other. The value of $G\left(\mathrm{H}_{2}\right)$ due to fission recoils, for which the initial rate of energy loss is about $5,000 \mathrm{kev} / \mu$, agrees quite well with the extrapolated curve. 


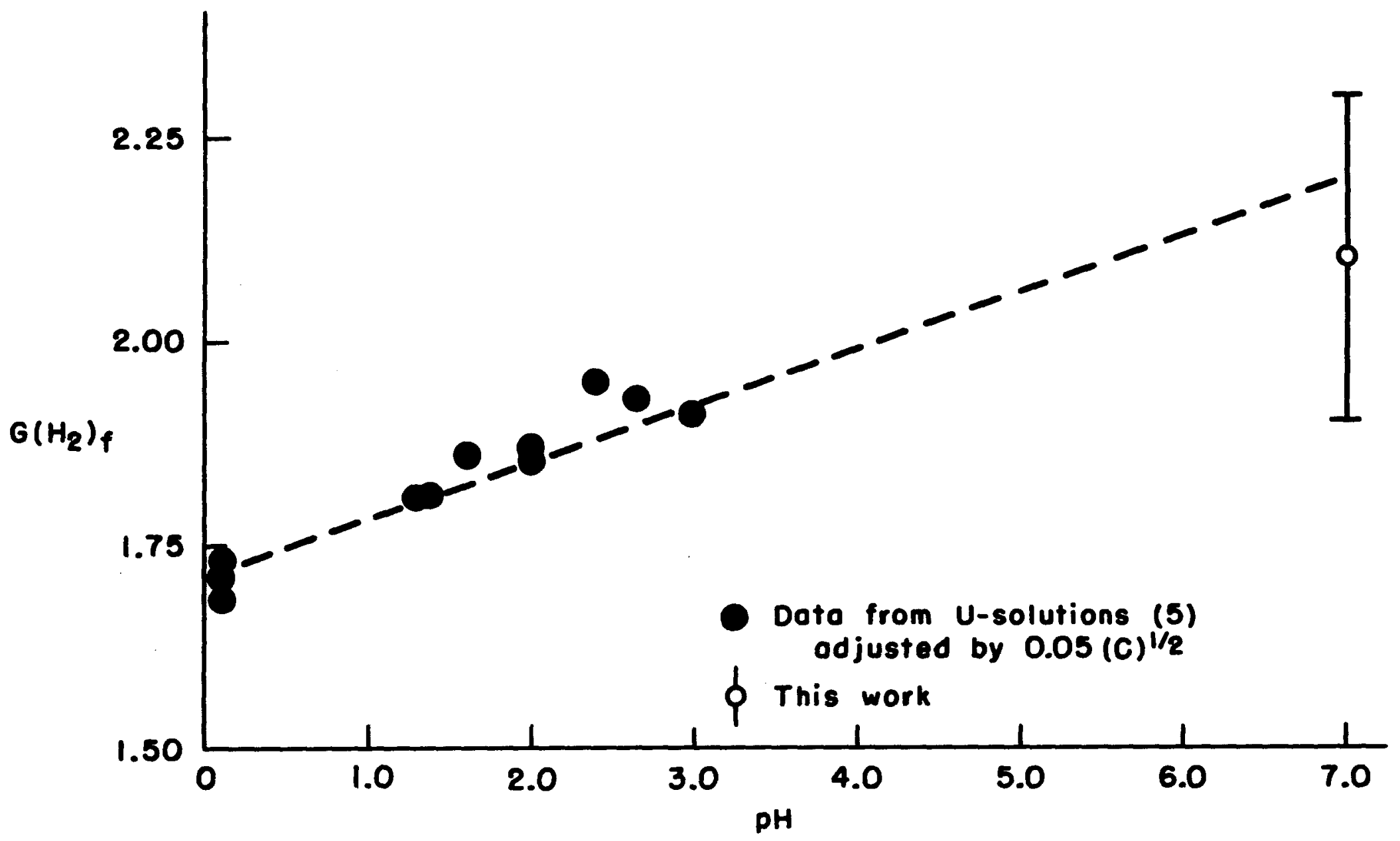

Figure 25. Effect of $\mathrm{pH}$ on hydrogen yields due to fission recoils.

i 


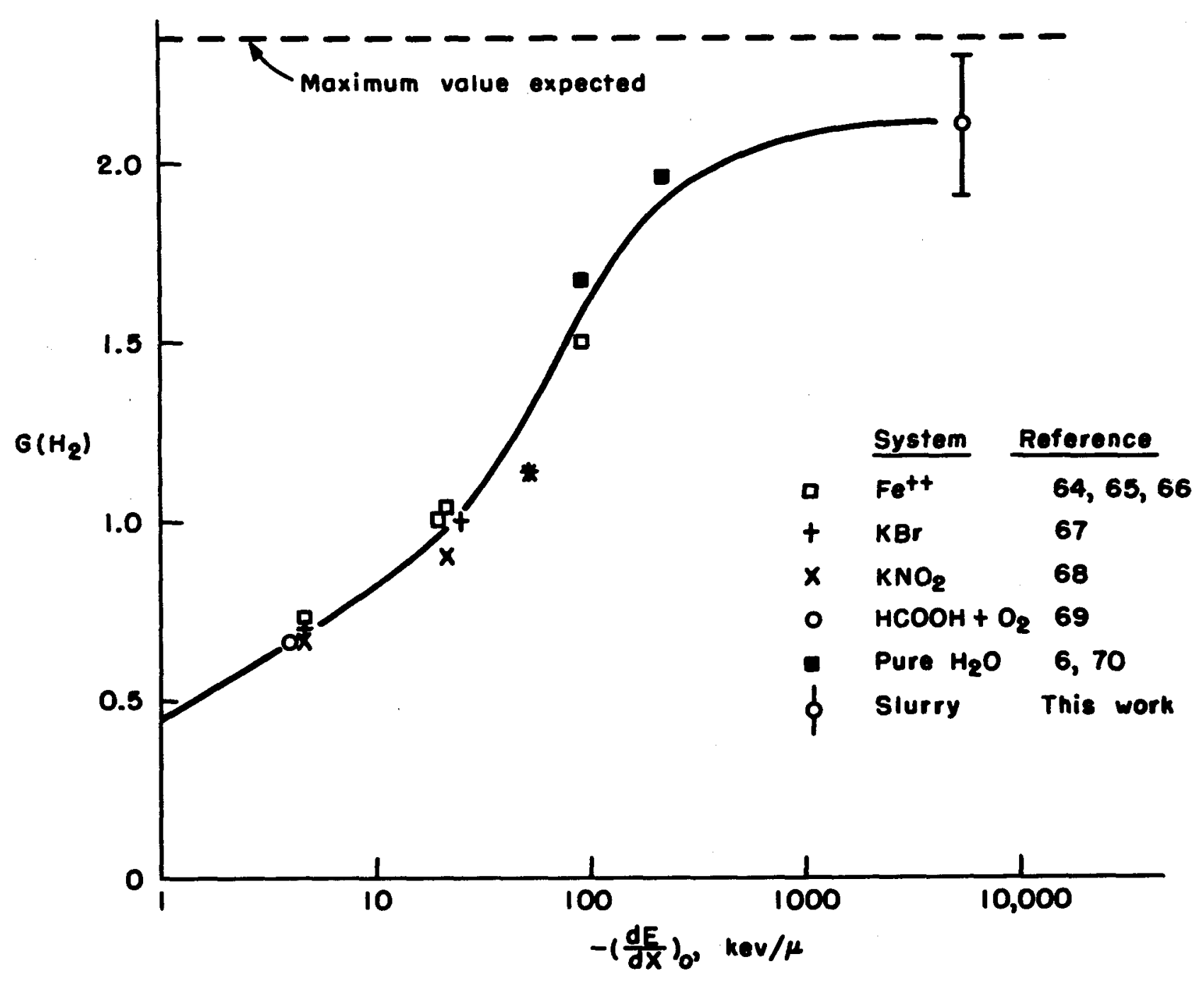

Figure 26. Hydrogen yields for various LET. 


\section{CONCLUSIONS}

The result of these experiments, a value for $G\left(\mathrm{H}_{2}\right)_{f}$ of $2.1 \pm 10 \%$, agrees well with the value which data at lower LET predict. This value is higher than that previously reported for solutions of uranium, but catalytic recombination could have been the reason for the lower value. This measured value is therefore probably $g\left(\mathrm{H}_{2}\right)_{f}$, the number of hydrogen molecules escaping from the track of the fission recoil particle for every $100 \mathrm{ev}$. deposited in the water. It also represents the value of $\mathrm{G}\left(-\mathrm{H}_{2} \mathrm{O}\right)_{f}$, the number of molecules of water decomposed by fission recoil energy in the water under steady-state conditions in a slurry reactor.

The model used in these experiments to correlate the data has been based on the best information available. Because a computer was used to carry out the calculations, a better approximation to the range-energy relationship than those previously reported could be used. Since the computer results do agree with what was expected from information in the literature, there were no gross errors in the computer program. This hypothesis is supported by the fact that the experimental data on the fraction of volatile fission products escaping the solid agree with calculated values.

The data are not really a test of the model (E proportional to the three-halves power of $R$ minus $x$, and $G$-values 
constant over the path length) because a standard reference value for the hydrogen yield, $g\left(\mathrm{H}_{2}\right) f$, is not known. However, the model has satisfactorily accounted for the effects of particle size and concentration. Since the analysis of variance has shown that there is no effect of particle size, other than that predicted by the model, the hydrogen yield was probably constant over the path length of the fission recoil.

In addition to the effects of particle size and concentration, which were predicted by the model, there was an observed effect of energy deposition rate, which was probably a result of experimental conditions. At low rates, recombination could have been the cause, while at high rates, the effect of dose rate could have been important. Under the conditions of this experiment, in which even at the highest rate of effective fission energy deposition, the energy absorbed by the water due to gamma and neutron background was greater than that due to fissions, little can be concluded as to the exact cause of this energy effect.

The fact that a reasonable value for $G\left(\mathrm{H}_{2}\right)_{f}$ was obtained under such unfavorable conditions suggests that the method for obtaining the hydrogen yleld was quite satisfactory. The errors in the varlous measurements were not excessive; additional experiments under similar conditions would not appreciably reduce the error in the result. These experiments have demonstrated that a slurry of fissionable 
material can be used as a source of fission product recoll energy •

The results of the Monte Carlo calculation are a significant contribution for future research in this field. Because an equation has been found to correlate the calculated data, the fraction of energy deposited in the fluid can be calculated for a number of slurry systems. Until the behavior of fluids under heavy particle radiation is better understood, this calculation is the only way of knowing the fraction of energy deposited in the fluid. For studies of systems in which g-values are not constant over the path length, the calculated fission recoil escape energy spectrum may be used to modify the model. 


\section{RECOMMENDATIONS}

The effect of very densely lonizing particles on water is still not very well understood. In this work only one product, hydrogen, was measured, whereas knowledge of all the products of the irradiation is necessary in order to compare the effects of this type radiation with that of the less densely lonizing type. Heretofore, one reason that the effects of fission recoils in solutions have not been more thoroughly investigated has been the lack of an inert source. Another has been the lack of reliable information on the fraction of fission energy deposited in the fluid. Now that it has been demonstrated that a slurry can be used as a source and the energy deposition relationship calculated, more use should be made of the slurry source.

Although this equipment was designed to measure a particular product using a particular reactor, the principles can be applied to other systems. One of the flrst changes in the experiment should be that of increasing the ratio of fission energy to background energy. For a reactor irradiation, this could be accomplished by increasing the concentration of fissionable material in the slurry. If the detection methods are sensitive enough, perhaps a neutron source with less background than a reactor could be used, e.g., a Po-Be type source or a particle accelerator with a target for the source. 
If the background were low enough, the fraction of fission recoll energy escaping to the fluid could be measured experimentally using a system for which G-values are somewhat independent of LET. Dewhurst and Schuler (71) have shown that the decomposition of either hexane or cyclohexane by radiation with LET values from 0.02 to $5 \mathrm{ev} . / \AA$ is independent of the linear energy transfer of the radiation. If the G-values for these systems were constant up to the LET of fission recoil fragments, then by knowing the total energy input, one could easily determine the effective energy in a slurry.

None of the gas lines in this equipment were shielded to prevent the escape of fission product activity. For future work in which the stripping technique is used, the outlet line should be shielded, especially if the rate of fissions in the sample is increased.

The purge Iine may not have been necessary; in this work it was only partially successful in keeping out oxygen. The advantages of a second seal may not be enough to justify the additional mass required, since this mass increases the activation by neutrons.

Elimination of the gear box would also reduce the amount of mass in the high flux region. In a vertical beam hole or in a swimming pool reactor, the motor drive mechanIsm could be located above the slurry, so that direct drive would be possible. 


\section{BIBLIOGRAPHY}

(1) Hart, E. J., Platzman, R. L., "Mechanisms in Radiob1ology," Vol. I, Chap.2, Academic Press, Inc., New York, 1961.

(2) Allen, A. O., "The Radiation Chemistry of Water and Aqueous Solutions," D. Van Nostrand Co., Inc., Princeton, N. J., 1961.

(3) Hart, E. J., Platzman, R. L., "Mechanisms in Rad1obiology," Vol. I, p. 188, Academic Press, Inc., New York, 1961.

(4) Allen, A. O., "The Radiation Chemistry of Water and Aqueous Solution," p. 44, D. Van Nostrand Co., Inc., Princeton, N. J., 1961.

(5) Boyle, J. W., et al., Int. Conf. Peaceful Uses Atomic Energy, Geneva 7, 576 (1956).

(6) Hart, E. J., McDonell, W. R., Gordon, S., Int. Conf. Peaceful Uses Atomic Energy, Geneva 7, 593 (1956).

(7) Gordon, S., Hart, E. J., 2nd. Int. Conf. Peaceful Uses Atomic Energy, Geneva 29, 13 (1958).

(8) Hochanade1, C. J., J. Phys. Chem. 56, 587 (1952).

(9) Schwarz, H. A., Losee, J. P., Allen, A. O., J. Am. Chem. Soc. 76, 4693 (i954).

(10) Hochanadel, C. J., Int. Conf. Peaceful Uses Atomic Energy, Geneva 7, 521 (1956).

(II) Alexander, J. M., Gazdik, M. F., Phys. Rev. 120, 874$876(1960)$.

(12) "Shipping Port Pressurized Water Reactor," p. 183, Addison-Wesley Pub. Co., Inc., Reading, Mass., 1958.

(13) Robertson, R. F. S., Int. Conf. Peaceful Uses Atomic Energy, Geneva 7, 556 (1956).

(14) Harrer, J. M., et al., 2nd. Int. Conf. Peaceful Uses Atomic Energy, Geneva 9, 264 (1958).

(15) U. S. Atomic Energy Commission, ANL-5800, 22 (1958).

(16) Lane, J. A., MacPherson, H. G., Maslan, F., "Fluid Fuel Reactors," p. 107, Addison-Wesley Pub. Co., Reading, Mass., 1958. 
(17) McDuff1e, H. F., et al., J. Phys. Chem. 62, 1030 (1958).

(18) Lane, J. A., MacPherson, H. G., Maslan, F., "Flu1d Fuel Reactors," p. 436, Addison-Wesley Pub. Co., Reading, Mass., 1958.

(19) Ibid., pp. 1, 9, 135.

(20) Benveniste, J., et al., 2nd. Int. Conf. Peaceful Uses Atomic Energy, Geneva 9, 415 (1958).

(21) Kreeyger, P. S., et al., 2nd. Int. Conf. Peaceful Uses Atomic Energy, Geneva 9, 427 (1958).

(22) Zajic, V., Pfann, J., Gregor, J., 2nd. Int. Conf. Peaceful Uses Atomic Energy, Geneva 9, 441 (1958).

(23) Petrick, M., Marchaterre, J. F., U. S. Atomic Energy Commission, ANL-6148, 1960.

(24) Freund, G. A., et al., U. S. Atomic Energy Commission, ANL-6248, 1960.

(25) U. S. Atomic Energy Commission, ORNL-1814, 144 (1954).

(26) U. S. Atomic Energy Commission, ORNL (CF-59-7-129), p. 61 .

(27) Lane, J. A., MacPherson, H. G., Maslan, F., "Fluid Fuel Reactors," pp. 183-189, Addison-Wesley Pub. Co., Reading, Mass., 1958.

(28) McDole, C. J., Westinghouse Electric Corporation, WCAP-277, 1956.

(29) Gardner, D. G., Nuc. Sc1. and Engr. 6, 487-492 (1959).

(30) Long, G., United Kingdom Atomic Energy Authority, AERE C/M-345, 1958.

(31) Weber, H., Hirsch, C., 2nd. Int. Conf. Peaceful Uses Atomic Energy, Geneva 9, 196 (1958).

(32) Sears, J., Steinberg, M., U. S. Atomic Energy Commission, BNL-695(T-238), 1961 .

(33) Edwards, C. G., Moseley, F., United Kingdom Atomic Energy Authority, AERE C/R 2710, 1958. 


\section{BIBLIOGRAPHY (contd.)}

(34) Steinberg, M., Powell, J. R., Green, L., U. S. Atomic Energy Commission, BNL $602(T-175), 1961$.

(35) Compere, E. L., et al., U. S. Atomic Energy Commission, TID-7622, 1961.

(36) Sowden, R. G., et al., U. S. Atomic Energy Commission, TID-7622, 1961.

(37) Hermans, M. E. A., Plas, von der, Th., Nuc. Sci. and Engr. 2, 224-226 (1957).

(38) Bohr, N1els, D. Kgl. Danske Vidensk. Selskab, Math.fys. Medd. XVIII, 8, 1948.

(39) Livingston, M. S., Bethe, H. A., Rev. Modern Physics 9, 245 (1937).

(40) Winsberg, L., Alexander, J. M., Phys. Rev. 121, 518528 (1961).

(41) Harvey, B. G., Ann. Rev. Nuc. Sc1. 10, 235-258 (1960).

(42) Fulmer, C. B., Phys. Rev. 108, 113 (1957).

(43) Niday, J. B., Phys. Rev. 121, 1471-1483 (1961).

(44) Tobias, M.; U. S. Atomic Energy Commission, ORNL CF-609-44 $(1960)$.

(45) Crosby, J., et al., "Fast Neutron, Thermal Neutron, and Gamma Ray Flux--CP-5 Thermal Column," 1959, Unpublished paper, ANL.

(46) Dodd, R. E., Robinson, P. L., "Experimental Inorganic Chemistry, " p. 133, Elsevier Pub. Co., Amsterdam, 1954.

(47) Blomgren, R. A., Proceedings Sixth Hot Laboratories and Equipment Conference, 229 (1958).

(48) Crouthamel, C. E., et al., U. S. Atomic Energy Commission, ANL-6340 (1961).

(49) "Recent Developments in Mineral Dressing," Chap. 2, p. 52, Heywood, H., Institution of Mining and Metallurgy, London, 1953.

(50) Ibid., p. 49. 
BIBLIOGRAPHY (contd.)

(51) Abraham, B. M., Flotow, H. E., Carlson, R. D., Anal. Chem. 29, 1058 (1957).

(52) Leddicotte, G. W., U. S. Atomic Energy Commission, ORNL CF 60-11-124, 1961 .

(53) Herdan, G., "Small Particle Statistics, : 2nd. ed., Academic Press, New York, 1960.

(54) Hodgman, Charles D., (ed. In chief), "Handbook of Chemistry and Physics," 34th Ed. 2006, Chemical Rubber Publishing Co., Cleveland, Ohio, 1953.

(55) Sheehy, M. L., Indust. and Eng. Chem. 24, 1060 (1932).

(56) Bayer, E., "Gas Chromatography," Elsevier Publishing Co., Amsterday, 1961.

(57) Van Slyke, D. O., Ne11l, J. M., J. Biol. Chem. 61, $523(1924)$.

(58) Hart, E. J., J. Am. Chem. Soc. 73, 68 (1951).

(59) Baker, S., Argonne National Laboratory, Argonne, Ill., private communication, 1962.

(60) Stehn, John F., Nucleonics 18, 187 (1960).

(61) Hart, E. J., Walsh, P. D., Int. Conf. Peaceful Uses Atomic Energy, Geneva 29, 38 (1958).

(62) Segré, E., Wlegand, C., Phys. Rev. 70, 808 (1946).

(63) Halssinsky, M., (ed.), "The Chemical and Biological Action of Radiations," 5th series, Chap. 2, p. 56 Pucheault, J., Academic Press, Inc., London, 1961).

(64) Schuler, R. H., Allen, A. O., J.Am. Chem. Soc. 79, 1565 (1957).

(65) Miller, N., Wilkinson, J., Trans. Far. Soc. 50, 690 (1954).

(66) Barr, N. F., Schuler, R. H., J. Phys. Chem. 63, 808 (1959).

(67) Garrison, W. M., U. S. Atomic Energy Commission, UCRL 3653 . 


\section{BIBLIOGRAPHY (contd.)}

(68) Schwarz, H. A., Caffrey, Jr., J. M., Schules, G., J. Am. Chem. Soc. 81, 1801 (1959).

(69) Garrison, W. M., Weeks, B. M. , Ward, J. O., Bennet, W., J. Chem. Phys. 27, 1214 (1957).

(70) Anta, M. C., Lefort, M., J. Ch1m. Phys. 51, 29 (1954).

(71) Dewhurst, H. A., Schuler, R. H., J. Am. Chem. Soc. 81, 3210 (1959). 
NOMENCLATURE

\begin{tabular}{|c|c|}
\hline A & Atomic welght \\
\hline$A_{R}$ & Atomic welght of the recoling atom \\
\hline $\mathrm{B}$ & $\begin{array}{l}\text { Range-energy relationship for low velocity frag- } \\
\text { ments in a medium of lower atomic weight }\end{array}$ \\
\hline $\mathrm{C}$ & $\begin{array}{l}\text { Concentration of solids in the slurry in grams per } \\
\text { cc. of slurry }\end{array}$ \\
\hline$D$ & $\begin{array}{l}\text { Diameter of a solid particle in the slurry in } \\
\text { microns }\end{array}$ \\
\hline$e$ & Charge of an electron \\
\hline $\mathrm{E}$ & Energy of a fission fragment \\
\hline$E(x)$ & $\begin{array}{l}\text { Energy of a fission fragment that has travelled a } \\
\text { distance } x\end{array}$ \\
\hline$E_{0}$ & Initial energy of a fission fragment \\
\hline $\bar{E} / E_{0}$ & $\begin{array}{l}\text { Average fraction of fission fragment energy escap- } \\
\text { ing the solid in a slurry }\end{array}$ \\
\hline$F$ & Ratio of variances \\
\hline $\mathrm{F}_{\mathrm{n}}$ & $\begin{array}{l}\text { Fraction of the initial count rate in the sedimen- } \\
\text { tation column at the } \mathrm{n}^{\text {th }} \text { measurement }\end{array}$ \\
\hline g & Gravitational constant \\
\hline$g(x)$ & $\begin{array}{l}\text { The number of molecules of } X \text { formed in the track } \\
\text { that subsequently escape the track of an ionizing } \\
\text { particle for every } 100 \mathrm{ev} \text {. absorbed }\end{array}$ \\
\hline$G(x)$ & $\begin{array}{l}\text { The number of molecules of } X \text { measured for every } 100 \\
\text { ev. absorbed }\end{array}$ \\
\hline$G(x)_{f}$ & The G-value due to fission recoll energy \\
\hline
\end{tabular}




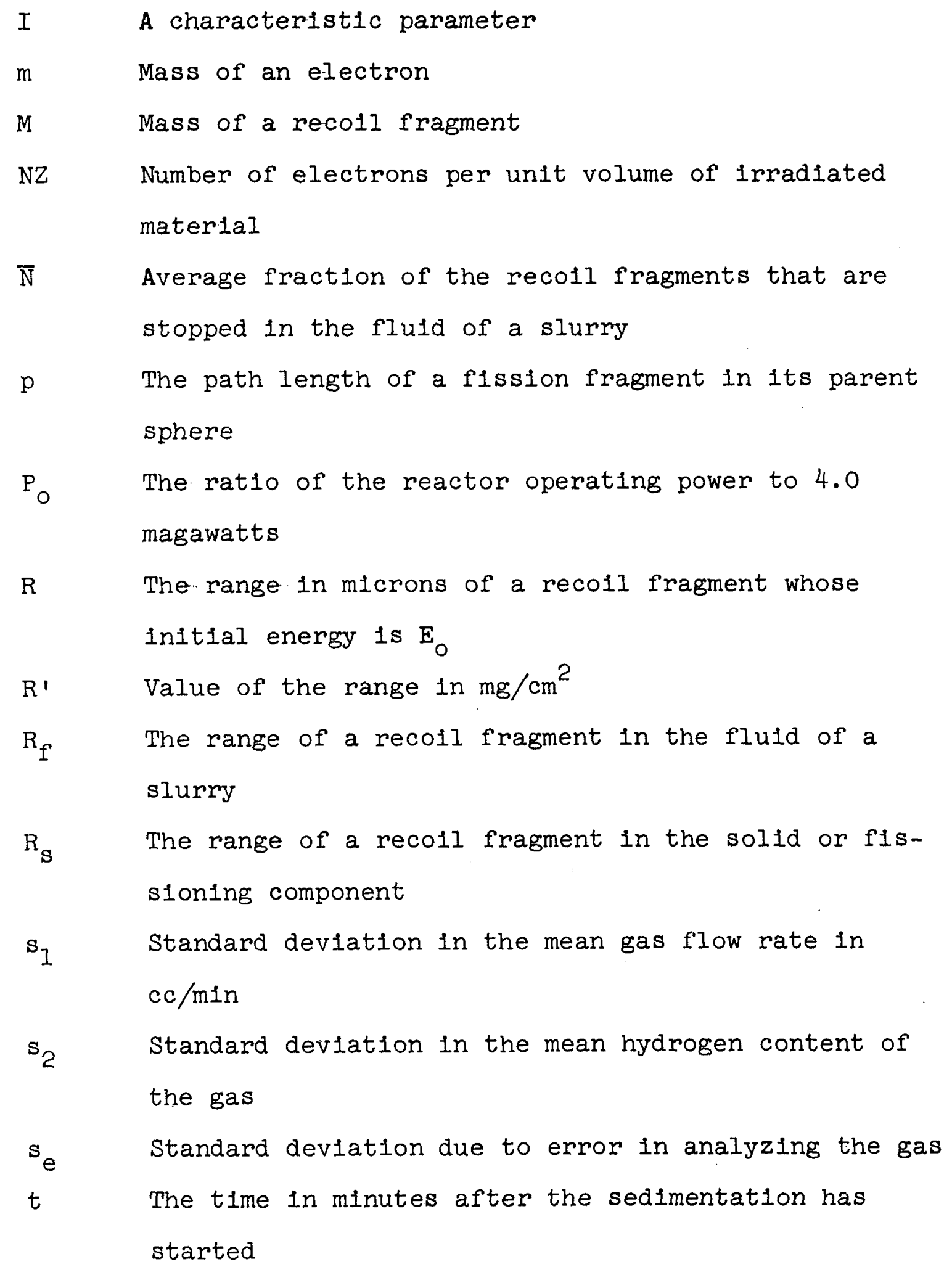




$\begin{array}{ll}v & \text { Settling velocity of a spherical particle in } \mathrm{cm} / \mathrm{sec} \\ \mathrm{w}_{1} & \text { Weight of liquid in the slurry in grams } \\ \mathrm{x} & \text { Distance that a fission fragment has travelled in } \\ \text { microns } & \text { Mean gas flow rate under standard conditions in } \\ \bar{x}_{1} & \text { cc/min } \\ \bar{x}_{2} & \text { Mean hydrogen content of the samples collected dur- } \\ \mathrm{Z} & \text { ing a run } \\ \mathrm{Z}_{\mathrm{R}} & \text { Atomic number } \\ \eta & \text { Viscosity in poises } \\ \mathrm{P} & \text { Density of the solid in } \mathrm{g} / \mathrm{cc} \\ \mathrm{P}_{1} & \text { Density of the liquid in } \mathrm{g} / \mathrm{cc}\end{array}$


APPENDIX I - Data 


\section{PRIMARY DATA}

Experimental conditions

Table 11 gives the inftial conditions for each of the reactor irradiations. The weights of solld and liquid for each mun are the initial quantities placed in the slurry vessel, except for the Iiquid in Run 7. Water splashed out of the cell at the start of the mun; therefore, the weight of water at the end of the mun is given in the table. During this series of experiments, the reactor operating power was increased from 3.8 to 4.0 megawatts. The pressure of the gas in the soap-film flow meter relative to room pressure was measured with the manometer. Room pressure and temperature are also given in the table. Observations

The data which were recorded during the run are given In Table 12 as a function of the irradiation time. The flow rate is shown as the length of time taken for the soap fllm to be displaced by $10 \mathrm{cc}$. of gas. The figure for the total counts represents the number of 1 Mev. electrons ( 3 per cent window-width on the single channel analyzer) reaching the detector, not corrected for dead-time. In addition to these observations, the time at which the gas samples were collected is also shown in the table. Gas analyses

Each of the samples of gas collected during the run was analyzed on the gas chromatograph within a few days 
TABLE 11

EXPERIMENTAL CONDITIONS

\begin{tabular}{|c|c|c|c|c|c|c|c|c|}
\hline Run & Solid & $\begin{array}{c}\text { Weight } \\
\text { gm. }\end{array}$ & Liquid & $\begin{array}{c}\text { Welght } \\
\text { gm. }\end{array}$ & $\begin{array}{c}\text { Power } \\
\text { Mw. }\end{array}$ & $\begin{array}{c}\text { Barometer } \\
\mathrm{mm} . \mathrm{Hg}\end{array}$ & $\begin{array}{c}\text { Manometer } \\
\mathrm{cm} \cdot \mathrm{H}_{2} \mathrm{O}\end{array}$ & Temperature \\
\hline 4 & $\cdot \cdot \cdot$ & • $\cdot \cdot$ & $50 \mathrm{mM} \mathrm{KI}$ & 10.1 & 3.8 & 752.7 & -8 & 26.3 \\
\hline 5 & . . & . . . & $10 \mathrm{mM} \mathrm{U}$ & 9.55 & 3.8 & 757.1 & -13 & 26.6 \\
\hline 6 & - • & $\cdot \cdot \cdot \cdot$ & $50 \mathrm{mM} \mathrm{U}$ & 9.6 & 3.8 & 750.3 & -13 & 26.8 \\
\hline 7 & - $\cdot$ & . $\cdot \cdot$ & $\mathrm{H}_{2} \mathrm{O}$ & 5.2 & 3.8 & 751.8 & -13 & 27.0 \\
\hline 8 & . . . & . . . . & $10 \mathrm{mM} \mathrm{U}$ & 9.55 & 3.8 & 750.0 & -13 & 25.8 \\
\hline 9 & Large & 5.0026 & $\mathrm{H}_{2} \mathrm{O}$ & 8.9 & 4.0 & 745.0 & -13 & 25.2 \\
\hline 10 & Large & 4.9065 & $\mathrm{H}_{2} \mathrm{O}$ & 8.85 & 4.0 & 743.4 & -13 & 26.8 \\
\hline 11 & Large & 10.4979 & $\mathrm{H}_{2} \mathrm{O}$ & 8.4 & 4.0 & 753.0 & -13 & 26.0 \\
\hline 12 & Large & 19.5436 & $\mathrm{H}_{2} \mathrm{O}$ & 7.4 & 4.0 & 748.0 & -13 & 26.0 \\
\hline 13 & Medium & 5.1122 & $\mathrm{H}_{2} \mathrm{O}$ & 8.9 & 4.0 & 743.0 & -13 & 26.0 \\
\hline 14 & Medium & 10.6144 & $\mathrm{H}_{2} \mathrm{O}$ & 8.2 & 4.0 & 745.3 & -13 & $27 \cdot 5$ \\
\hline 15 & Large & 10.1313 & $\mathrm{H}_{2} \mathrm{O}$ & 7.8 & 4.0 & 749.6 & -13 & 27.1 \\
\hline 16 & Small & 2.7021 & $\mathrm{H}_{2} \mathrm{O}$ & 8.7 & 4.0 & 749.4 & -13 & $27 \cdot 7$ \\
\hline 17 & Fine & 3.1650 & $\mathrm{H}_{2} \mathrm{O}$ & 8.5 & 4.0 & 744.8 & -13 & 28.0 \\
\hline 18 & Large & 10.6892 & $\mathrm{H}_{2} \mathrm{O}$ & 8.1 & 4.0 & 742.5 & -13 & $27 \cdot 3$ \\
\hline 19 & Small & 5.0167 & $\mathrm{H}_{2} \mathrm{O}$ & 8.4 & 4.0 & 744.9 & -13 & 28.0 \\
\hline 20 & Fine & 10.0787 & $\mathrm{H}_{2} \mathrm{O}$ & $7 \cdot 9$ & 4.0 & 735.2 & -13 & $27 \cdot 1$ \\
\hline 21 & $\cdot \cdot$ & $\cdot \cdot \cdot \cdot$ & $\mathrm{H}_{2} \mathrm{O}$ & 8.7 & 4.0 & 737.4 & -13 & $27 \cdot 7$ \\
\hline
\end{tabular}


TABLE 12

OBSERVATIONS

Run 4

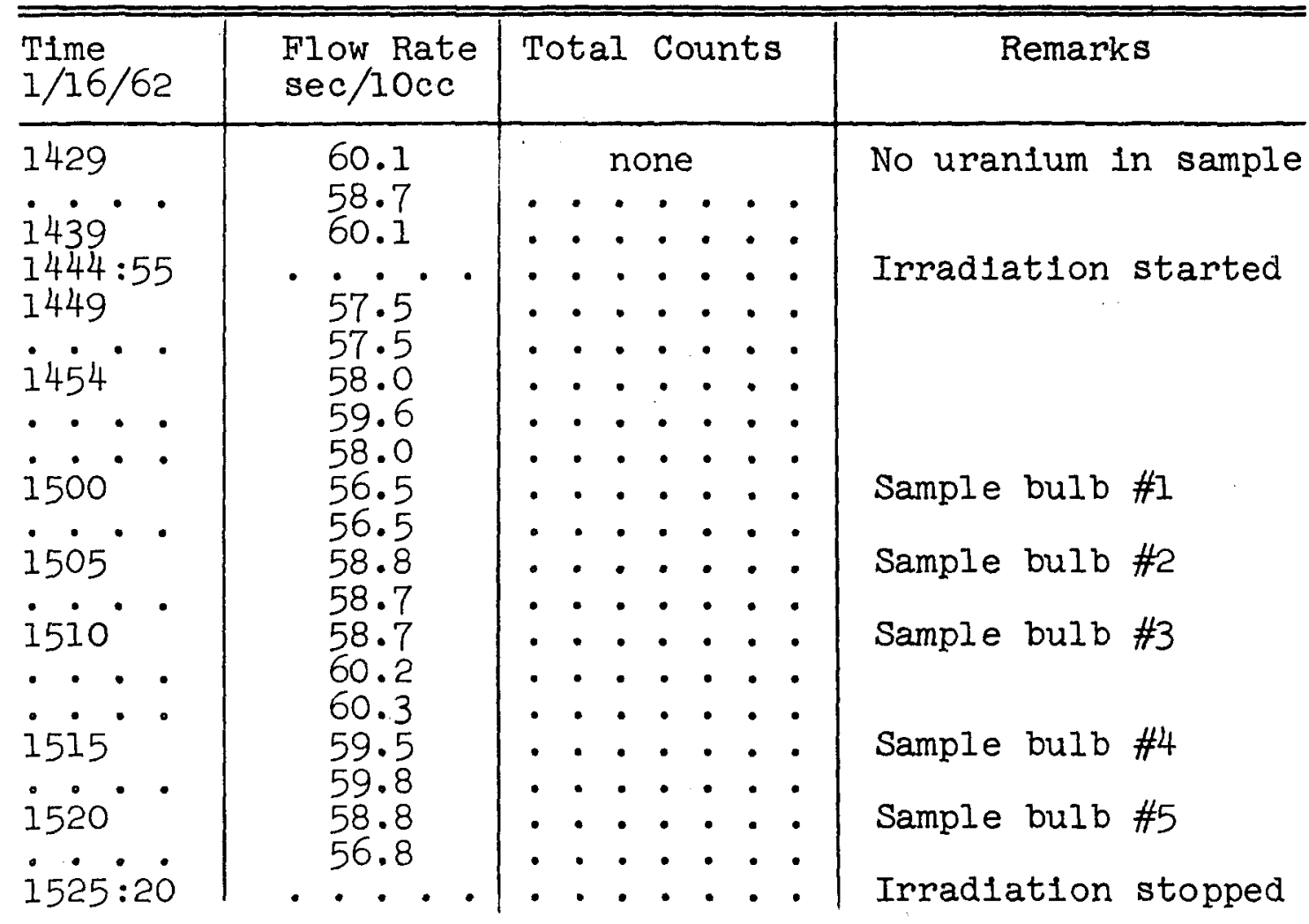

contd. 
TABLE 12 (contd.)

Run 5

\begin{tabular}{|c|c|c|c|}
\hline $\begin{array}{l}\text { Time } \\
1 / 18 / 62\end{array}$ & $\begin{array}{l}\text { Flow Rate } \\
\text { sec/locc }\end{array}$ & Total Counts & Remarks \\
\hline 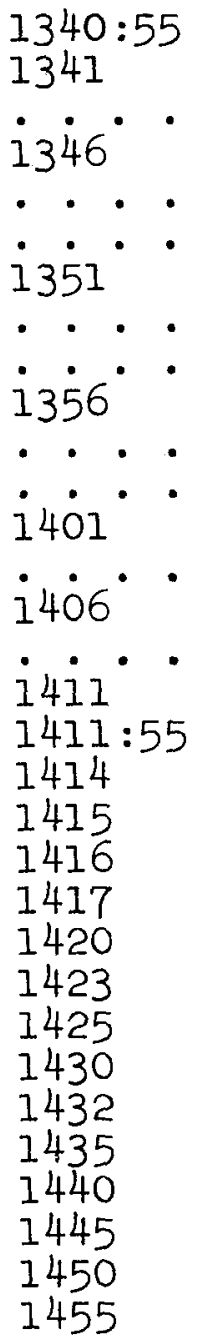 & 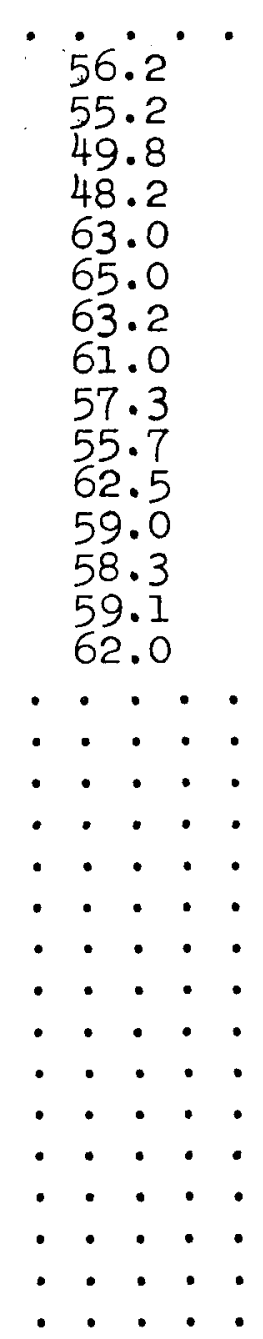 & 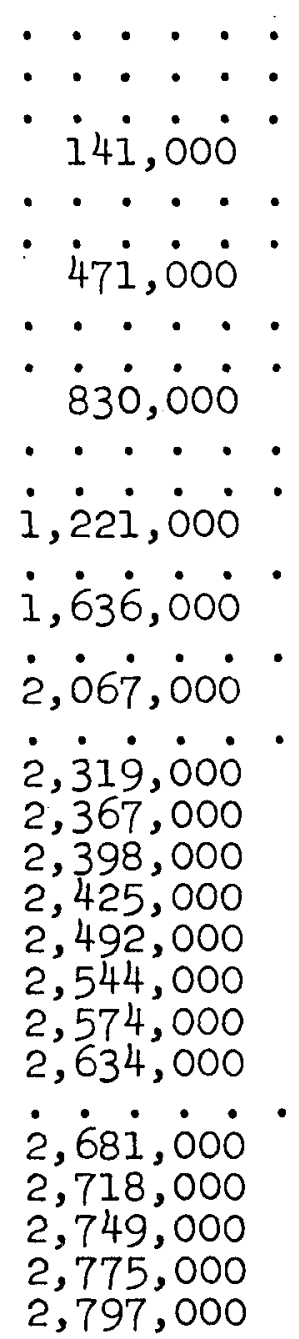 & $\begin{array}{l}\text { Sample bulb \#l } \\
\text { Sample bulb \#2 } \\
\text { Sample bulb \#3 } \\
\text { Irradiation stopped }\end{array}$ \\
\hline
\end{tabular}


TABLE 12 (contd.)

Run 6

\begin{tabular}{|c|c|c|c|}
\hline $\begin{array}{l}\text { Time } \\
1 / 19 / 62\end{array}$ & $\begin{array}{l}\text { Flow Rate } \\
\text { sec/locc }\end{array}$ & Total Counts & Remarks \\
\hline 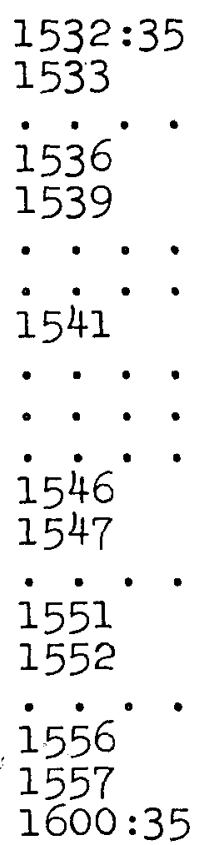 & 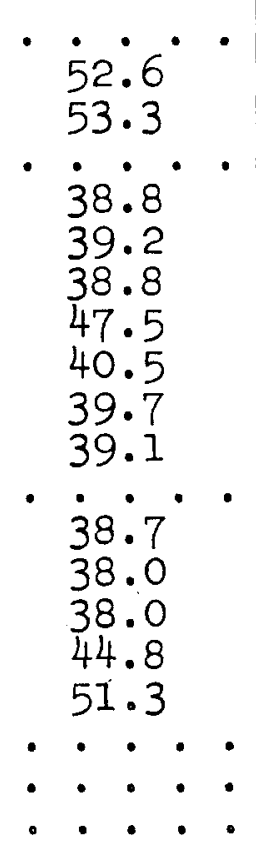 & 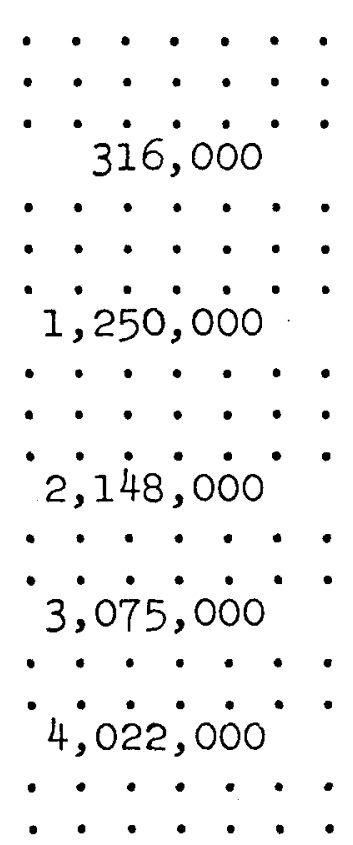 & $\begin{array}{l}\text { Sample bulb \#5 } \\
\text { Sample bulb \#6 } \\
\text { Sample bulb \#7 } \\
\text { Irradiation stopped }\end{array}$ \\
\hline
\end{tabular}


TABLE 12 (contd.)

Run 7

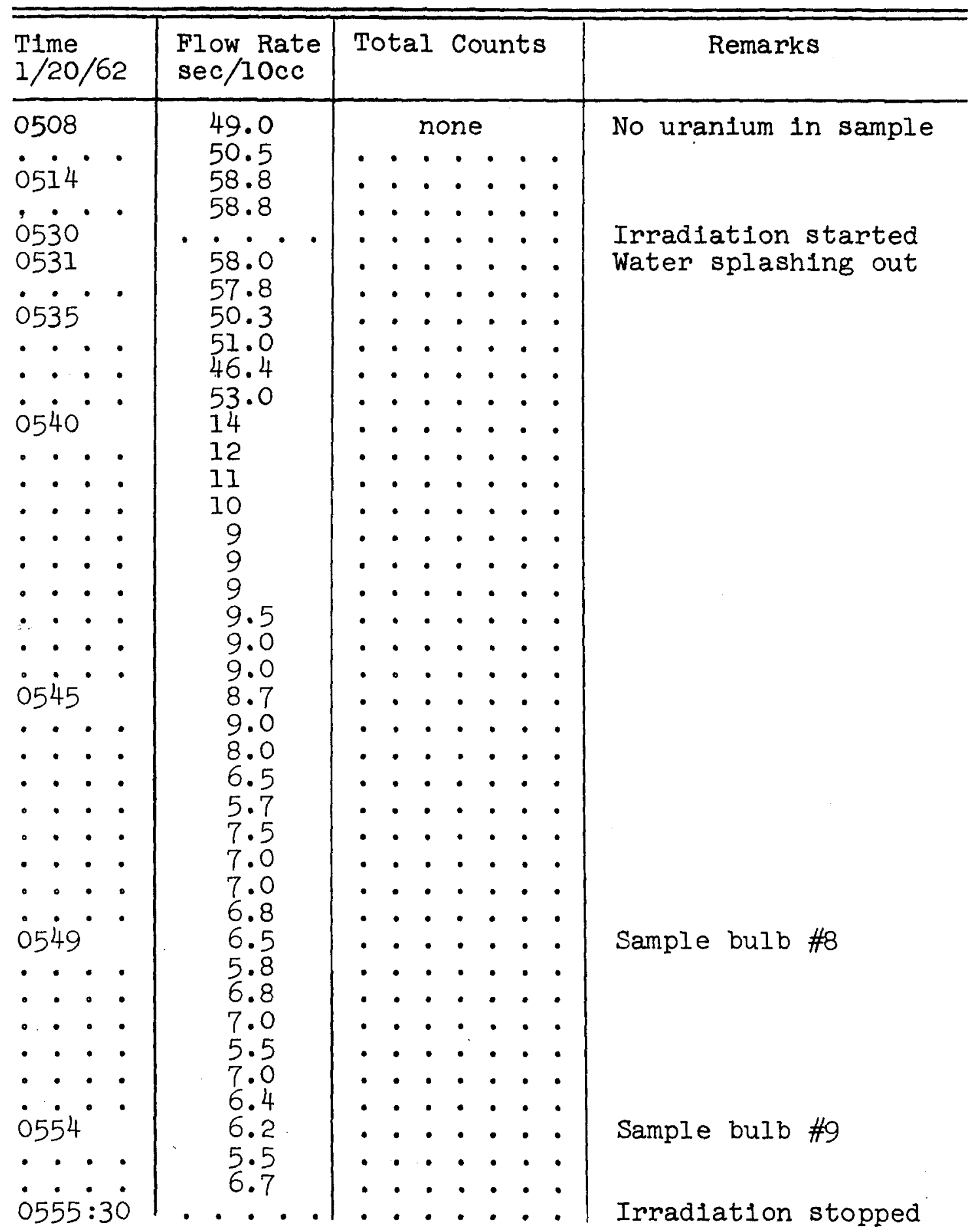


TABLE 12 (contd.)

Run 8

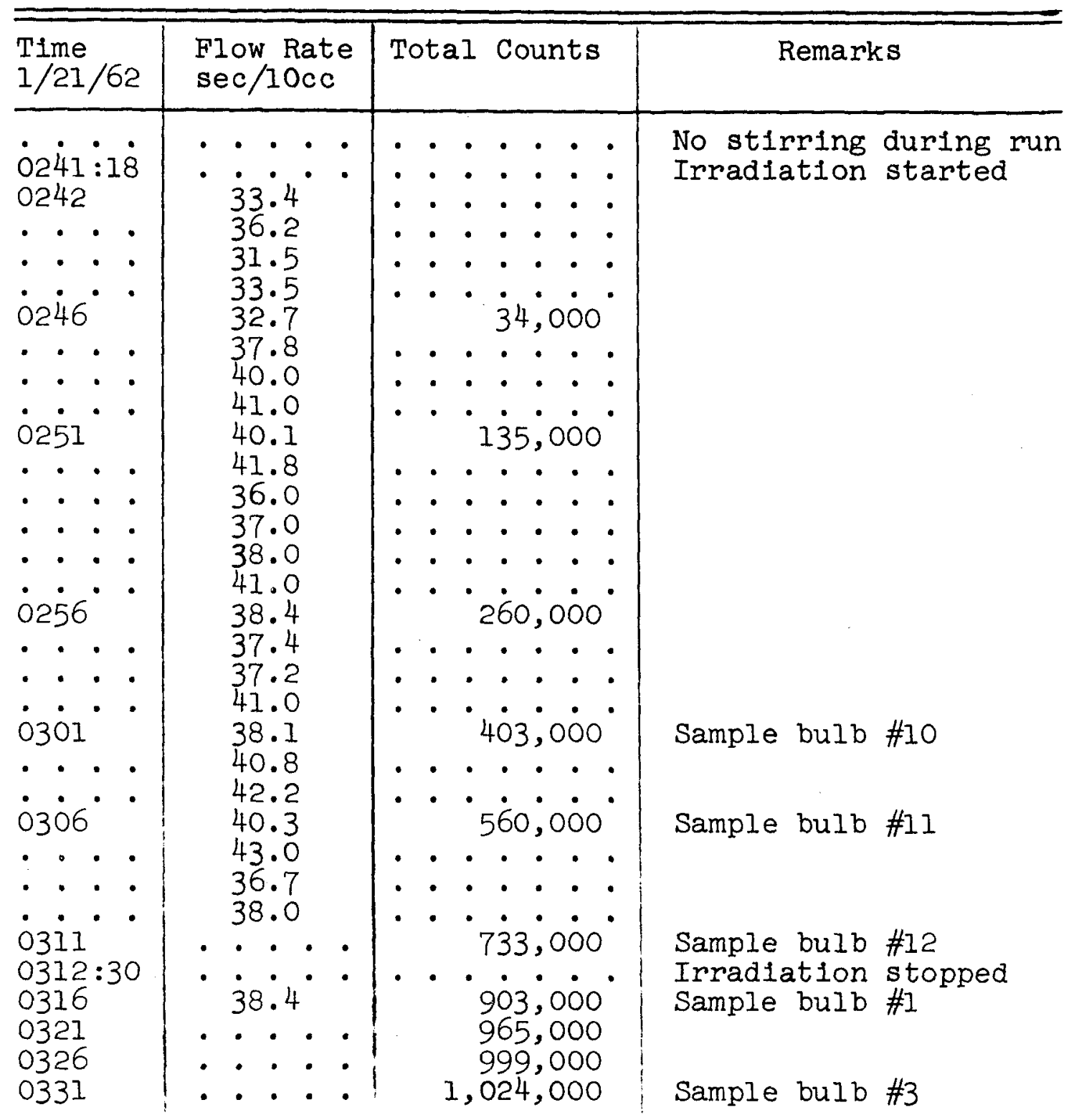


TABLE 12 (contd.)

Run 9

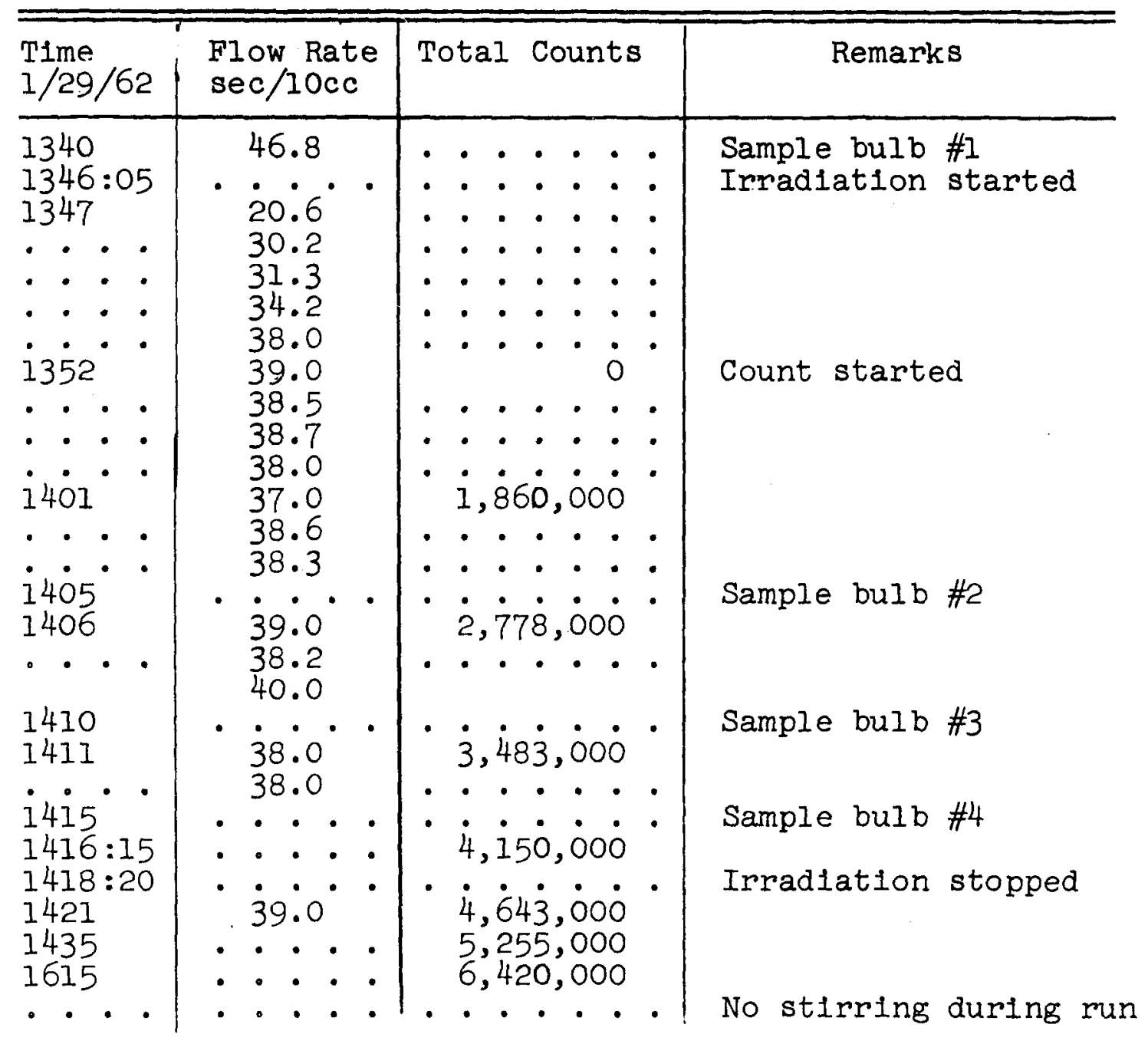


TABLE 12 (contd.)

Run 10

\begin{tabular}{|c|c|c|c|}
\hline $\begin{array}{l}\text { Time } \\
1 / 29 / 62\end{array}$ & $\begin{array}{l}\text { Flow Rate } \\
\text { sec/10cc. }\end{array}$ & Total Counts & Remarks \\
\hline 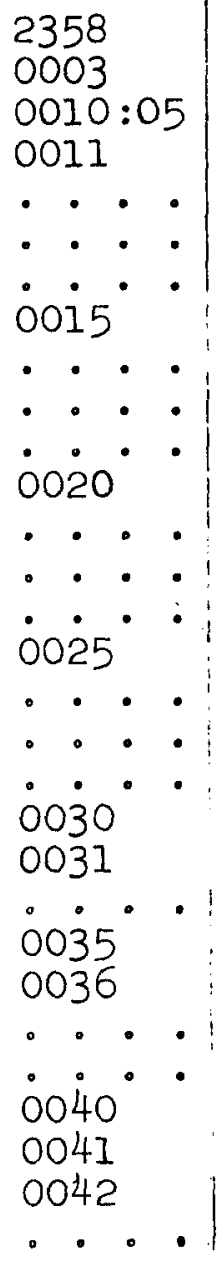 & 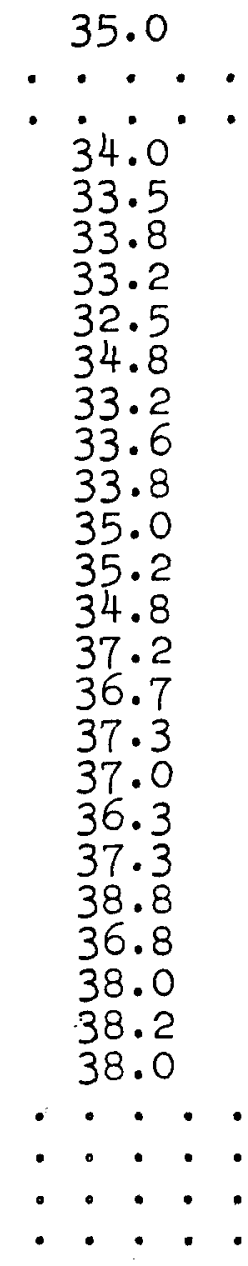 & 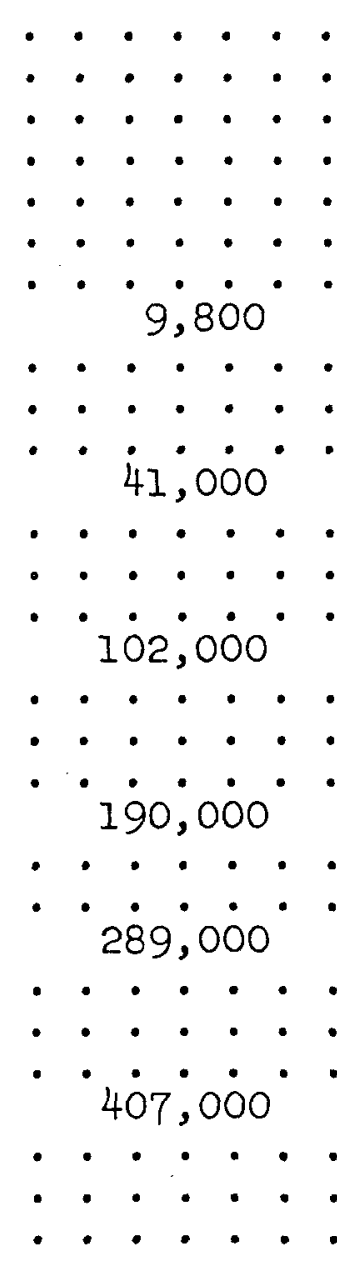 & $\begin{array}{l}\text { Sample bulb \#6 } \\
\text { Sample bulb \#7 } \\
\text { Sample bulb \#8 } \\
\text { Irradiation stopped } \\
\text { No stirring during ru }\end{array}$ \\
\hline
\end{tabular}


TABLE 12 (contd.)

Run 11

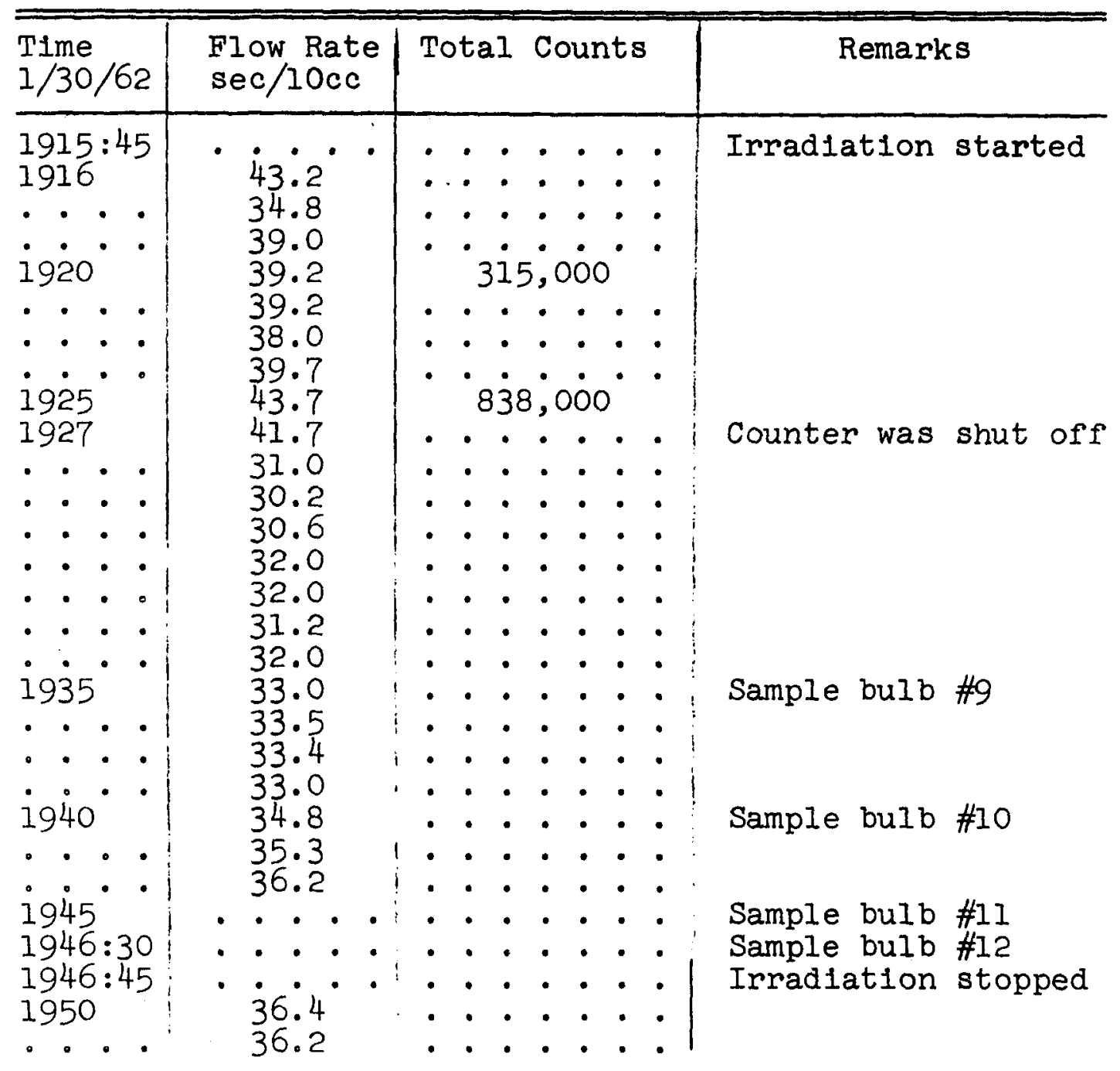


TABLE 12 (contd.)

Run 12

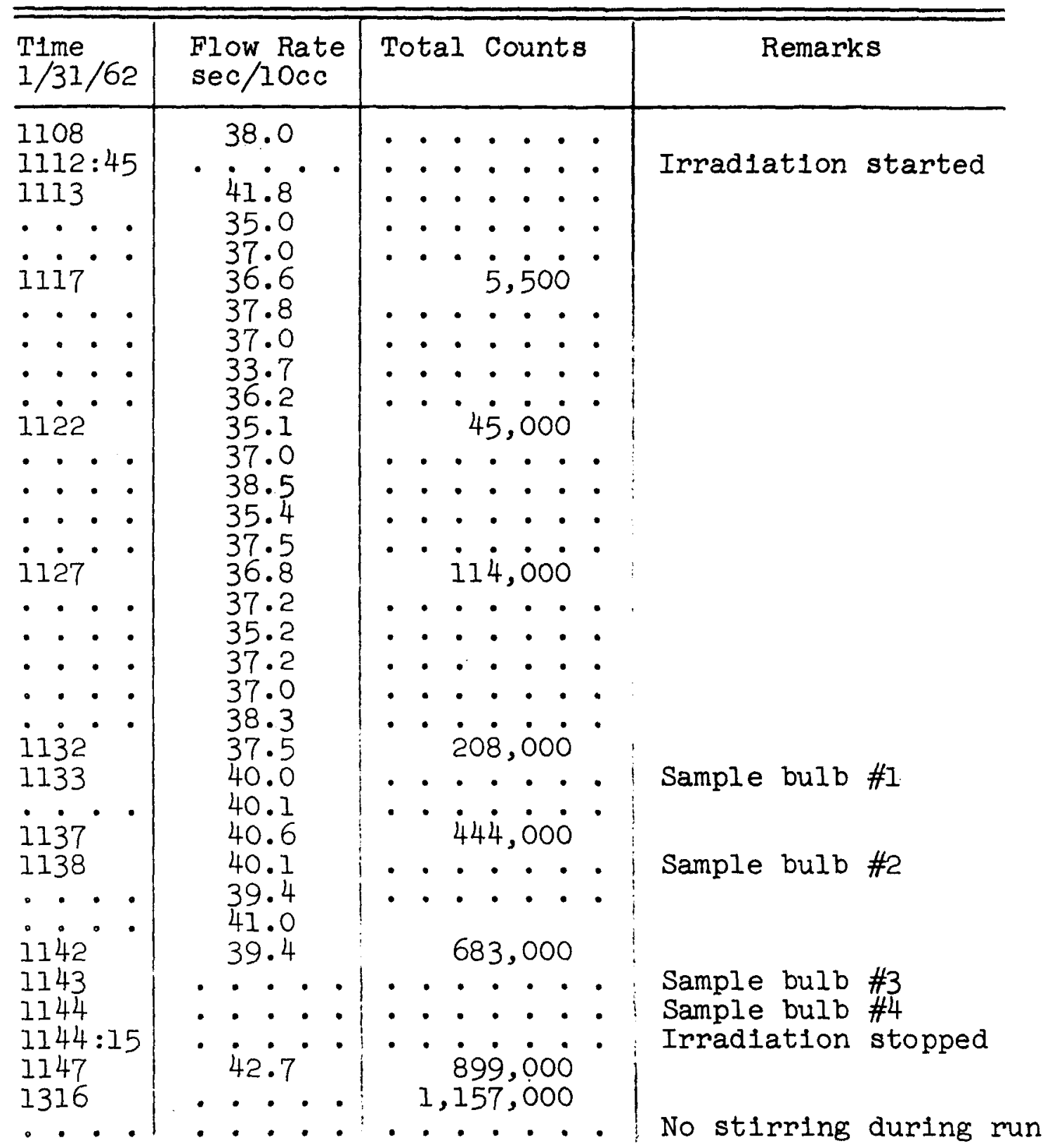


TABLE 12 (contd.)

Run 13

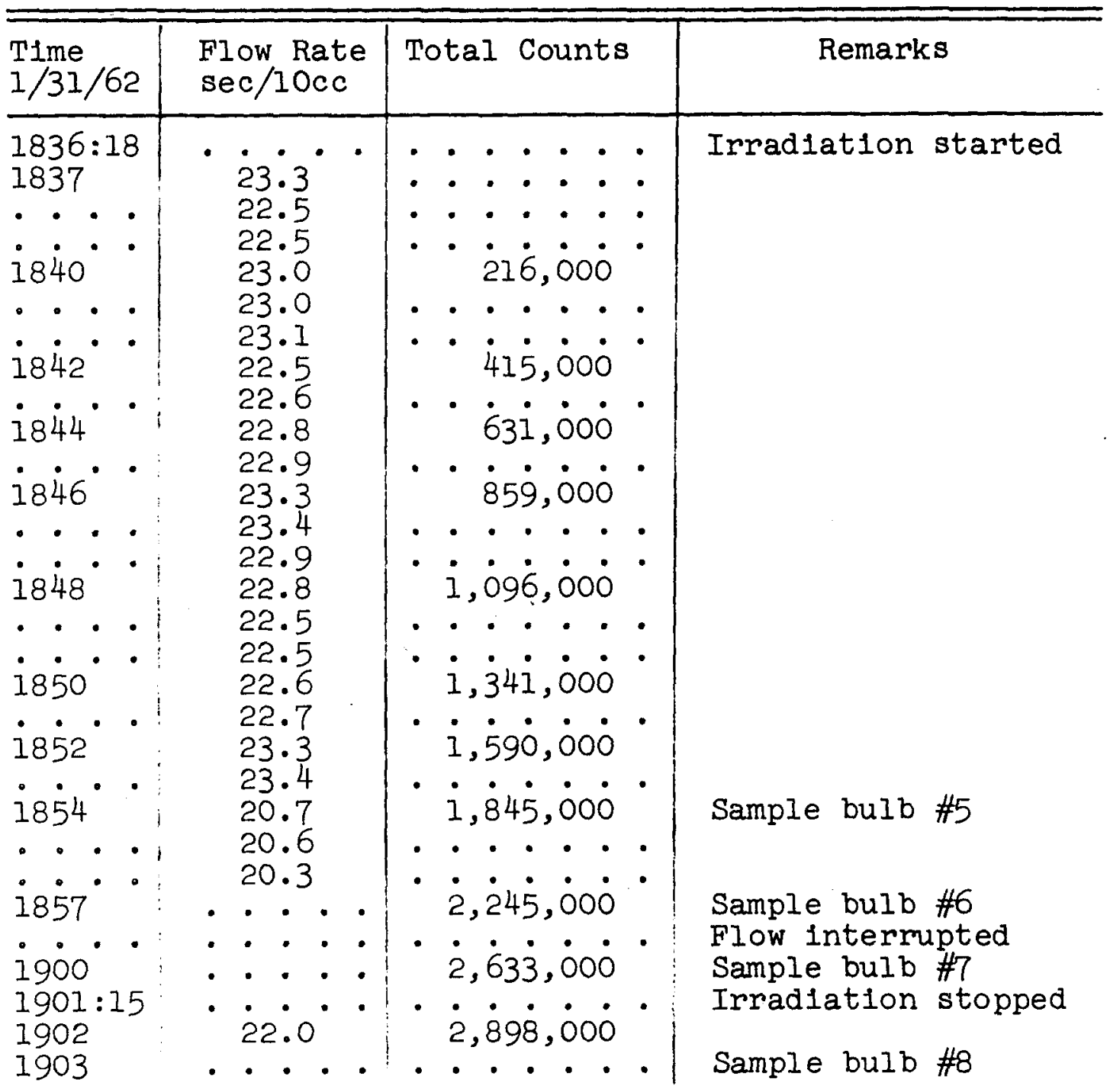


TABLE 12 (contd.)

Run 14

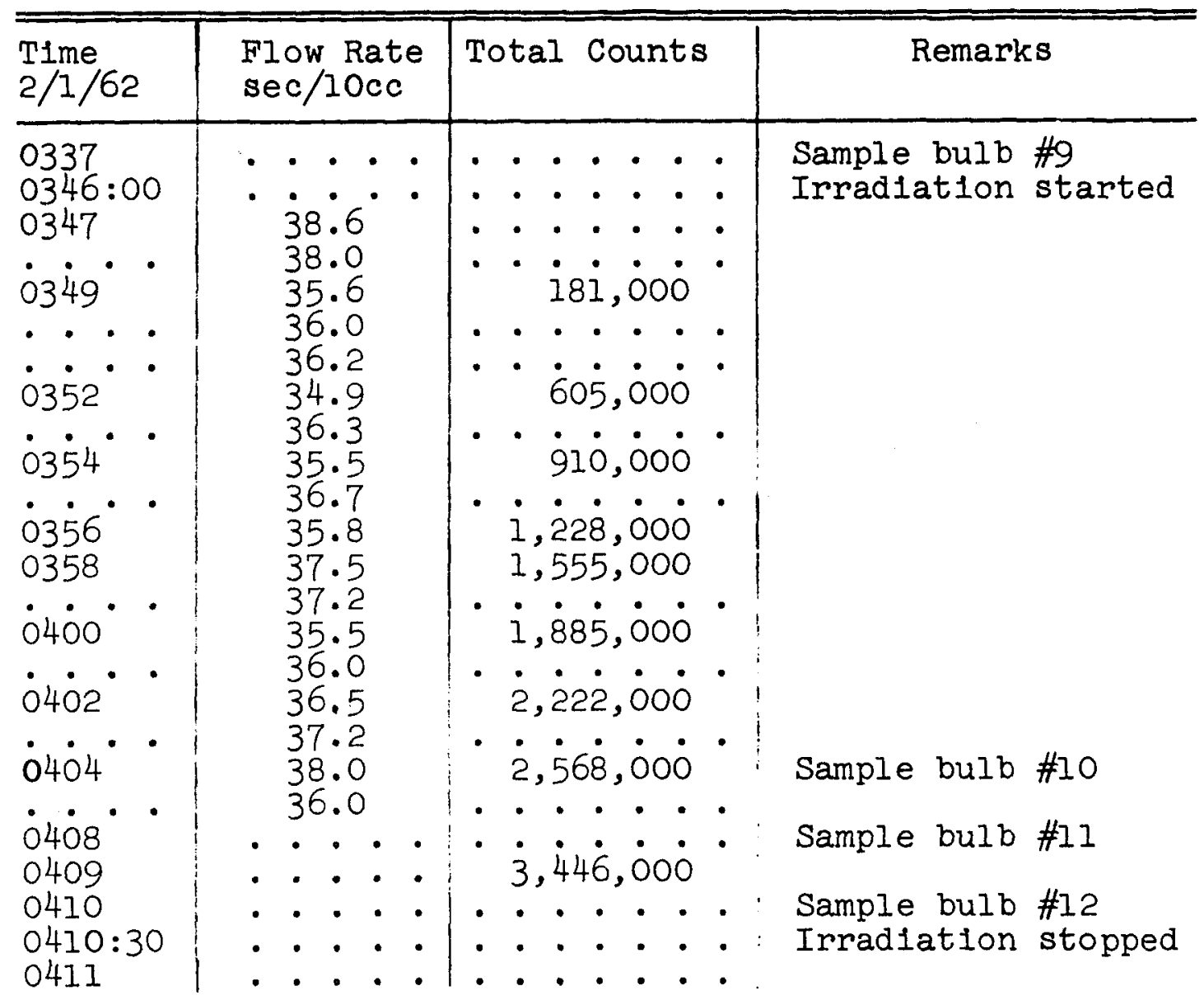


TABLE 12 (contd.)

Run 15

\begin{tabular}{|c|c|c|c|}
\hline $\begin{array}{l}\text { Time } \\
2 / 1 / 62\end{array}$ & $\begin{array}{l}\text { Flow Rate } \\
\text { sec/10cc }\end{array}$ & Total Counts & Remarks \\
\hline 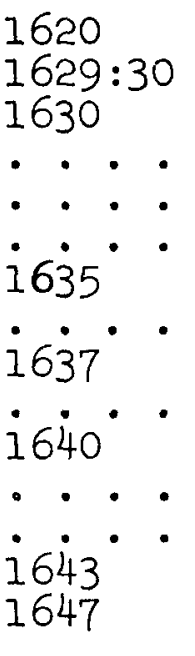 & $\begin{array}{l}\cdot: \cdot \dot{0} \\
35.7 \\
35.3 \\
35.3 \\
34.5 \\
37.3 \\
34.5 \\
34.6 \\
35.0 \\
35.0 \\
35.0 \\
35.0 \\
.0 \\
35.3 \\
34.8\end{array}$ & 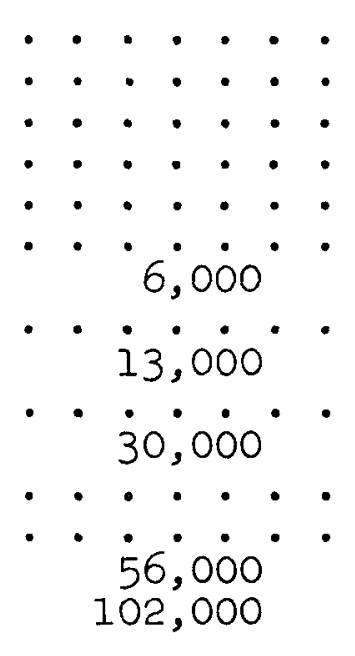 & $\begin{array}{l}\text { Sample bulb \#1 } \\
\text { Irradiation started }\end{array}$ \\
\hline $\begin{array}{l}1650^{\circ} \\
1653 \\
1656 \\
1656: 40 \\
1658 \\
1701\end{array}$ & $\begin{array}{l}34.8 \\
36.5 \\
\therefore:\end{array}$ & $\begin{array}{l}149,000^{\circ} \\
199,000 \\
259,000 \\
.315,000 \\
355,000\end{array}$ & $\begin{array}{l}\text { Sample bulb \#2 } \\
\text { Sample bulb \#3 } \\
\text { Sample bulb \#4 } \\
\text { Irradiation stopped } \\
\text { No stirring during }\end{array}$ \\
\hline
\end{tabular}


TABLE 12 (contd.)

Run 16

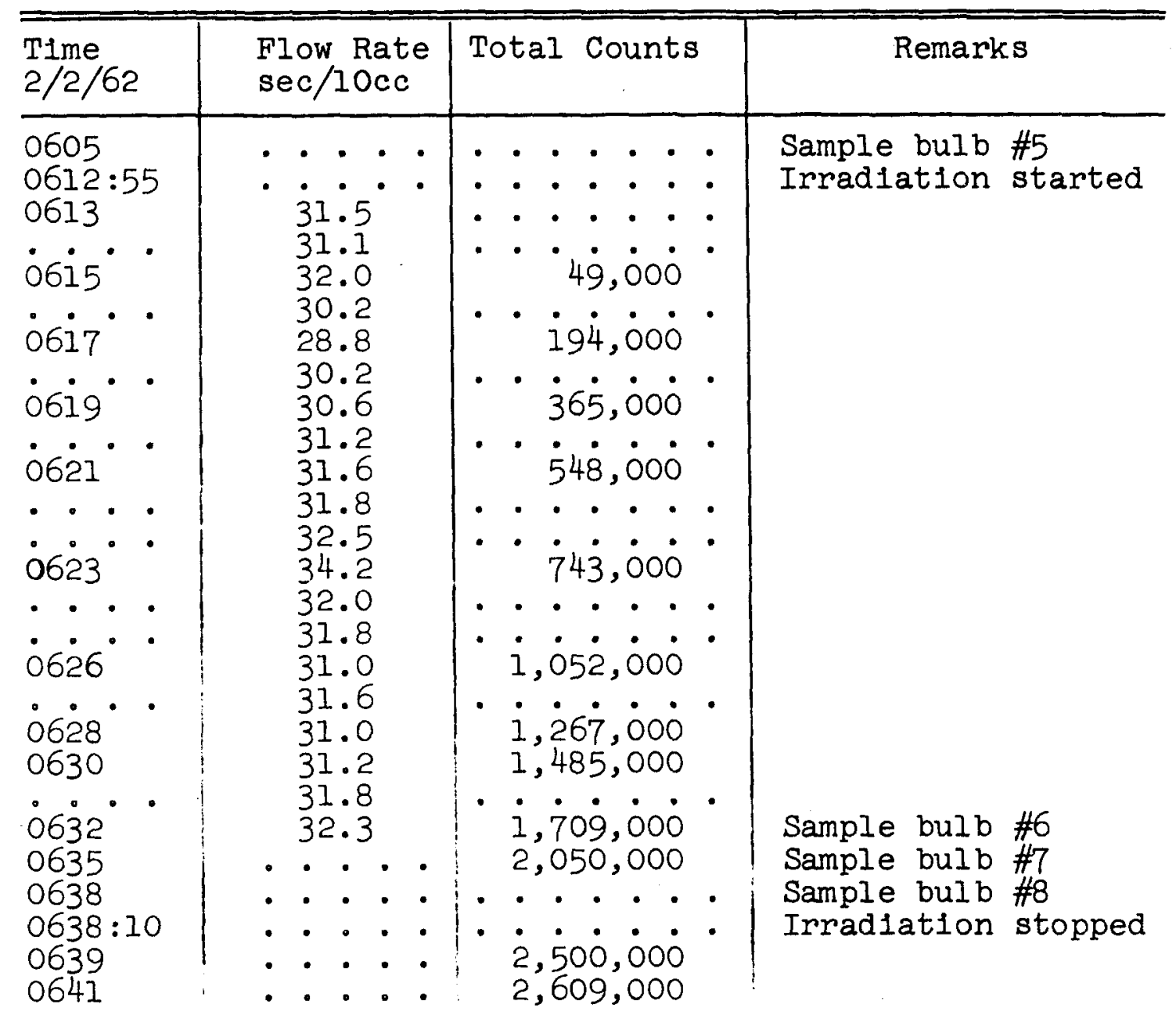


TABLE 12 (contd.)

Run 17

\begin{tabular}{|c|c|c|c|}
\hline $\begin{array}{l}\text { Time } \\
2 / 2 / 62\end{array}$ & $\begin{array}{l}\text { Flow Rate } \\
\mathrm{sec} / 10 \mathrm{cc}\end{array}$ & Total Counts & Remarks \\
\hline 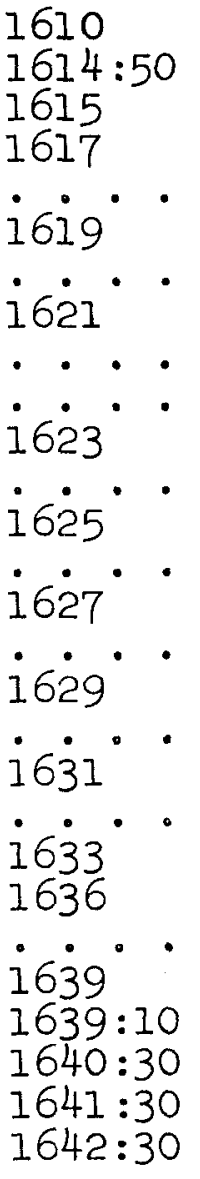 & 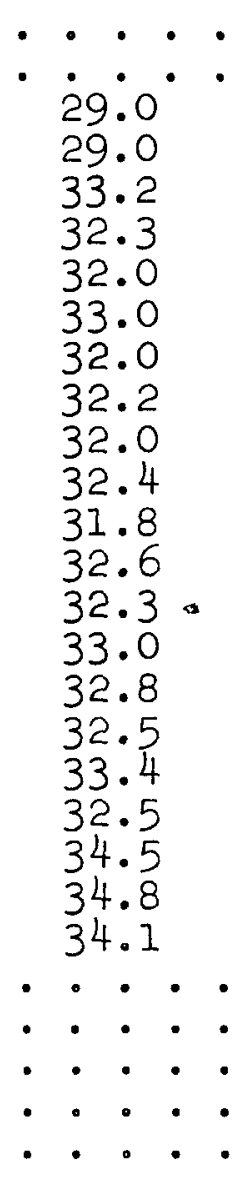 & 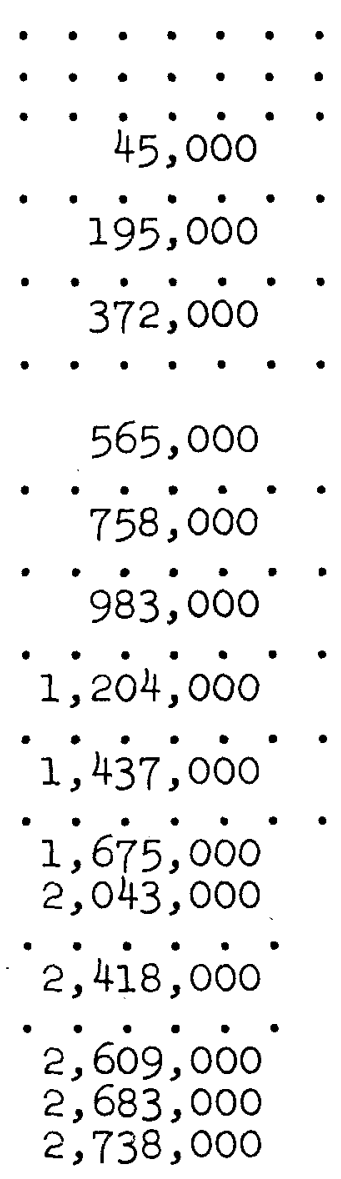 & $\begin{array}{l}\text { Sample bulb \#10 } \\
\text { Sample bulb \#11 } \\
\text { Sample bulb \#12 } \\
\text { Irradiation stopped }\end{array}$ \\
\hline
\end{tabular}


TABLE 12 (contd.)

Run 18

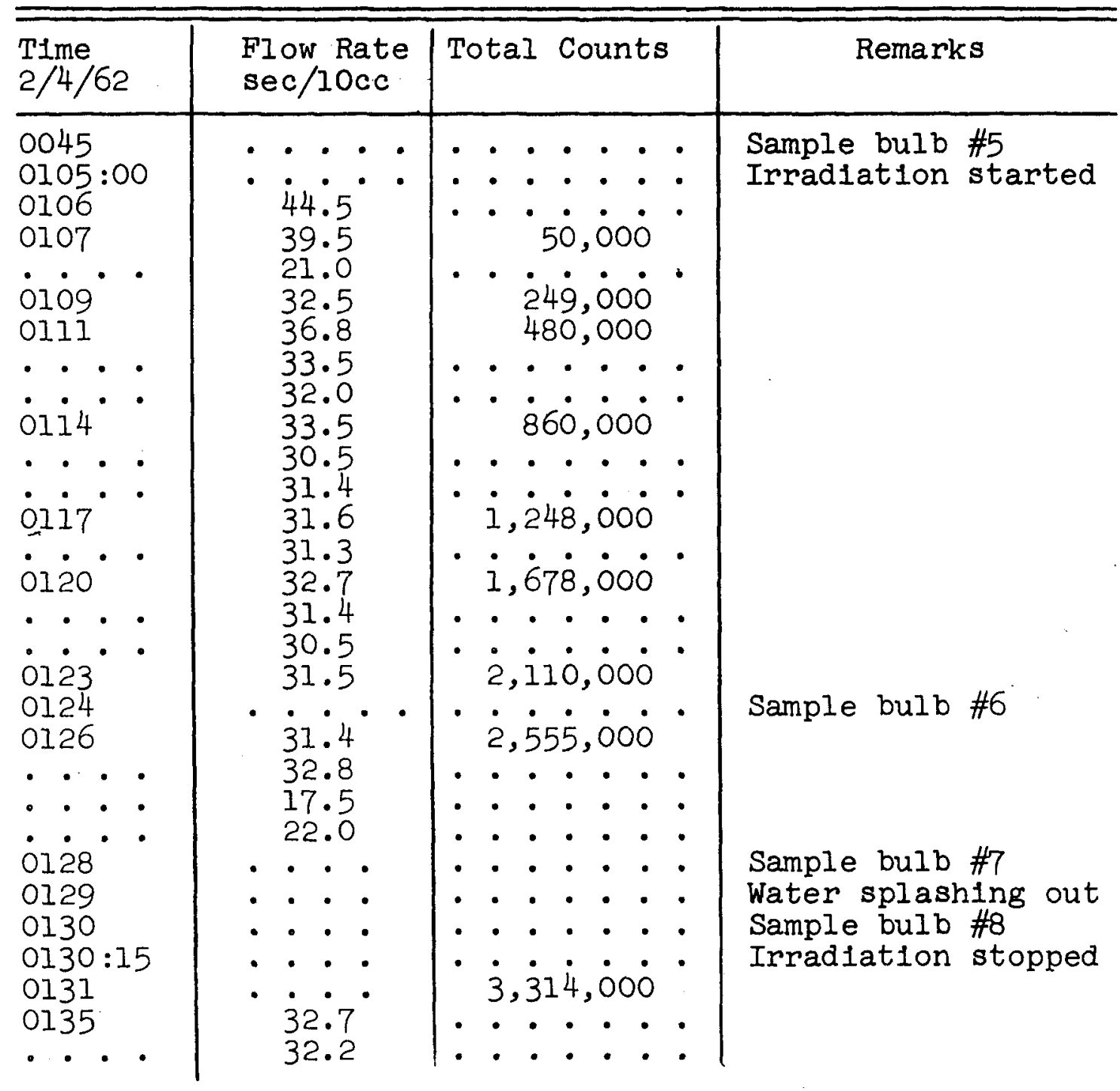


TABLE 12 (contd.)

Run 19

\begin{tabular}{|c|c|c|c|}
\hline $\begin{array}{l}1 \mathrm{me} \\
13 / 62\end{array}$ & $\begin{array}{l}\text { Flow Rate } \\
\text { sec/locc }\end{array}$ & Total Counts & Remarks \\
\hline 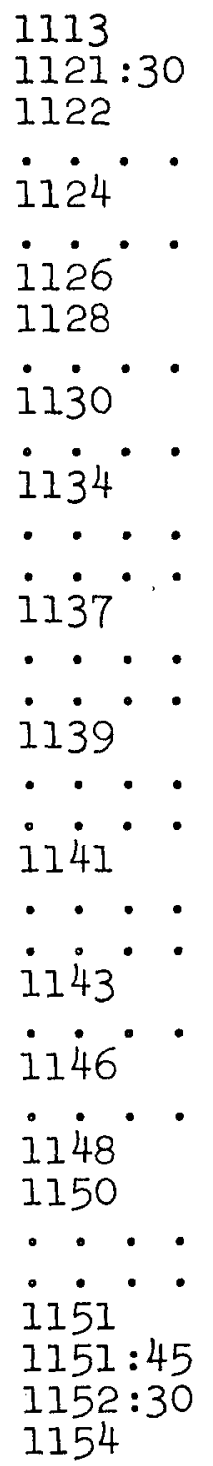 & 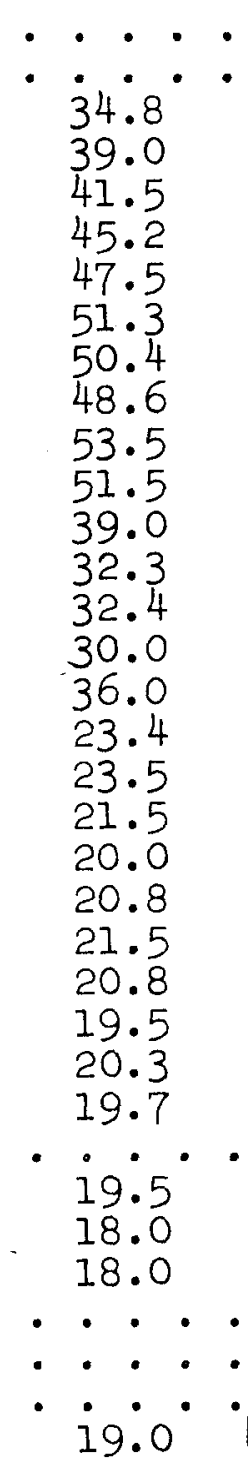 & 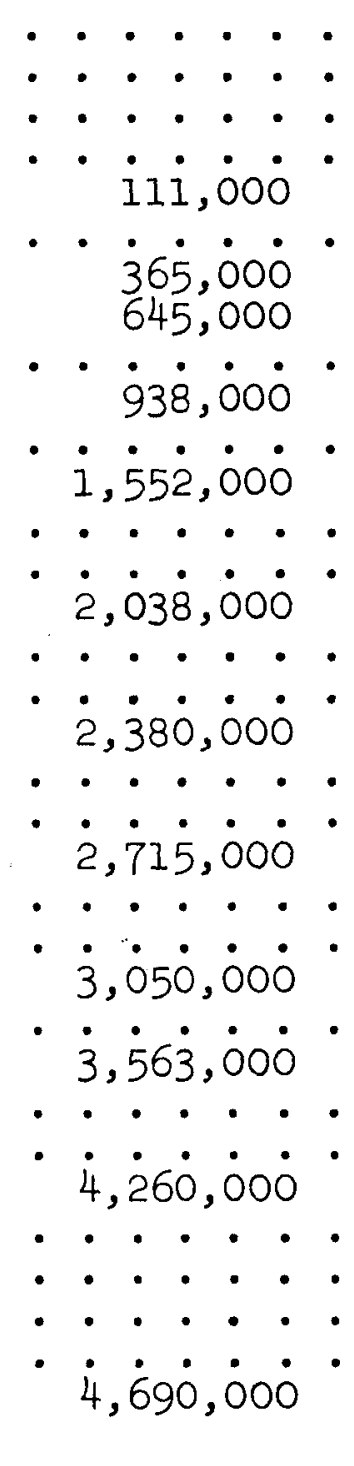 & $\begin{array}{l}\text { Sample bulb \#2 } \\
\text { Sample bulb \#3 } \\
\text { Sample bulb \#4 } \\
\text { Irradiation stopped }\end{array}$ \\
\hline
\end{tabular}


TABLE 12 (contd.)

Run 20

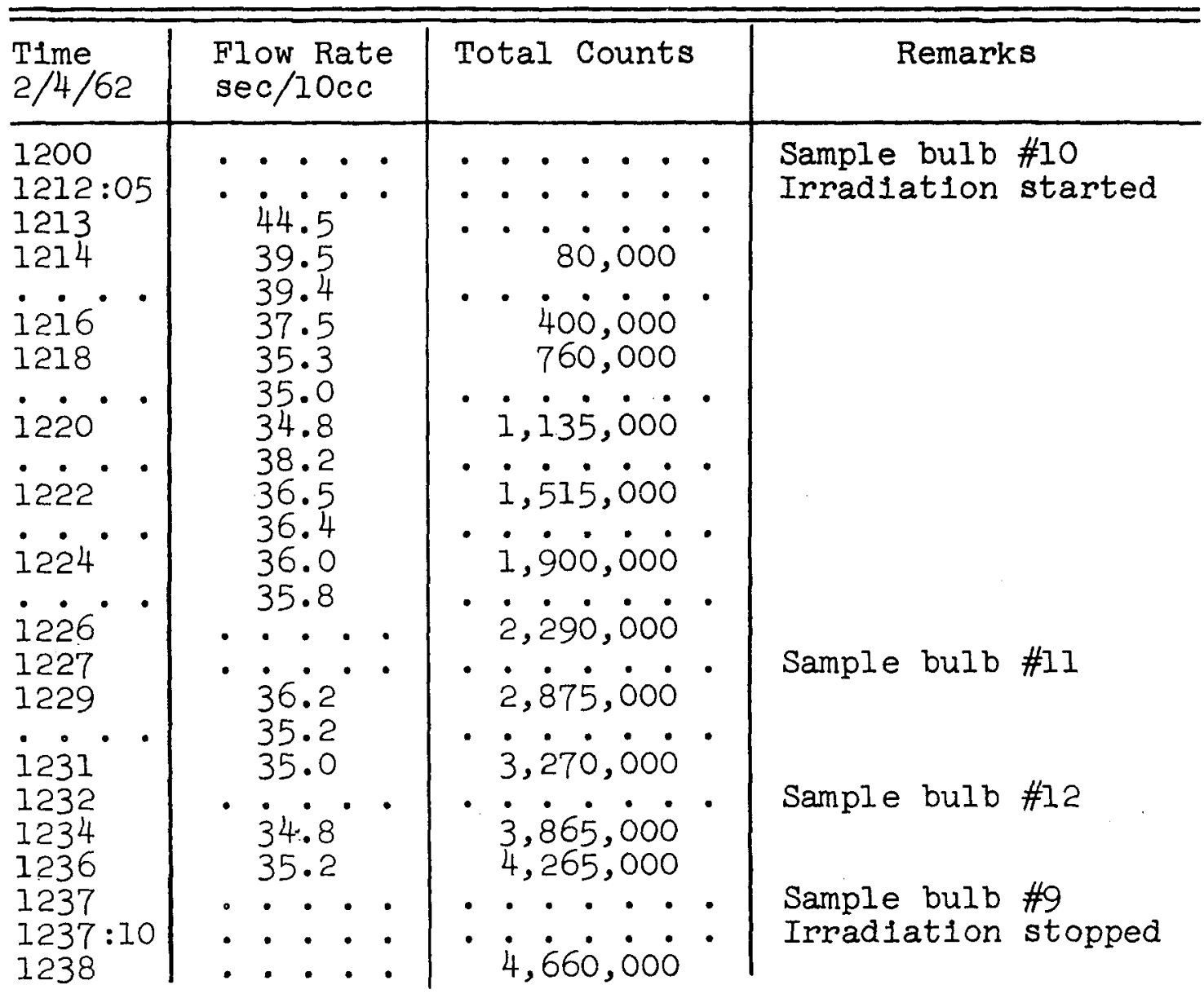


TABLE 12 (contd.)

Run 21

\begin{tabular}{|c|c|c|c|c|c|c|}
\hline $\operatorname{lime}_{15 / 62}$ & $\begin{array}{l}\text { Flow Rate } \\
\text { sec } / 10 \mathrm{cc}\end{array}$ & Tota. & c & ount & & Remarks \\
\hline 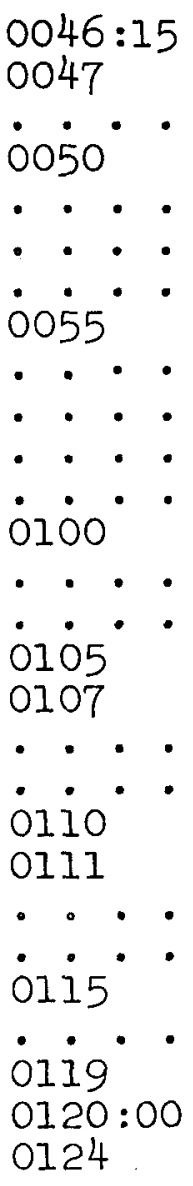 & $\begin{array}{l}44.4 \\
44.0 \\
46.5 \\
42.0 \\
44.0 \\
43.0 \\
43.0 \\
41.6 \\
45.0 \\
42.2 \\
42.4 \\
42.8 \\
42.2 \\
41.5 \\
.43 .0 \\
43.0 \\
41.5 \\
.40 . \\
43.0 \\
42.0 \\
43.5 \\
42.3 \\
43.3 \\
.0 .0 \\
.0\end{array}$ & 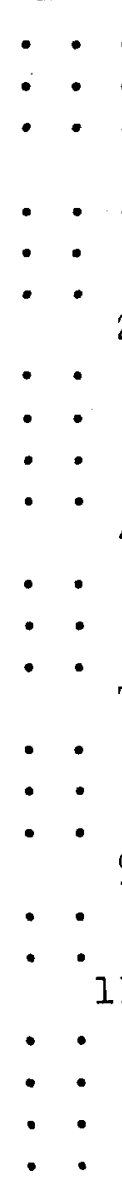 & $\bullet$ & 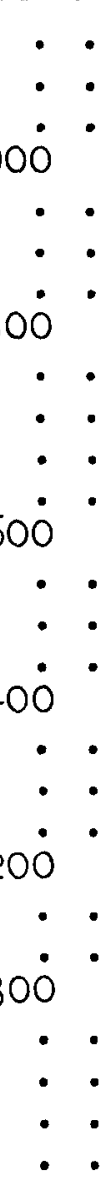 & 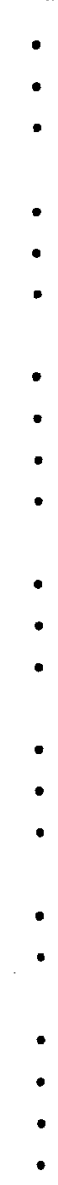 & $\begin{array}{l}\text { Sample bulb \#1 } \\
\text { Sample bulb \#2 } \\
\text { Sample bulb \#3 } \\
\text { Sample bulb \#4 } \\
\text { Sample bulb \#5 } \\
\text { Irradiation stopped } \\
\text { Sample bulb \#6 }\end{array}$ \\
\hline
\end{tabular}


after the mun. These data, as well as that for the cal1bration mixture, are given in Table 13. The quantity of gas which was analyzed is given by the volume and pressure measurements. The actually measured areas under the hydrogen and oxygen peaks are shown along with the factor by which each area had been amplified. From the analysis of the calibration mixture, shown in Figure 27, the sensitivity factor for hydrogen was found to be $0.0379 \mathrm{~mm}$. $\mathrm{H}_{2} / \mathrm{cm}^{2}$ for a $2.0 \mathrm{cc}$. sample during runs 4 through 8 . For the rest of the runs, 9 through 21 , this factor was 0.0423 . The hydrogen content of the samples has been calculated from these sensitivity factors. Since accurate analysis of the oxygen content was not necessary, the sensitivity factor for oxygen was approximated as one-tenth that for hydrogen.

Fission product analyses

Determination of the number of fissions which occurred in the slurry during a mun is shown in Table 14. The first two columns give the fission density for the I1quid and solid respectively, based on the analysis for molybdenum-99. The third column shows that for the liquid based on the amount of cerium-144. Average deviation in these results is less than 5 per cent unless noted in the table. Using these results and the quantities of liquid and solid originally in the cell, the total number of 
TABLE 13

GAS ANALYSES

\begin{tabular}{|c|c|c|c|c|c|c|c|c|c|}
\hline 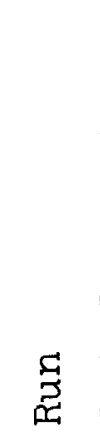 & 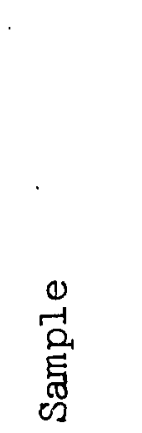 & $\begin{array}{l}\dot{0} \\
\dot{0} \\
\hat{0} \\
\overrightarrow{5} \\
\overrightarrow{0} \\
\overrightarrow{0}\end{array}$ & 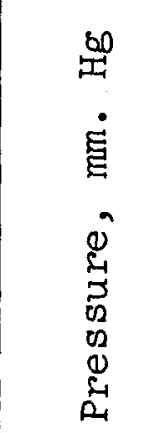 & 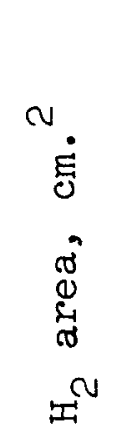 & 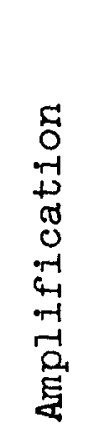 & 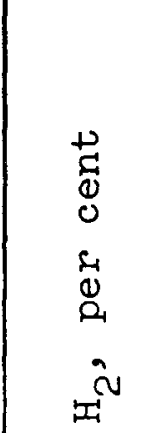 & 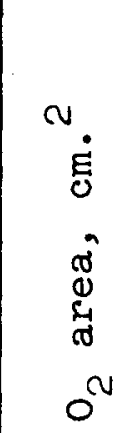 & 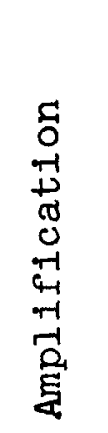 & 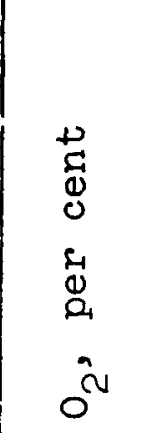 \\
\hline 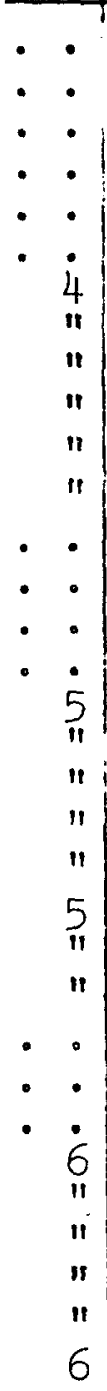 & 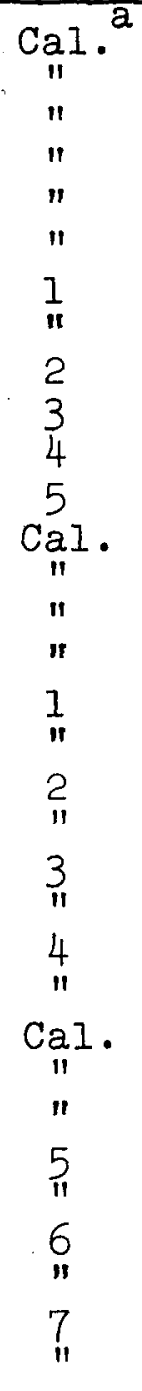 & 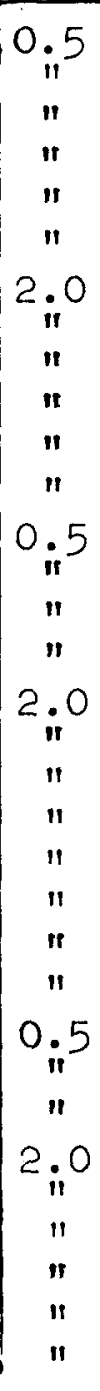 & $\begin{array}{c}62 \\
140.5 \\
72 \\
72 \cdot 5 \\
218 \\
197 \\
435 \\
146 \\
188 \\
158 \\
165 \\
173 \\
55 \cdot 5 \\
219 \cdot 5 \\
157 \cdot 5 \\
126 \\
259 \\
150.5 \\
241 \\
228 \\
167 \\
194 \\
115 \\
299 \\
69 \cdot 5 \\
164 \\
41 \\
214 \\
191 \\
201 \\
114 \\
104 \cdot 5 \\
157\end{array}$ & $\begin{array}{l}18.4 \\
22.7 \\
24.0 \\
23.4 \\
41.0 \\
31.3 \\
48.1 \\
63.1 \\
53.0 \\
57.6 \\
58.5 \\
19.0 \\
37.2 \\
27.4 \\
45.4 \\
48.7 \\
28.0 \\
39.0 \\
35.5 \\
26.5 \\
31.7 \\
5.7 \\
14.2 \\
22.9 \\
22.6 \\
14.4 \\
57.2 \\
52.0 \\
57.9 \\
31.2 \\
36.1 \\
53.9\end{array}$ & $\begin{array}{r}10 \\
5 \\
10 \\
10 \\
5 \\
5 \\
. \quad \\
5 \\
5 \\
5 \\
5 \\
5 \\
10 \\
5 \\
5 \\
10 \\
5 \\
5 \\
5 \\
5 \\
5 \\
5 \\
5 \\
5 \\
10 \\
5 \\
10 \\
5 \\
5 \\
5 \\
5 \\
5 \\
5\end{array}$ & $\begin{array}{c}0.532 \\
" 11 \\
" 1 \\
" 1 \\
0.253 \\
0.253 \\
0.257 \\
0.258 \\
0.268 \\
0.259 \\
0.532 \\
11 \\
11 \\
0.145 \\
0.144 \\
0.125 \\
0.120 \\
0.123 \\
0.126 \\
0.042 \\
0.038 \\
0.532 \\
-11 \\
11 \\
0.205 \\
0.209 \\
0.221 \\
0.211 \\
0.266 \\
0.263\end{array}$ & 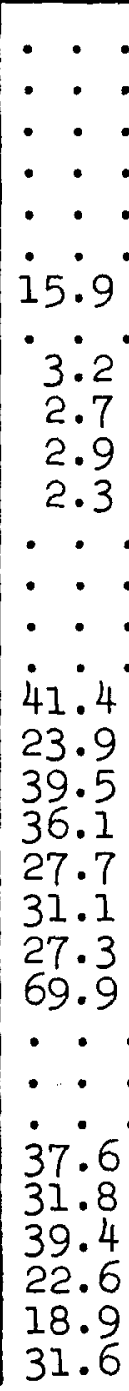 & 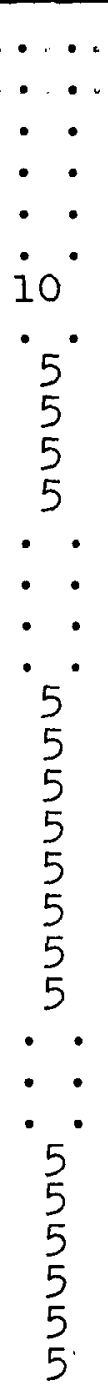 & 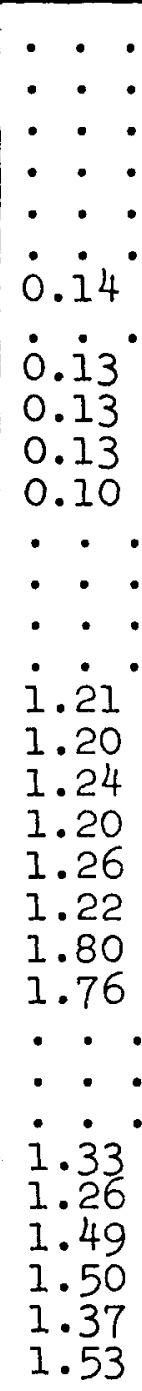 \\
\hline
\end{tabular}


TABLE 13 (contd.)

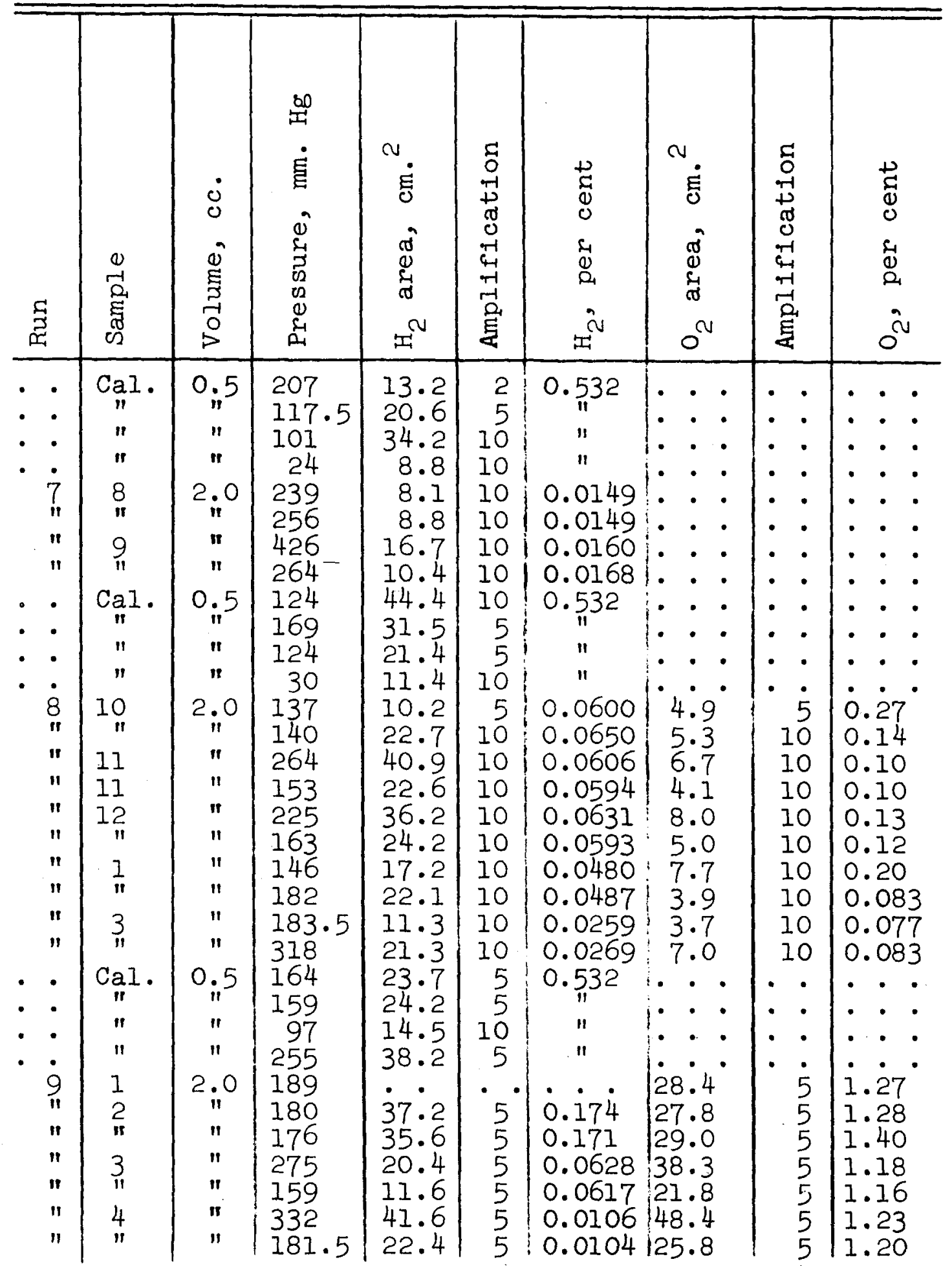


141

TABLE 13 (contd.)

\begin{tabular}{|c|c|c|c|c|c|c|c|c|c|}
\hline 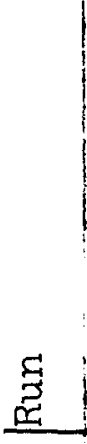 & 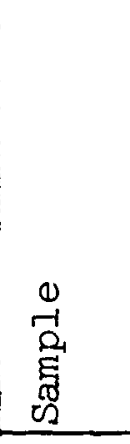 & $\begin{array}{r}\dot{0} \\
0 \\
0 \\
0 \\
\vdots \\
5 \\
\overrightarrow{1} \\
0 \\
\end{array}$ & 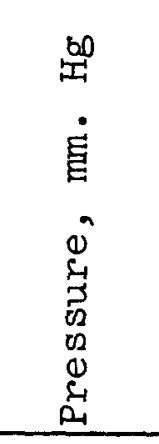 & 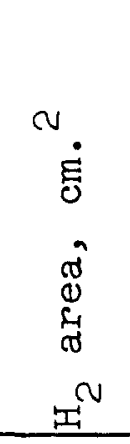 & 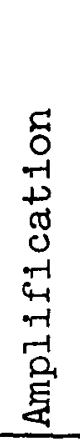 & 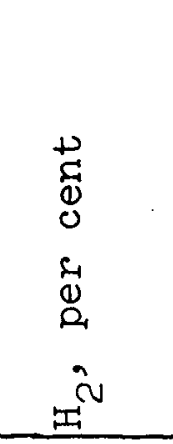 & 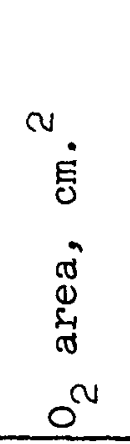 & 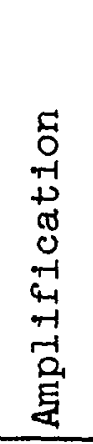 & $\begin{array}{l}\stackrel{+1}{c} \\
0 \\
0 \\
\varepsilon \\
0 \\
0 \\
0 \\
0^{\circ}\end{array}$ \\
\hline 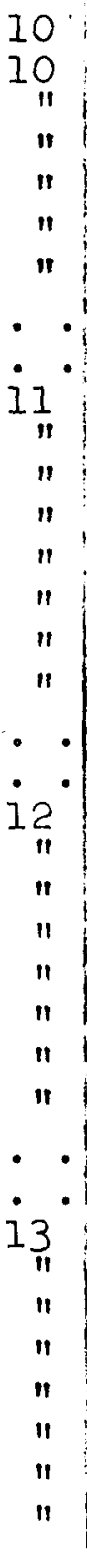 & $\begin{array}{c}4 \\
6 \\
7 \\
7 \\
8 \\
11 \\
\text { CaI. } \\
1 \\
9 \\
11 \\
10 \\
1 \\
11 \\
11 \\
12 \\
11 \\
\mathrm{CaI} \\
11 \\
1 \\
11 \\
2 \\
11 \\
3 \\
1 \\
4 \\
1 \\
\mathrm{CaI} . \\
1 \\
5 \\
11 \\
6 \\
7 \\
8 \\
11\end{array}$ & 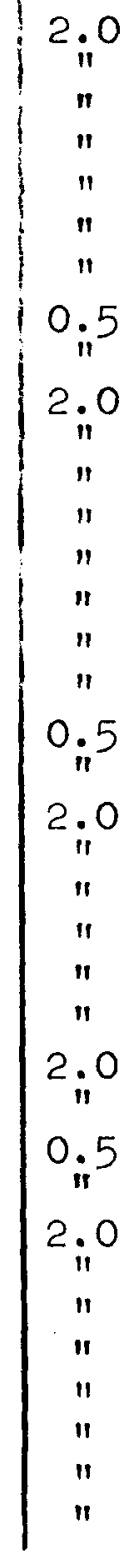 & 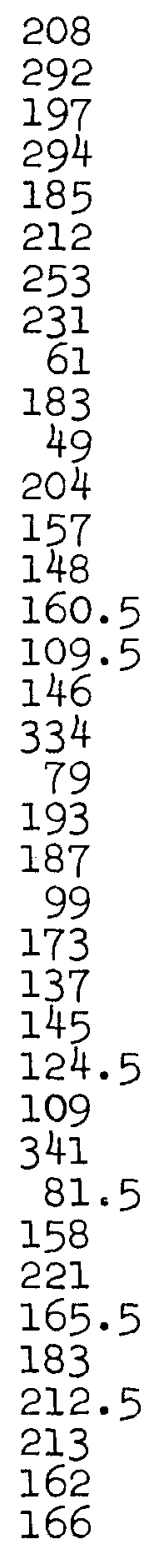 & $\begin{array}{l}26.6 \\
17.5 \\
32.9 \\
20.6 \\
25.9 \\
31.4 \\
37.8 \\
10.6 \\
20.9 \\
5.6 \\
38.9 \\
32.9 \\
25.0 \\
27.9 \\
20.9 \\
28.0 \\
54.9 \\
12.8 \\
54.6 \\
53.6 \\
54.6 \\
45.4 \\
12.9 \\
26.5 \\
23.7 \\
20.3 \\
21.6 \\
12.9 \\
28.7 \\
40.0 \\
27.3 \\
31.1 \\
35.8 \\
35.8 \\
5.4 \\
5.9\end{array}$ & $\begin{array}{l}\cdot \\
5 \\
5 \\
5 \\
5 \\
5 \\
5 \\
5 \\
5 \\
5 \\
5 \\
5 \\
5 \\
5 \\
5 \\
5 \\
5 \\
5 \\
5 \\
5 \\
5 \\
2 \\
1 \\
1 \\
2 \\
2 \\
2 \\
2 \\
5 \\
5 \\
5 \\
5 \\
5 \\
5 \\
5 \\
5 \\
5\end{array}$ & $\begin{array}{l}0.0770 \\
0.0752 \\
0.0945 \\
0.0942 \\
0.1035 \\
0.105 \\
0.532 \\
11 \\
0.0967 \\
0.0968 \\
0.161 \\
0.177 \\
0.143 \\
0.134 \\
0.162 \\
0.162 \\
0.532 \\
0.11 \\
0.239 \\
1.132 \\
1.11 \\
0.398 \\
0.3385 \\
0.403 \\
0.394 \\
0.532 \\
0.11 \\
0.154 \\
0.140 \\
0.144 \\
0.143 \\
0.142 \\
0.028 \\
0.030\end{array}$ & $\begin{array}{l}29.4 \\
45.8 \\
31.2 \\
44.8 \\
28.6 \\
32.1 \\
40.1 \\
. \cdot \\
.0 \\
73.2 \\
33.3 \\
25.3 \\
27.4 \\
25.4 \\
28.0 \\
22.7 \\
. \quad . \\
31.2 \\
27.4 \\
5.4 \\
4.4 \\
3.6 \\
6.5 \\
6.1 \\
5.1 \\
. \quad . \\
10.9 \\
14.0 \\
10.5 \\
13.0 \\
12.5 \\
12.7 \\
13.2 \\
13.1\end{array}$ & $\begin{array}{r}5 \\
5 \\
5 \\
5 \\
5 \\
5 \\
5 \\
\cdot . \\
\cdot \\
\cdot \\
5 \\
5 \\
5 \\
5 \\
5 \\
5 \\
5 \\
. \\
\cdot \\
5 \\
5 \\
2 \\
1 \\
1 \\
2 \\
2 \\
2 \\
. \\
. \\
5 \\
5 \\
5 \\
5 \\
5 \\
5 \\
5 \\
5\end{array}$ & 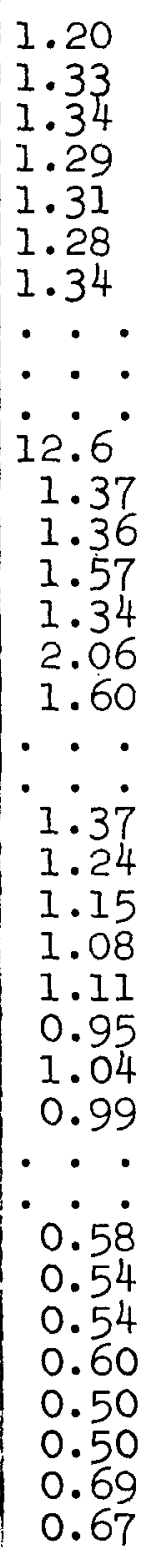 \\
\hline
\end{tabular}


142

TABLE 13 (contd.)

\begin{tabular}{|c|c|c|c|c|c|c|c|c|c|}
\hline 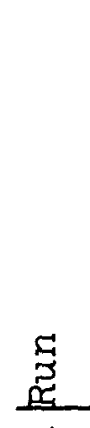 & $\begin{array}{r}0 \\
0 \\
-1 \\
0 \\
5 \\
5 \\
0 \\
\end{array}$ & $\begin{array}{l}\dot{0} \\
0 \\
0 \\
0 \\
5 \\
0_{1} \\
0 \\
\end{array}$ & 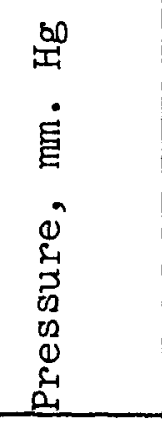 & 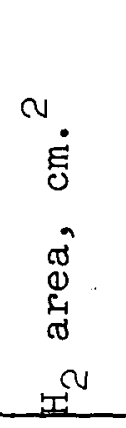 & 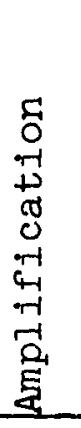 & 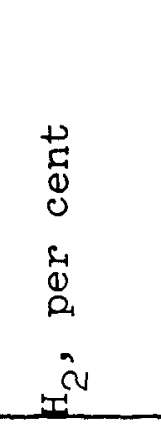 & 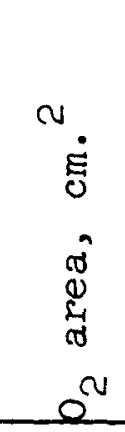 & 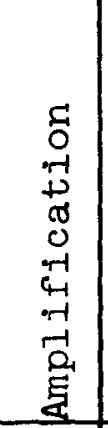 & 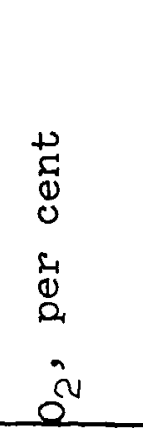 \\
\hline 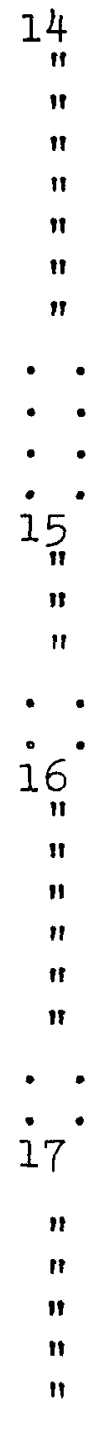 & $\begin{array}{c}9 \\
11 \\
10 \\
11 \\
11 \\
11 \\
12 \\
\text { Cal } \\
11 \\
11 \\
11 \\
1 \\
2 \\
3 \\
4 \\
\text { Ca1 } \\
11 \\
5 \\
6 \\
11 \\
7 \\
11 \\
8 \\
11 \\
\text { Cal } \\
11 \\
9 \\
10 \\
11 \\
11 \\
11 \\
12 \\
11\end{array}$ & 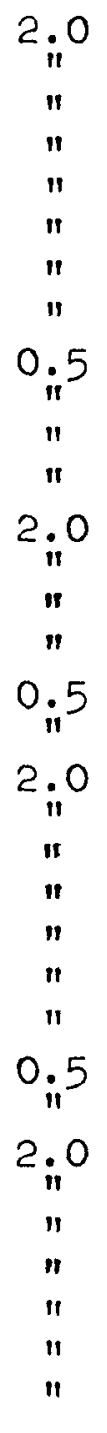 & $\begin{array}{l}247 \\
269 \\
140 \\
167 \\
183.5 \\
211 \\
169 \\
231.5 \\
354 \\
296 \\
109 \\
35 \\
375 \\
231 \\
195 \\
214 \\
334 \\
80 \\
227 \\
171 \\
143.5 \\
168 \\
158.5 \\
196 \\
169 \\
339 \\
69 \\
190 \\
225 \\
193 \\
184.5 \\
218 \\
318 \\
277\end{array}$ & $\begin{array}{l}0 . \\
0 \\
33.5 \\
37.5 \\
34.2 \\
31.9 \\
18.5 \\
18.5 \\
10.5 \\
32.1 \\
26.9 \\
60.0 \\
20.5 \\
12.4 \\
15.1 \\
33.1 \\
39.4 \\
35.7 \\
44.0 \\
38.0 \\
23.2 \\
25.3 \\
30.2 \\
25.4 \\
23.4 \\
29.0 \\
42.4 \\
37.0\end{array}$ & $\begin{array}{l}\cdot . \\
. \\
2 \\
2 \\
. \\
2 \\
. \\
2 \\
2 \\
2 \\
5 \\
10 \\
\cdot . \\
5 \\
5 \\
5 \\
2 \\
5 \\
. \\
2 \\
5 \\
5 \\
5 \\
5 \\
5 \\
2 \\
10 \\
0 . \\
2 \\
2 \\
2 \\
2 \\
2 \\
2\end{array}$ & 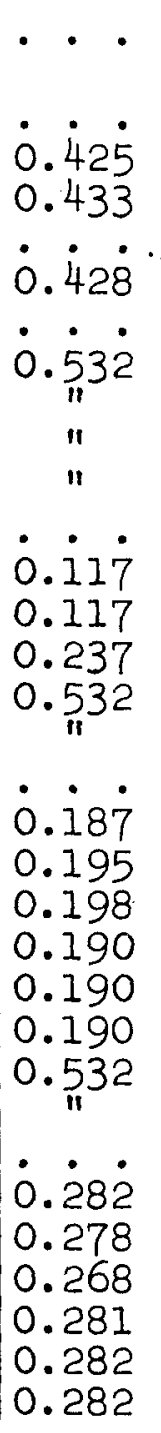 & 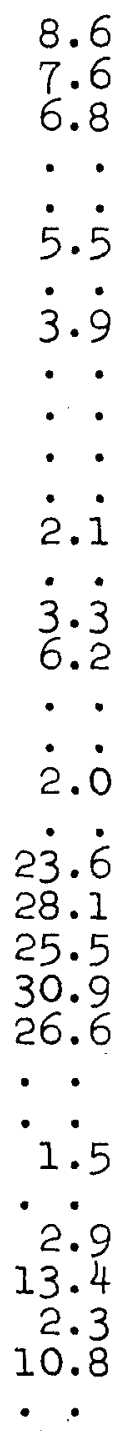 & 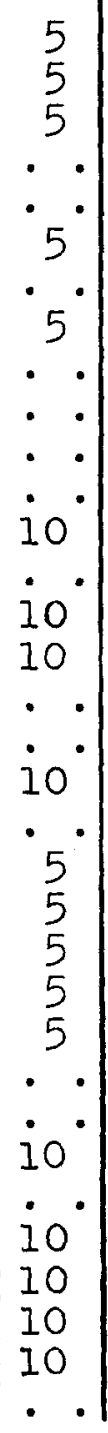 & 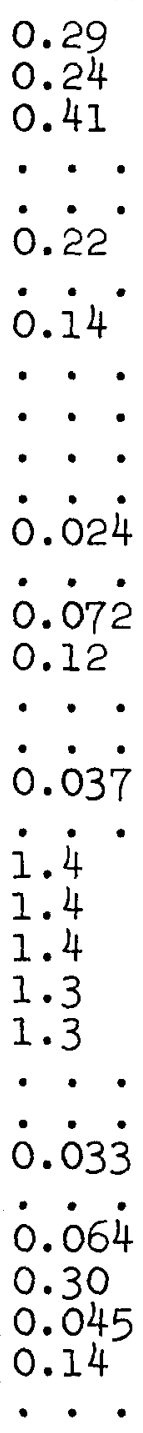 \\
\hline
\end{tabular}


143

TABLE 13 (contd.)

\begin{tabular}{|c|c|c|c|c|c|c|c|c|c|}
\hline$\underset{3}{5}$ & 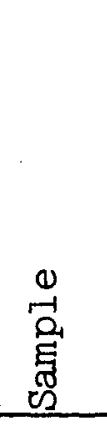 & $\begin{array}{l}0 \\
0 \\
0 \\
0 \\
5 \\
0 \\
0 \\
\end{array}$ & 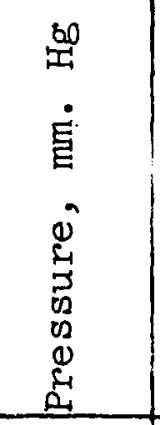 & 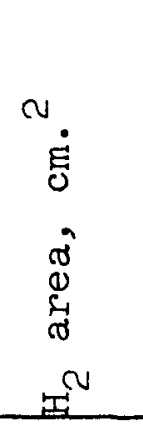 & 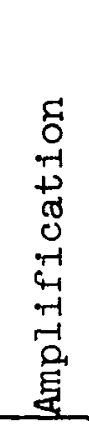 & 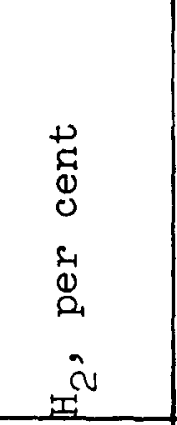 & 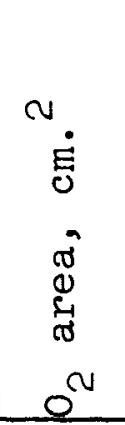 & 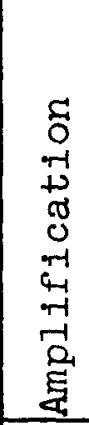 & 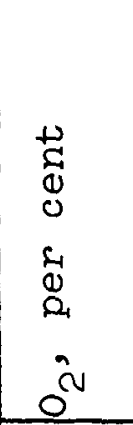 \\
\hline 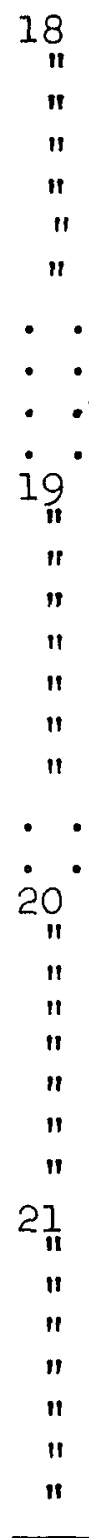 & $\begin{array}{c}5 \\
6 \\
11 \\
7 \\
1 \\
8 \\
1 " \\
\text { Cal. } \\
11 \\
1 " \\
11 \\
2 \\
2 \\
3 \\
11 \\
4 \\
11 \\
\text { Cal. } \\
11 \\
9 \\
11 \\
10 \\
11 \\
11 \\
12 \\
11 \\
1 \\
11 \\
2 \\
3 \\
4 \\
11 \\
5 \\
6 \\
\end{array}$ & 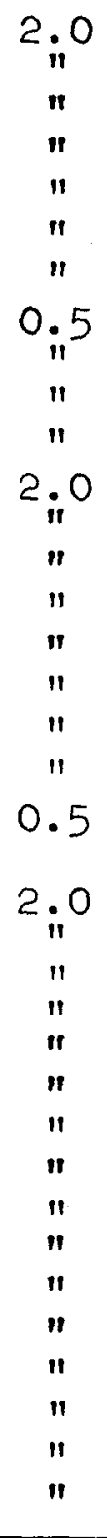 & $\begin{array}{l}210 \\
112 \\
296 \\
215 \\
281 \\
299 \\
203 \\
325 \\
86.5 \\
327 \\
97 \\
422 \\
371 \\
157 \\
160 \\
225 \\
234 \\
265 \\
201 \\
331 \\
71.5 \\
134 \\
104 \\
184 \\
198 \\
123 \\
92 \\
156.5 \\
121 \\
177 \cdot 5 \\
273.5 \\
226 \\
295 \\
267 \\
200 \\
229 \\
296\end{array}$ & $\begin{array}{l}9.3 \\
25.5 \\
17.1 \\
22.9 \\
25.8 \\
17.7 \\
19.5 \\
13.5 \\
20.5 \\
15.6 \\
\because .9 \\
21.0 \\
22.1 \\
25.7 \\
27.6 \\
25.5 \\
19.8 \\
19.7 \\
11.6 \\
43.7 \\
33.4 \\
0.0 \\
38.6 \\
30.2 \\
26.4 \\
40.7 \\
3.4 \\
5.0 \\
3.9 \\
5.0 \\
5.3 \\
7.1 \\
8.9 \\
1.4\end{array}$ & $\begin{array}{r}\cdot \\
2 \\
2 \\
2 \\
2 \\
2 \\
2 \\
2 \\
5 \\
2 \\
5 \\
. \therefore \\
2 \\
2 \\
2 \\
2 \\
2 \\
2 \\
2 \\
2 \\
5 \\
2 \\
2 \\
0 . \\
2 \\
2 \\
1 \\
2 \\
5 \\
5 \\
5 \\
5 \\
5 \\
10 \\
10 \\
10\end{array}$ & $\begin{array}{l}0.176 \\
0.182 \\
0.168 \\
0.173 \\
0.183 \\
0.184 \\
0.532 \\
11 \\
11 \\
0 . . \\
0.287 \\
0.292 \\
0.242 \\
0.250 \\
0.204 \\
0.208 \\
0.532 \\
0.690 \\
0.680 \\
0.00 \\
0.664 \\
0.695 \\
0.714 \\
0.710 \\
0.0162 \\
0.0155 \\
0.0146 \\
0.0143 \\
0.0168 \\
0.0150 \\
0.0165 \\
0.0020\end{array}$ & $\begin{array}{l}14.4 \\
7.8 \\
17.6 \\
12.2 \\
17.0 \\
16.0 \\
11.4 \\
: \quad \\
: \vdots \\
12.9 \\
88.7 \\
16.9 \\
14.7 \\
14.9 \\
44.9 \\
25.0 \\
0 . \\
.0 \\
8.8 \\
6.6 \\
24.2 \\
27.5 \\
11.1 \\
6.4 \\
5.0 \\
7.8 \\
13.5 \\
20.2 \\
18.8 \\
21.8 \\
22.9 \\
29.6 \\
41.9 \\
28.1\end{array}$ & 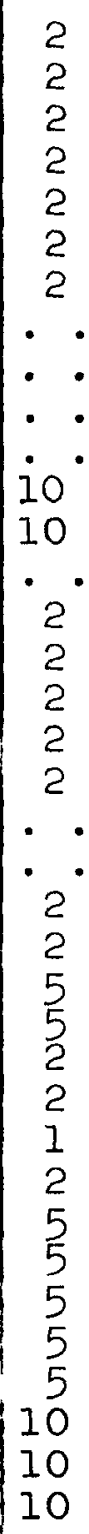 & 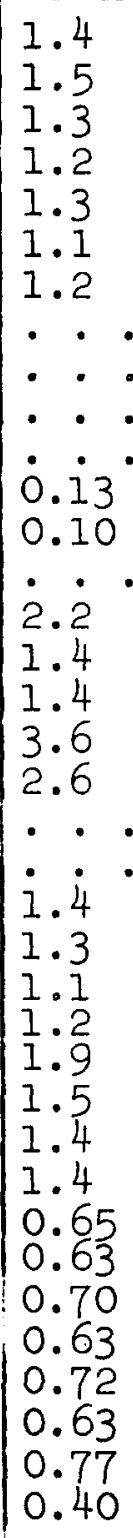 \\
\hline
\end{tabular}




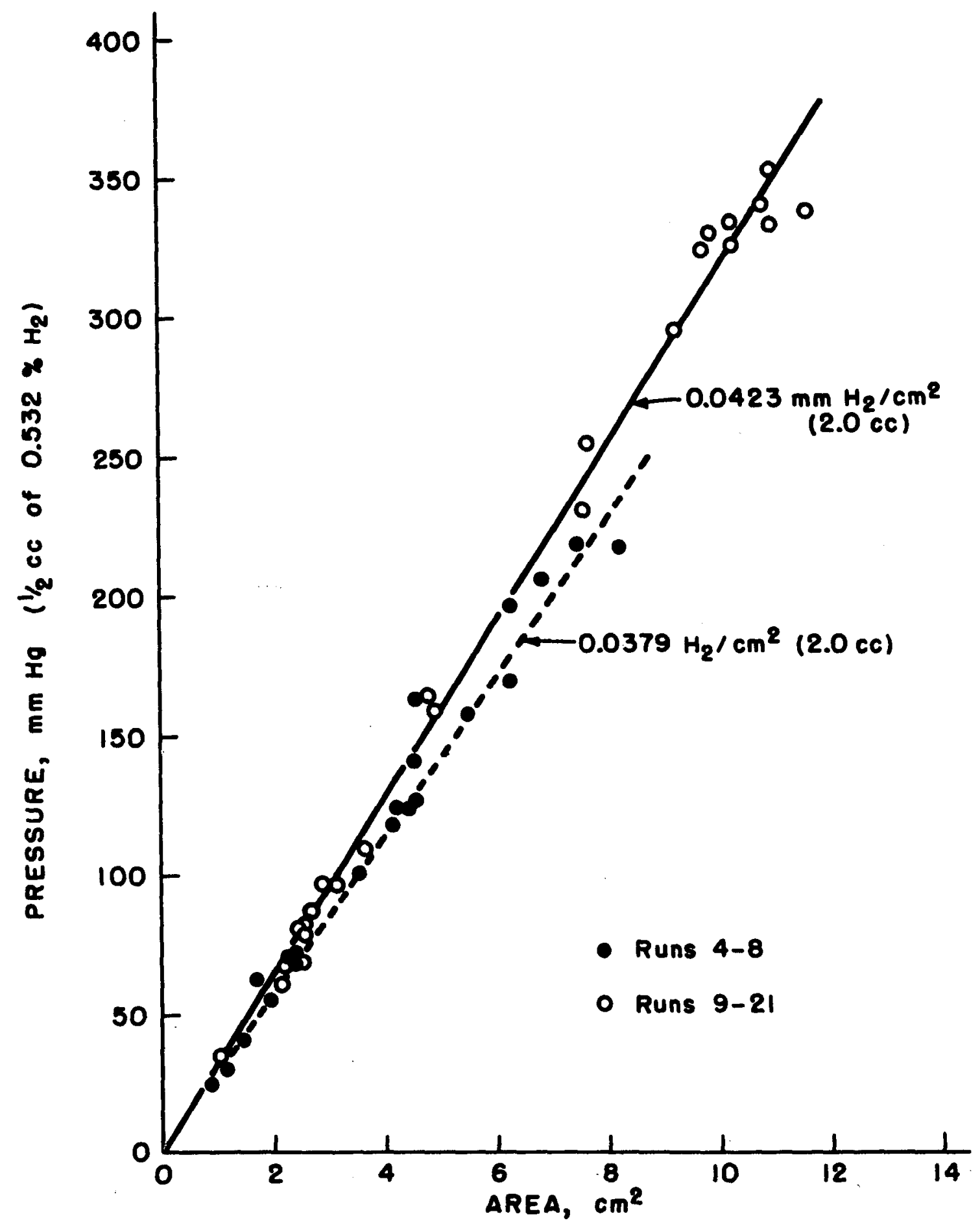

Figure 27. Hydrogen analysis colibration. 
TABLE 14

FISSION PRODUCT ANALYSES

\begin{tabular}{|c|c|c|c|c|c|c|c|c|c|}
\hline \multirow[t]{2}{*}{ Run } & \multicolumn{5}{|c|}{ Fissions per Gram in the Medium } & \multicolumn{3}{|c|}{ Fissions per Run } & \multirow{2}{*}{$\begin{array}{l}\text { Fraction } \\
\text { in } \\
\text { Liquid }\end{array}$} \\
\hline & Liquid, Mo99 & Solid, Mo 99 & Liquid, & $\mathrm{Ce}$ & 142 & Liquid & Solid & Total & \\
\hline 5 & $5.26 \times 10^{10}$ & $\cdot \cdot \cdot \cdot \cdot \cdot$ & $\cdot \cdot \cdot \cdot$ & - & - & $\cdot \cdot \cdot \cdot \cdot$ & $\cdot \cdot \cdot \cdot \cdot$ & $50.5 \times 10^{10}$ & . $\cdot \cdot \cdot$ \\
\hline 6 & 24.8 & . . . . . . & . . . & - & - & $\cdot \cdot \cdot \cdot$ & $\cdot \cdot \cdot \cdot$ & 237 & . $\cdot \cdot$ \\
\hline 8 & 7.08 & . . . . . & • • • • & - & - & $\ldots \ldots$ & ... • . & $67 \cdot 9$ & . $\cdot \cdot$ \\
\hline 9 & 103.8 & $286 \times 10^{10}$ & . . . & $\bullet$ & - & $935 \times 10^{10}$ & $1,430 \times 10^{10}$ & 2,365 & 0.40 \\
\hline 10 & 84.9 & 265 & . . . . & - & - & 760 & 1,300 & 2,060 & 0.37 \\
\hline 11 & 67.4 & $526 \pm 22 \%$ & $\cdot \cdot \cdot \cdot$ & - & - & 566 & 5,420 & 5,988 & 0.10 \\
\hline 12 & 162.4 & $298 \pm 9 \%$ & |. . . & $\because$ & - & 1,200 & 5,820 & 7,020 & 0.17 \\
\hline 13 & 81.3 & $487 \pm 24 \%$ & $0.768 \times 10$ & $0^{10}$ & & 722 & 2,490 & 3.212 & 0.23 \\
\hline 14 & 256.5 & 256 & . . . . & - & - & 2,100 & 2,710 & 4,810 & 0.44 \\
\hline 15 & 58.7 & 227 & 0.448 & & & 457 & 2,310 & 2,767 & 0.17 \\
\hline 16 & 56.9 & $90 \pm 46 \not 6$ & 0.357 & & & 496 & 243 & 739 & 0.67 \\
\hline 17 & 62.7 & 54 & |. . • & . & - & 532 & 209 & 741 & 0.72 \\
\hline 18 & 95.7 & 180 & |. . . & - & - & 775 & 1,920 & 2,695 & 0.29 \\
\hline 19 & 69.1 & 128 & . . . . & - & - & 581 & 644 & 1,225 & 0.47 \\
\hline 20 & 185.0 & 87 & 2.10 & & & 1,465 & 875 & 2,340 & 0.63 \\
\hline
\end{tabular}


fissions occurring during the mun was calculated. The last column in the table shows the fraction of the moIybdenum-99 found in the Iiquid phase.

After the fission products had been analyzed, the liquid phase was analyzed for its uranium content. The results are given as the fraction of the original amount of uranium which ended up in solution: for the 6 micron size, $0.73 \times 10^{-4}$; for the $8 \mathrm{mlcron}, 0.48 \times 10^{-4}$; for the 16 micron, $0.12 \times 10^{-4}$, and for the $50 \mathrm{micron}, 0.46 \times 10^{-4}$. Except for the large size, the results appear to be proportional to the surface area per unit volume. It is not known if the dissolution of uranium was a result of the irradiation; Sowden et al. (36) reported such a dissolution effect in the irradiation of plutonium slurries. For the purpose of this work the amount of uranium in solution was so small that it was neglected in the measurement of the average particle size. Neutron flux measurements

In Table 15 are shown the results of the analyses of the chromium nitrate flux monitors. The welght of the crystal irradiated is given in the first column. The number of counts recorded under the peak of the gamma energy spectrum, shown in Figure 28, in one minute is given in the second column. The half-life of 27.8 days and the time lapse between the time that the irradiation was stopped and that when the count was taken were used to calculate the decay 
TABLE 15

NEUTRON FLUX MONITOR

\begin{tabular}{|c|c|c|c|c|c|}
\hline Run & $\begin{array}{l}\text { Weight, mg. } \\
\operatorname{Cr}\left(\mathrm{NO}_{3}\right)_{3} \cdot 9 \mathrm{H}_{2} \mathrm{O}\end{array}$ & $\begin{array}{l}\text { Counts } \\
(1 \mathrm{~min} .)\end{array}$ & $\begin{array}{c}\text { Irradiation Time, } \\
\text { min. }\end{array}$ & Decay Factor & $\begin{array}{l}\text { Relative Flux } \\
\text { Counts/mg/min }\end{array}$ \\
\hline 9 & 72.4 & 75,542 & 32.25 & 0.704 & 45.9 \\
\hline 10 & 47.6 & 52,229 & 32.6 & 0.714 & 47.1 \\
\hline 11 & 39.9 & 42,529 & 31.0 & 0.728 & 47.1 \\
\hline 12 & 51.7 & 57.677 & $31 \cdot 5$ & 0.740 & $47 \cdot 7$ \\
\hline 13 & 57.4 & 50.686 & 25.0 & 0.745 & 47.4 \\
\hline 14 & $59 \cdot 5$ & 51,699 & 24.5 & 0.751 & 47.1 \\
\hline 15 & 60.7 & 56.181 & $27 \cdot 2$ & 0.763 & $44.7^{a}$ \\
\hline 16 & 56.8 & 56.205 & 25.25 & 0.774 & 50.6 \\
\hline 17 & $42 \cdot 5$ & 38,793 & $24 \cdot 3$ & 0.782 & $47 \cdot 9$ \\
\hline 18 & $50 \cdot 5$ & 48.751 & 25.25 & 0.809 & 45.4 \\
\hline 19 & 56.8 & 61,077 & 30.25 & 0.788 & 45.1 \\
\hline 20 & 48.0 & 40,412 & 25.1 & 0.818 & $41.0^{a}$ \\
\hline 21 & $\{59.4$ & $\{74,344$. & 33.75 & 0.829 & $\{44.8$ \\
\hline & 58.2 & 73,317 & & & 45.0 \\
\hline
\end{tabular}

${ }^{a_{S}}$ Some sample lost.

Mean flux is $46.8 \pm 1.4$. 


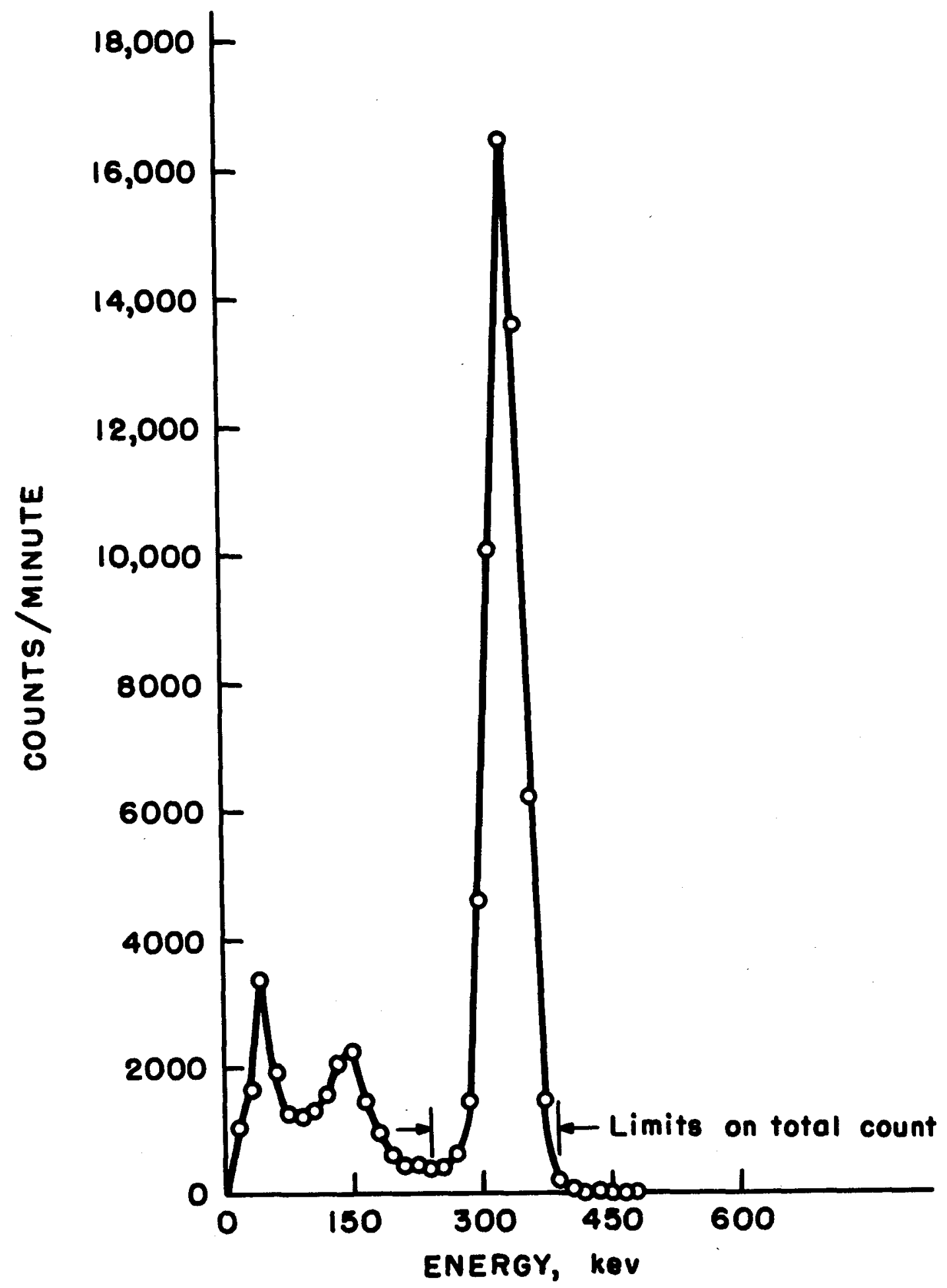

Figure 28. Gammo energy spectrum from chromium nitrate. (Run 15) 
factor. The last column, a measure of the relative neutron flux during a run, was obtained by dividing the total number of counts, corrected for decay, by the weight of chromium nitrate and the length of irradiation.

Note that no data are given for the runs 4 through 8; some of the sample from these runs was lost in an attempt to remove the chromium nitrate from the aluminum foll. It was for this reason that the rest of the samples were counted with the chromium inside the foil. Even so, it was observed that some of the molten nitrate (m.p. $=37^{\circ} \mathrm{C}$ ) had escaped during the irradiation for the runs noted in the table.

Particle size analyses

In Table 16 are given the data used to determine the particle size as a function of the sedimentation rate. Since the amount of sample left from runs 16 and 17 was not enough for this analysis, these samples were combined with that from runs 19 and 20, respectively. Density and viscosity of the glycerol-water mixture at $25^{\circ} \mathrm{C}$. are given in the table. By combining this data with the density of the solid, $9.75 \mathrm{~g} / \mathrm{cc}$, and the height of liquid above the counting zone, $10 \mathrm{~cm}$., to the relationship between particle size and settling time given in the last column was determined.

Table 17 gives the data obtained during the sedimentation, that is, the total counts versus settling time. 
TABLE 16

PARTICLE SIZE ANALYSES-PARAMETERS

\begin{tabular}{|c|c|c|c|c|c|}
\hline \multicolumn{3}{|c|}{ Solid Phase } & \multicolumn{2}{|c|}{ Liquid Phase } & \multirow{2}{*}{$\begin{array}{c}D(t)^{1 / 2} \\
\mu(\min .)^{1 / 2}\end{array}$} \\
\hline$\overline{\text { Size }}$ & Run & Weight, $\mathrm{gm}$. & Dens1ty, $\mathrm{gm} / \mathrm{cc}$ & Viscosity, cp & \\
\hline Large & 11 & $3 \cdot 5$ & 1.881 & 21.69 & 88.0 \\
\hline Large & 18 & $4: 5$ & 1.881 & 21.69 & 88.0 \\
\hline Medium & 13 & $3 \cdot 5$ & 1.1228 & 4.648 & 40.6 \\
\hline Medium & 14 & 4.5 & 1.1228 & 4.648 & 40.6 \\
\hline Small & $\left\{\begin{array}{l}16 \\
19\end{array}\right.$ & $\left\{\begin{array}{l}1.0 \\
3.0\end{array}\right.$ & 1.00 & 0.893 & 17.65 \\
\hline Fine & $\left\{\begin{array}{l}17 \\
20\end{array}\right.$ & $\left\{\begin{array}{l}1 \cdot 0 \\
3 \cdot 5\end{array}\right.$ & 1.00 & 0.893 & 17.65 \\
\hline
\end{tabular}


TABLE 17

PARTICLE SIZE ANALYSES - SEDIMENTATION RATE

RUN IIA

\begin{tabular}{|c|c|c|c|c|c|}
\hline \multirow[b]{2}{*}{$\begin{array}{l}\text { Time, } \\
\text { min. }\end{array}$} & \multirow[b]{2}{*}{ Counts } & \multirow[b]{2}{*}{$\begin{array}{l}\text { Count Rate, } \\
\text { Counts/min }\end{array}$} & \multicolumn{2}{|c|}{ Corrected for Background } & \multirow[b]{2}{*}{$\mathrm{D}, \mu$} \\
\hline & & & $\begin{array}{l}\text { Count Rate, } \\
\text { Counts/min }\end{array}$ & $\begin{array}{l}\text { Fraction of } \\
\text { Initial Rate, } \\
\text { per cent }\end{array}$ & \\
\hline $\begin{array}{l}0.2 \\
0.4 \\
0.6 \\
0.8 \\
1.0 \\
1.2 \\
1.4 \\
1.6 \\
1.8 \\
2.0 \\
2.2 \\
2.4 \\
2.6 \\
2.8 \\
3.0 \\
3.2 \\
3.4 \\
3.6 \\
3.8 \\
4.0 \\
5.0 \\
6.0 \\
7.0 \\
8.0 \\
9.0 \\
10 \\
15 \\
20 \\
25 \\
30 \\
60\end{array}$ & $\begin{array}{r}1,100 \\
2,250 \\
3,360 \\
4,350 \\
5,300 \\
6,430 \\
7,550 \\
8,600 \\
9,750 \\
10,620 \\
11,550 \\
12,450 \\
13,030 \\
13,650 \\
14,140 \\
14,520 \\
14,780 \\
15,030 \\
15,260 \\
15,480 \\
16,270 \\
17,050 \\
17,730 \\
18,470 \\
19,150 \\
19,820 \\
23,140 \\
26,400 \\
29,670 \\
32,780 \\
52,610\end{array}$ & $\begin{array}{r}5,600 \\
4,950 \\
4,750 \\
5,650 \\
5,600 \\
5,250 \\
4,750 \\
5,250 \\
4,650 \\
4,500 \\
2,900 \\
3,100 \\
2,450 \\
1,900 \\
1,300 \\
1,250 \\
1,150 \\
1,100 \\
790 \\
820 \\
680 \\
740 \\
680 \\
670 \\
660 \\
650 \\
650 \\
620 \\
660\end{array}$ & $\begin{array}{r}4,950 \\
4,300 \\
4,100 \\
5,000 \\
4,950 \\
4,600 \\
4,100 \\
4,600 \\
4,000 \\
3,850 \\
2,250 \\
2,450 \\
1,800 \\
1,250 \\
650 \\
600 \\
500 \\
450 \\
140 \\
170 \\
30 \\
90 \\
30 \\
20 \\
10 \\
0 \\
0 \\
0 \\
0\end{array}$ & $\begin{array}{r}100 \\
87 \\
83 \\
100 \\
100 \\
93 \\
83 \\
93 \\
81 \\
78 \\
45 \\
50 \\
36 \\
25 \\
13 \\
12 \\
10 \\
9 \\
3 \\
3 \\
0.6 \\
2 \\
0.6 \\
0.4 \\
0.2 \\
0 \\
0 \\
0 \\
0 \\
0\end{array}$ & $\begin{array}{l}114 \\
98 \\
88 \\
80 \\
74 \\
70 \\
65 \cdot 5 \\
62 \\
59 \cdot 5 \\
57 \\
54 \cdot 5 \\
52 \cdot 5 \\
51 \\
49 \\
47 \cdot 5 \\
46 \cdot 5 \\
45 \\
44 \\
39 \\
36 \\
33 \\
31 \\
29 \\
28 \\
23 \\
19 \\
17 \\
16 \\
11\end{array}$ \\
\hline
\end{tabular}

contd. 
TABLE 17 (contd.)

RUN IIB

\begin{tabular}{|c|c|c|c|c|c|}
\hline \multirow[b]{2}{*}{$\begin{array}{l}\text { Time, } \\
\text { min. }\end{array}$} & \multirow[b]{2}{*}{ Counts } & \multirow[b]{2}{*}{$\begin{array}{l}\text { Count Rate, } \\
\text { Counts/min }\end{array}$} & \multicolumn{2}{|c|}{ Corrected for Background } & \multirow[b]{2}{*}{$D, \mu$} \\
\hline & & & $\begin{array}{l}\text { Count Rate, } \\
\text { Counts } / \text { min }\end{array}$ & $\begin{array}{l}\text { Fraction of } \\
\text { Initial Rate, } \\
\text { per cent }\end{array}$ & \\
\hline 0.2 & 1,080 & & & & \\
\hline 0.6 & 3,190 & 5,320 & 4,690 & 100 & 114 \\
\hline 0.8 & 4,280 & 5,450 & 4,820 & 102 & 98 \\
\hline 1.0 & 5,310 & 5,150 & $\begin{array}{l}4,520 \\
4,720\end{array}$ & $\begin{array}{r}97 \\
700\end{array}$ & 88 \\
\hline $\begin{array}{l}1.2 \\
1.4\end{array}$ & $\begin{array}{l}0,380 \\
7,350\end{array}$ & $\begin{array}{l}5,350 \\
4,850\end{array}$ & $\begin{array}{l}4,720 \\
4,220\end{array}$ & $\begin{array}{r}100 \\
90\end{array}$ & 74 \\
\hline $\begin{array}{l}1.6 \\
1.8\end{array}$ & $\begin{array}{l}8,450 \\
9,320\end{array}$ & $\begin{array}{l}5,250 \\
4,600\end{array}$ & $\begin{array}{l}4,620 \\
3,970\end{array}$ & $\begin{array}{l}99 \\
85\end{array}$ & $\begin{array}{l}70 \\
65.5\end{array}$ \\
\hline 2.0 & 10,190 & 4,350 & 3,720 & 79 & 62 \\
\hline $\begin{array}{l}2.2 \\
2.4\end{array}$ & 11,060 & $\begin{array}{l}4,350 \\
3,750\end{array}$ & $\begin{array}{l}3,720 \\
3,120\end{array}$ & $\begin{array}{l}79 \\
66\end{array}$ & $\begin{array}{l}59 \cdot 5 \\
57\end{array}$ \\
\hline 2.6 & 12,420 & 3,050 & 2,420 & 51 & 54.5 \\
\hline $\begin{array}{l}2.8 \\
3.0\end{array}$ & $\begin{array}{l}12,950 \\
13,380\end{array}$ & $\begin{array}{l}2,650 \\
2,750\end{array}$ & $\begin{array}{l}2,020 \\
1,520\end{array}$ & $\begin{array}{l}43 \\
32\end{array}$ & 52.5 \\
\hline 3.2 & 13,670 & 1,450 & 820 & 17.5 & 49 \\
\hline 3.4 & 13,900 & 1,150 & $\begin{array}{l}520 \\
420\end{array}$ & 11 & $\begin{array}{l}47.5 \\
46.5\end{array}$ \\
\hline $\begin{array}{l}3.0 \\
3.8\end{array}$ & $\begin{array}{l}14,110 \\
14,300\end{array}$ & $\begin{array}{r}1,050 \\
950\end{array}$ & 320 & 9 & $\begin{array}{l}40 . \\
45\end{array}$ \\
\hline 4.0 & 14,480 & 900 & 270 & 6 & 44 \\
\hline & 15 & 830 & 200 & 4 & 39 \\
\hline 6.0 & 15,950 & 700 & 70 & 1.5 & 36 \\
\hline 7.0 & 16,640 & 690 & 60 & $1 \cdot 3$ & 33 \\
\hline 8.0 & 17,290 & 65 & 20 & 0. & 31. \\
\hline$y$ & 17,960 & 670 & 40 & 0.8 & 29 \\
\hline 10 & 18,640 & 680 & 50 & 1.0 & 28 \\
\hline 23 & 26,880 & 630 & 0 & 0 & 18 \\
\hline & 4 & 630 & 0 & 0 & 12 \\
\hline
\end{tabular}


TABLE 17 (contd.)

RUN 18A

\begin{tabular}{|c|c|c|c|c|c|}
\hline \multirow[b]{2}{*}{$\begin{array}{l}\text { Time, } \\
\text { min. }\end{array}$} & \multirow[b]{2}{*}{ Counts } & \multirow[b]{2}{*}{$\begin{array}{l}\text { Count Rate, } \\
\text { Counts/min }\end{array}$} & \multicolumn{2}{|c|}{ Corrected for Background } & \multirow[b]{2}{*}{$D, \mu$} \\
\hline & & & $\begin{array}{l}\text { Count Rate, } \\
\text { Counts } / \text { min }\end{array}$ & $\begin{array}{l}\text { Fraction of } \\
\text { Inftial Rate, } \\
\text { per cent }\end{array}$ & \\
\hline $\begin{array}{l}0.2 \\
0.4 \\
0.6 \\
0.8 \\
1.0 \\
1.2 \\
1.4 \\
1.6 \\
1.8 \\
2.0 \\
2.2 \\
2.4 \\
2.6 \\
2.8 \\
3.0 \\
3.2 \\
3.4 \\
3.6 \\
3.8 \\
4.0 \\
6.0 \\
10 \\
15 \\
20 \\
27\end{array}$ & $\begin{array}{r}1,500 \\
3,000 \\
4,500 \\
6,000 \\
7,600 \\
9,000 \\
10,400 \\
11,900 \\
13,200 \\
14,700 \\
16,100 \\
17,300 \\
18,600 \\
19,800 \\
20,900 \\
21,910 \\
22,800 \\
23,500 \\
24,100 \\
24,520 \\
26,710 \\
29,610 \\
33,190 \\
36,560 \\
41,390\end{array}$ & $\begin{array}{r}7,500 \\
7,500 \\
8,000 \\
7,000 \\
7,000 \\
7,500 \\
6,500 \\
7,500 \\
7,000 \\
6,000 \\
6,500 \\
6,000 \\
5,500 \\
5,050 \\
4,450 \\
3,500 \\
3,000 \\
2,100 \\
1,100 \\
725 \\
716 \\
674 \\
690\end{array}$ & $\begin{array}{r}6,800 \\
6,800 \\
7,300 \\
6,300 \\
6,300 \\
6,800 \\
5,800 \\
6,800 \\
6,300 \\
5,300 \\
5,800 \\
5,300 \\
4,800 \\
4,350 \\
3,750 \\
2,800 \\
2,300 \\
1,400 \\
410 \\
35 \\
26 \\
0 \\
0\end{array}$ & $\begin{array}{c}100 \\
100 \\
107 \\
93 \\
93 \\
100 \\
85 \\
100 \\
93 \\
78 \\
85 \\
78 \\
71 \\
64 \\
55 \\
41 \\
34 \\
20.5 \\
6 \\
0.5 \\
0.4 \\
0 \\
0\end{array}$ & $\begin{array}{l}114 \\
98 \\
88 \\
80 \\
74 \\
70 \\
65.5 \\
62 \\
59.5 \\
57 \\
54.5 \\
52.5 \\
51 \\
49 \\
47.5 \\
46.5 \\
45 \\
44 \\
36 \\
28 \\
23 \\
19 \\
17\end{array}$ \\
\hline
\end{tabular}


TABLE 17 (contd.)

RUN 18B

\begin{tabular}{|c|c|c|c|c|c|}
\hline \multirow[b]{2}{*}{$\begin{array}{l}\text { Time, } \\
\text { min. }\end{array}$} & \multirow[b]{2}{*}{ Counts } & \multirow[b]{2}{*}{$\begin{array}{l}\text { Count Rate, } \\
\text { Counts/min }\end{array}$} & \multicolumn{2}{|c|}{ Corrected for Background } & \multirow[b]{2}{*}{$D, \mu$} \\
\hline & & & $\begin{array}{l}\text { Count Rate, } \\
\text { Counts/min }\end{array}$ & $\begin{array}{l}\text { Fraction of } \\
\text { Initial Rate, } \\
\text { per cent }\end{array}$ & \\
\hline $\begin{array}{l}0.2 \\
0.4 \\
0.6 \\
0.8 \\
1.0 \\
1.2 \\
1.4 \\
1.6 \\
1.8 \\
2.0 \\
2.2 \\
2.4 \\
2.6 \\
2.8 \\
3.0 \\
3.2 \\
3.4 \\
3.6 \\
3.8 \\
4.0 \\
5.0 \\
6.0 \\
7.0 \\
8.0 \\
9.0 \\
10 \\
5\end{array}$ & $\begin{array}{r}1,500 \\
3,050 \\
4,550 \\
6,050 \\
7,550 \\
9,060 \\
10,560 \\
12,100 \\
13,530 \\
14,930 \\
16,360 \\
17,650 \\
19,030 \\
20,340 \\
21,440 \\
22,540 \\
23,480 \\
24,310 \\
24,950 \\
25,400 \\
26,790 \\
27,660 \\
28,460 \\
29,210 \\
29,940 \\
30,640 \\
34,310 \\
37,850 \\
44,830\end{array}$ & $\begin{array}{r}7,580 \\
7,500 \\
7,500 \\
7,550 \\
7,500 \\
7,700 \\
7,150 \\
7,000 \\
7,150 \\
6,450 \\
6,900 \\
6,550 \\
5,500 \\
5,500 \\
4,700 \\
4,150 \\
3,200 \\
2,250 \\
1,390 \\
870 \\
800 \\
750 \\
730 \\
700 \\
734 \\
708 \\
698\end{array}$ & $\begin{array}{r}6,880 \\
6,800 \\
6,800 \\
6,850 \\
6,800 \\
7,000 \\
6,450 \\
6,300 \\
6,450 \\
5,750 \\
6,200 \\
5,850 \\
4,800 \\
4,800 \\
4,000 \\
3,450 \\
2,500 \\
1,550 \\
690 \\
170 \\
100 \\
50 \\
30 \\
0 \\
34 \\
0 \\
0\end{array}$ & $\begin{array}{r}100 \\
99 \\
99 \\
100 \\
99 \\
102 \\
94 \\
92 \\
92 \\
94 \\
84 \\
90 \\
85 \\
70 \\
70 \\
58 \\
50 \\
36 \\
22.5 \\
10 \\
2.5 \\
1.5 \\
0.7 \\
0.4 \\
0 \\
0.4 \\
0 \\
0\end{array}$ & $\begin{array}{l}114 \\
98 \\
88 \\
80 \\
74 \\
70 \\
65.5 \\
62 \\
59 \cdot 5 \\
57 \\
54 \cdot 5 \\
52.5 \\
51 \\
49 \\
47 \cdot 5 \\
46 \cdot 5 \\
45 \\
44 \\
39 \\
36 \\
33 \\
31 \\
29 \\
28 \\
23 \\
19 \\
16\end{array}$ \\
\hline
\end{tabular}


TABLE 17 (contd.)

RUN 13A

\begin{tabular}{|c|c|c|c|c|c|}
\hline \multirow[b]{2}{*}{$\begin{array}{l}\text { Time, } \\
\text { min. }\end{array}$} & \multirow[b]{2}{*}{ Counts } & \multirow[b]{2}{*}{$\begin{array}{l}\text { Count Rate, } \\
\text { Counts/min }\end{array}$} & \multicolumn{2}{|c|}{ Corrected for Background } & \multirow[b]{2}{*}{$D, \mu$} \\
\hline & & & $\begin{array}{l}\text { Count Rate, } \\
\text { Counts/min }\end{array}$ & $\begin{array}{l}\text { Fraction of } \\
\text { Inttial Rate, } \\
\text { per cent }\end{array}$ & \\
\hline $\begin{array}{l}0.5 \\
1.0 \\
1.5 \\
2.0 \\
2.5 \\
3.0 \\
3.5 \\
4.0 \\
4.5 \\
5.0 \\
5.5 \\
6.0 \\
6.5 \\
7.0 \\
7.5 \\
8.0 \\
8.5 \\
9.0 \\
9.5 \\
10.0 \\
10.5 \\
11.0 \\
11.5 \\
12.0 \\
13 \\
14 \\
15 \\
17 \\
20 \\
26 \\
46 \\
55\end{array}$ & $\begin{array}{r}2,400 \\
4,880 \\
7,270 \\
9,650 \\
12,000 \\
14,250 \\
16,280 \\
18,330 \\
20,140 \\
21,720 \\
23,200 \\
24,390 \\
25,300 \\
26,130 \\
26,810 \\
27,380 \\
27,910 \\
28,350 \\
28,780 \\
29,190 \\
29,610 \\
30,020 \\
30,390 \\
30,780 \\
31,450 \\
32,170 \\
32,890 \\
34,190 \\
36,200 \\
40,240 \\
52,810 \\
58,440\end{array}$ & $\begin{array}{r}4,170 \\
4,400 \\
4,160 \\
4,100 \\
3,620 \\
3,160 \\
2,960 \\
2,380 \\
1,820 \\
1,660 \\
1,360 \\
1,140 \\
1,060 \\
880 \\
860 \\
820 \\
840 \\
820 \\
740 \\
780 \\
670 \\
720 \\
720 \\
650 \\
660 \\
670 \\
630 \\
630\end{array}$ & $\begin{array}{r}4,070 \\
3,770 \\
3,530 \\
3,470 \\
2,990 \\
2,530 \\
2,330 \\
1,750 \\
1,190 \\
1.030 \\
730 \\
510 \\
430 \\
250 \\
230 \\
190 \\
210 \\
190 \\
110 \\
150 \\
40 \\
90 \\
90 \\
20 \\
30 \\
40 \\
0 \\
0\end{array}$ & $\begin{array}{l}100 \\
90 \\
85 \\
83 \\
72 \\
61 \\
56 \\
42 \\
29 \\
25 \\
17.5 \\
12 \\
10 \\
6 \\
5.5 \\
4.5 \\
5 \\
4.5 \\
2.5 \\
3.5 \\
1 \\
2 \\
2 \\
0.5 \\
0.5 \\
1 \\
0 \\
0\end{array}$ & $\begin{array}{l}25.7 \\
23.4 \\
21.7 \\
20.3 \\
19.2 \\
18.2 \\
17.3 \\
16.6 \\
15.9 \\
15.3 \\
14.8 \\
14.4 \\
13.9 \\
13.5 \\
13.2 \\
12.8 \\
12.5 \\
12.2 \\
12.0 \\
11.7 \\
11.3 \\
10.8 \\
10.5 \\
9.8 \\
9.1 \\
8.0 \\
6.0 \\
5.5\end{array}$ \\
\hline
\end{tabular}


TABLE 17 (contd.)

RUN 14A

\begin{tabular}{|c|c|c|c|c|c|}
\hline \multirow[b]{2}{*}{$\begin{array}{l}\text { Time, } \\
\text { min. }\end{array}$} & \multirow[b]{2}{*}{ Counts } & \multirow[b]{2}{*}{$\begin{array}{l}\text { Count Rate, } \\
\text { Counts } / \mathrm{min}\end{array}$} & \multicolumn{2}{|c|}{ Corrected for Background } & \multirow[b]{2}{*}{$D, \mu$} \\
\hline & & & $\begin{array}{l}\text { Count Rate, } \\
\text { Counts/min }\end{array}$ & $\begin{array}{l}\text { Fraction of } \\
\text { Initial Rate, } \\
\text { per cent }\end{array}$ & \\
\hline $\begin{array}{l}0.5 \\
1.0 \\
1.5 \\
2.0 \\
2.5 \\
3.0 \\
3.5 \\
4.0 \\
4.5 \\
5 \cdot 0 \\
5.5 \\
6.0 \\
6.6 \\
7.0 \\
7 \cdot 5 \\
8.0 \\
8.5 \\
9.0 \\
9.5 \\
10.0 \\
11.5 \\
12 \\
13 \\
14 \\
15 \\
16 \\
17 \\
18 \\
19 \\
20 \\
24 \\
30 \\
35 \\
45 \\
67 \\
91 \\
122 \\
100 \\
4\end{array}$ & $\begin{array}{r}3,250 \\
6,450 \\
9,550 \\
12,550 \\
15,300 \\
18,000 \\
20,440 \\
22,700 \\
24,880 \\
26,780 \\
28,370 \\
29,800 \\
31,200 \\
32,100 \\
32,970 \\
33,820 \\
34,580 \\
35,280 \\
35,950 \\
36,570 \\
38,420 \\
38,980 \\
40,050 \\
41,120 \\
42,160 \\
43,140 \\
44,110 \\
45,100 \\
46,060 \\
46,980 \\
50,540 \\
55,160 \\
59,820 \\
67,830 \\
85,010 \\
103,010 \\
125,750 \\
125,750\end{array}$ & $\begin{array}{r}6,300 \\
5,700 \\
5,400 \\
4,880 \\
4,520 \\
4,360 \\
3,800 \\
3,180 \\
2,860 \\
2,330 \\
2,250 \\
1,740 \\
1,700 \\
1,520 \\
1,400 \\
1,340 \\
1,240 \\
1,170 \\
1,120 \\
1,070 \\
1,070 \\
1,040 \\
980 \\
990 \\
990 \\
960 \\
990 \\
800 \\
890 \\
850 \\
830 \\
800 \\
1780 \\
750 \\
730 \\
700\end{array}$ & $\begin{array}{r}5,600 \\
5,000 \\
4,700 \\
4,180 \\
3,820 \\
3,660 \\
3,100 \\
2,480 \\
2,160 \\
1,630 \\
1,550 \\
1,040 \\
1,000 \\
820 \\
700 \\
640 \\
540 \\
470 \\
420 \\
370 \\
370 \\
340 \\
280 \\
270 \\
290 \\
260 \\
220 \\
190 \\
150 \\
130 \\
100 \\
80 \\
50 \\
30 \\
0\end{array}$ & $\begin{array}{l}100 \\
89 \\
84 \\
75 \\
68 \\
65 \\
55 \\
44 \\
39 \\
29 \\
28 \\
19 \\
18 \\
15 \\
12.5 \\
11.5 \\
9.5 \\
8.5 \\
7.5 \\
6.5 \\
6.5 \\
6 \\
5 \\
5 \\
5 \\
4.5 \\
4 \\
3.5 \\
2.5 \\
2 \\
2 \\
1.5 \\
1 \\
0.5 \\
0\end{array}$ & $\begin{array}{l}28.7 \\
25.7 \\
23.4 \\
21.7 \\
20.3 \\
19.2 \\
18.2 \\
17.3 \\
16.6 \\
15.8 \\
15.3 \\
14.8 \\
14.4 \\
13.9 \\
13.5 \\
13.2 \\
12.8 \\
12.0 \\
11.7 \\
11.3 \\
10.8 \\
10.5 \\
10.1 \\
9.8 \\
9.6 \\
9.3 \\
9.1 \\
8.3 \\
7.4 \\
6.9 \\
6.0 \\
5.0 \\
4.0 \\
3.7 \\
1.2\end{array}$ \\
\hline
\end{tabular}


TABLE 17 (contd.)

RUN 14B

\begin{tabular}{|c|c|c|c|c|c|}
\hline \multirow[b]{2}{*}{$\begin{array}{l}\text { Time, } \\
\text { min. }\end{array}$} & \multirow[b]{2}{*}{ Counts } & \multirow[b]{2}{*}{$\begin{array}{l}\text { Count Rate, } \\
\text { Count } 8 / \mathrm{min}\end{array}$} & \multicolumn{2}{|c|}{ Corrected for Background } & \multirow[b]{2}{*}{$D, \mu$} \\
\hline & & & $\begin{array}{l}\text { Count Rate, } \\
\text { Count s/min }\end{array}$ & $\begin{array}{l}\text { Fraction of } \\
\text { Initial Rate, } \\
\text { per cent }\end{array}$ & \\
\hline $\begin{array}{l}0.5 \\
1.0 \\
1.5 \\
2.0 \\
2.5 \\
3.0 \\
3.5 \\
4.0 \\
4.5 \\
5.0 \\
5.5 \\
6.0 \\
6.5 \\
7.0 \\
7.5 \\
8.0 \\
8.5 \\
9.0 \\
9.5 \\
10.0 \\
10.5 \\
11 \\
12 \\
13 \\
14 \\
15 \\
16 \\
16 \\
20 \\
27 \\
54\end{array}$ & $\begin{array}{r}3,600 \\
7,000 \\
10,400 \\
13,800 \\
17,100 \\
20,300 \\
23,400 \\
26,370 \\
29,070 \\
31,370 \\
33,520 \\
35,280 \\
36,730 \\
37,950 \\
39,000 \\
39,920 \\
40,800 \\
41,610 \\
42,380 \\
43,060 \\
43,740 \\
44,340 \\
45,580 \\
46,690 \\
47,800 \\
48,890 \\
49,950 \\
53,880 \\
60,220 \\
82,710\end{array}$ & $\begin{array}{r}6,800 \\
6,600 \\
6,400 \\
6,200 \\
5,940 \\
5,400 \\
4,600 \\
4,300 \\
3,320 \\
2,900 \\
2,440 \\
2,100 \\
1,840 \\
1,760 \\
1,620 \\
1,540 \\
1,360 \\
1,360 \\
1,200 \\
1,240 \\
1,110 \\
1,110 \\
1,090 \\
1,060 \\
980 \\
910 \\
830\end{array}$ & $\begin{array}{r}6,100 \\
5,900 \\
5,700 \\
5,500 \\
5,240 \\
4,700 \\
3,900 \\
3,600 \\
2,620 \\
2,200 \\
1,740 \\
1,400 \\
1,140 \\
1,060 \\
920 \\
840 \\
660 \\
660 \\
500 \\
540 \\
410 \\
410 \\
390 \\
360 \\
280 \\
210 \\
130\end{array}$ & $\begin{array}{c}100 \\
97 \\
94 \\
90 \\
86 \\
77 \\
64 \\
59 \\
43 \\
36 \\
28 \\
23 \\
19 \\
17 \\
15 \\
14 \\
11 \\
11 \\
8 \\
9 \\
7 \\
7 \\
6 \\
6 \\
5 \\
3.5 \\
2\end{array}$ & $\begin{array}{r}28.7 \\
25.7 \\
23.4 \\
21.7 \\
20.3 \\
19.2 \\
18.2 \\
17.3 \\
16.6 \\
15.9 \\
15.3 \\
14.8 \\
14.4 \\
13.9 \\
13.5 \\
13.2 \\
12.8 \\
12.5 \\
12.2 \\
11.7 \\
11.3 \\
10.8 \\
10.5 \\
10.1 \\
9.1 \\
8.0 \\
5.5\end{array}$ \\
\hline
\end{tabular}


TABLE 17 (contd.)

RUN 16-19A

\begin{tabular}{|c|c|c|c|c|c|}
\hline \multirow[b]{2}{*}{$\begin{array}{r}\text { Time, } \\
\text { min. }\end{array}$} & \multirow[b]{2}{*}{ Counts } & \multirow[b]{2}{*}{$\begin{array}{l}\text { Count Rate, } \\
\text { Counts/min }\end{array}$} & \multicolumn{2}{|c|}{ Corrected for Background } & \multirow[b]{2}{*}{$D, \mu$} \\
\hline & & & $\begin{array}{l}\text { Count Rate, } \\
\text { Counts/min }\end{array}$ & $\begin{array}{l}\text { Fraction of } \\
\text { Initial Rate, } \\
\text { per cent }\end{array}$ & \\
\hline $\begin{array}{l}0.5 \\
1.0 \\
1.5 \\
2.0 \\
2.5 \\
3.0 \\
3.5 \\
4.0 \\
4.5 \\
5.0 \\
5.5 \\
6.0 \\
6.5 \\
7.0 \\
7.5 \\
8.0 \\
8.5 \\
9.0 \\
10 \\
11 \\
12 \\
15 \\
20 \\
30 \\
40 \\
60\end{array}$ & $\begin{array}{r}2,950 \\
5,950 \\
8,970 \\
11,900 \\
14,820 \\
17,700 \\
20,300 \\
22,550 \\
24,550 \\
26,230 \\
27,510 \\
28,540 \\
29,440 \\
30,180 \\
30,820 \\
31,380 \\
31,870 \\
32,380 \\
33,240 \\
34,070 \\
34,870 \\
37,210 \\
41,070 \\
48,310 \\
55,310 \\
69,290\end{array}$ & $\begin{array}{r}5,980 \\
5,860 \\
5,840 \\
5,760 \\
5,200 \\
4,500 \\
4,000 \\
3,360 \\
2,560 \\
2,060 \\
1,800 \\
1,480 \\
1,280 \\
1,120 \\
980 \\
1,020 \\
860 \\
830 \\
800 \\
780 \\
770 \\
725 \\
700 \\
700\end{array}$ & $\begin{array}{r}5,280 \\
5,160 \\
5,140 \\
5,060 \\
4,500 \\
3,800 \\
3,300 \\
2,760 \\
1,860 \\
1,360 \\
1,100 \\
780 \\
580 \\
420 \\
280 \\
320 \\
160 \\
130 \\
100 \\
80 \\
70 \\
25 \\
0 \\
0\end{array}$ & $\begin{array}{c}100 \\
98 \\
97 \\
96 \\
85 \\
72 \\
63 \\
52 \\
35 \\
26 \\
21 \\
15 \\
11 \\
8 \\
5 \\
6 \\
3 \\
2.5 \\
2 \\
1.5 \\
1.5 \\
0.5 \\
0 \\
0\end{array}$ & $\begin{array}{r}14.8 \\
12.8 \\
11.45 \\
10.45 \\
9.67 \\
9.05 \\
8.52 \\
8.10 \\
7.72 \\
7.39 \\
7.10 \\
6.84 \\
6.62 \\
6.40 \\
6.21 \\
6.03 \\
5.72 \\
5.45 \\
5.22 \\
4.67 \\
4.04 \\
3.30 \\
2.86 \\
2.33\end{array}$ \\
\hline
\end{tabular}


TABLE 17 (conta.)

RUN 16-19B

\begin{tabular}{|c|c|c|c|c|c|}
\hline \multirow[b]{2}{*}{$\begin{array}{l}\text { Time, } \\
\text { min. }\end{array}$} & \multirow[b]{2}{*}{ Counts } & \multirow[b]{2}{*}{$\begin{array}{l}\text { Count Rate, } \\
\text { Counts/min }\end{array}$} & \multicolumn{2}{|c|}{ Corrected for Background } & \multirow[b]{2}{*}{$D, \mu$} \\
\hline & & & $\begin{array}{l}\text { Count Rate, } \\
\text { Counts/min }\end{array}$ & $\begin{array}{l}\text { Fraction of } \\
\text { Initial Rate, } \\
\text { per cent }\end{array}$ & \\
\hline $\begin{array}{l}0.5 \\
1.0 \\
1.5 \\
2.0 \\
2.5 \\
3.0 \\
3.5 \\
4.0 \\
4.5 \\
5.0 \\
5.5 \\
6.0 \\
6.5 \\
7.0 \\
7.5 \\
8.0 \\
8.5 \\
9.0 \\
10 \\
11 . \\
12 \\
13 \\
14 \\
15 \\
20 \\
40 \\
95 \\
30\end{array}$ & $\begin{array}{r}2,900 \\
5,750 \\
8,750 \\
11,650 \\
14,400 \\
17,100 \\
19,500 \\
21,850 \\
23,760 \\
25.340 \\
26,500 \\
27,510 \\
28,320 \\
28,950 \\
29,600 \\
30,100 \\
30,580 \\
31,040 \\
31,920 \\
32,770 \\
33,570 \\
34,380 \\
35,090 \\
35,830 \\
39.510 \\
54,100 \\
93,110\end{array}$ & $\begin{array}{r}5,820 \\
5,500 \\
5,400 \\
4,800 \\
4,700 \\
3,820 \\
3,160 \\
2,320 \\
2,020 \\
1,620 \\
1,260 \\
1,300 \\
1,000 \\
960 \\
920 \\
880 \\
850 \\
800 \\
810 \\
710 \\
740 \\
736 \\
730 \\
710 \\
680\end{array}$ & $\begin{array}{r}5,140 \\
4,820 \\
4,720 \\
4,120 \\
4,020 \\
3,140 \\
2,480 \\
1,640 \\
1.340 \\
940 \\
580 \\
620 \\
320 \\
280 \\
240 \\
200 \\
170 \\
120 \\
130 \\
30 \\
60 \\
56 \\
50 \\
30 \\
0\end{array}$ & $\begin{array}{l}100 \\
94 \\
92 \\
80 \\
78 \\
61 \\
48 \\
32 \\
26 \\
18 \\
11 \\
12 \\
6 \\
5.5 \\
4.5 \\
4 \\
3 \\
2 \\
2.5 \\
0.5 \\
1 \\
1 \\
1 \\
0.5 \\
0\end{array}$ & $\begin{array}{l}12.5 \\
11.2 \\
10.2 \\
9.45 \\
8.83 \\
8.43 \\
7.90 \\
7.53 \\
7.21 \\
6.93 \\
6.68 \\
6.45 \\
6.24 \\
6.06 \\
5.78 \\
5.58 \\
5.33 \\
5.10 \\
4.90 \\
4.72 \\
4.56 \\
3.95 \\
2.79 \\
1.81 \\
0.55\end{array}$ \\
\hline
\end{tabular}


TABLE 17 (contd.) RUN 17-20A

\begin{tabular}{|c|c|c|c|c|c|}
\hline \multirow[b]{2}{*}{$\begin{array}{l}\text { Time, } \\
\text { min. }\end{array}$} & \multirow[b]{2}{*}{ Counts } & \multirow[b]{2}{*}{$\begin{array}{l}\text { Count Rate, } \\
\text { Counts/min }\end{array}$} & \multicolumn{2}{|c|}{ Corrected for Background } & \multirow[b]{2}{*}{$D, \mu$} \\
\hline & & & $\begin{array}{l}\text { Count Rate, } \\
\text { Counts/min }\end{array}$ & $\begin{array}{l}\text { Fraction of } \\
\text { Initial Rate, } \\
\text { per cent }\end{array}$ & \\
\hline $\begin{array}{l}1 \\
2 \\
3 \\
4 \\
5 \\
6 \\
7 \\
8 \\
9 \\
10 \\
11 \\
12 \\
13 \\
14 \\
16 \\
18 \\
20 \\
25 \\
31 \\
44 \\
66\end{array}$ & $\begin{array}{r}8,000 \\
15,950 \\
23,550 \\
30,900 \\
37,700 \\
43,650 \\
48,680 \\
52,430 \\
55,200 \\
57,290 \\
58,920 \\
60,330 \\
61,560 \\
62,740 \\
64,830 \\
66,750 \\
68,600 \\
72,860 \\
77,620 \\
87,500 \\
104,090\end{array}$ & $\begin{array}{r}7,970 \\
7,600 \\
7,350 \\
6,800 \\
5,950 \\
5,030 \\
3,750 \\
2,770 \\
2,090 \\
1,630 \\
1,410 \\
1,230 \\
1,180 \\
1,050 \\
960 \\
930 \\
850 \\
740 \\
760 \\
750\end{array}$ & $\begin{array}{r}7,250 \\
6,880 \\
6,630 \\
6,080 \\
5,230 \\
4,310 \\
3,030 \\
2,050 \\
1,370 \\
910 \\
690 \\
510 \\
460 \\
330 \\
240 \\
210 \\
130 \\
20 \\
40 \\
30\end{array}$ & $\begin{array}{c}100 \\
94 \\
92 \\
84 \\
72 \\
59 \\
42 \\
28 \\
19 \\
12.5 \\
9.5 \\
7 \\
6 \\
4.5 \\
3 \\
3 \\
2 \\
0.3 \\
0.5 \\
0.4\end{array}$ & $\begin{array}{l}12.5 \\
10.2 \\
8.8 \\
7.9 \\
7.2 \\
6.7 \\
6.25 \\
5.9 \\
5.6 \\
5.3 \\
5.1 \\
4.9 \\
4.7 \\
4.4 \\
4.15 \\
3.95 \\
3.5 \\
3.2 \\
2.65 \\
2.2\end{array}$ \\
\hline
\end{tabular}


TABLE 17 (contd.)

RUN 17-2OB

\begin{tabular}{|c|c|c|c|c|c|}
\hline \multirow[b]{2}{*}{$\begin{array}{c}\text { Time, } \\
\text { min. }\end{array}$} & \multirow[b]{2}{*}{ Counts } & \multirow[b]{2}{*}{$\begin{array}{l}\text { Count Rate, } \\
\text { Counts/min }\end{array}$} & \multicolumn{2}{|c|}{ Corrected for Background } & \multirow[b]{2}{*}{$D, \mu$} \\
\hline & & & $\begin{array}{l}\text { Count Rate, } \\
\text { Counts/min }\end{array}$ & $\begin{array}{l}\text { Fraction of } \\
\text { Initial Rate, } \\
\text { per cent }\end{array}$ & \\
\hline $\begin{array}{r}1 \\
2 \\
3 \\
4 \\
5 \\
6 \\
7 \\
8 \\
9 \\
10 \\
11 \\
12 \\
13 \\
14 \\
15 \\
17 \\
20 \\
23 \\
33 \\
38 \\
40 \\
42 \\
46 \\
49 \\
67 \\
88 \\
135 \\
167 \\
1,150 \\
2500\end{array}$ & $\begin{array}{r}7,300 \\
15,000 \\
22,000 \\
29,250 \\
35,660 \\
41,160 \\
45,680 \\
49,000 \\
51,500 \\
53,230 \\
54,710 \\
56,010 \\
57,300 \\
58,510 \\
59,600 \\
61,590 \\
64,470 \\
67,100 \\
76,140 \\
80,820 \\
82.440 \\
83.980 \\
87,010 \\
89,280 \\
102,800 \\
118,500 \\
153,150 \\
177,250\end{array}$ & $\begin{array}{r}7,500 \\
7,200 \\
7,050 \\
6,410 \\
5,500 \\
4,520 \\
3,320 \\
2,500 \\
1.730 \\
1,480 \\
1,300 \\
1,290 \\
1,210 \\
1,090 \\
1,000 \\
960 \\
880 \\
900 \\
940 \\
810 \\
770 \\
760 \\
760 \\
750 \\
750 \\
740 \\
750 \\
720 \\
715\end{array}$ & $\begin{array}{r}6,780 \\
6,480 \\
6,320 \\
5,690 \\
4,780 \\
3,800 \\
2,600 \\
1,780 \\
1,010 \\
760 \\
580 \\
570 \\
490 \\
370 \\
280 \\
240 \\
160 \\
180 \\
220 \\
90 \\
50 \\
40 \\
40 \\
30 \\
30 \\
20 \\
30 \\
0 \\
0\end{array}$ & $\begin{array}{l}100 \\
96 \\
93 \\
84 \\
71 \\
56 \\
38 \\
26 \\
15 \\
11 \\
8.5 \\
8.5 \\
7 \\
5.5 \\
4 \\
3.5 \\
2.5 \\
2.5 \\
3 \\
1.3 \\
0.7 \\
0.6 \\
0.6 \\
0.5 \\
0.5 \\
0.3 \\
0.5 \\
0 \\
0 \\
0\end{array}$ & $\begin{array}{l}12.5 \\
10.2 \\
8.8 \\
7.9 \\
7.2 \\
6.7 \\
6.25 \\
5.9 \\
5.6 \\
5.3 \\
5.1 \\
4.9 \\
4.7 \\
4.55 \\
4.3 \\
3.95 \\
3.7 \\
3.1 \\
2.85 \\
2.8 \\
2.7 \\
2.6 \\
2.5 \\
2.15 \\
1.9 \\
1.5 \\
1.4 \\
0.5 \\
0.35\end{array}$ \\
\hline
\end{tabular}


Subtraction of the background from this count rate gave the count rate due to the particles passing the window. The fraction of the initial count rate is equivalent to the mass fraction of particles still in the counting zone. The last column gives the diameter of the particles which should have falled out of the counting zone, according to Stoke's law. 
APPENDIX II - Derivations 


\section{DERIVATIONS}

Range-energy relationsh1p

From Toblas (44)

$$
\frac{N(p)}{N} d p=\frac{3}{2 D}\left[1-\left(\frac{p}{D}\right)^{2}\right] d p,
$$

where $p$ is the path length of a fragment inside the sphere, and $\mathrm{N}(\mathrm{p}) / \mathrm{N}$ is the fraction of fragments with that path length. Then,

$$
\frac{E(p)_{d p}}{E_{0}}=\frac{3}{2 D}\left[1-\left(\frac{p}{D}\right)^{2}\right]\left[1-\frac{p}{R}\right]^{3 / 2} d p,
$$

where $E(p) / E_{0}$ is the fractional energy of the particle with path length $p$. To obtain the total fraction of energy that escapes, equation (26) is integrated over the limit of $p$, which, for $0 \leq R \leq D$, are $O$ to $R$.

$$
\frac{\bar{E}}{E_{0}}=\frac{3}{2 D} \int_{0}^{R}\left[1-\frac{p}{R}\right]^{3 / 2}\left[1-\left(\frac{p}{D}\right)^{2}\right] d p
$$

Let $u=1-p / R$, then $d u=-d p / R$. Substituting,

$$
\begin{aligned}
\frac{\bar{E}}{E_{0}} & =-\frac{3}{2 D} \int_{I}^{R}\left[I-\frac{R^{2}}{D^{2}}(1-u)^{2}\right] u^{3 / 2} R d u \\
& =\frac{3}{5} \frac{R}{D}-\frac{8}{105} \frac{R^{3}}{D^{3}}, 0 \leq R \leq D
\end{aligned}
$$

For $\mathrm{R}>\mathrm{D}$, integrate between 1 and $1-\mathrm{D} / \mathrm{R}$ :

$$
\begin{gathered}
\frac{\bar{E}}{E_{0}}=\left[\frac{3 R}{5 D}-\frac{8 R^{3}}{105 D^{3}}\right]-\frac{3 R}{5 D}\left(1-\frac{D}{R}\right)^{5 / 2}+\frac{3 R^{3}}{5 D^{3}}\left(1-\frac{D}{R}\right)^{5 / 2} \\
-\frac{6 R^{3}}{7 D^{3}}\left(1-\frac{D}{R}\right)^{7 / 2}+\frac{R^{3}}{3 D^{3}}\left(1-\frac{D}{R}\right)^{9 / 2}
\end{gathered}
$$


APPENDIX III - Sample Calculations 


\section{SAMPLE CALCULATIONS}

\section{Range}

The relationship of the range of the light fission fragment in oxygen to that in aluminum is as follows:

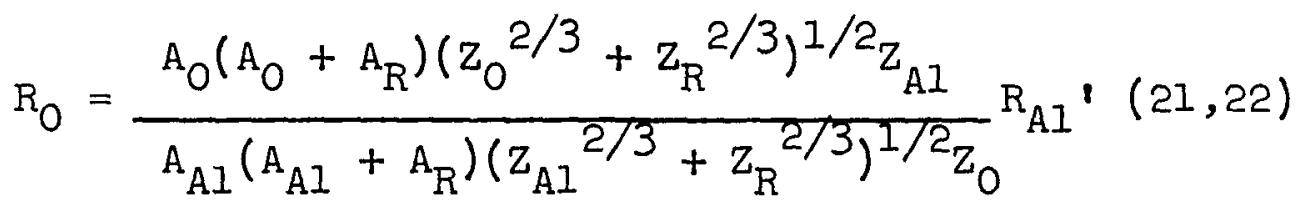

$$
\begin{aligned}
& R_{0}^{\prime}=\frac{16(16+95)\left(8^{2 / 3}+40^{2 / 3}\right)^{1 / 2}(13)}{27(27+95)\left(13^{2 / 3}+40^{2 / 3}\right)^{1 / 2}(8)} R_{A 1}^{\prime} \\
& \mathrm{R}_{\mathrm{O}}{ }^{\prime}=0.835 \mathrm{R}_{\mathrm{Al}}{ }^{\prime} \mathrm{mg} / \mathrm{cm}^{2}
\end{aligned}
$$
follows:

$$
\begin{aligned}
& \frac{1}{\mathrm{R}^{\prime}{ }_{10 \% \mathrm{UO}_{2}-\mathrm{ThO}_{2}}}=\frac{0.1}{\mathrm{R}_{\mathrm{UO} \mathrm{O}_{2}}}+\frac{0.9}{\mathrm{R}^{\prime} \mathrm{ThO}_{2}} \\
& =\frac{0.1}{8.84}+\frac{0.9}{8.58} \\
& \mathrm{R}^{\mathrm{R}}{ }_{10 \% \mathrm{UO}_{2}}-\mathrm{ThO}_{2}=8.60 \mathrm{mg} / \mathrm{cm}^{2}
\end{aligned}
$$

Range-energy relationship

In Figure 1 for ratios of the diameter to range greater than one, equation (29) was used for the zero concentration curve. For $\mathrm{D} / \mathrm{R}_{\mathrm{S}}=2.0$,

$$
\frac{\bar{E}}{\bar{E}_{O}}=\frac{3}{5}\left(\frac{1}{2}\right)-\frac{8}{105}\left(\frac{1}{8}\right)=0.29
$$


For ratios of the diameter to range less than one, equation (24) was used. For the zero concentration curve and for $D / R_{S}=0.5$,

$$
\frac{\overline{\mathrm{E}}}{\overline{\mathrm{E}}_{\mathrm{O}}}=i-0.48(0.5)=0.76
$$

$$
\begin{aligned}
& \text { For } C / p=0.10, R_{f} / R_{S}=3.0 \text {, and } D / R_{S}=0.5, \\
& \begin{aligned}
\frac{\bar{E}}{E_{0}}=\frac{1}{1+\frac{0.1}{1-0.1}(3)} & -0.48\left[e^{-1.8(0.1)(3)] 0.5}\right. \\
= & 0.61
\end{aligned}
\end{aligned}
$$

Gas analysis

Run 20, sample 9:

$$
\begin{aligned}
\text { Hydrogen content }=\frac{43.7}{2} & \mathrm{~cm}^{2} \frac{\left(0.0423 \mathrm{~mm} \mathrm{H}^{2} / \mathrm{cm}^{2}\right)}{134 \mathrm{~mm}^{2}} \\
& =0.690 \% \mathrm{H}_{2}
\end{aligned}
$$

Fission product analysis

$$
\begin{aligned}
\text { Run 17: Total number of fissions } & \\
= & 62.7 \times 10^{10} \mathrm{f} / \mathrm{gm} \mathrm{H}_{2} \mathrm{O}\left(8.5 \mathrm{gm} \cdot \mathrm{H}_{2} \mathrm{O}\right) \\
& +54 \times 10^{10} \mathrm{f} / \mathrm{gm} \mathrm{s} .(3.165 \mathrm{gm} . \mathrm{s} .) \\
= & 741 \times 10^{10} \text { fissions }
\end{aligned}
$$

$\mathrm{Cr}^{51}$ Monttor

Run 17: Between the limits of $240 \mathrm{kev}$, and $390 \mathrm{kev} .$, 38,793 counts were recorded in one minute. At the time the count was recorded, the activity had decayed by the factor,

$$
\begin{aligned}
& -\frac{0.693 \text { (9.83 days) }}{27.8 \text { days }} \\
& e^{27.8 \text { days }}=0.782
\end{aligned}
$$




$$
\underline{x}
$$




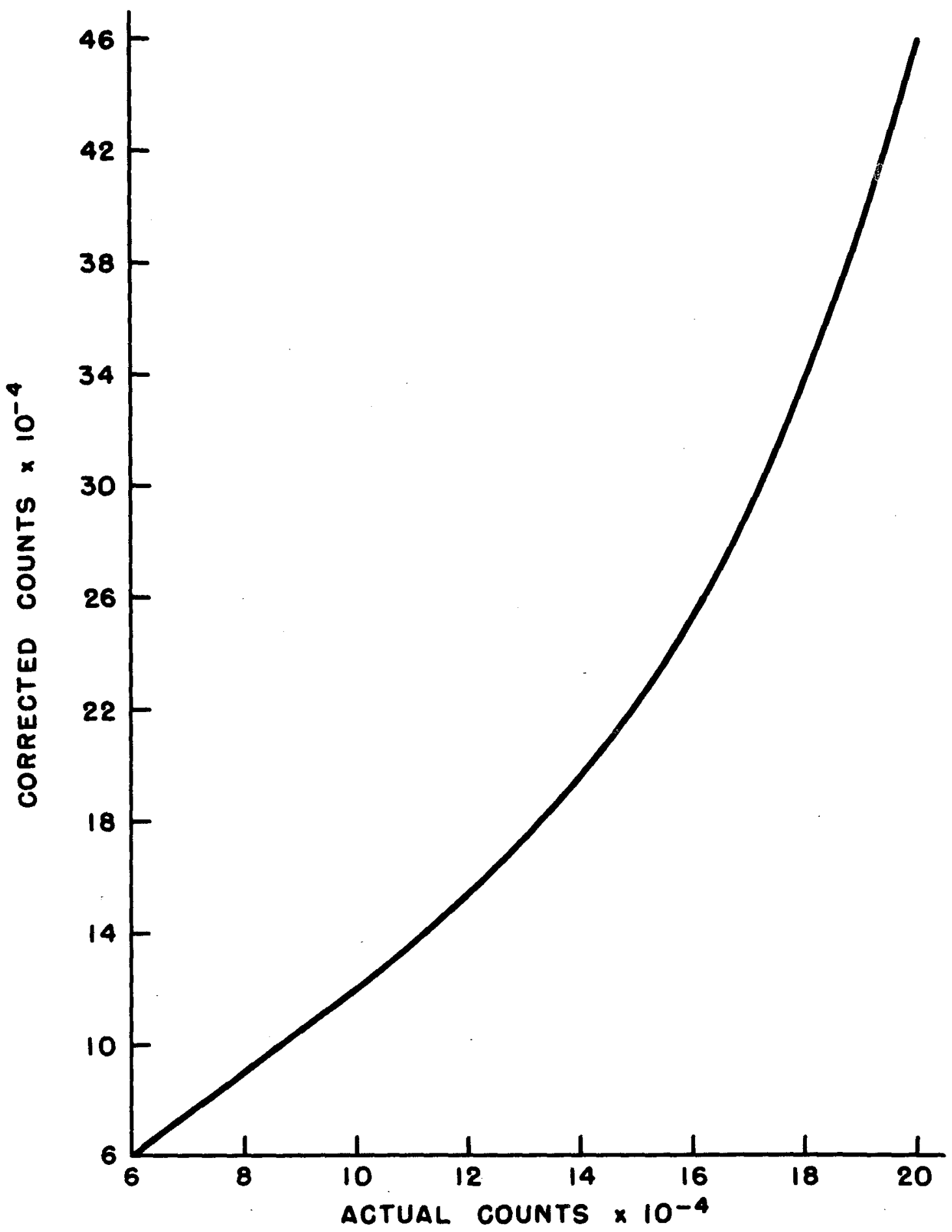

Figure 30. Calibration curve for fission product monitor. 
The relative flux then becomes:

$$
\frac{38,793 \text { counts }}{42.5 \mathrm{mg} .(24.3 \mathrm{~min} .)(0.782)}=47.9 \mathrm{c} / \mathrm{mg} / \mathrm{min}
$$

Gas flow rate

Run 17: The mean flow rate is calculated as follows:

$$
\begin{gathered}
\frac{1}{19} \sum_{1}^{19}\left(\frac{10}{\text { flow measurement, sec } / 10 \mathrm{cc}}\right)=0.305 \mathrm{cc} / \mathrm{sec}(44) \\
\overline{\mathrm{x}}_{1}=0.305 \mathrm{cc} / \mathrm{sec}\left(\frac{60 \mathrm{sec} .}{\mathrm{min} .}\right)\left(\frac{734.8-28.3}{760}\right)\left(\frac{273}{301}\right) \\
=15.4 \mathrm{cc} / \mathrm{min}, \mathrm{STP}
\end{gathered}
$$

\section{Energy determination}

Run 17:

Fission yield $=\frac{741 \times 10^{10} \text { fissions }}{3.165 \mathrm{gm} \cdot(0.0938 \mathrm{gm} \mathrm{U} / \mathrm{gm})(24.3 \mathrm{~min})}$

$$
=1.03 \times 10^{12} \mathrm{f} / \mathrm{gm} \mathrm{U} / \mathrm{min}
$$

Monitor factor $=\frac{1.03 \times 10^{12} \mathrm{f} / \mathrm{gm} \mathrm{U} / \mathrm{min}}{47.9 \mathrm{c} / \mathrm{mg} / \mathrm{min}}=2.15 \times 10^{10}$

Since the mean monitor factor is equal to $2.18 \times 10^{10}$,

Corrected yield $=1.03 \times 10^{12} \mathrm{f} / \mathrm{gm} \mathrm{U} / \mathrm{min}\left(\frac{2.18}{2.15}\right)$

$$
=1.10 \times 10^{12}
$$

Total energy $=1.10 \times 10^{12} \mathrm{f} / \mathrm{gm} \mathrm{U} / \mathrm{min}$

$(3.165 \mathrm{gm})(0.0938 \mathrm{gm} \mathrm{U} / \mathrm{gm}) 165 \mathrm{Mev} / \mathrm{f})$

$8.5 \mathrm{gm} \cdot \mathrm{H}_{2} \mathrm{O}$

$=6.34 \times 10^{12} \mathrm{Mev} / \mathrm{min} / \mathrm{gm} \mathrm{H}_{2} \mathrm{O}$ 
The fraction of this energy which is effective was obtained by averaging the effective fractions of the light and heavy fragments as follows:

$$
\frac{100 \mathrm{Mev} \cdot(0.60)+65 \mathrm{Mev} \cdot(0.53)}{165 \mathrm{Mev} .}=0.572
$$

Particle size

Stoke's Law may be written:

$$
\mathrm{v}, \mathrm{cm} / \mathrm{sec},=\frac{1 D^{2}}{18} \frac{\left(\rho-P_{1}\right) \mathrm{g} \times 10^{-8}}{\eta}
$$

The values of the constants are $p=9.75 \mathrm{~g} / \mathrm{cc}$, $\rho_{1}=1.00 \mathrm{~g} / \mathrm{cc}, \eta=0.893 \times 10^{-2} \mathrm{p} ., \mathrm{g}=980.3 \mathrm{~cm} / \mathrm{sec}^{2}$, and $\mathrm{v}=10 \mathrm{~cm} / 606 \mathrm{sec}$.

Then $\quad D^{2}=\frac{3.125 \times 10^{2}}{t}$

and, $\quad D=\frac{17.65}{t^{1 / 2}}$ microns

The average diameter is obtained from the sedimentation rate data as follows:

$$
\sum_{n=1}^{\infty}\left(F_{n-1}-F_{n}\right)\left(D_{n}\right)=6.43 \mu
$$

where $\mathrm{n}$ is the number of measurements from zero time and $F_{n}$ is the fraction of the initial count rate at $n$. The energy-weighted diameter is calculated:

$$
\sum_{n=1}^{\infty}\left(F_{n-1}-F_{n}\right)\left[E\left(D_{n}\right)\right]=0.617,
$$

where $E\left(D_{n}\right)$ is the fraction of energy which is effective for a particle of diameter $D_{n}$ and is read from curve $A$ in 
Figure 1. The result, 0.617 , corresponds to a diameter of 6.31 microns.

\section{F1ssion product monitor}

Because of dead-t1me, the maximum count rate for the amplifier used in this experiment was $9.5 \times 10^{6}$ counts $/ \mathrm{min}$. Since the 3 per cent window width allowed only a fraction of the counts to appear on the scaler, the maximum count rate under these conditions was estimated to be 230,000 . On this basis the calibration curve in Figure 27 was calculated. 
APPENDIX IV - Computer Program 


\section{APPLIED MATHEMATICS DIVISION}

Program L1brary $1320 /$ CHM 145

May 9, 1962

TITLE: Monte Carlo Calculation of the Energy Lost in a Slurry System

PROGRAMMER: Daniel Carson

DESCRIPTION OF THE PROBLEM:

I. Physical Problem

The Slurry System consists of a solute dissolved in a solvent. The solute is such that it will or can be made to emit particles. The problem is then to determine what fraction of their initial energy the emitted particles will lose to the solvent.

The energy-loss relationship used in this problem is

$$
E_{x} / E_{0}=(1-x / R)^{3 / 2} \text {, }
$$

where $E_{x}$ is the energy of the emitted particle after it has traveled a distance $x$ in a medium where its maximum range is $R ; E_{0}$ is its initial energy.

II. Mathematical Approach

1. Slurry Generation

A system of $N$ non-intersecting spheres representing atoms of the dissolved solute is randomly generated within a cube. The cube edge is determined by the relationship

$$
d=D\left[\frac{N \cdot \pi / 6}{C / p}\right]^{1 / 3} \text {, }
$$

where

$$
d=\text { cube edge, }
$$


$D=$ diameter of the spheres representing the dissolved solute,

and $c / p=$ the rat1o of the total volume of the spheres to the total volume of the slurry. The criterion for the spheres to be non-intersecting is

(1) $D \leq\left|r_{1}-r_{j}\right|, 1=1, \ldots, N ; \quad j=1, \ldots, N$; where $r_{1}$ is the distance from the $1^{\text {th }}$ sphere center to the origin. If the spheres are to be within the cube, we must impose the constraints:

$$
\text { (2) } \begin{aligned}
r_{x 1} & \leq \frac{d-D}{2} \\
r_{y 1} & \leq \frac{d-D}{2} \\
r_{z 1} & \leq \frac{d-D}{2}
\end{aligned}
$$

The procedure is then to generate a random number $R$, $-1 \leq R \leq+1$, and multiply $R$ by $\left(\frac{d-D}{2}\right)$. The result is a coordinate of a sphere. When 3 coordinates have been chosen this way, we have satisfled (2.). In order to satisfy (1) we then check this point against all previous points. If the point cannot be accepted (1), three new coordinates are obtained and the new point checked.

\section{Sampling}

The spheres are numbered (e.g., sphere 1 has center $x_{1}, y_{1}, z_{1}$, etc.). A sphere number is then chosen at random. Since we are dealing with a slurry of finite size, only those spheres whose centers are within an inner volume are considered. This inner volume is such that a particle 
emitted from any point on a contained sphere will lack sufficient energy to leave the slurry.

The program deals with those particles emitted in the positive and negative $x$ direction. Therefore the inner volume used is a parallelepiped centered about the orlgin and whose dimensions are (see Flgure 31),

$$
\begin{aligned}
& \left|x_{\text {max }}\right|=\frac{d-3 D}{2}-R_{w} \\
& \left|z_{\text {max }}\right|=\left|Y_{\text {max }}\right|=\frac{d-3 D}{2} .
\end{aligned}
$$

Once a sphere within the inner volume has been found, a point on the sphere is randomly chosen and is used as the starting point for two histories.

\section{Histories}

A history consists of three steps:

a) Computing and recording the percent of energy lost by an emitted particle before it leaves the parent sphere. This statistic is entered Into a histogram whose intervals of percent of energy lost are 10\%.

b) Calculating a new range each time the particle enters a different medium using the relation$\operatorname{sh} 1 p$

$$
\text { New range }=\text { initial range } *\left(E_{x} / E_{0}\right)^{2 / 3} \text {, }
$$
where $\left(E_{x} / E_{0}\right)$ is the fraction of its initial energy the particle has at the interface of the two media. 


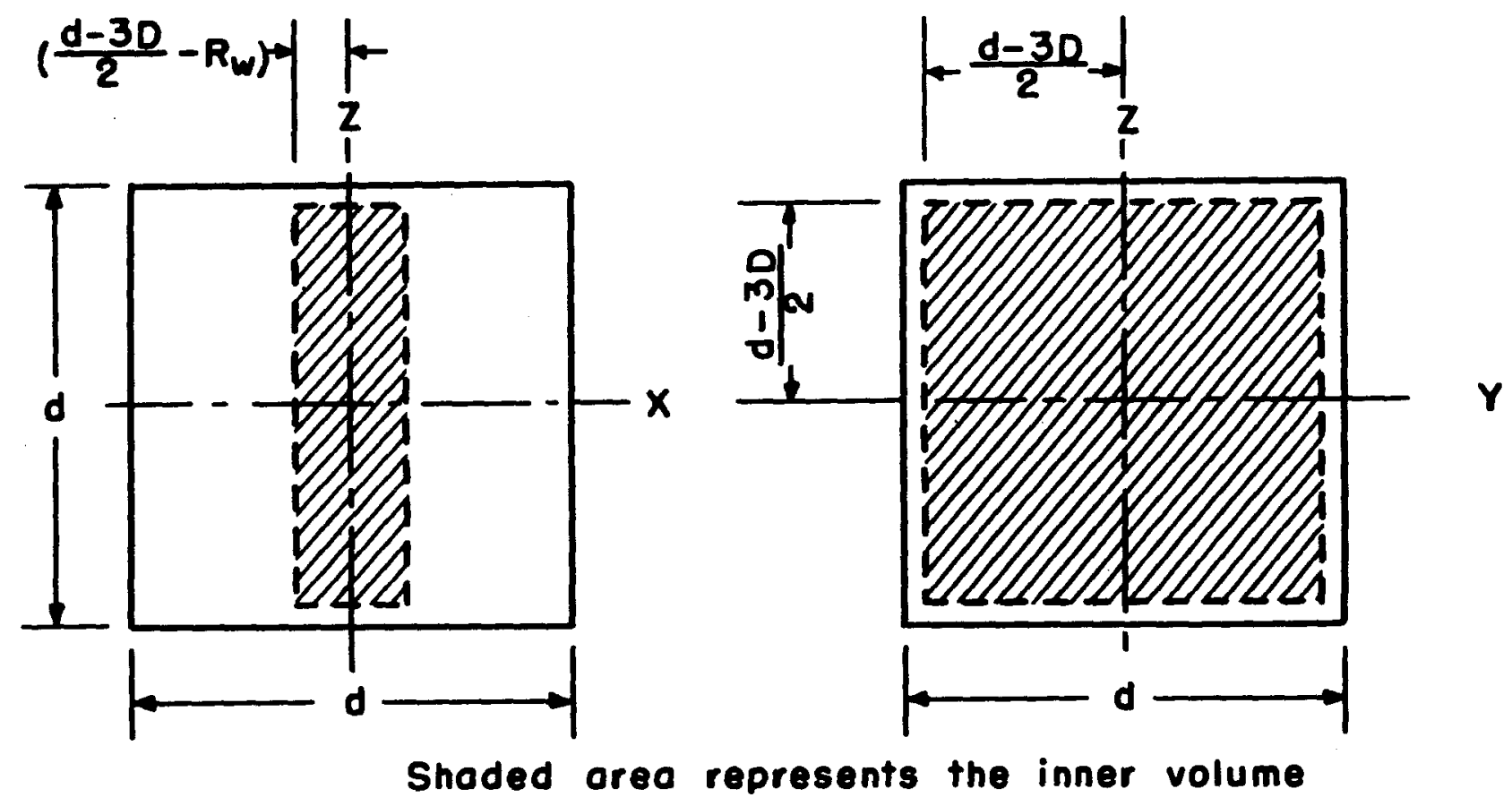

Figure 31. Volume of slurry considered in the computer progrom. 
c) Determining what fraction of its initial energy the emitted particle loses to the solvent before 1 t stops, and keeplng track of which medium stopped the particle.

A history in the positive $x$ direction is followed by one in the negative $x$ direction. After these two histories are taken for a particular point, a new sphere and point are randomly chosen and two further histories taken. This procedure is repeated until a block has been completed. After each block the average $\mathrm{E} / \mathrm{E}_{\mathrm{O}}$ lost to the fluid of all the preceding histories (1.e., the average for the block just completed plus all previous blocks) is printed. Thus the last $\mathrm{E} / \mathrm{E}_{\mathrm{O}}$ wlll be an average for (No. of blocks) * (No. of historles per block) histories. The standard deviation is also printed. The standard deviation is calculated by the relationship

Standard deviation $=\left[\frac{\sum_{i=1}\left(E / E_{0}(i)\right)^{2}}{H}-\left(\sum_{i=1}^{H} \frac{E / E_{0}(i)}{H}\right)^{2}\right]^{1 / 2}$ $\mathrm{E} / \mathrm{E}_{\mathrm{O}}(1)=$ average energy lost to fluid at $i^{\text {th }}$ history $H=$ total number of histories

MACHINE: $704 \mathrm{~F}$

RUNNING TIME: Some typical times for $R_{f} / R_{S}=3.0$ are 


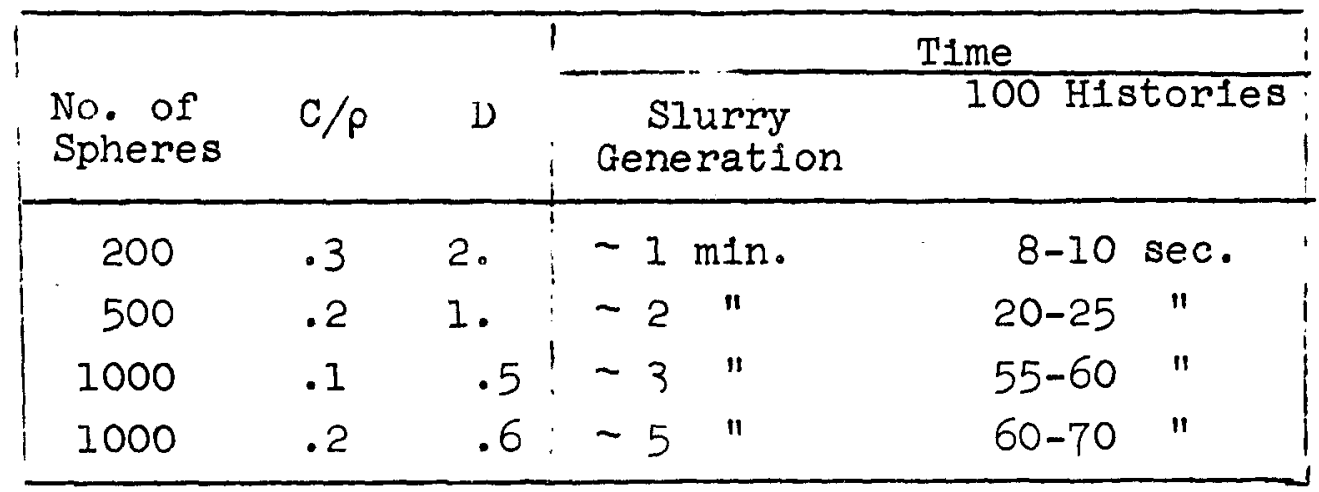

INPUT: The input for each case is on two cards. The first card contains five integers in six character fields. The integers are:

Ist interger $=$ number of spheres to be generated $(N)$

and interger $=$ number of attempts to fit $N$ spheres into the cube

$3 r d$ integer $=$ If $\mathrm{N}$ spheres cannot be fitted into the cube within the allowed number of tries, the slurry generation is started over. This interger defines the number of times the slurry generation is to be attempted

4 th integer $=$ number of histories per block

5 th integer $=$ number of blocks per case.

The second card contains three floating point decimal numbers in 12 character fields. The numbers are:

lst number $=\mathrm{C} / \mathrm{P}$

2nd number $=\mathrm{R}_{\mathrm{f}} / \mathrm{R}_{\mathrm{S}}$

$3 r d$ number $=D($ diameter of sphere $)$ 
OUTPUT: If the parameters are such that there is no inner volume, the parameters are printed out with an identifying heading. Machine will then pause $444448^{\circ}$ Pushing START w1Il cause next case to be read in.

If an inner volume is possible, the parameters are printed out and after each block of histories the average $\mathrm{E} / \mathrm{E}_{\mathrm{O}}$ lost to the solvent is printed out along with the standard deviation. (See Sample Output)

SPECIAL FEATURES:

1. If a case is run with sense switch 6 down; after Its completion, a new slurry is generated and and the same case repeated until switch is raised.

2. If $N$ spheres cannot be fitted into the cube in the specifled number of attempts, the spheres successfully fltted on the last attempt into the cube are used and a new $c / P$ is calculated. The problem then continues using the new values of $C / P$ and $N$.

3. The program cails the subroutine ANMIOI (ALGEBRAIC SORT) to sort the $x$ coordinates of the spheres. 
TABLE 18

SAMPLE OF DATA - MONTE CARLO CALCULATION OF ENERGY LOST IN $\mathrm{UO}_{2}-\mathrm{ThO}_{2}$ SLURRY

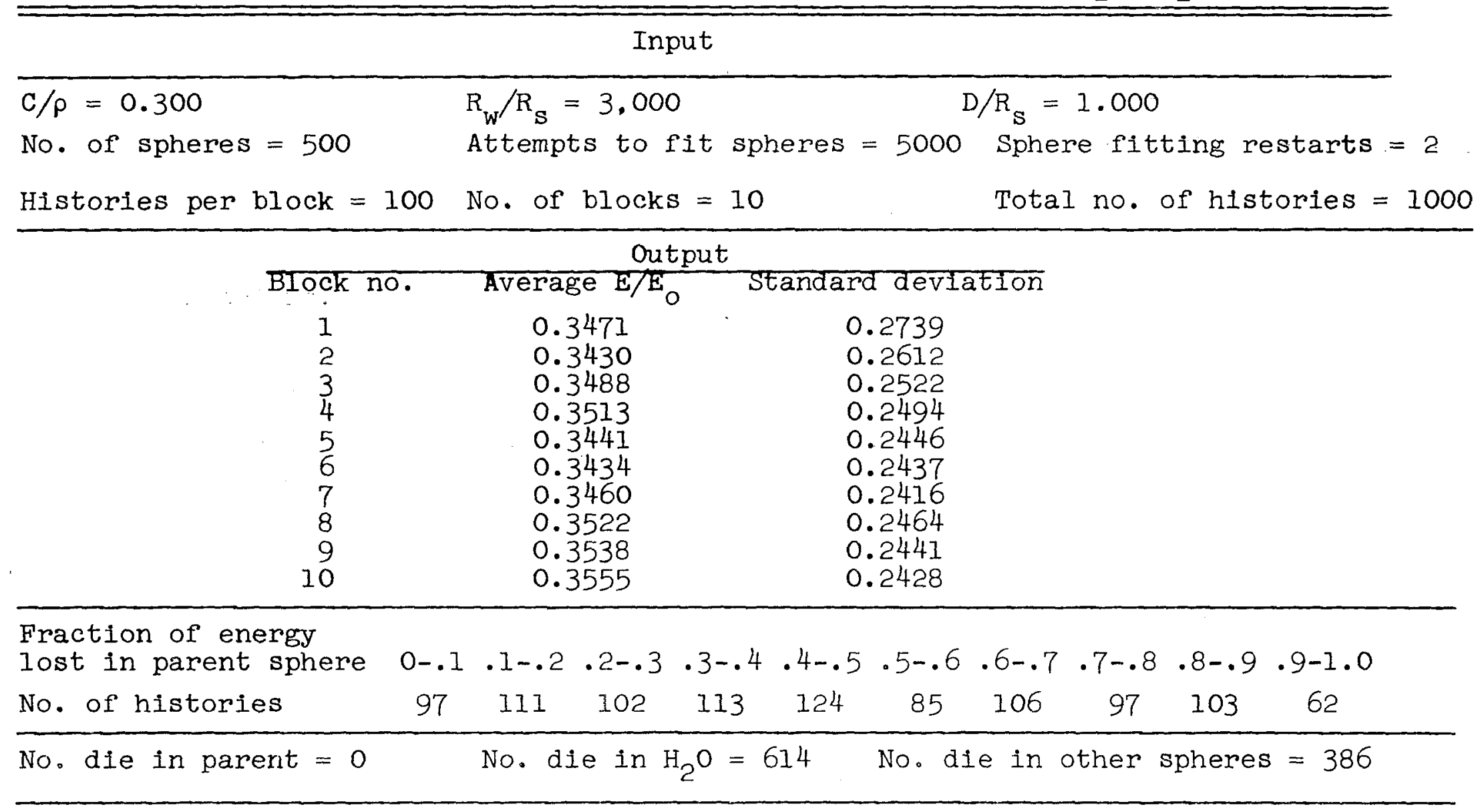




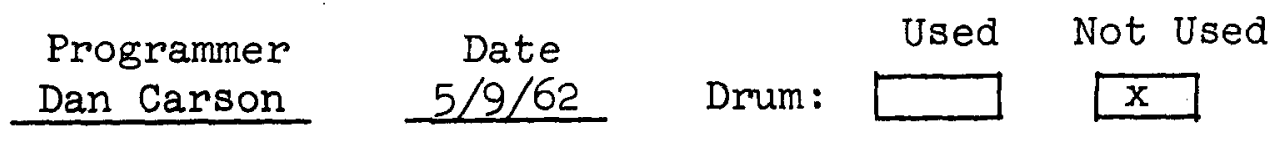

READER: Standard 72 column UF Switch: $\square$ PUNCH: not used

PRINTER: SHARE 2 board

Sense Switch Settings: all UP

TAPES: none

\section{Input:}

Scratch:

Output - Printed

To Be Saved

- Punched

Rewound by Program Prior to Calculation none After none Manual EOF Needed no

TIME BEFORE OUTPUT: 5-20 min. NORMAL RUNNING TIME: $30 \mathrm{~min}$. RUN NO LONGER THAN: $1-1 / 2$ hrs.

RUNNING PROCEDURE: (Indicate both regular and restart)

1. READY code deck and data deck in card reader.

2. CLEAR and LOAD CARDS.

3. When reader SELECT light is on and card reader is empty, problem is finished.

STOPS(OCTAL): 44444. Current case cannot be handled. To process next case, START the machine. 


\section{AUTOBIOGRAPHY}

I, Lawrence Russell Steele, was born in Manhattan, Kansas, November 7, 1935. I received my secondary education in Tecumseh High School, New Carlisle, Ohlo, from which I was graduated in 1953. By enrolling in both the college of Engineering and the Graduate School during my senior year at Oh1o State UnIversity, I was able to recelve both the Bachelor of Chemical Engineering and the Master of Science degrees in 1958. In September of that year I accepted an appointment to the International School of Nuclear Science and Englneering at Argonne National Laboratory, from which I recelved a certificate in June, 1959. I then returned to Ohio State University where I was appointed a Lubrizol Fellow in the Department of Chemical Engineering. Upon completion of the general requirements, I was appointed a Resident Student at Argonne National Laboratory in October, 1960, where I have completed the thesis requirements for the Doctor of Philosophy degree.

/hw 6/26/62. 